\title{
Modelling of Planetary Gravitational Microlensing Events
}

\author{
by \\ Michael L. J. Miller
}

A thesis submitted to Victoria University of Wellington in fulfilment of the requirements for the degree of Master of Science in Physics. 



\begin{abstract}
This thesis describes and develops procedures for the generation of theoretical lightcurves that can be used to model gravitational microlensing events that involve multiple lenses. Of particular interest are the cases involving a single lens star with one or more orbiting planets, as this has proven to be an effective way of detecting extrasolar planets. Although there is an analytical expression for microlensing lightcurves produced by single lensing body, the generation of model lightcurves for more than one lensing body requires the use of numerical techniques. The method developed here, known as the semi-analytic method, involves the analytical rearrangement of the relatively simple 'lens equation' to produce a high-order complex lens polynomial. Root-finding algorithms are then used to obtain the roots of this 'lens polynomial' in order to locate the positions of the images and calculate their magnifications.

By running example microlensing events through the root-finding algorithms, both the speed and accuracy of the Laguerre and Jenkins-Traub algorithms were investigated. It was discovered that, in order to correctly identify the image positions, a method involving solutions of several 'lens polynomials' corresponding to different coordinate origins needed to be invoked. Multipole and polygon approximations were also developed to include finite source and limb darkening effects. The semi-analytical method and the appropriate numerical techniques were incorporated into a $\mathrm{C}++$ modelling code at VUW (Victoria University of Wellington) known as mlens2. The effectiveness of the semi-analytic method was demonstrated using mlens2 to generate theoretical lightcurves for the microlensing events MOA-2009-BLG-319 and OGLE-2006-BLG-109. By comparing these theoretical lightcurves with the observed photometric data and the published models, it was demonstrated that the semi-analytic method described in this thesis is a robust and efficient method for discovering extrasolar planets.
\end{abstract}




\section{Contents}

1 Introduction to Gravitational Microlensing 1

2 Microlensing Theory $\quad 9$

2.1 Einstein Ring . . . . . . . . . . . . . . . . . . . . . 9

2.2 Lens Equation . . . . . . . . . . . . . . . . . . . . . 14

2.3 Complex Representation . . . . . . . . . . . . . . . . . 16

2.4 Multiple Lenses . . . . . . . . . . . . . . . . . . . . . . . . . 20

2.5 Semi-Analytical Method . . . . . . . . . . . . . . 23

2.6 Number of Images . . . . . . . . . . . . . . . . . . . . . 25

2.7 Orbital Motion . . . . . . . . . . . . . . . . . 27

3 Image Topology $\quad 29$

3.1 Point Source Magnification . . . . . . . . . . . . . . . . . 30

3.1.1 Single Lens Magnification . . . . . . . . . . . . . . 31

3.1.2 Multiple Lens Magnification . . . . . . . . . . . . . . 34

3.2 Image Topology . . . . . . . . . . . . . . . . . . . . . . 36

3.3 Critical and Caustic Curves . . . . . . . . . . . . . . . 40

3.4 Binary Lens . . . . . . . . . . . . . . . . . . . . . 42

3.4.1 Caustic and Critical Curves . . . . . . . . . . . . 42

3.4 .2 Point Source Magnification . . . . . . . . . . . . . 47

3.4.3 Image Positions and Magnifications . . . . . . . . . . . . . 49

3.4.4 Analysing Real Lightcurves . . . . . . . . . . . . . . 54

3.5 Higher Lens Configurations . . . . . . . . . . . . 56

3.5.1 Unphysical Root Pairs . . . . . . . . . . . . . 57

3.5.2 Multiple Planetary Events . . . . . . . . . . . . . . . . 60 
3.5.3 Additive Nature of Caustic and Critical Curves . . . . . . 61

3.5.4 Additive Nature of Planetary Lightcurves . . . . . . . . . . 62

$\begin{array}{lll}4 & \text { Finite Source Effects } & 67\end{array}$

4.1 Finite Source Disk _ . . . . . . . . . . . . . . . . . . . 68

4.2 Limb Darkening . . . . . . . . . . . . . . . . . . 71

4.3 Polygon Method . . . . . . . . . . . . . . . . . . . . . . 74

4.3.1 Area of a Polygon . . . . . . . . . . . . . . . . . . 74

4.3.2 Constructing Polygon . . . . . . . . . . . . . . . . 76

4.3.3 Limb Darkening . . . . . . . . . . . . . . . . 79

4.4 Multipole Approximations . . . . . . . . . . . . . . . . . . . . . 81

4.4.1 Derivation of Multipole Approximations . . . . . . . . 82

4.4.2 Single-Point Multipole Approximations . . . . . . . . . . . 84

4.4.3 Multiple-Point Multipole Approximations . . . . . . . . 85

4.5 Selection Algorithm . . . . . . . . . . . . . . . . . . . 91

5 Numerical Algorithms $\quad 95$

5.1 Laguerre Algorithm . . . . . . . . . . . . . . . . . . . 96

5.1 .1 Deflation ..................... 96

5.1 .2 Polishing . . . . . . . . . . . . . . . . . . . . 97

5.1.3 Breaking Limit Cycles . . . . . . . . . . . . . . . . . . 97

5.2 Jenkins-Traub Algorithm . . . . . . . . . . . . . . . . . 97

5.3 Algorithm Accuracy and Efficiency . . . . . . . . . . . . . . . 99

5.3.1 Accuracy and Efficiency of Numerical Techniques . . . . . 100

5.3 .2 Origin Shifting . . . . . . . . . . . . . . . . . . 104

6 Modelling Gravitational Microlensing Events 107

6.1 Modelling Procedure . . . . . . . . . . . . . . . 108

6.2 MOA-2009-BLG-319 . . . . . . . . . . . . . . 111

6.3 OGLE-2006-BLG-109 . . . . . . . . . . . . . . . 115 
A.1 Two Lens System - Degree 5 Lens Polynomial . . . . . . . . . . . 129

A.2 Three Lens System - Degree 10 Lens Polynomial . . . . . . . . . . 131

A.3 Solutions as the Source Position goes to Infinity . . . . . . . . . 135

$\begin{array}{ll}\text { B The Jacobian } & 137\end{array}$

B.1 Complex Coordinates . . . . . . . . . . . . . . . . 138

B.2 Derivative of the Jacobian . . . . . . . . . . . . . . 141

B.3 Derivative of the Magnification . . . . . . . . . . . . . . . . . 142

C Derivation of Critical Curve Polynomials 145

C.1 Two Lens System - Degree 4 Critical Curve Polynomial . . . . . . 148

C.2 Three Lens System - Degree 6 Critical Curve Polynomial . . . . . 149

$\begin{array}{ll}\text { D Quadrapole and Hexadecapole Approximations } & 151\end{array}$

D.1 Finite Source Disk . . . . . . . . . . . . . . . . . . 152

D.2 Taylor Expansion . . . . . . . . . . . . . . . . . 153

D.3 Polar Coordinates . . . . . . . . . . . . . . . . . . . . . 154

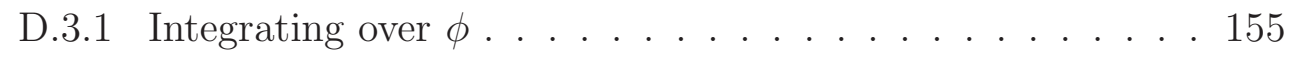

D.3.2 Integrating over $r \ldots \ldots \ldots \ldots \ldots$. . . . . . . . . 157

D.4 Limb Darkening . . . . . . . . . . . . . . . . . . . 158

D.4.1 The Integral . . . . . . . . . . . . . . . . . . . . . . 159

D.5 Calculating the Derivatives . . . . . . . . . . . . 162

D.5.1 Single-Point Multiple Approximation . . . . . . . . . . . 162

D.5.2 Numerical Approximation of Derivative . . . . . . . . . . . 164

$\begin{array}{ll}\text { E Laguerre and Jenkins-Traub Algorithms } & 169\end{array}$

E.1 Laguerre Algorithm . . . . . . . . . . . . . . . . . . . . . 169

E.2 Jenkins-Traub Algorithm . . . . . . . . . . . . . . . 170

E.2.1 Stage One: No shift . . . . . . . . . . . . . . . . . . 172

E.2.2 Stage Two: Fixed shift . . . . . . . . . . . . . . 172

E.2.3 Stage Three: Variable Shift . . . . . . . . . . . . . . . 173 
F Control Files

175 


\section{List of Figures}

2.1 Geometry of a light ray bent by gravitational microlensing. . . . . 11

2.2 Geometry of light rays forming the Einstein ring. . . . . . . . . . 12

2.3 Geometry of single lens event, producing two images. . . . . . . . 14

2.4 The separate image and source planes with the two images, source and lens. . . . . . . . . . . . . . . . . . . . 17

2.5 Complex plane showing the distortion of the two images, with the source and the lens. . . . . . . . . . . . . . . . . . . . . 18

2.6 A typical lightcurve for a single lens event, with the source magnification plotted against time in days. . . . . . . . . . . . 19

2.7 Source and image planes for a binary lens event. . . . . . . . . . . 21

3.1 Magnification for a single lens. . . . . . . . . . . . . . . . . . 31

3.2 Lightcurves for 4 microlensing events with various parameters . . 33

3.3 Sky sheet for a single lens. . . . . . . . . . . . . . . . . 36

3.4 Sky sheet for the formation of the Einstein ring. . . . . . . . . . . 37

3.5 Sky sheet for a binary lens system. . . . . . . . . . . . . 38

3.6 Sky sheet for a triple lens system. . . . . . . . . . . . . . . . . . 40

3.7 Critical curves and caustic curves for a binary lens system with equal masses at various lens separations. . . . . . . . . . . . 45

3.8 Critical curves and caustic curves for a binary lens system with unequal masses at various lens separations. . . . . . . . . . . . 45

3.9 The close, intermediate and wide binary cases in parameter space, with the boundaries $d_{I}$ and $d_{I I}$ between each case. . . . . . . . . 46

3.10 Magnification map for a binary lens event. . . . . . . . . . . . . 49

3.11 Three lightcurves for a binary lens event. . . . . . . . . . . . . 50 
3.12 The source plane of a binary lens event. . . . . . . . . . . . . 50

3.13 The image plane of a binary lens event with three equal masses. . 51

3.14 Close up of the image plane of a binary lens event with three equal masses. . . . . . . . . . . . . . . . . 51

3.15 Lightcurve and magnification of each image in a binary lens event. 52

3.16 Lightcurve for event OGLE-2005-BLG-390 . . . . . . . . . 55

3.17 Lightcurve for event MOA-2009-BLG-387 . . . . . . . . . . . 56

3.18 The source plane of a triple lens event with three equal masses. $\quad 57$

3.19 The image plane of a triple lens event with three equal masses. . . 58

3.20 The close up of the image plane of a triple lens event with three equal masses. . . . . . . . . . . . . . . . . . . . . 58

3.21 Lightcurve and magnification of each image in a triple lens event. $\quad 59$

3.22 The root positions and unphysical pairs for a 3 lens system. . . . 59

3.23 The critical curves of a triple lens event with three equal masses. . 62

3.24 The caustic curves of a triple lens event with three equal masses. . 63

3.25 Close up of the caustic curves of a triple lens event with three equal masses. . . . . . . . . . . . . . . . . . . . . 63

3.26 Configuration for a quadruple lens event. . . . . . . . . . . . . 65

3.27 Source plane for a quadruple lens event. . . . . . . . . . . . 65

3.28 Lightcurve for a quadruple lens event. . . . . . . . . . . . . . . 66

4.1 Finite magnification for a single lens event. . . . . . . . . . . . . . 68

4.2 Critical and caustic curves for a triple lens event. . . . . . . . . . 70

4.3 Close up of source track and caustic curves for a triple lens event. $\quad 70$

4.4 Finite magnification for a triple lens event. . . . . . . . . . . . 71

4.5 Limb darkening geometry. . . . . . . . . . . . . . . . . . . . 72

4.6 Limb darkening intensity profiles. . . . . . . . . . . . . . 73

4.7 Polygon area calculation. . . . . . . . . . . . . . . . 75

4.8 Regular polygon vertex placement. . . . . . . . . . . . . . 77

4.9 Chote's dynamic polygon vertex placement. . . . . . . . . 78

4.10 New dynamic polygon vertex placement. . . . . . . . . . . 79

4.11 Limb darkening annuli profiles. . . . . . . . . . . . . . . . . . 81 
4.12 Multiple-point multipole construction. . . . . . . . . . . . 88

4.13 Quadrapole lightcurves for triple lens event. . . . . . . . . . . . 89

4.14 Hexadecapole lightcurves for triple lens event. . . . . . . . . . . . 90

4.15 Magnification for triple lens event. . . . . . . . . . . . . . . . . 94

4.16 Source track and caustic curves for triple lens event. . . . . . . . . 94

6.1 Model lightcurve for MOA-2009-BLG-319. . . . . . . . . . . . . 112

6.2 Magnification map for MOA-2009-BLG-319. . . . . . . . . . . . 113

6.3 Comparison of the model lightcurves for MOA-2009-BLG-319. . . 114

6.4 Model lightcurve for OGLE-2006-BLG-109. . . . . . . . . . . 116

6.5 Caustic curves for MOA-2009-BLG-319. . . . . . . . . . . . 116

6.6 Close-up views of the model lightcurve for OGLE-2006-BLG-109. 117

6.7 Best fitting model lightcurve for OGLE-2006-BLG-109. . . . . . . 119

6.8 Close-up views of the best fitting model lightcurve for OGLE-2006BLG-109. . . . . . . . . . . . . . . . . . . . 119

F.1 Control file used to model MOA-2009-BLG-319. . . . . . . . . . 177

F.2 Control file used to model OGLE-2006-BLG-109. . . . . . . . . 178

F.3 Parameters used to do a small grid search to find a better model for OGLE-2006-BLG-109. . . . . . . . . . . . . . . . 179

F.4 Control file describing a better model for OGLE-2006-BLG-109 . . 180 


\section{Preface}

This MSc project was initially intended to be in two parts. Both of these parts were to involve the development and the use of numerical modelling techniques to investigate two distinct area in astrophysics. The first part of this thesis proposal was aimed at modelling gravitational microlensing events involving multiple lens systems. The particular events of interest were those that contained a planetary mass orbiting a host star. This part of the project ended up growing to fill a majority of the MSc thesis programme, as explained below.

The second part of this proposed project was to carry out modelling of the structure of white dwarf stars. A white dwarf is the final evolutionary stage for the vast majority of stars, and investigating the structure of these slowly cooling stars is a flourishing area in astrophysics research. My supervisor Denis J. Sullivan (DJS) has an active observational programme studying pulsating white dwarf stars, and his collaborators at University of Texas have developed code for modelling white dwarfs and are experts in its use. A relatively recent collaborative programme called MESA (Modules in Experiments in Stellar Astrophysics) has been set up by a group of astrophysicists. This collaborative programme is leading to more general stellar evolution software packages. The intention for the thesis was to investigate these packages. I presented an oral paper on my initial white dwarf modelling activities at the Royal Astronomical Society of New Zealand (RASNZ) annual conference held in May 2011 in Napier.

To date, twenty six extrasolar planets (including ten free floating planets) have been discovered via gravitational microlensing. The raw data from a microlensing event is in the form of a lightcurve, as explained in the body of the thesis. The physical information is obtained from the data by fitting theoretical lightcurves to this observed lightcurve. Generating model lightcurves for planetary lens events 
of interest requires a significant amount of computing power.

The first modelling software designed for gravitational microlensing at VUW (Victoria University of Wellington) was developed by Aarno Korpela. The software developed by Korpela used a method called 'inverse-ray tracing' to generate model lightcurves and this code was run on one of the grid computing systems at VUW. Korpela's work is discussed in his VUW PhD thesis (2007). In 2009, Paul Chote started working with the Korpela code, but the focus of this work shifted to another method for generating theoretical lightcurves, now known as the 'semi-analytical method'. This method is discussed in his VUW MSc thesis (2011).

The gravitational microlensing part of my thesis project was aimed at using and developing the modelling code discussed in Chote's thesis. In particular, the proposed activity was to use the semi-analytical method to accurately compute the lightcurves for multiple lensing systems. This part of the project led to significant work dealing with various numerical accuracy issues.

Of the sixteen planets orbiting a host star that have been discovered via gravitational microlensing, all but two have been found in binary lens events (one star and one planet). The other two planets were found orbiting the same host star, giving a triple lens system. It is not impossible that a system consisting of four lenses might be found in the future, so these systems should also be investigated.

The semi-analytical method investigated in this and in Chote's thesis requires numerically solving a complex polynomial. The polynomial for a binary lens system has degree five, which cannot be solved analytically, and the degree of this polynomial increases quadratically as the number of lenses increases. This thesis discusses how these polynomials can be solved with adequate numerical precision.

Only the research on gravitational microlensing modelling is discussed in this thesis. It was considered inappropriate to include a chapter describing the initial work that was done on white dwarf modelling. 


\section{Acknowledgments}

First of all I would like to acknowledge my supervisor Denis Sullivan (DJS) for his invaluable guidance over the course of this project. Also to the rest of the Optical Astronomy Group at Victoria University, especially Paul Chote for his work on the original mlens2 software and for helping me with the programming aspect of this project, and to Pauline Harris for her valued discussions, editorial work, and much needed emotional support. I would like to thank Marsden Fund for their generous financial support for this work.

Next I would like to thank my family - my Mother, Father and Sister for their selfless support over my years at university, and to my friends, in particular Olex, Waruna, Nick W and the others for their unwavering support, as well as the members of Wellington Youth Choir, New Zealand Youth Choir, and the Choir of Wellington Cathedral of St Paul for their endless encouragement.

I would also like to thank all those in bible study groups for their friendship, spiritual support, and wonderful discussions about life, the universe, and everything. Finally, I would like to thank God for his serenity, courage, and wisdom. I feel honoured and humbled to be studying his beautiful universe. 


\section{Chapter 1}

\section{Introduction to Gravitational Microlensing}

The underlying concepts of gravitational lensing originated in the remarkable works of Sir Isaac Newton, when early ideas about the nature of gravity were being developed. Newton himself promoted the corpuscular theory of light, where light is composed of small discrete particles called "corpuscles" (little particles) which travel in straight lines with finite velocity and possess kinetic energy. The idea that rays of light could be deflected by gravity was first speculated in Newtons third book of Opticks, which contained a set of "Queries" or rhetorical questions that puzzled over the nature of light. The very first of his queries (Newton, 1718) asked, "Do not Bodies act upon Light at a distance, and by their action bend its Rays; and is not this action (caeteris paribus) strongest at the least distance?" (p. 313)

It wasn't until much later that Newtonian mechanics was used to calculate the amount a beam of light would be deflected by the gravity of a star. This was first attempted by French physician and revolutionary Jean-Paul Marat in 1783. However, his calculation significantly overestimated this deflection angle by a large factor (Marat, 1780; Treder and Jackisch, 1981). The next attempt at this calculation was performed by British scientist Henry Cavendish sometime around the late 18 th century to the early 19 th century. The exact year of when he made this calculation is unknown as his paper was not published at the time, however the method he proposed to calculate the deflection angle is presented in the collection of his complete works (Cavendish et al., 1921), and is given as, 
To find the bending of a ray of light which passes near the surface of any body by the attraction of that body. Let $s$ be the centre of body and $a$ a point of surface. Let the velocity of body revolving in a circle at a distance as from the body be to the velocity of light as $1: u$, then will the sine of half bending of the ray be equal to $\frac{1}{1+u^{2}}$. (p. 437)

The first published paper to give a reasonable estimate of the deflection of a light ray grazing the limb of the Sun was by German physicist Johann Georg von Soldner who, in 1801, calculated the angle of deflection as $\omega=0.84^{\prime \prime}$ (Soldner, 1801; Jaki, 1978). However, in the same paper, he alluded that this deflection would be so small, that it would be unobservable.

At this point in time, the idea of light rays being deflected due to gravity was closely tied with the corpuscular theory of light. In fact, even the French physicist and mathematician Pierre-Simon Marquis de Laplace used the corpuscular theory of light to postulate the existence of black holes - bodies so massive and dense that corpuscles of light cannot escape from their surface (de Laplace, 1799; Hawking and Israel, 1989). However, when Thomas Young conducted his double-slit experiment in the early 19th century, the corpuscular theory of light was unable to explain the interferences patterns produced. This significant finding propelled the wave model as the accepted theory of light, thus leaving the corpuscular model of light and the theory that light rays can be deflected by gravity somewhat redundant at this time.

However, a century later, not only was the particle theory of light reconsidered (this time as packets called "photons"), but in an unrelated paper by the same scientist, the idea that light rays could be deflected by gravity was again proposed. This scientist was Albert Einstein.

In his 1915 paper, Einstein used the equivalence principle to develop a new theory of gravity. This new theory predicted that a ray of light would be deflected in a gravitational field. Einstein calculated that the amount of deflection of a light ray passing the Sun was twice the amount predicted by Soldner. Einstein's prediction was validated in 1919 by two expeditions led by Eddington where, 
during a solar eclipse, they measured the amount of deflection of the light passing the Sun from a background star. This became one of the pivotal verifications of Einstein's new general theory of relativity.

Russian physicist Orest Chwolson was among the first to study this 'new' gravitational lensing effect, and in 1924, published a paper (Chwolson, 1924) showing how gravitational lensing could produce multiple images of a star. However, the subject of gravitational lensing received very little attention from the scientific community until 1936, when Einstein published a paper (this time at request of $\mathrm{R}$. W. Mandl) showing how the gravitational lensing effect could make a background star appear as a luminous ring to an observer, if the background star, lensing body and observer are perfectly aligned (Einstein, 1936). This luminous ring is now known as the Einstein ring. However, after estimating the angular radius of this ring to be much less than an arcsecond, which was smaller than the resolving power of telescopes, he stated in this paper, "there is no hope of observing this phenomenon directly." (p. 506)

When Einstein described the phenomenon of the luminous ring, he described it in terms of stars within our galaxy acting as gravitational lenses. However, in 1937, Swiss astronomer Fritz Zwicky suggested that extragalactic objects such as galaxy clusters could also act as gravitational lenses, and would in fact offer a much better chance of observing these gravitational lensing effects (Zwicky, 1937).

This proved to be true, with the first gravitational lensing event to be observed was in the form of a distant quasar lensed by a galaxy. This gravitational lensing effect produced the illusion of 'twin quasars', which was observed in 1979 (Walsh et al., 1979). Later that year Kyongae Chang and Sjur Refsdal proposed that the individual stars in the lens galaxy could act as tiny lenses, making the luminous flux of the quasar's images fluctuate on the order of months (Chang and Refsdal, 1979). These tiny lenses would have Einstein rings with radii on the order of $10^{-5}$ arcseconds, leading Polish astronomer Bohdan Paczyński to coin the term microlensing to describe this phenomenon.

In 1986, Paczyński suggested that gravitational microlensing could be used to 
search for MACHOs (MAssive Compact Halo Objects), a dark matter candidate. He proposed this could be achieved by observing a large number of stars in the Magellanic Clouds. If MACHOs existed and passed in front of the stars, these objects would act as gravitational lenses, amplifying the observed luminous flux from the distant stars (Paczyński, 1986). In reality, the gravitational lens would produce multiple images of the stars, but as suggested by Soldner and Einstein these images would be impossible to resolve, so the only observable effect would be the changing observable flux of the star as the MACHOs moved in front of them. Plotting the changing observed flux over time gives a lightcurve, which can be analysed to place limits on the mass of these MACHOs. The density of these MACHOs would be proportional to the frequency at which these events occur, however more recent observations have shown that MACHOs cannot account for a significant proportion of the dark matter in our galaxy (Tisserand et al., 2007).

The idea that gravitational microlensing could be used to find extrasolar planets was first proposed by Mao and Paczyński (1991). It was shown that the alignment of two stars in the Milky Way galaxy could give a lightcurve similar the "single lens" lightcurves expected for a MACHO event. Any planets orbiting this star could cause dramatic deviations from these single lens lightcurves if the planets were positioned close to the Einstein ring, equivalent to an orbital radius of about $4 \mathrm{AU}$ (the Astronomical Unit - the distance between the Earth and Sun) for a star $4 \mathrm{kpc}$ from Earth. The deviations would occur over the timescale of just a few hours, and the magnitude and time of these changes would depend on the properties of the planet, such as its position and mass. These planetary lightcurves could then be modelled to obtain values for these properties.

A year later, Paczyński founded OGLE (Optical Gravitational Lensing Experiment), a project originally intended to search for MACHOs, but since 2001, has primarily focused on the search for extrasolar planets. This collaboration, along with the New Zealand-Japan collaboration MOA (Microlensing Observations in Astrophysics), have become the principal survey groups in the search for extrasolar planets.

Gravitational microlensing has a number of advantages over the other plan- 
etary detection techniques. While most other methods require observing over a full (or several full) orbit periods of the planet, gravitational microlensing provides a "snapshot" of the planetary system. Since the orbital period of the planet increases with its distance from its host star, most of these other methods cannot detect planets orbiting far from their host star, while gravitational microlensing can. However, perhaps the most significant advantage is that most other techniques depend on the light from the planet's host star, whereas gravitational microlensing does not. Therefore, this technique can not only detect planets orbiting dim or dark stars, such as brown dwarfs, or black holes, but it can also detect "free-floating" planets - planets not gravitationally bound to any star (Sumi et al., 2011).

One of the main disadvantages of this technique is that physical information of interest, such as mass or distances, cannot be directly inferred from the observed data, and only mass ratios or angular distances can be inferred from the lightcurve. The values of mass or distances are normally obtained using statistical estimates.

Another disadvantage with microlensing is the rarity that two stars align. Meaning these events are infrequent, also they are a one-off event that cannot be followed-up by repeated measurements. Therefore, when an event occurs, it is important to obtain as much high quality data as possible during the event.

To ensure each event has an ample number of data points, the microlensing community has developed a two-level structure for observing gravitational microlensing events. MOA and OGLE regularly observe a large number of stars, searching for candidates for microlensing events. When a potential candidate has been discovered, the follow-up collaborations are alerted to this candidate, and these collaborations collect additional observations. These follow-up collaborations include $\mu$ FUN (Microlensing Follow-Up Network), MPS (Microlensing Planet Search), MiNDSTEp (Microlensing Network for the Detection of Small Terrestrial Exoplanets) and Robonet-II.

The follow-up collaborations have smaller telescopes than MOA and OGLE, so unlike these survey groups they cannot observe a large number of stars at any 
one time, however they use their telescopes to observe the potential microlensing candidates to help obtain continual data coverage (Christie, 2006; Tsapras et al., 2009). These microlensing events can last from a few hours up to hundreds of days. To date, the gravitational microlensing technique has discovered ten free-floating planets, as well as sixteen other planets in fifteen planetary systems (Bond et al., 2004; Udalski et al., 2005; Bennett et al., 2006; Beaulieu et al., 2006; Gould et al., 2006; Gaudi et al., 2008; Bennett et al., 2008; Dong et al., 2009; Sumi et al., 2010; Janczak et al., 2010; Miyake et al., 2011; Muraki et al., 2011; Batista et al., 2011; Yee et al., 2012; Bennett et al., 2012; Bachelet et al., 2012).

Once a gravitational microlensing event has ended, the observed lightcurve is then fitted to theoretical models. Single lens events can be easily modelled, however planetary events or multiple star systems are much more difficult to analyse and requires the production of a huge number of theoretical lightcurves along with searches in a large parameter space to find the model that best fits the observed lightcurve. Therefore, when modelling microlensing lightcurves, it is important to generate the theoretical lightcurves quickly, as this decreases the amount of computing time. However, it is also important to calculate these lightcurves accurately to obtain reliable results to best match the observed data.

This thesis builds upon the semi-analytic method developed by Chote (2011). The semi-analytic method works by constructing polynomial called lens polynomials and numerically solves these polynomials the obtain potential image positions for lensing planetary systems with up to three planets orbiting a host star. The theoretical lightcurve can then be generated from these potential image positions. The work presented in this thesis expresses these polynomials in a general form, which can be used to analyse lightcurves for models with any number of planets. This thesis also focuses on a number of numerical techniques and algorithms aimed at improving both the accuracy and speed of numerically solving these polynomials, most notably the Jenkins-Traub and Laguerre algorithms, as well as methods to accurately calculate the lightcurves from the theoretical image positions. The techniques discussed in this thesis have been incorporated into a computer package mlens2, designed and built at VUW (Victoria University of 


\section{Wellington).}

In this thesis, chapter 2 presents an overview of gravitational microlensing, describing the background theory behind this phenomenon and introduces the lens polynomials. This chapter also shows how the Einstein ring forms when there is an alignment of the (background) source star, the lens star, and the observer. Single lens events that form multiple images are also investigated, as well as multiple lensing systems, and polynomials that describe the image positions are derived for these cases.

Chapter 3 discusses the nature of the images produced in microlensing events and investigates how the position of each image relates to the magnification of that image.

Chapter 4 investigates a number of methods to estimate the total magnification of a star (the sum of all the image magnifications) and discusses the advantages and disadvantages of each method.

Chapter 5 introduces numerical algorithms that can be used to solve the lens polynomials. The Jenkins-Traub and the Laguerre algorithms are discussed, along with several numerical techniques often used to make these algorithms run more efficiently. This chapter investigates the speed and accuracy of these algorithms, and introduces the origin-shifting method, developed to reduce the numerical errors in the image positions.

Chapter 6 demonstrates the practical applications of techniques derived in the previous chapters. Also described is how these techniques can be incorporated into the mlens2 package, and uses this package to analyse the microlensing events OGLE-2006-BLG-109 and MOA-2009-BLG-319.

Chapter 7 concludes by discussing the outcomes of this thesis project.

The appendices contain material that is best not included in the main body of the thesis, but contain important information such as tables of data and mathematical equations used in this thesis project.

Appendix A contains the analytical expressions and derivations of the lens polynomial.

Appendix B gives an overview of the Jacobian matrix and its determinant, 
and shows how it is used in microlensing to estimate the image magnification.

Appendix $\mathrm{C}$ contains the analytical expressions and derivations of polynomials that have roots that describe the critical curves.

Appendix D gives a full derivation of the multipole method used to estimate the total magnification of the star.

Appendix E gives a brief overview of the Jenkins-Traub and Laguerre algorithms, which can be used to numerically solve the roots of the lens polynomials.

Appendix F contains the control files used to generate the model lightcurves and to search for the best fitting model using the mlens2 software package. 


\section{Chapter 2}

\section{Microlensing Theory}

Although the full mathematical description of gravitational microlensing is originally derived from the equations of general relativity, these mathematical relationships can be simplified into elementary complex algebra, by making some appropriate assumptions. This allows many physicists and astronomers to understand the fundamental principles behind microlensing without needing a background in the complicated mathematics of differential geometry.

This chapter aims to give a basic overview of the theory behind gravitational microlensing. We start by looking at the case of a single gravitational lens and familarise ourselves with the concept of the Einstein ring. We show how the light from a distance source star forms this ring when the source star, the lensing object, and the observer are perfectly aligned. We then discuss how multiple images are produced, and show how the angular positions of these images are given by an equation called the lens equation.

Configurations with multiple lensing bodies are then introduced and we discuss how the lens equation can be generalised to include these multiple lens configurations. We conclude the chapter by showing how the lens equation for a multiple lens system can be rearranged into a polynomial, where the roots of this polynomial give the positions of the images.

\section{$2.1 \quad$ Einstein Ring}

In Einstein's general theory of relativity, the gravitational field in the vicinity of a massive, uncharged, non-rotating spherical body with mass $M$ can be described 
in terms of a value called the Schwarzschild radius $R_{s}$. The Schwarzschild radius defines the radius of the spherical 'event horizon' for an uncharged, non-rotating black hole with mass $M$. The 'event horizon' is a boundary in space-time, which surrounds the black hole, such that any object including photons of light from inside the event horizon cannot escape to an observer outside.

The Schwarzschild radius for an object is directly proportional to its mass $M$ and does not depend on any other variable quantity. Therefore, $R_{S}$ is a suitable scale to use when comparing gravitational field strengths. The Schwarzschild radius, $R_{S}$, is given by,

$$
R_{S}=\frac{2 G M}{c^{2}}
$$

where $G$ is the gravitational constant and $c$ is the speed of light.

Any physical object must have a radius greater than the Schwarzchild radius. If an object had all its material squeezed into a sphere with a radius smaller than $R_{S}$, then this material would be unable to escape the gravitational influence of the object, and the object would form a black hole with an event horizon of radius $R_{S}$. For very dense objects where the physical radius is close to the Schwarzschild radius, such as neutron stars, the full equations of general relativity are usually required to describe the gravitational field close to these objects. If a ray of light passes close to a dense object, where the gravitational field is strong, the path of the light ray is deflected by a large angle. In these cases, the full relativistic expressions would be required to calculate this deflection.

Most objects, such as our own Sun and other main sequence stars, have a physical radius much larger than the Schwarzschild radius. For example, the Sun has a mass of $2 \times 10^{31} \mathrm{~kg}$, giving a Schwarzschild radius of $3 \mathrm{~km}$. This is much smaller than the $7 \times 10^{5} \mathrm{~km}$ physical radius of the Sun. Objects such as these have relatively weak gravitational fields close to their physical radii, and rays of light passing through these weak gravitational fields will bend by only a small angle. Therefore, the small angle approximation can be applied to transform the full equations of general relativity into much simpler expressions to obtain the angle of deflection of the light ray. 
Using these 'weak-field' equations, it can be shown that a light ray from a distant star (called the 'source' star), which passes close to another star (called the 'lens'), will have its path deflected by an angle $\alpha$ near this lens. This angle of deflection is given by,

$$
\alpha=\frac{2 R_{S}}{b}
$$

where $b$ is the impact parameter - the closest distance of the path of the light ray to the lens.

Since the Schwarzschild radius is many orders of magnitude smaller than the interstellar distances travelled by the light rays, it is reasonable to approximate the lens star as a point, with the bending of the light ray occurring only in the 'lens plane' as shown in Figure 2.1. This is known as the thin lens approximation.

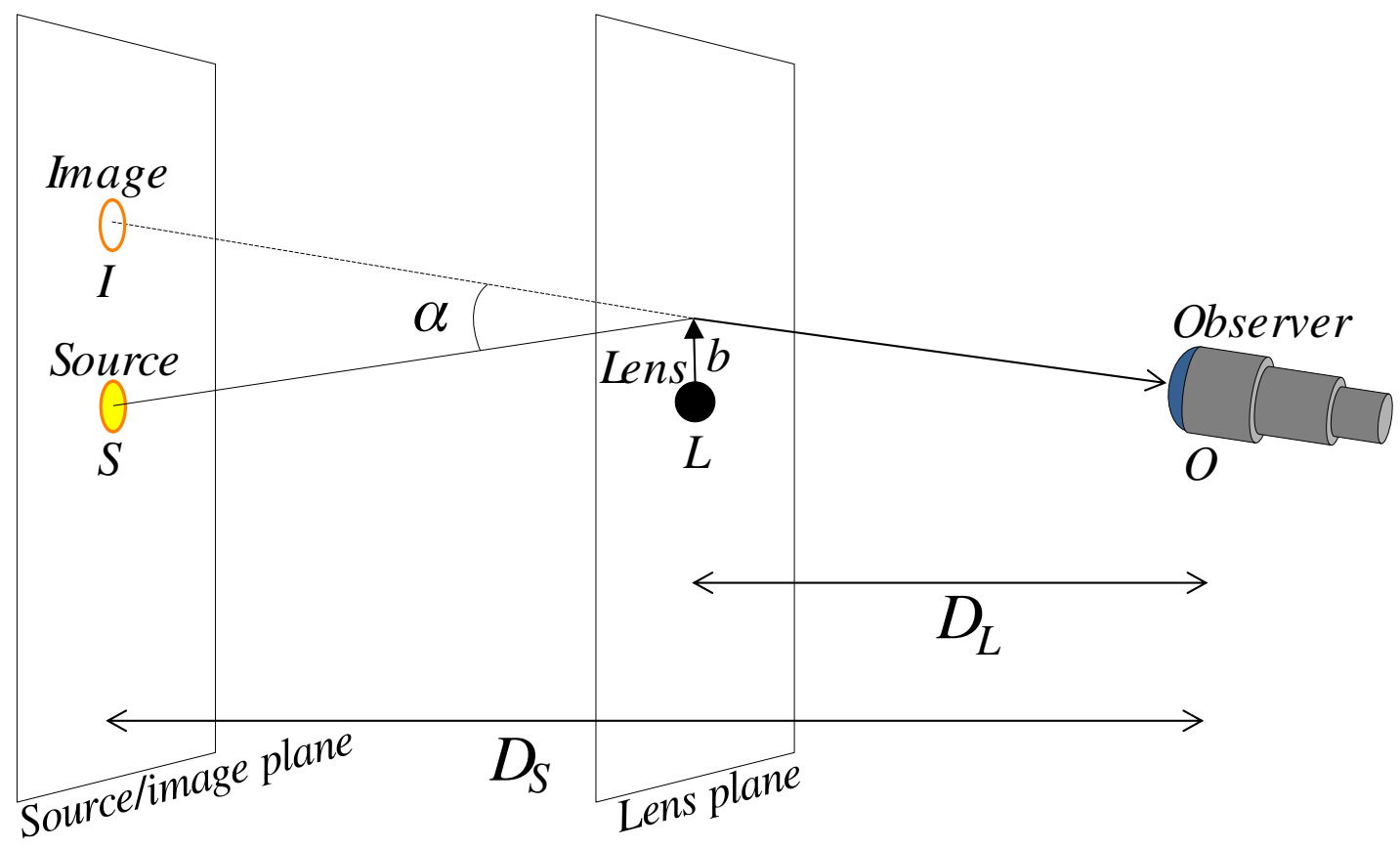

Figure 2.1: Light rays that are emitted by a source star, $S$, are bent by the gravitational field of the lens, $L$, at an angle, $\alpha$, on the lens plane. This causes an image, $I$, to form at a displaced angle, $\alpha$.

In Figure 2.1, a light ray is emitted from a source star, $S$, positioned on the 'source/image plane' at a distance, $D_{S}$ from an observer, $O$. When the light ray passes close to the lens object $L$, the path of the light ray is bent by a small angle $\alpha$, due to the weak gravitational field of the lens, $L$. The lens, $L$, and the point 
where the light ray bends are both positioned on the 'lens plane' at a distance $D_{L}$ from the observer. By tracing the light ray backward in a straight line from the observer to the source/image plane, we find that the observer "sees" this light ray coming from an offset position, $I$, which is an image of the source star. In fact, as discussed later in this chapter, the observer "sees" two images of the star, one due to the bending of light "above" $L$, and one due to the bending of light "below" $L$.

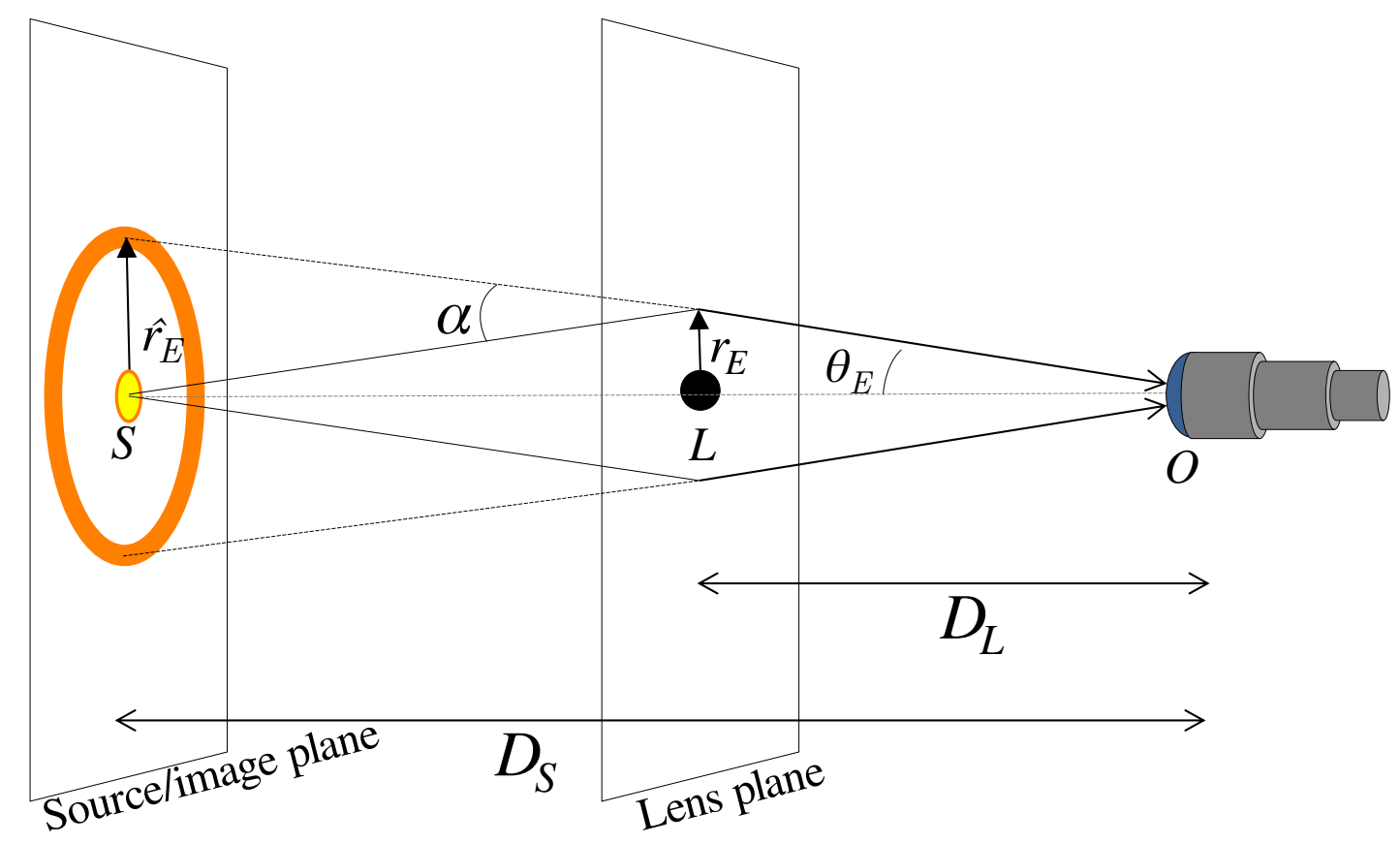

Figure 2.2: When a source, lens and observer are in perfect alignment, the path of the light rays from source star, $S$, are bent by the lens $L$ at an angle $\alpha$ on the lens plane. Due to the circular symmetry of this event, an Einstein Ring is produced.

Figure 2.2 shows the geometry of the light rays when the source star, $S$, lens, $L$, and observer, $O$ are all aligned. In this situation, the geometry is circularly symmetric about the axis $S L O$. By rotating the light rays about $S L O$, the image $I$ is revolved to become a ring on the source/image plane with $S$ at its centre. The formation of this ring was first theorised by Einstein in 1936 and has since become known as the Einstein ring. Following the notation given by Gould (2000), the Einstein radius is represented in the source/image plane by $\hat{r}_{E}$ on the source/image plane. The corresponding radius on the lens plane, found by projecting this ring onto the lens plane from the observer, is represented by $r_{E}$. 
Thus, the light rays in Figure 2.2 have an impact parameter $b=r_{E}$ and arrive at the observer at an angle $\theta_{E}$ to the line $L O$. This angle $\theta_{E}$ is known as the Einstein angle and is related to the Einstein radius by,

$$
\tan \theta_{E}=\frac{r_{E}}{D_{L}}=\frac{\hat{r}_{E}}{D_{S}} .
$$

As mentioned earlier in this chapter, all the angles involved in gravitational microlensing are very small, so the small angle approximation $(\tan \theta \approx \theta)$ can be applied to Equation 2.3 to give $\theta_{E}=r_{E} / D_{L}=\hat{r}_{E} / D_{S}$ and $\alpha=\hat{r}_{E} /\left(D_{S}-D_{L}\right)$. Therefore, the Einstein angle can be expressed as

$$
\theta_{E}=\sqrt{2 R_{S} \frac{D_{S}-D_{L}}{D_{S} D_{L}}}
$$

The typical size of this Einstein angle $\theta_{E}$ can be estimated using the average values for the distances and masses of stars in our galaxy. For example, let us consider a standard microlensing event observed from Earth with a source star in the galactic bulge, $D_{S} \approx 8 \mathrm{kpc}$ from Earth, and a lens star halfway between at a distance $D_{L} \approx 4 \mathrm{kpc}$ from Earth. If the lens star is a Sun-like star with a Schwarzchild radius $R_{S} \approx 3 \mathrm{~km}$, the Einstein angle can be calculated as $\theta_{E} \approx 1$ milliarcsecond.

To determine whether the Einstein ring can be resolved by a telescope on Earth, we can use the Rayleigh criterion. If we consider two point sources of light observed by an instrument with a circular aperture of a diameter $D$, the Rayleigh criterion states that these two point sources of light can only be resolved if the angular displacement $\phi$ between the images, with respect to the observer, obeys the relationship,

$$
\sin \phi \geq 1.220 \frac{\lambda}{D}
$$

where $\lambda$ is the wavelength of the light. The upper limit for the wavelength corresponds to the red end of the electromagnetic spectrum at $\lambda \approx 750 \mathrm{~nm}$. Therefore, given that $\theta_{E} \approx 10^{-3}$ arcseconds, in order to resolve the Einstein ring, the diameter of the telescope lens would have to be at least $189 \mathrm{~m}$, which currently exceeds practical application at this time. 


\subsection{Lens Equation}

The star systems in the Milky Way are in continual motion around the centre of the galaxy, and the distances between these stars is often on the order of parsecs. This means that, the perfect alignment of the source, lens and observer is a very rare event, and even when it does occur, it is only for a short time. Therefore when investigating microlensing events, we need to consider the situation where the source $S$ is not aligned with the lens and observer, but is offset by a small angle. This is shown in Figure 2.3, where the source is offset by an angle $\widehat{S O L}=\beta \neq 0$.

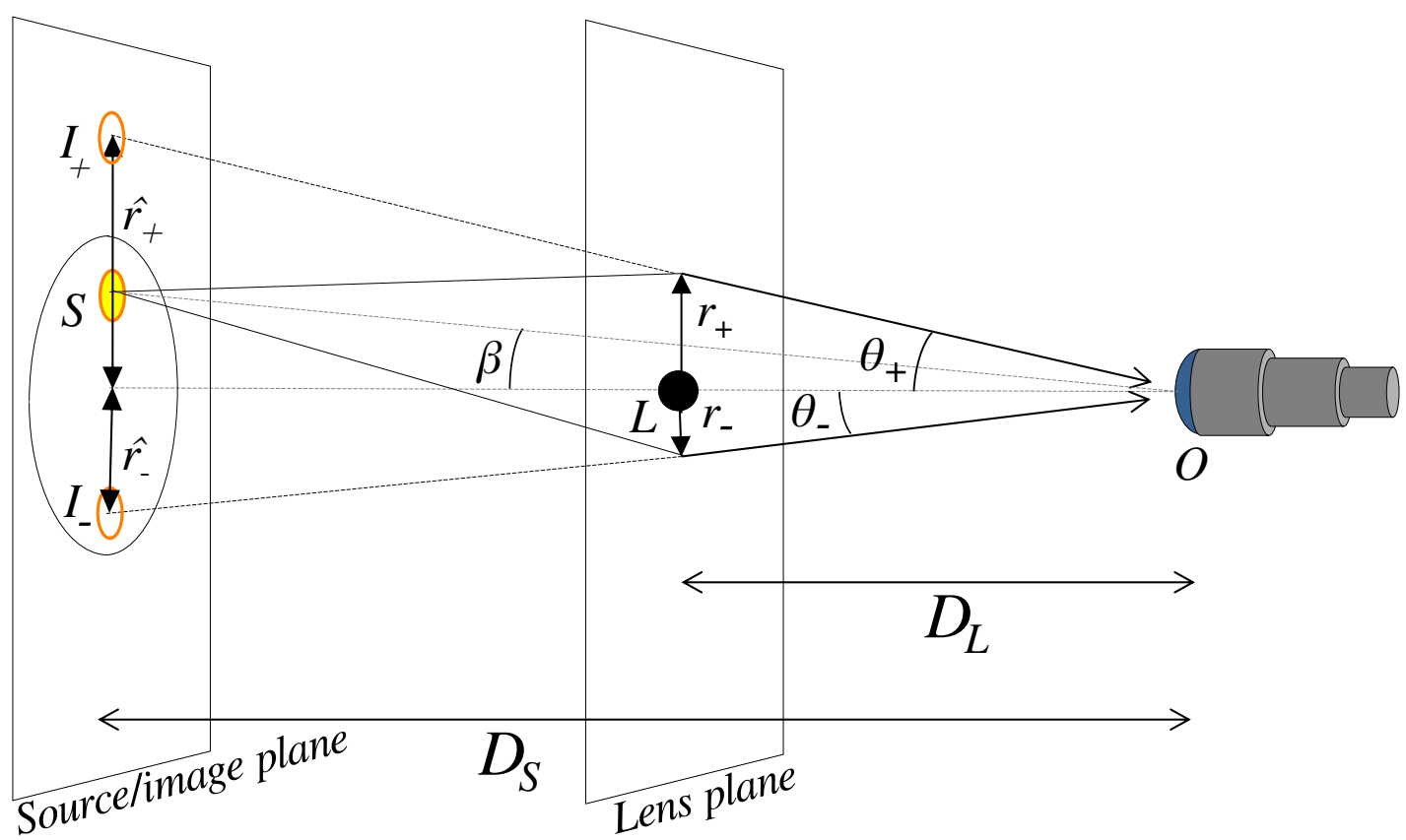

Figure 2.3: Geometry of a single lens event when the source, $S$ is offset at angle $\beta$ from the lens-observer line, $L O$. The light rays from source bend at the lens plane at an angle, $\alpha$, producing the images $I_{+}$and $I_{-}$at the angles $\theta_{+}$and $\theta_{-}$respectively.

The geometry of the light rays shown in Figure 2.3 is not cylindrically symmetric, so the Einstein ring does not form. However, light rays from the source star will bend at the distances $r_{+}$and $r_{-}$, above and below the lens respectively. By tracing the light rays backward in a straight line from the observer to the source/image plane, we find that the observer will "see" two images $I_{+}$and $I_{-}$ on the source/image plane. These images are positioned above and below the line $O L$, at the distances $\hat{r}_{+}$and $\hat{r}_{-}$respectively, with angular displacements $\widehat{I_{+} O L}=\theta_{+}$and $\widehat{I_{-} O L}=\theta_{-}$. Note that the images and source are collinear on 
the source/image plane.

As mentioned earlier in the chapter, the angles involved in microlensing events are often on the order of milliarcseconds. Therefore, the small angle approximation can be applied to the geometry of the event shown in Figure 2.3. Through this approximation, the positions of the images $\hat{r}=\hat{r}_{+}, \hat{r}_{-}$can be related to their respective angles $\theta=\theta_{+}, \theta_{-}$through the following geometrical relationships.

$$
\begin{aligned}
\alpha\left(D_{S}-D_{L}\right) & =\hat{r}-s, \\
\beta D_{S} & =s \\
\theta D_{L} & =\hat{r} .
\end{aligned}
$$

By combining Equations 2.5 - 2.7, a single equation can be derived that relates the angle of the images $\theta=\theta_{ \pm}$with the angle of the source $\beta$ and the Einstein angle $\theta_{E}$. This equation is called the lens equation,

$$
\beta=\theta-\theta_{E}^{2} \frac{1}{\theta}
$$

This equation has two solutions $\theta=\theta_{+}, \theta_{-}$, which gives the angular positions of the two images. These solutions can be found by rearranging Equation 2.8 into a quadratic equation and analytically solving it to obtain,

$$
\theta_{ \pm}=\frac{\beta}{2} \pm \sqrt{\frac{\beta^{2}}{4}+\theta_{E}^{2}} .
$$

From Equation 2.9 it can be shown that the two angles of the images $\theta_{+}$ and $\theta_{-}$are non-zero, real and distinct, for any non-zero real value for the source angle $\beta$. This confirms the notion that the images and source are collinear on the source/image plane. It is interesting to note that $\beta=\theta_{+}+\theta_{-}$. In other words, the angular distance between the two images is always equal to the angular distance between the source and the lens, and the angular distance between $I_{-}$and $L$ is always equal to the distance between $I_{+}$and $S$.

In the case where $\beta \gg \theta_{E}$, it can be shown that $\theta_{+} \approx \beta$ and $\theta_{-} \approx 0$. Chapter 3 shows that as $\theta_{-} \rightarrow 0$, the magnification of image $I_{-}$goes to zero, so that this image is is no longer visible. Therefore, when the angular displacement between 
the source star and the lens is large, the only significant image is $I_{+}$, which is in the same location as the source star. In other words, the lensing mass has no significant effect on the light rays.

In the other limit where $\beta \rightarrow 0$, it can be shown that $\theta_{ \pm} \rightarrow \pm \theta_{E}$. This corresponds to the formation of the Einstein ring when the source star, lensing mass and observer are perfectly aligned.

\subsection{Complex Representation}

While the positions of stars within our galaxy can be described in terms of 3dimensional positions, an observer sees these positions projected onto the 2dimensional plane of the sky. Therefore similarly, the angular positions of the images, source and lens can also be represented on a 2-dimensional plane. On this 2-dimensional plane, the Einstein angle $\theta_{E}$ is represented by the Einstein radius $R_{E}$, and all the image and source positions can be scaled by this value. When everything is scaled in this way, the Einstein ring becomes a circle of unit radius, and the image and source positions can be defined as $z_{ \pm}=\frac{\theta_{ \pm}}{\theta_{E}}$ and $w=\frac{\beta}{\theta_{E}}$ respectively. If these scaling definitions are applied to Equation 2.8, then the lens equation becomes,

$$
w=z-\frac{z}{|z|^{2}}
$$

Since the stars are continually moving within our galaxy, it is important to consider the relative motion of the source star to the lens star when investigating microlensing events. To analyse the relative motion of the source, the image and source positions can be represented by the Cartesian coordinates $z=(x, y)$ and $w=(u, v)$ respectively. These positions can alternatively be expressed in terms of their complex coordinates $z=x+i y$ and $w=u+i v$, as first suggested by Witt (1990). In this representation, the lens equation can be expressed as,

$$
w=z-\frac{1}{\bar{z}}
$$


The lens equation expressed in Equation 2.11 describes a function mapping points on the image plane $z$ to points on the source plane $s$. The separate source and image planes are demonstrated in Figure 2.4. This diagram shows the positions of the images $I_{+}$and $I_{-}$represented by the blue and red dots respectively at distances $z_{+}$and $z_{-}$from the origin respectively, and the source $S$ represented by the orange dot at distance $w$ from the origin. The lens $L$ is represented by the black dot at the origin, and the Einstein ring by the black ring with unit radius.
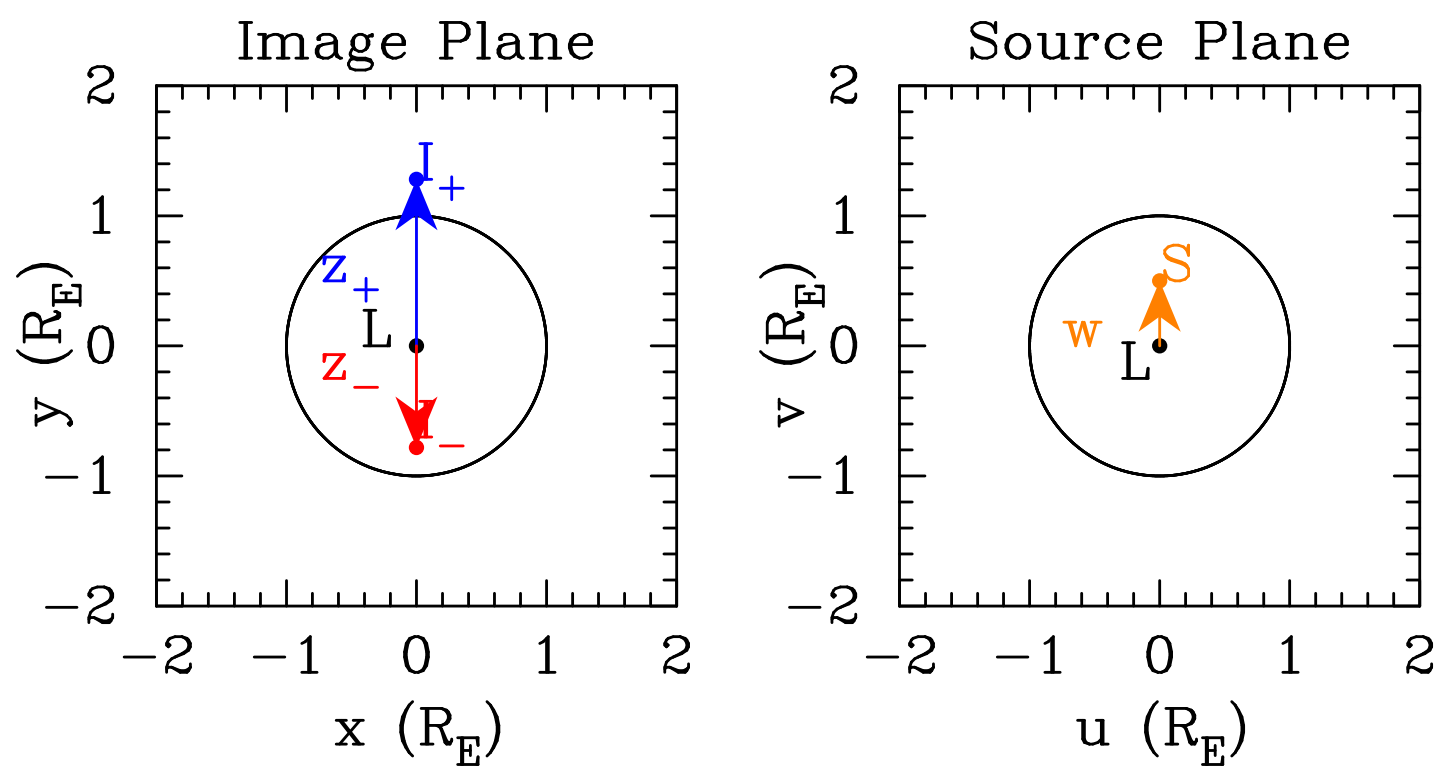

Figure 2.4: The image and source planes, with the source $S$ at a distance $w$, and the two images, $I_{+}$, at position $z_{+}$, and $I_{-}$, at position $z_{-}$. Both these planes have the lens $L$ positioned at the origin.

In the single lens case, the lens equation can be rearranged and solved analytically, and the image positions given by the expression,

$$
z_{ \pm}=\frac{w}{2} \pm \sqrt{\frac{w^{2}}{4}+1}
$$

Since the lens plane is circularly symmetric, we can, without loss of generality, choose the $u$ direction as the direction of motion of the source, with a stationary lens at the origin. As the source moves relative to the lens, the images will also move in such a way that the source, lens and images are always collinear on this plane. This is presented in Figure 2.5, which shows a superposition of the source and image planes, where the source star is represented by an orange circle, and 
the images are represented by the blue and red shapes.

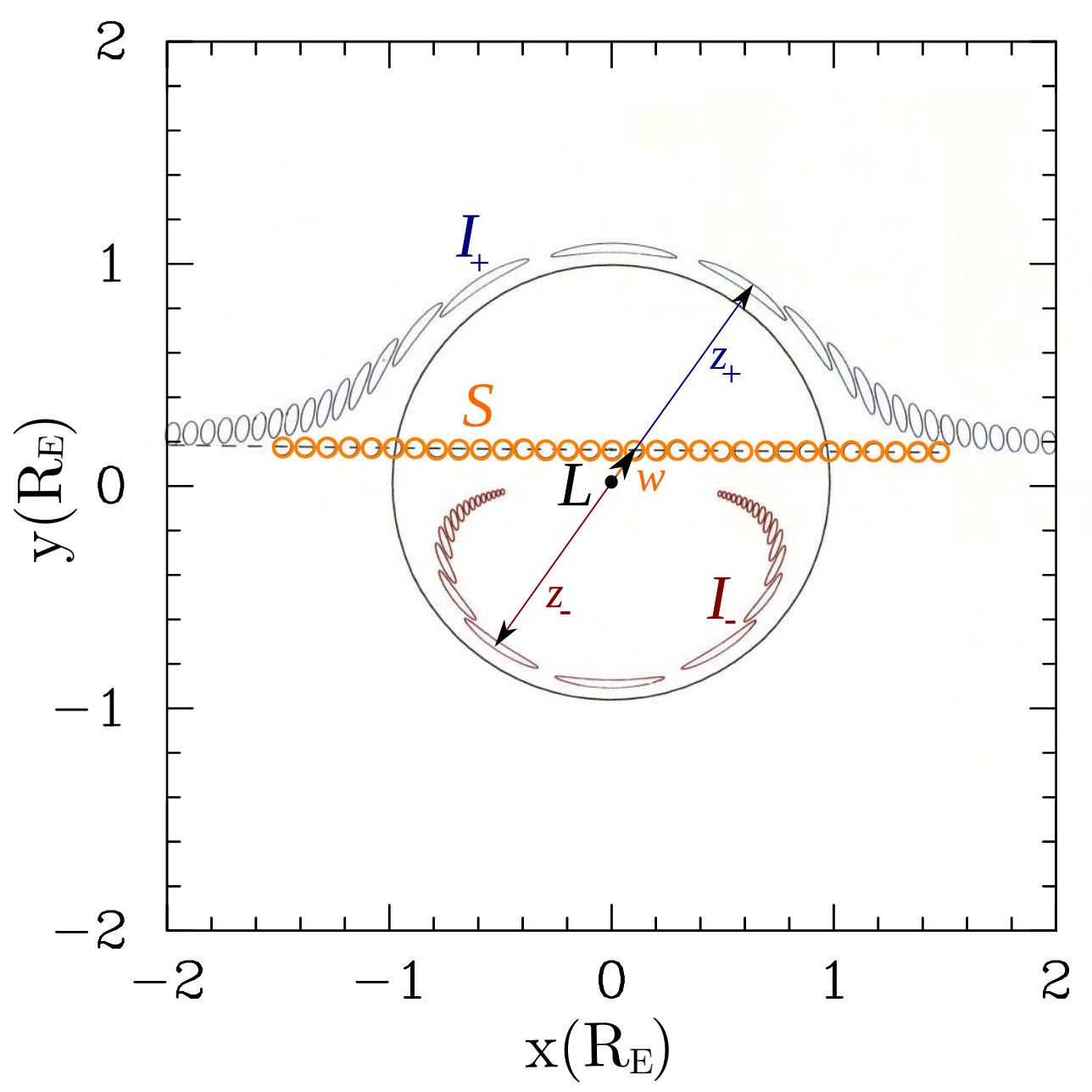

Figure 2.5: Superposition of the source and image planes, showing the source, $S$, lens, $L$ and (distorted) images $I_{+}$and $I_{-}$, for a series of source positions. The source positions are represented by the orange circles, and the blue and red shapes represent the images $I_{+}$and $I_{-}$ respectively.

While the deflection of light rays does not change the intrinsic brightness of the source, it does distort the shape of the images, as demonstrated in Figure 2.5. Therefore, the 'luminous flux' of each image, which is defined as the brightness of the image multiplied by its 'solid angle', does change.

For any gravitational microlensing event, the angular separation between images is of the order of the Einstein angle. Therefore, as mentioned previously, the images that are formed by the alignment of two stars in the Milky Way Galaxy cannot be resolved using any practical telescope on Earth. However, the observed total flux, which is simply the sum of the flux from all the images, can be measured by telescopes on Earth. The observed total flux is directly related 
to the position of the source, and as the source moves relative to the lens, the observed flux will change over time. Therefore, microlensing events can only be observed from the lightcurve, which is a plot of magnification over time, where the magnification is defined as the ratio of the total observed flux of the source to the flux of the source when unaffected by the lens. Figure 2.6 presents a 'typical' example of a lightcurve from a single lens event.

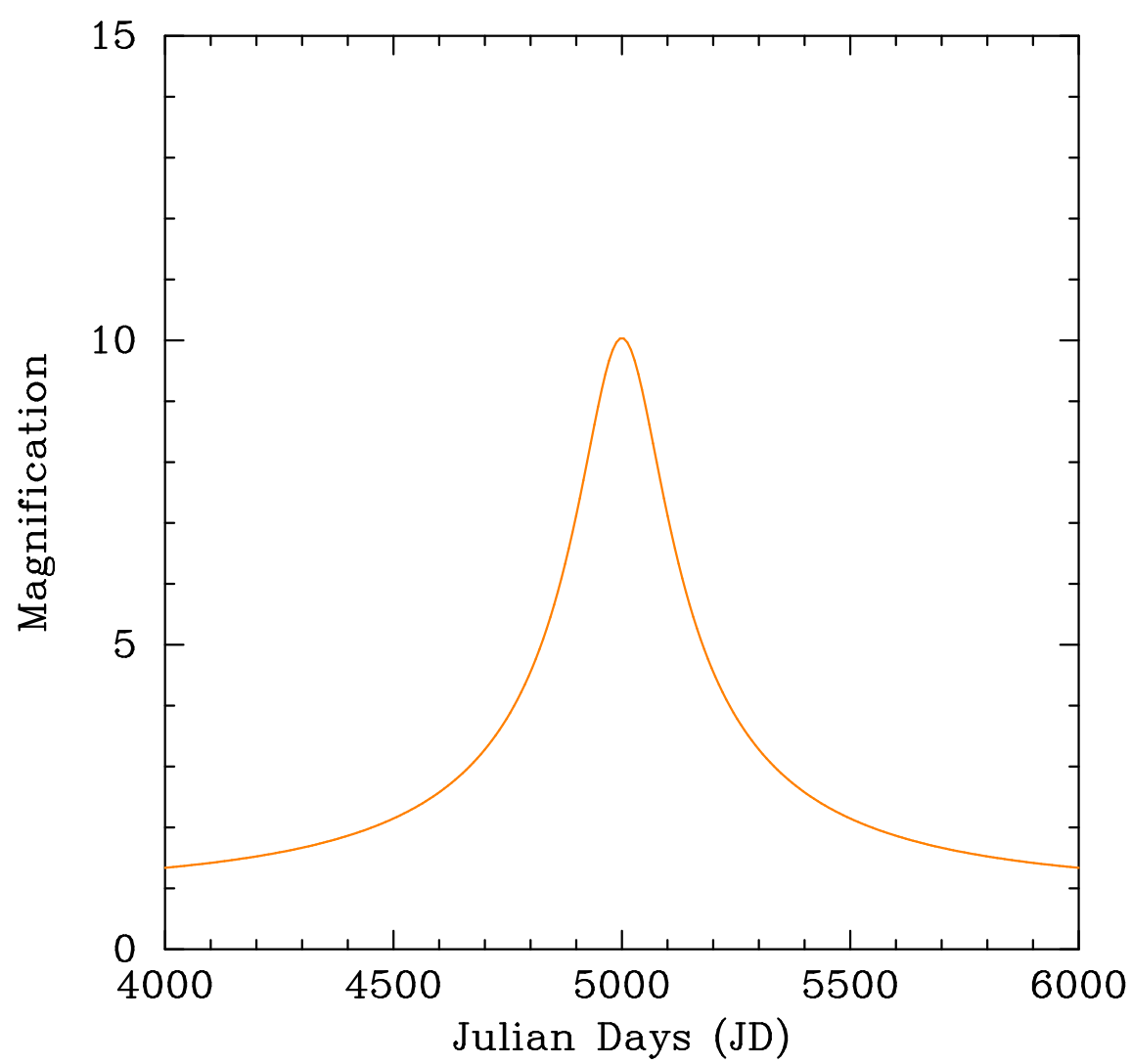

Figure 2.6: A typical lightcurve for a single lens event, with the source magnification plotted against time in days.

Observed microlensing events are analysed by generating a large number of theoretical lightcurves from various models and finding the model that fits best with the observed lightcurve. In these theoretical microlensing models, the magnification of the source can only be calculated once the image positions are known. The following sections of this chapter focus on the mathematical equations used to find these image positions. Methods for calculating the magnification from these image positions are presented in Chapters 3 and 4. 


\subsection{Multiple Lenses}

One of the main reasons for observing and analysing microlensing events is to detect extrasolar planets. In order to analyse these events, the lens equation given in Equation 2.8 must be extended to include multiple lens systems.

In the previous section, the origin of the complex plane was chosen, without loss of generality, to be at the position of the lens. However, in a multiple lens system, there are two or more lenses. Therefore, before the lens equation can be extended to multiple lens systems, it needs to be modified so it describes microlensing events for any arbitrarily chosen origin.

If the origin is chosen in such a way that the lens is at position $r$ from this origin, the source and image positions can be transformed by $w \rightarrow w-r$ and $z \rightarrow z-r$ respectively. Under these transformations, Equation 2.11 becomes,

$$
w=z-\frac{1}{\bar{z}-\bar{r}}
$$

When the lens equation is extended to a system with any integer $N$ number of lenses, Equation 2.13 becomes

$$
w=z-\sum_{j=1}^{N} \frac{\epsilon_{j}}{\bar{z}-\bar{r}_{j}}=z-\frac{\epsilon_{1}}{\bar{z}-\bar{r}_{1}}-\frac{\epsilon_{2}}{\bar{z}-\bar{r}_{2}}-\cdots,
$$

where $r_{j}$ is the distance of the $j$ th lens from the origin, and $\epsilon_{j}=M_{j} / M_{T}$ is the mass fraction of this lens where $M_{j}$ is the mass of the $j$ th lens and $M_{T}=\sum_{j=1}^{N} M_{j}$ is the total mass of all the lenses.

The lens equation shown in Equation 2.14 describes a function mapping points on the image plane $(z=x+i y)$ to points on the source plane $(w=u+i v)$. This is demonstrated in Figure 2.7. Every point on the image plane maps to a unique point on the source plane. However, when the equation is inverted, every point on the source plane corresponds to multiple points on the image plane. The number of image points that correspond to the source point depends on the number of lenses, the configuration of these lenses, and the position of the source with respect to this configuration. 


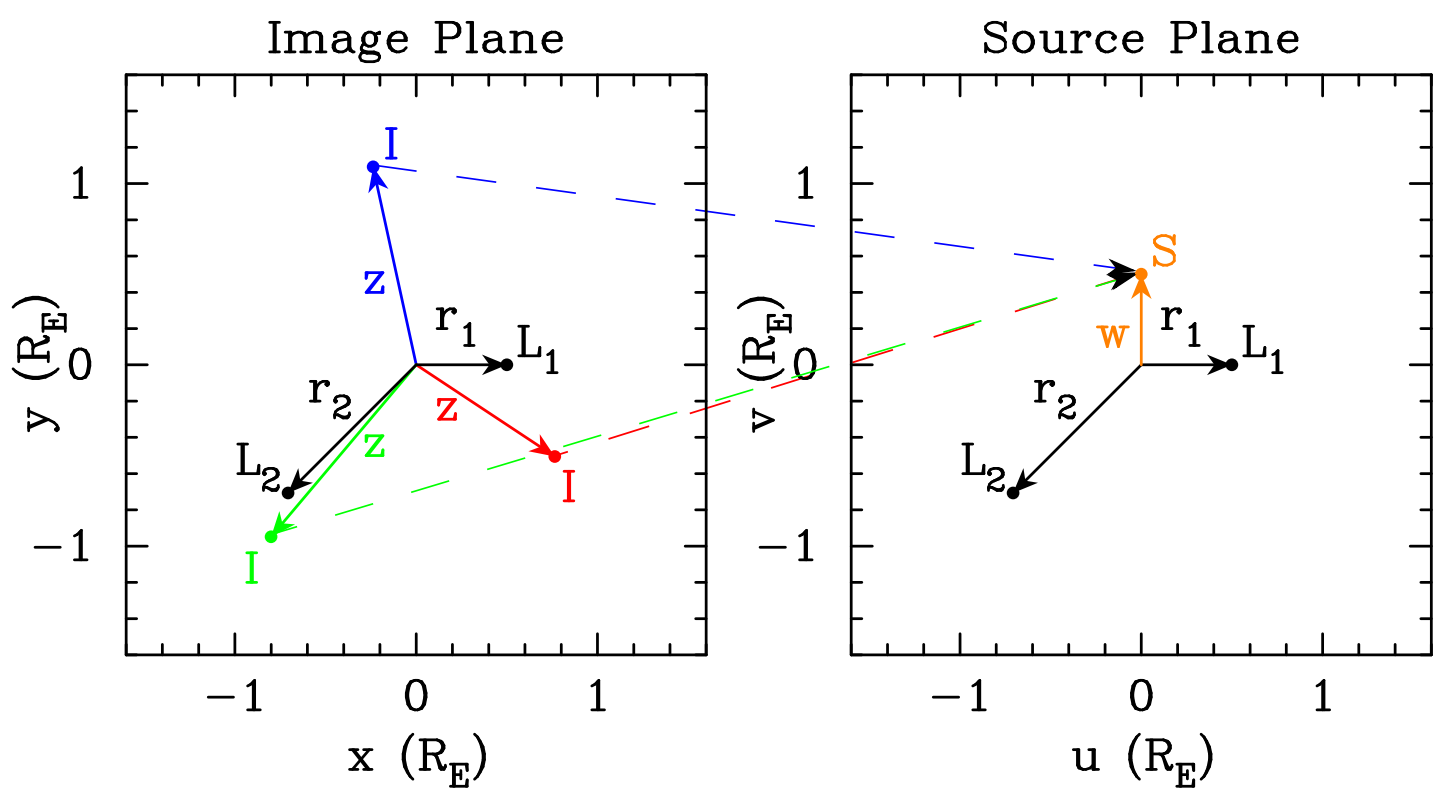

Figure 2.7: Source and image planes for a binary lens event. The two lenses, $L_{1}$ and $L_{2}$, are located at positions, $r_{1}$ and $r_{2}$, respectively on both planes. Each image $I$ at position $z$ on the image plane corresponds to the source $S$ at position $w$ on the source plane. This transformation from image to source plane can be obtained via the lens equation (Equation 2.14).

While the lens equation for a single lens can easily be inverted and solved analytically, solving the lens equation for multiple lenses is much harder, as there is no analytical solution to this equation. The difficulty of inverting the lens equation has led many microlensing modellers to use a brute force approach known as inverse-ray tracing. This approach works by iterating through a large number of potential image positions and 'inverse-ray tracing' these positions to the source plane by substituting these image positions $z$ into the lens equation in Equation 2.14. The positions on the image plane that return the original source position $w$ are recorded as physical image positions, whereas the positions that do not are discarded. The process follows directly from the reversibility of light, whereby any light ray travelling from the source to the lens plane or observer will follow exactly the same path when going from the observer or the lens plane back to the source.

One way of implementing this approach (sometimes called ray-shooting) is by working with a finite source (often represented by a disk) at some position on the source plane. The image plane is then represented by a pixel grid of all potential image positions. The potential image positions are then iteratively substituted 
into the lens equation to find the pixels that correspond to the source disk. The magnification of the source is then estimated as the ratio of the total area of the successful pixels in the image plane to the area of the source disk. A lightcurve can then be constructed by iterating through a series of source positions on the source plane. Limb darkening effects (discussed in Chapter 4) can also be included by 'weighting' these pixels.

This approach clearly requires a large number of calculations, since a large number of potential image positions are tested. Therefore, this approach requires significant computational resources like grid computing systems. However, this approach can be made more efficient by subdividing the image plane into successively smaller regions and eliminating regions from the search by testing the rays around the perimeter of each region.

Inverse ray tracing can be made more efficient through the use of tools such as magnification maps. Magnification maps are generated by representing the image plane by a fine grid of all potential image positions, which are inverse-ray traced to the source plane. The image positions are then recorded in such a way that the ray density on the source plane can be determined. A lightcurve can therefore be constructed by moving a finite source disk along a path and determining the source magnification at each source position by measuring the number of included rays. While this works well for a fixed lens configuration, when the model contains orbital lens motion, the lens configuration varies through time, and a new magnification map is required for each interval of time, significantly increasing the amount of computational time.

The alternative method to finding the image positions is to invert the lens equation analytically. This can be achieved by rearranging it into an $N^{2}+1$ degree polynomial. However, even a lens configuration with two lenses gives a polynomial with 5th degree, which cannot be solved analytically. Therefore, the polynomials must be solved numerically using polynomial root-finding algorithms. The numerical techniques that can be used to find these roots are discussed in Chapter 5.

The method of rearranging the lens equation into a polynomial in complex 
coordinates was first demonstrated in the binary lens case by Witt (1990) and has since become known as the semi-analytical method (Chote, 2011). However, as more lenses are included, the expressions for the coefficients of the polynomials become increasingly lengthy and more difficult to derive. A method to derive these expressions in the triple lens was developed by Rhie (2002) and this method can be extended to any number of lenses without much difficulty. An overview of this method is given in the following section and the full derivation is presented in Appendix A.

\subsection{Semi-Analytical Method}

In order to rearrange the lens equation for any $N$ lens system into $N^{2}+1$ polynomial with respect to $z$, the $\bar{z}$ terms need to be removed. This can be achieved by first rewriting Equation 2.14 and its complex conjugate as follows,

$$
\begin{aligned}
z-w & =\sum_{j=1}^{N} \frac{\epsilon_{j}}{\bar{z}-\bar{r}_{j}} \\
\bar{z}-\bar{w} & =\sum_{j=1}^{N} \frac{\epsilon_{j}}{z-r_{j}} .
\end{aligned}
$$

To simplify the lens function, we can represent Equation 2.16 in terms of two polynomials $G$ and $H$, such that,

$$
\begin{aligned}
G & =\sum_{j=1}^{N} \epsilon_{j} \prod_{i \neq j}\left(z-r_{i}\right), \\
H & =\prod_{i=1}^{N}\left(z-r_{j}\right) .
\end{aligned}
$$

We can eliminate the $\bar{z}$ terms in the lens equation, we can rewrite Equation 2.16 in terms of $G$ and $H$, and substitute it into Equation 2.15 to give,

$$
z-w=\sum_{j=1}^{N} \frac{\epsilon_{j}}{\frac{G}{H}+\bar{w}-\bar{r}_{j}}
$$


The polynomial $H$ has degree $N$, whereas the polynomial $G$ has degree $N-1$. By defining the variable $\varpi_{i}=\bar{r}_{i}-\bar{w}$, Equation 2.19 can be rearranged to give,

$$
0=(z-w) \prod_{i=1}^{N}\left(G-\varpi_{i} H\right)-\sum_{j=1}^{N} H \epsilon_{j} \prod_{i \neq j}\left(G-\varpi_{i} H\right) .
$$

The polynomial described in Equation 2.20 can be separated into two distinct parts: the first half of the equation, which is the product $\prod_{j=1}^{N}\left(G-\varpi_{j} H\right)$, and the second half of the equation, which is the sum $\sum_{j=1}^{N} \epsilon_{j} \prod_{j \neq i}\left(G-\varpi_{i} H\right)$. These two parts can be considered analogous to polynomials. By defining the variable $\pi_{i}=z-\varpi_{i}$, the polynomials $X$ and $V$ can be constructed in a similar way to $H$ and $G$ respectively, such that the first part of Equation 2.20 is analogous to $X$, while the second part of Equation 2.20 is analogous to $V$ as follows,

$$
\begin{aligned}
X & =\sum_{i=0}^{N} X_{i} z^{i}=\sum_{j=1}^{N} \epsilon_{j} \prod_{i \neq j}\left(z-\varpi_{i}\right), \\
V & =\sum_{i=0}^{N} V_{i} z^{i}=\prod_{j=1}^{N}\left(z-\varpi_{j}\right) .
\end{aligned}
$$

In this analogy, the polynomial from Equation 2.20 would be analogous to $0=(z-w) X-V$. Therefore, we can define a polynomial $W$ such that,

$$
W=w X+V
$$

Equation 2.20 can therefore be expanded and rewritten in the form,

$$
0=z \sum_{i=0}^{N} G^{i} H^{N-i} X_{i}-w \sum_{i=0}^{N} G^{i} H^{N-i} W_{i} .
$$

Note that the $H$ polynomial has degree $N$ and is multiplied by itself at most $N$ times, giving the $H^{N}$ polynomial a degree of $N^{2}$. $H^{N}$ is then multiplied by $z$ in the first part of Equation 2.24, giving this polynomial a degree of $N^{2}+1$.

The expressions for the coefficients of Equation 2.24 can be found by expanding the polynomials $G$ and $H$. The term $\eta_{i, k}$ can then be defined as the $k$ th coefficient of the polynomial product $G^{i} H^{N-i}$,

$$
\eta_{i, n}=\left[G^{i} H^{N-i}\right]_{n}
$$


By combining Equations 2.24 and 2.25, we can represent the polynomial in the form,

$$
0=\sum_{n=0}^{N^{2}+1} c_{n} z^{n}
$$

where the coefficients of this polynomial are given by the expressions,

$$
c_{n}=\sum_{i=0}^{N} \eta_{i, n-1} X_{i}-\sum_{i=0}^{N} \eta_{i, n} W_{i} .
$$

This polynomial is known as the 'lens polynomial'. While this equation can be used to solve for any $N$ number of lenses, the expressions for the coefficients become increasingly lengthy as the number of lenses increases. To date, only one microlensing event has been published that required a model with a three lens configuration (Gaudi et al., 2008) and none that required four or more lenses in the model. While it is possible that a model with four lenses (most likely a star plus three planets) might be required in the future, it seems unlikely that a system with five or more planets will be discovered via microlensing in the immediate future. Therefore, this thesis focuses on configurations with no more than four planets.

\subsection{Number of Images}

Although the lens equation for a system with $N$ lenses can be rearranged into a polynomial with degree $N^{2}+1$, not all of the $N^{2}+1$ roots always correspond to physical images; the set of physical images is only a subset of the roots. A point $z$ on the image plane corresponds to a physical image if and only if this point inverse-ray traces to the source position $w$ whenever it is substituted back into lens equation (Equation 2.14). The roots that do not inverse-ray trace to the source position are unphysical. For a configuration with $N$ lenses, the number of images is always $N+1+2 k$ where $k$ is an integer (Witt, 1990). The specific value for $k$ depends on the position of the source relative to the lens configuration. An upper limit to the number of images was set by Rhie (2001), who argued that the number of images never exceeds $5(N-1)$ when $N>1$. 
For a multiple lens configuration, the number of images may change as the source position moves along a path (known as the source track) on the source plane. As this number changes, the physical images may appear and disappear along the loci of points on the image plane called the 'critical curves'. The corresponding loci of points on the source plane (by inverse-ray-tracing these points to the source plane via the lens equation) are called the 'caustic curves'. The two images appear or disappear at the critical curves on the image plane whenever the source crosses a caustic curve on the source plane. The natures of the critical and caustic curves are discussed in more detail in the following chapter.

To determine the behaviour of the images when the source star is far from the lens positions, we can take the limit $w \rightarrow \pm \infty$. When this limit is applied to Equation 2.14, the lens equation becomes,

$$
z-\sum_{j=1}^{N} \frac{\epsilon_{j}}{\bar{z}-\bar{r}_{j}} \rightarrow \infty .
$$

This equation corresponds to either $z \rightarrow \pm \infty$ or $-\epsilon_{j} /\left(z-r_{j}\right) \rightarrow \pm \infty$, where $j=1,2, \cdots$. The latter corresponds to when $z \rightarrow r_{j}$. Therefore, in this limit, there is one (physical) image located near the source position $w$ and one (physical) image located near each lens position $r_{j}$, giving a total of $N+1$ (physical) images.

When this limit is applied to the lens polynomial presented in Equations 2.26 and 2.27, it can be shown that the polynomial is reduced to,

$$
0=(z-w) \prod_{j=1}^{N}\left(z-r_{j}\right)^{N}
$$

The proof of this result is shown in Appendix A, Section A.3. This shows that there is one root near the source, $w$, and $N$ roots near each lens position, $r_{j}$. Therefore there are $N-1$ unphysical roots near each lens. This result becomes important in subsequent chapters, where the identification of a root as being physical or unphysical depends on the accuracy of the numerical polynomial solver. 


\subsection{Orbital Motion}

For most microlensing events, the relative motion of the source star, lens, and observer can be represented by uniform motion unaffected by any forces. Thus the projected motion of the source relative to the lens as viewed by the observer can be modelled by rectilinear motion - a straight line path at a constant speed.

For a single lens microlensing event, this leads to the lightcurve presented in Figure 2.6, which results from the changing magnification of the source measured by the observer. The microlensing survey groups (MOA and OGLE) use this lightcurve shape to initially identify potential microlensing events from the other causes of stellar variability. Any deviations from the single lens lightcurve shape may imply a multiple lens system (a star plus planet(s) are of most interest) and in this case the follow-up networks are required to help observe and characterise the nature of these deviations.

However, for some microlensing events, orbital (accelerated) motion of the observer, the source, or the lens system may have a significant on the lightcurve, and this orbital motion needs to be included in the models.

The most common type of orbital motion required in models is the accelerated motion of the observer, as the Earth orbits around the Sun. If the time scale of the microlensing event is long enough, the orbital acceleration of the Earth leads to apparent non-rectilinear motion of the source track and a consequent distortion of the lightcurve. This effect is known as microlensing parallax.

In a similar way, any orbital motion of the source star around a companion (which may be unseen) will produce effects similar to parallax. This effect is known as 'xallarap', as it can be thought of as the reverse of parallax. However, while parallax can be modelled with only two free parameters since the orbital period and radius of the Earth are known, the details of the hypothesised orbital motion of the source in xallarap are unknown and need to be included as free parameters in the process of modelling the lightcurve. Nevertheless, several microlensing events have been modelled using xallarap, as well as the better characterised parallax effect.

The third type of orbital motion is that of the lens system itself in the case 
of multiple lenses. The components of a multiple lens system orbit around their common centre of mass, and depending on timescales, this can have an observable impact on the lightcurve. At least one high profile microlensing event required this effect to be included (Gaudi et al., 2008). This type of orbital motion is different from parallax and xallarap, since it does not change the apparent motion of the source; it only changes the positions of the lenses. Lens motion is modelled simply by modifying the lens positions $r_{j}$ as a function of time.

When lens motion is included in the models, the lens configuration changes so the coefficients for the polynomials $G, H, X, V$ and $W$ in Equations 2.17, 2.18, 2.21, 2.22 and 2.23 need to be recalculated at each time interval. However, if there is no lens motion, the lens positions $r_{j}$ do not change, so the polynomials $G$ and $H$ only need to be calculated once for each lightcurve, thus reducing computing time. For more details on orbital motion, see Chote (2011). 


\section{Chapter 3}

\section{Image Topology}

In Chapter 2, we gave the analytical theory of microlensing and showed how to derive polynomials, which have roots that are supersets of the image positions. That chapter also explained how to determine whether a root from this equation is a physical image or not. In this chapter, we will look into how to use these image positions to calculate the magnification of a single point source position, and how this can be used to calculate a lightcurve for a given lens configuration.

This chapter starts by looking at the lens equation introduced in Chapter 2, using it to investigate the topology of the image plane, and showing how to find the magnification of each (physical) image for a point source. We introduce the concepts of critical curves, caustic curves, domains, and parity and show how these relate to the number and magnification of the (physical) images. We then show how to construct polynomials with roots that correspond to the critical curves, and we use these polynomials to analyse the behaviour of the critical and caustic curves for binary and higher lens configurations. We conclude this chapter by focusing on configurations with multiple planets, investigating how the position of each image affects its contribution to the total source magnification, and showing how the caustic curves and lightcurves from these events can be approximated to first order approximation by several independent binary planetary events. 


\subsection{Point Source Magnification}

As mentioned in Chapter 2, the images cannot be resolved by any practical telescope on Earth; only the observed flux from the source can be measured. The observed flux is defined as the apparent brightness multiplied by the solid angle of the image (or the area of the image when represented a dimensional plane). Although the lensing does not affect the apparent brightness of the star, it does distort the shape, and this distortion in shape will change the area of the projection of the source on the image plane. By comparing the area of the images during a gravitational microlensing event with the area of the source when unaffected by the lenses, we can calculate the magnification of the source star.

Only the observed flux can be measured from a single observation; the area of the source and images cannot be determined by a single measurement. Therefore, in order to obtain the source magnification, a series of measurements must be made to record the observed flux over time. A plot of the observed flux over time gives a lightcurve.

For any configuration of lenses, the positions of the generated images can be used to calculate the area of the images and the magnification, for any given source position. The simplest method to calculate the source magnification is called the point source approximation, where the amplitude is approximated in the limit where the source and images are shrunk to points of zero size. In this limit, the image magnifications can be calculated analytically from the image positions as shown in this section. In a single lens case, these image positions can be calculated analytically, so a formula describing the magnification for any given source position can be obtained.

Although the point source approximation is simple and efficient, it is limited, and often returns the incorrect source magnification in some cases. Therefore, other methods must be used to calculate the source magnification. Some of these other methods are given in the following chapter. 


\subsubsection{Single Lens Magnification}

In order to obtain the magnification of a source for a single lens configuration, we need to calculate the area of the source as well as the area of the two images produced in a single lens event. If the lens is positioned at the origin, the lens plane is radially symmetric about the origin. This symmetry can be exploited by representing the source and image positions in terms of polar coordinates. The source and the two images can be approximated as sectors of annuli centred at the lens, with a common central angle $\psi$, with widths $\delta w$ and $\delta z_{ \pm}$respectively, and with inner radii $s$ and $z_{ \pm}$respectively.

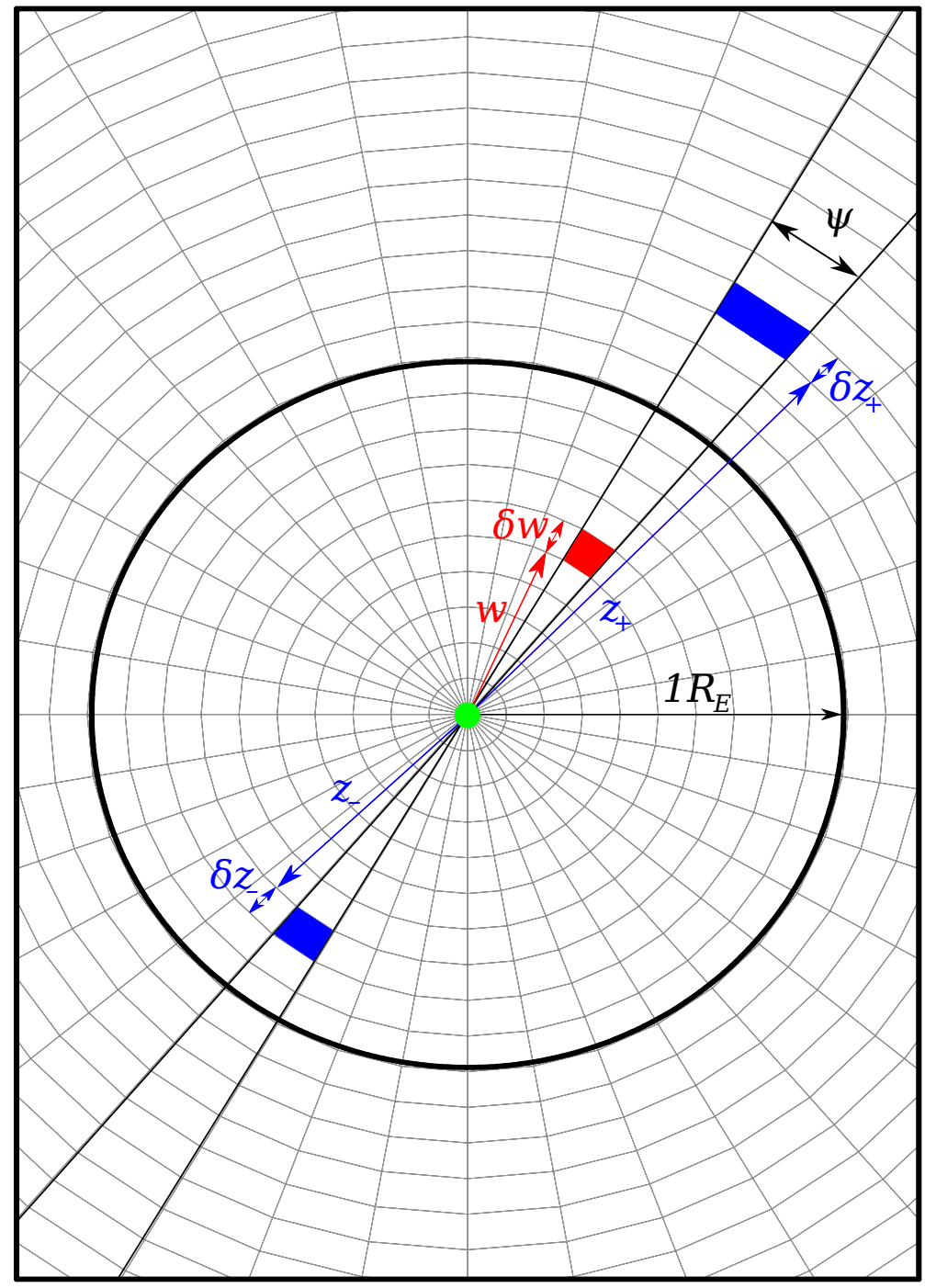

Figure 3.1: A single point lens configuration with the source (red) and images (blue) approximated as sectors of annuli. These sectors can be used to estimate the image magnification. The lens (green) is at the centre and the Einstein ring is shown by bold black circle. 
Figure 3.1 shows the shape and position of the source and images for this arrangement. If the values for $\psi, \delta w$ and $\delta z$ are small, it can be shown that the source and the two images have areas approximately given as $\psi w \delta w, \psi z_{ \pm} \delta z_{ \pm}$ respectively. Therefore, magnification $\mu$ of an image in this diagram can be expressed as,

$$
\mu_{ \pm}=\frac{z_{ \pm} \delta z_{ \pm}}{w \delta w_{ \pm}}
$$

If the areas if the source and images all are shrunk to a point, then the small widths $\delta w$ and $\delta z_{ \pm}$become infinitesimally small, and the quotient of these can be expressed as the derivative $d z_{ \pm} / d w_{ \pm}$. In a single lens case, the image positions can be found using Equation 2.12. This analytical expression gives a negative value for $z_{-}$, which results in a negative value for the area of this image. Therefore, we define the image magnification as the absolute value of the ratio of the areas, which gives us,

$$
\mu_{ \pm}=\left|\frac{1}{2} \pm \frac{w^{2}+2}{2 w \sqrt{w^{2}+4}}\right|
$$

The magnification of the source is defined as the sum of the image magnifications, which gives,

$$
A_{\text {total }}=\frac{w^{2}+2}{w \sqrt{w^{2}+4}}
$$

This equation can be inverted to give the source position in terms of the total source magnification, such that,

$$
w=\sqrt{2\left(\sqrt{\frac{1}{A^{2}-1}+1}-1\right)} .
$$

As discussed earlier, the source magnification is defined as the quotient of the apparent brightness of the source star over the brightness of the source star when unaffected by the lens. Since the brightness of the star when unaffected by the lens is hard to determine from a single microlensing observation, a series of measurements of the apparent brightness is required as the source star moves 
behind the lens. The plot of this apparent brightness over time is referred to as a lightcurve.

Suppose the source star moves in a straight path, with the source moving uniformly in the $\mathrm{u}$-direction, and offset by a constant distance $w_{0}$ in the $\mathrm{v}$-direction. If the source travels an Einstein radius $\left(1 R_{E}\right)$ in time $t_{E}$, and crosses the v-axis at time $t_{0}$, then the relative distance of the source from the lens $w(t)$ can be given by,

$$
w^{2}(t)=w_{0}^{2}+\left[\frac{t-t_{0}}{t_{E}}\right]^{2} .
$$

Assuming that there is no nonlinear movement of the observer, lens, or source star, a lightcurve for a point source and single lens can be analytically determined using just three parameters: the impact parameter $w_{0}$, the Einstein time $t_{E}$, and the crossing time $t_{0}$. If the observer, lens, and source are moving in straight paths but at different velocities, the source will still appear to move in a straight line relative to the lens due to the principle of relativity, and this relative motion can still be expressed in terms of the impact parameter, the Einstein time, and the crossing time.

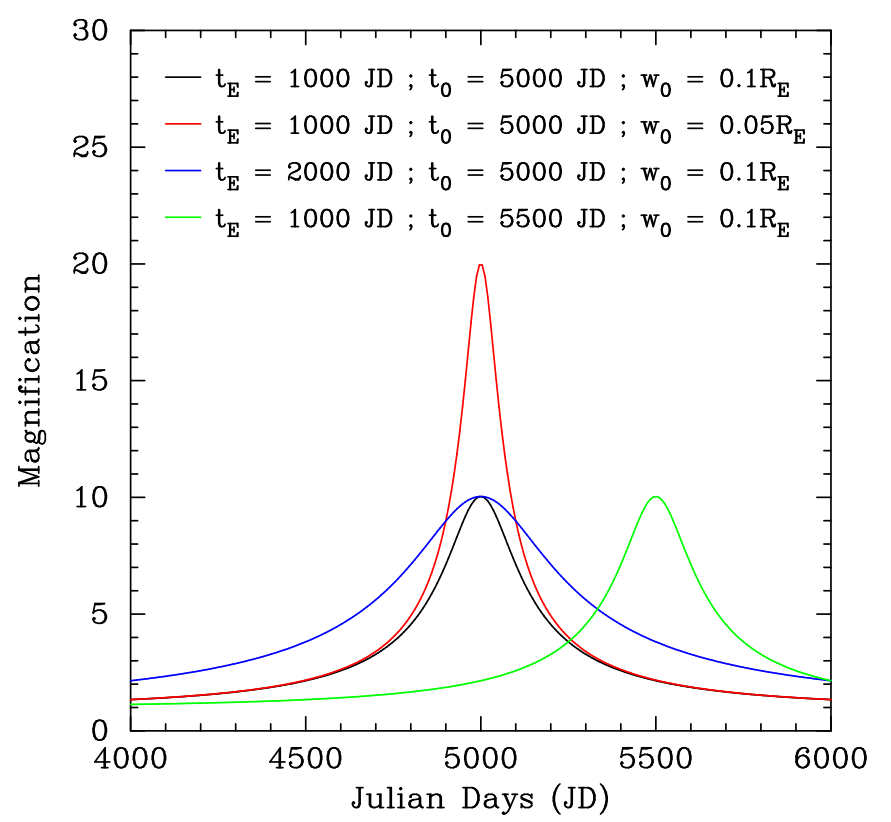

Figure 3.2: Lightcurves that represent the magnification of the source star with time, for 4 microlensing events with various parameters. The Einstein crossing time, $t_{E}$, peak time, $t_{0}$ and impact parameter, $w_{0}$, affects the width, peak position and peak height of the lightcurve respectively. 
Figure 3.2 shows several plots of the magnification of a source star against time as it moves behind the single lensing mass. All of the plots give a bell like curve not dissimilar to a resonance curve, but the height, width, and position of each curve varies depending on the values for the impact parameter, the Einstein time, and the crossing time respectively. From these plots, it can be seen that increasing or decreasing the Einstein time widens or narrows the curve respectively, while increasing or decreasing the crossing time shifts the curve to the right or left respectively.

The height of the curve is governed by the impact parameter. A larger impact parameter gives a shorter peak, while a smaller impact parameter gives a taller peak. For very small impact parameters it can be shown, using Equation 3.3, that the height of the peak for a given impact parameter, $w_{0}$, can be estimated by,

$$
A_{\text {peak }} \approx \frac{1}{w_{0}} .
$$

As the source approaches the point directly behind the lens $\left(w_{0} \rightarrow 0\right)$, the magnification of the point source diverges. This clearly does not happen in real gravitational microlensing events. Therefore, although calculating the point source magnification is easy and efficient, it has limitations where it returns the wrong result. In these cases, other methods must be used to calculate the source magnification, such those introduced in the next chapter.

\subsubsection{Multiple Lens Magnification}

Calculating the magnification of a image produced by a multiple lens system differs from that produced by the single lens case in two ways.

Firstly, when we generalise the point source magnification to a configuration with more than one lens, we lose the radial symmetry of the image plane about the primary lens. Therefore, there is no obvious advantage to use polar coordinates over Cartesian coordinates, as we did in the single lens case.

Secondly, as discussed in Chapter 2, there is no analytical solution to the position of the images for a multiple lens system, so unlike the single lens case, 
there is no analytical solution for the point source magnification in the multiple lens cases.

The lens equation in Equation 2.11 described a mapping from a point on the image plane to a point on the source plane. Vector calculus tells us if an infinitesimal area $\delta x \delta y$ is mapped onto the source plane, its projected area $\delta u \delta v$ can be given by the Jacobian determinant, often called the Jacobian for brevity. The Jacobian gives an infinitesimal area element on the source plane divided by its corresponding area element on the image plane (see Appendix B). Therefore, the point image magnification, $\mu$, which is the ratio of the area of the image to the area of the source, can be described as the inverse of this determinant,

$$
\mu=\frac{1}{J}
$$

and the total magnification, $A$, of the source can be expressed as the sum of all the (physical) image magnifications, $\mu$, such that,

$$
A=\sum_{\text {images }} \mu=\sum_{\text {images }} \frac{1}{J} .
$$

If a position on the image plane is given by $(x, y)$, and its corresponding position on the source plane is given by $(u, v)$, then the Jacobian determinant is given by,

$$
J=\left|\begin{array}{ll}
\frac{\partial u}{\partial x} & \frac{\partial u}{\partial y} \\
\frac{\partial v}{\partial x} & \frac{\partial v}{\partial y}
\end{array}\right|=\frac{\partial u}{\partial x} \frac{\partial v}{\partial y}-\frac{\partial v}{\partial x} \frac{\partial u}{\partial y} .
$$

As shown in Appendix B, this Jacobian is exactly equivalent to,

$$
J=\left|\begin{array}{ll}
\frac{\partial w}{\partial z} & \frac{\partial w}{\partial \bar{z}} \\
\frac{\partial \bar{w}}{\partial z} & \frac{\partial \bar{w}}{\partial \bar{z}}
\end{array}\right|=\frac{\partial w}{\partial z} \frac{\partial \bar{w}}{\partial \bar{z}}-\frac{\partial \bar{w}}{\partial z} \frac{\partial w}{\partial \bar{z}} .
$$

When applying the Jacobian in Equation 3.9 to the lens equation, we get,

$$
J=\left|\begin{array}{cc}
1 & \kappa(z) \\
\kappa(z) & 1
\end{array}\right|=1-|\kappa(z)|^{2}
$$

where $\kappa(z)$ is given by,

$$
\kappa(z)=\frac{\partial \bar{w}}{\partial z}=\sum_{i=1}^{N} \frac{\epsilon_{i}}{\left(z-r_{i}\right)^{2}} .
$$




\subsection{Image Topology}

The lens equation describes a mapping from the image plane with point $z=x+i y$ to the source plane $w=u+i v$. Each image on the image plane corresponds to one unique position on the source plane. However, as shown in Chapter 2, when this mapping is inverted, one point on the source plane corresponds to up to $N^{2}+1$ points on the image plane for a system with $N$ lenses.

To better visualise this mapping from the source plane to the image plane, we can imagine the image plane as a deformable and stretchable sheet called a 'sky sheet' (Mollerach and Roulet, 2002). In this picture, the lens equation describes the deformation of the sky sheet, including how the sheet stretches and where it folds over itself.
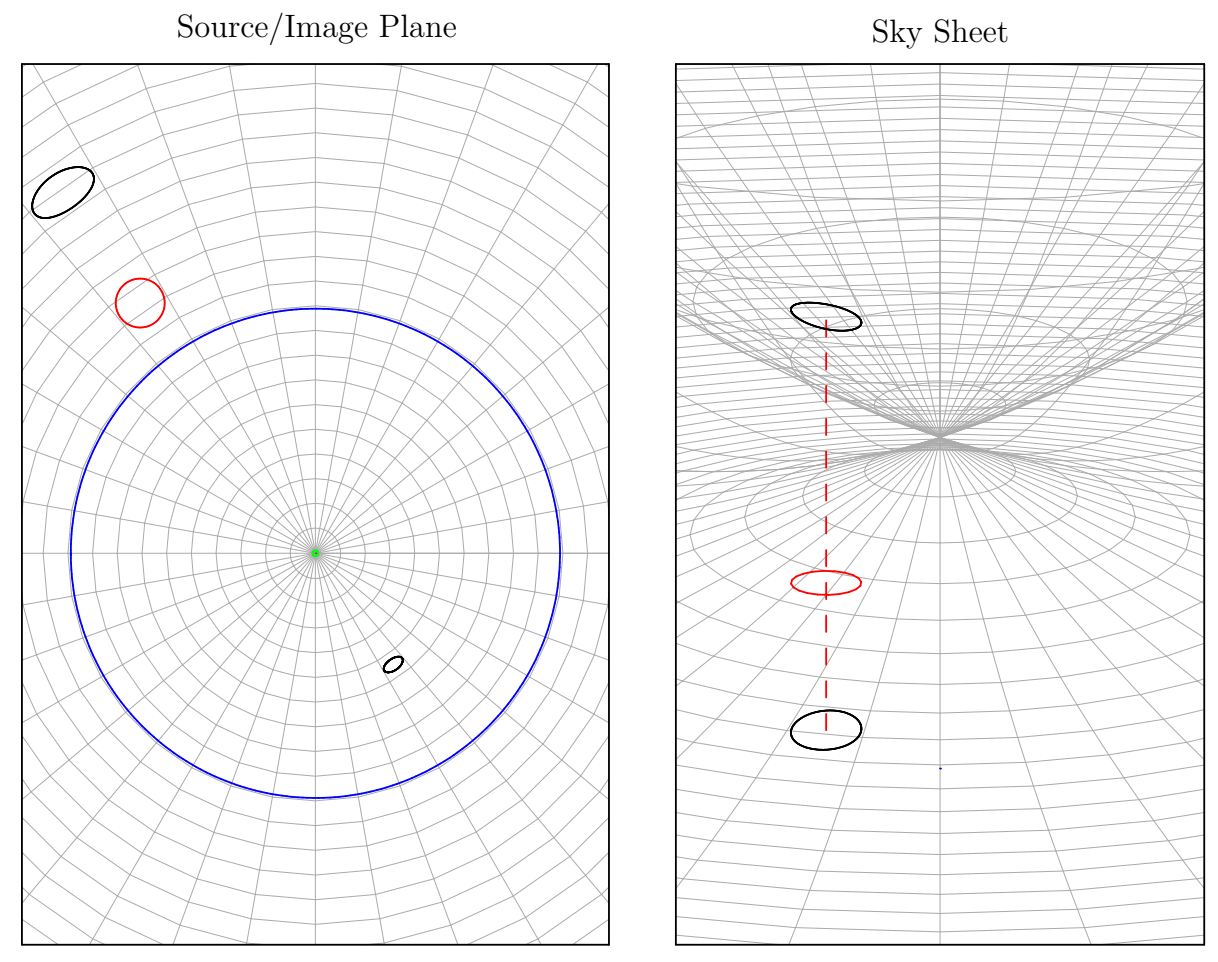

Figure 3.3: The sky sheet and source/image plane for a single lens, with the Einstein ring is represented by the blue ring in the source/image plane. This sky sheet demonstrates how the source is projected vertically up and down to produce images inside and outside the Einstein ring.

The right panel of Figure 3.3 shows the sky sheet deformation for a single lens, while the left panel shows the position of the source and images on the lens plane. Since the single lens has circular symmetry about the lens, the positions 
on the image plane are represented by polar coordinates. The two horizontal axes of the 'sky space' represent the $u$ and $v$ coordinates on the source plane, and the vertical height represents the negative of the Jacobian of the image. The lower part of the sky sheet represents the region on the image plane outside the Einstein ring, whereas the upper part of the sky sheet represents the region on the image plane inside the Einstein ring. It should be noted that the upper part of the sky sheet is upside down, so that the projected image of the source on this part of the sky sheet is inverted. Therefore, the image on the upper part of the sky sheet would have a negative parity, while the image on the lower part would have a positive parity.

The sheet crossing over itself at $(u, v)=(0,0)$ with a height of 1 , represents the way the images form the Einstein ring (with a radius of 1) when the source is directly behind the lens. Figure 3.4 shows the lens plane and the sky sheet in the situation where the Einstein ring is formed.
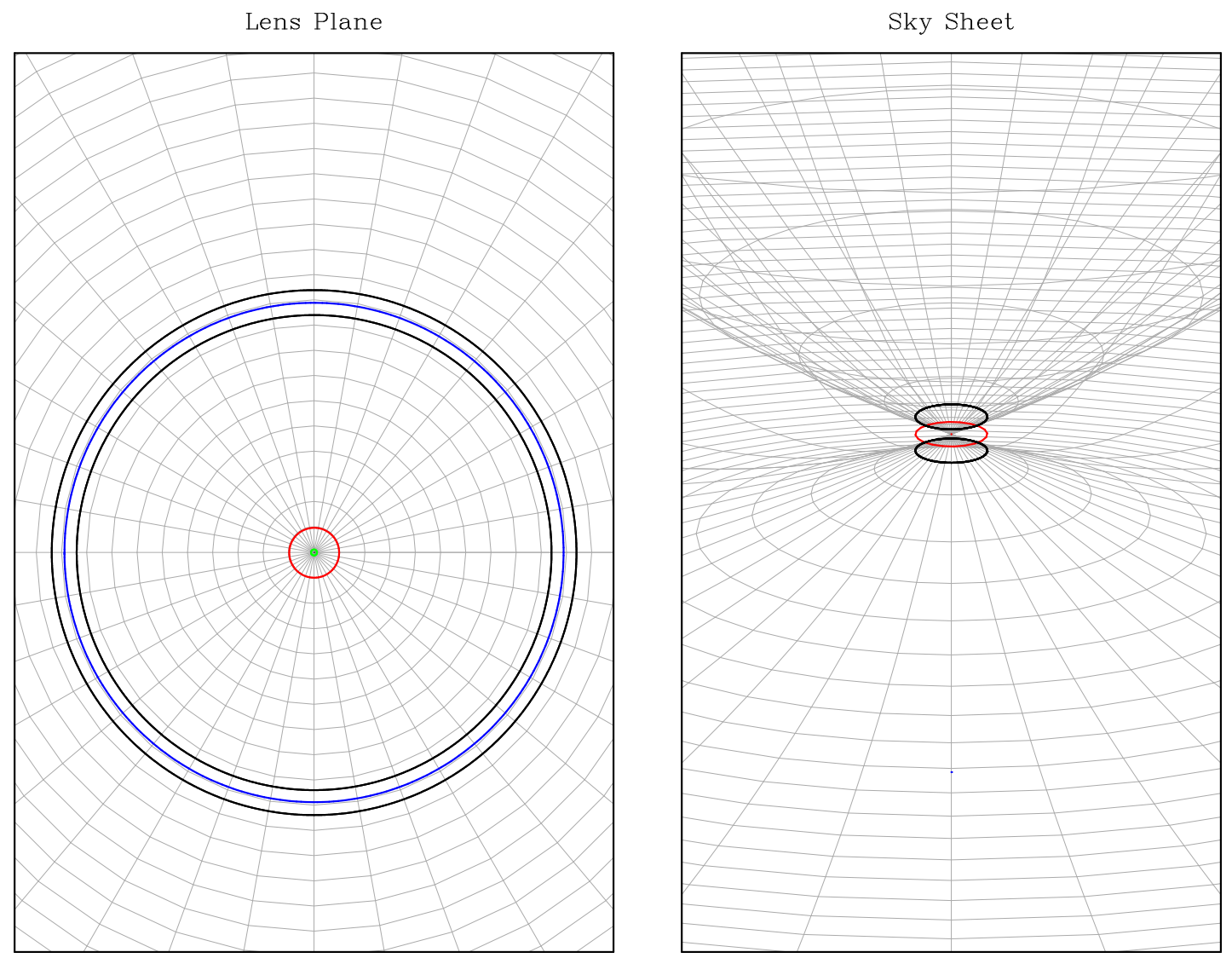

Figure 3.4: Sky sheet and source/image plane for a single lens for the formation of the Einstein ring. This sky sheet demonstrates how a source at the lens position is projected vertically up and down to produce the Einstein ring. 
When determining the magnification of an image from a sky sheet, it can be shown that the magnification of an image depends on two properties: the slope of the sky sheet at a particular source position, and the amount that the sky sheet is stretched at that position. When the source is projected onto part of the sky sheet with a steeper gradient, the image is spread over a larger area, which would give a larger image magnification if the sky sheet is not stretched here. However, some parts of the sky sheet are stretched, so when the source is projected onto these parts of the sky sheet, the corresponding area on the image plane is smaller. This is exemplified in the upper parts of the sky sheet, which correspond to the regions on the image plane close to the lenses. Whereas on the lower parts of the sky sheet, the sky sheet is close to flat and has been stretched very little, so the total magnification of the image here is close to one.
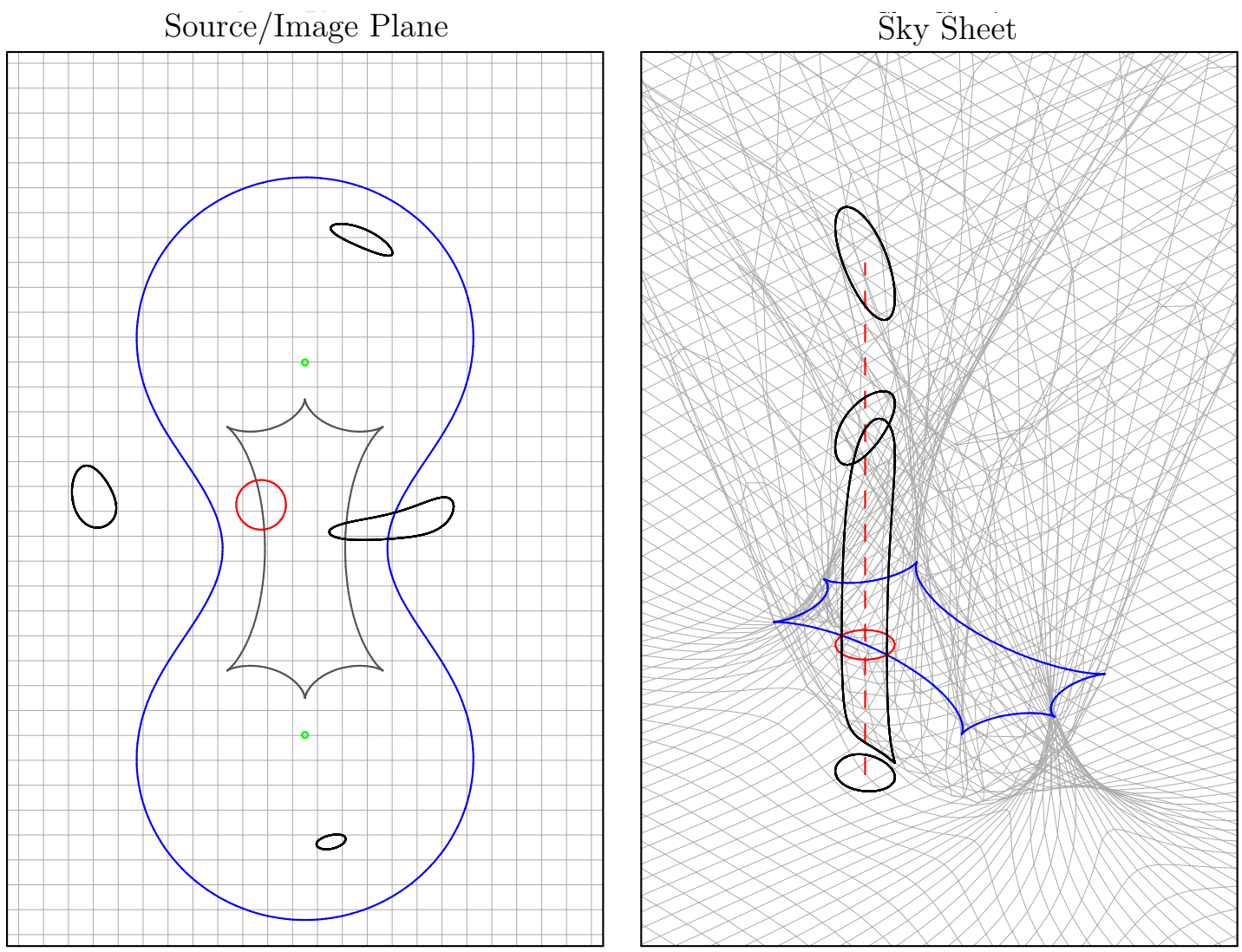

Figure 3.5: Sky sheet and source/image plane for a binary lens system, showing critical curves in blue and caustic curves in grey in the latter. This sky sheet demonstrates how a source crossing the caustic curve is projected to form one image outside the critical curves, two images inside the critical curves, and two part images joining at the critical curves.

The left panel of Figure 3.5 shows the lens plane for a binary lens configura- 
tion, and the right panel of this figure gives the sky sheet for this configuration. This is clearly more complex that the 'simple' sky sheet of a single lens, as the sky sheet in this binary lens case folds over itself several times. The blue lines on the sky sheet represent the places where the sheet is folded. If we were to take the sky sheet and unfold it to make the image plane, these blue fold lines become the critical curves on the image plane. The critical curves can be considered as generalisations of the Einstein ring - the boundary between images of positive and negative parity. The places where the folds in the sky sheet are projected onto the source plane are called the caustic curve folds, and where two caustic folds meet is called a caustic cusp. These are represented by the smooth grey curves and the sharp corners on the source plane respectively.

The sky sheet is folded is such a way that there are three layers of sky sheet above the region outside the caustic curves, while there are five layers above the region inside the caustic curve. Therefore, when the source is outside the caustic curve, three images are formed (one with positive parity and two with negative parity), while five images form when the source is inside the caustic curve (two with positive parity and three with negative parity).

Figure 3.5 shows a case where the source straddles a caustic curve. When this source is projected onto the sky sheet, an image is created along a fold in the sky sheet. This corresponds to an image along the critical curve on the image plane. However, this image is actually made from two 'part' images connected at the critical curve. This part of the sky sheet is only over part of the source, as it is only the source inside the caustic curve that is projected onto this part of the sky sheet.

In a configuration with $N$ lenses, when the source is outside the caustic folds, there is always one image with positive parity located near the source, and $N$ images with negative parity, each located near one of the lens positions. Therefore, the minimum number of images is $N+1$. This result was first shown mathematically by Witt (1990). Whenever the source crosses a caustic curve, one positive and one negative image is created. Therefore a system with $N$ lenses always has a total parity of $1-N$. 
Since the number of images is determined by the position of the source, it is useful to divide the source plane into 'caustic domains' [Rhie 2001]. In this notation, $\mathcal{D}^{0}$ is the caustic domain outside the caustic curves where $N+1$ images are produced, $\mathcal{D}^{1}$ is the caustic domain region where $N+3$ images are produced, and so on. Higher domains such as $\mathcal{D}^{2}$ are created when the sky sheet is folded over again to create a set of caustic folds inside another set of caustic folds. In general, $N+1+2 n$ images are produced whenever the source is in the caustic domain $\mathcal{D}^{n}$. Figure 3.6 below shows the caustic curves, critical curves and domains triple lens system.
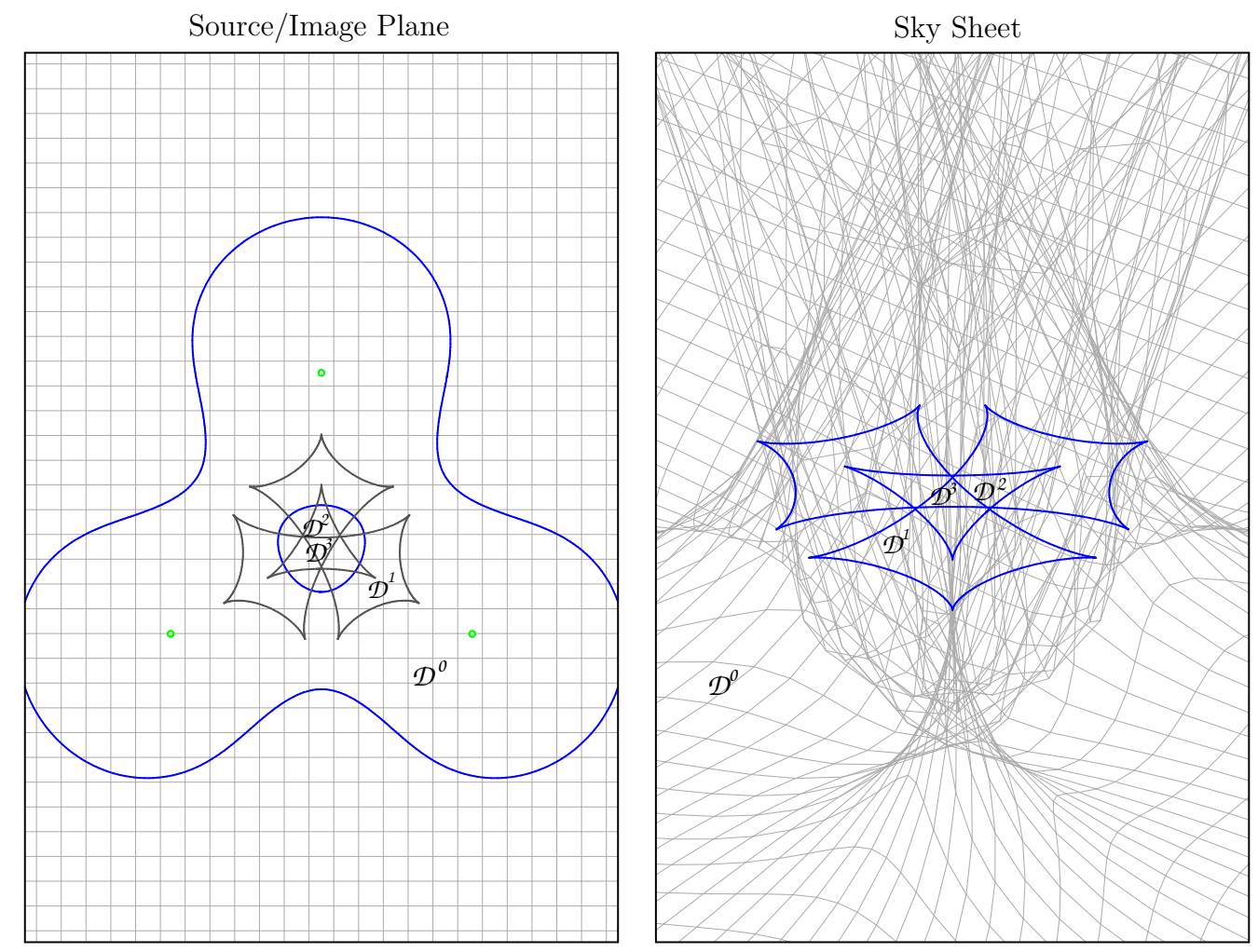

Figure 3.6: Sky sheet and source/image plane for a triple lens system, showing caustic domains $\mathcal{D}^{0}, \mathcal{D}^{1}, \mathcal{D}^{2}$ and $\mathcal{D}^{3}$. This sky sheet demonstrates how the sheet folds over itself to produce caustic curves on the source plane. The places where the sky sheet folds over correspond to critical curves on the image plane. Multiple domains are formed as the sky sheet folds over itself multiple times.

\subsection{Critical and Caustic Curves}

From Figure 3.5, it can be seen that the tangent of the sky sheet on the edge of a fold is vertical, giving an infinite gradient. Since the sky sheet is not infinitely 
stretched at these folds, an infinite gradient gives an infinite magnification whenever a point source is projected onto the edges of these folds. Therefore, the critical curves can be given as the points on the image plane where the point image magnification is infinite. The caustic curves are then given by the corresponding positions on the source plane. As shown in Equation 3.6, an infinite point image magnification corresponds to a zero Jacobian. Therefore, it can be shown from Equation 3.10 that the critical curves are the loci of points on the image plane that are given by,

$$
\kappa(z)=e^{i \phi}
$$

where $\phi$ is any angle between 0 and $2 \pi$. Additionally, from Equation 3.11 it can be shown that,

$$
\kappa=\frac{\partial \bar{w}}{\partial z}=\frac{\partial}{\partial z}\left[\frac{G(z)}{H(z)}\right] .
$$

Therefore, it can be shown that the critical curves $z$ can be given by the polynomial,

$$
0=G^{\prime} H-H^{\prime} G-H^{2} e^{i \phi}
$$

where $G$ and $H$ are the same polynomials given in Equations 2.17 and 2.18, and $G^{\prime}$ and $H^{\prime}$ are their derivatives respectively. By obtaining the expressions for the coefficients of $G$ and $H$ (see Appendix C), the critical curves can be given by the solutions of the polynomial,

$$
0=\sum_{n=0}^{2 N} c_{n} z^{n}
$$

where the coefficients can be given by,

$$
c_{n}=\sum_{k=0}^{n}\left[(k+1)\left(G_{k+1} H_{n-k}-G_{n-k} H_{k+1}\right)-H_{k} H_{n-k} e^{i \phi}\right] .
$$

For a system with $N$ lensing bodies, the polynomial given in Equation 3.15 has $2 N$ roots for any value of $\phi$. Values for $\phi$ in the range between 0 and $2 \pi$ can be substituted into this polynomial, which can then be solved for each value of $\phi$ using the numerical root-finding algorithms, to give points along the critical curves. Two numerical root-finding algorithms, the Laguerre and Jenkins-Traub 
algorithms are discussed in Chapter 5. Once the critical curve positions have been found, these $z=x+i y$ points can be substituted into the lens equation to give the corresponding point on the source plane to describe the caustic curves.

In a single lens case where the lens is located at the origin, it can be shown that the points on the critical curves are given by the relationship $z=e^{i \phi}$, whose solutions describe the unit circle - the Einstein ring.

\subsection{Binary Lens}

The simplest case with a caustic structure is the binary lens system. While the caustic curves in higher lens systems often intersect and form a caustic structure with multiple domains, the caustic curves in a binary structure do not. Thus domain $\mathcal{D}^{1}$ is formed inside the caustic structure and $\mathcal{D}^{0}$ outside. However, by investigating the nature of the binary caustic structure, we can develop an understanding of caustic curves, which can then be applied to higher lens systems.

As in section 1.1 above, a simple single lens event has three parameters. However, a multiple lens systems has three extra parameters for each lens added to the system. If the primary lens is located at the origin, these extra parameters are the mass ratio or mass fraction for each lens, the $\mathrm{x}$ and $\mathrm{y}$ positions or the modulus, and the argument for each lens. If the primary lens is much larger than the other lenses, then it is likely that the primary lens is a star (or black hole) with planets orbiting it.

A binary lens event can be classified as a close binary, a far binary, or an intermediate binary, as shown in Figures 3.7 and 3.8 below.

\subsubsection{Caustic and Critical Curves}

The shape and structure of the critical and caustic curves for a binary lens ultimately depend on only two parameters: the distance between the lenses $d$, and the mass ratio q. Figure 3.7 and Figure 3.8 show the how the structure of the critical and caustic curves change as the lens separations increases for binary lenses with mass ratios $q=1$ and $q=0.1$ respectively. The critical and caustic curves are represented as red and blue lines respectively, and the scales on the 
axes are given in terms of units of Einstein radii.

\section{Close Binary Lens}

Let us first consider the case where the two lenses are initially at the origin. In this situation, the critical curves will form the Einstein ring and the caustic curves form a single point at the origin. As the two lenses move apart, the critical curve becomes less circular, and the caustic curve grows into a 4-cusp caustic loop, which hereafter will be called the central caustic. If the two lenses have equal mass, then this central caustic grows into a symmetrical diamondlike shape exactly midway between the lenses. However, if the masses of the two lenses are unequal, this caustic curve grows into a kite-like shape, and is positioned closer to the larger lens. As the lens separation continues to grow, two smaller 3-cusp caustic loops (hereafter called planetary caustics) approach the binary lens from infinity. When the masses are equal, these planetary caustics approach at the direction perpendicular to the lens axis, and join with the central caustic when the lens separation is $d=1 / \sqrt{2} \approx 0.707$. However, when the mass ratio is unequal, the planetary caustics approach from lines tilted toward the larger (primary) mass side of the binary, and as the mass ratio becomes more unequal, these planetary caustics will approach along lines closer to the lens axis (Han, 2006).

Two small critical curve loops corresponding to these planetary caustic curves grow inside the continually distorting Einstein ring and move to join up with this larger critical curve structure. It should be noted that the images which appear inside these smaller critical loops have positive parity. When the masses are equal, these critical loops appear and move in a line equidistant from the two lenses, and join with the larger critical loop when the lens separation is $d=\frac{1}{\sqrt{2}}$. However, when the masses are unequal, these small critical loops appear closer to the smaller lens and move towards the 'planetary' side of the binary lens. When the caustic curves and critical curves join to form a single caustic curve and critical curve structure respectively, the binary lens then becomes known as an intermediate binary. 


\section{Intermediate Binary Lens}

A binary lens is considered to be an intermediate binary when the separate critical curve loops have joined to become a single critical curve surrounding the lenses, and the caustic curves have combined to form a single 6-cusp caustic structure called a resonant caustic. When the lens separation is $d=1$, the caustic structure will always form a resonant caustic regardless of the mass ratio. As the separation increases, the caustic and critical curves continue to distort, until the resonant caustic splits into two smaller caustic loops. This occurs when the lens separation is $d=2$ if the two masses are equal.

\section{Wide Binary Lens}

Two lenses are considered to be a wide binary when the caustic curve has split into two smaller 4-cusp caustic structures, and the critical curve loop has split into two smaller loops, surrounding the two lenses. If masses are unequal, then the caustic structure closer to the larger mass is called the central caustic, while the caustic structure closer to the smaller mass is called the planetary caustic. The central caustic has a much smaller area than the planetary caustic, while the planetary caustic is shaped more diamond-like in shape, and is increasingly more diamond-like for increasingly unequal masses.

As the separation distance gets larger, the critical curves become more circular. As $d \rightarrow \infty$, the caustic curves become points at the lens positions, and the critical curves become the circular, or Einstein rings with a radius $\sqrt{\epsilon_{j}}$ for each lens. Therefore the binary lens resembles two single lenses in this limiting case.

There are two cases where the resonant caustic splits into a planetary caustic and central caustic: when a close binary becomes an intermediate binary, at the lens separation $d_{I}$, and when an intermediate binary becomes a wide binary, at the lens separation $d_{I I}$.

When the masses are equal $(q=1)$, the values of these lens separations are $d_{I}=\frac{1}{\sqrt{2}}$ and $d_{I I}=2$, as mentioned earlier. Erdl and Schneider (1993) showed that, if the mass fractions of the primary and planetary lenses are $\epsilon_{1}=1 /(1+q)$ and $\epsilon_{2}=q /(1+q)$ respectively, the expressions for the lens separations $d_{I}$ and 


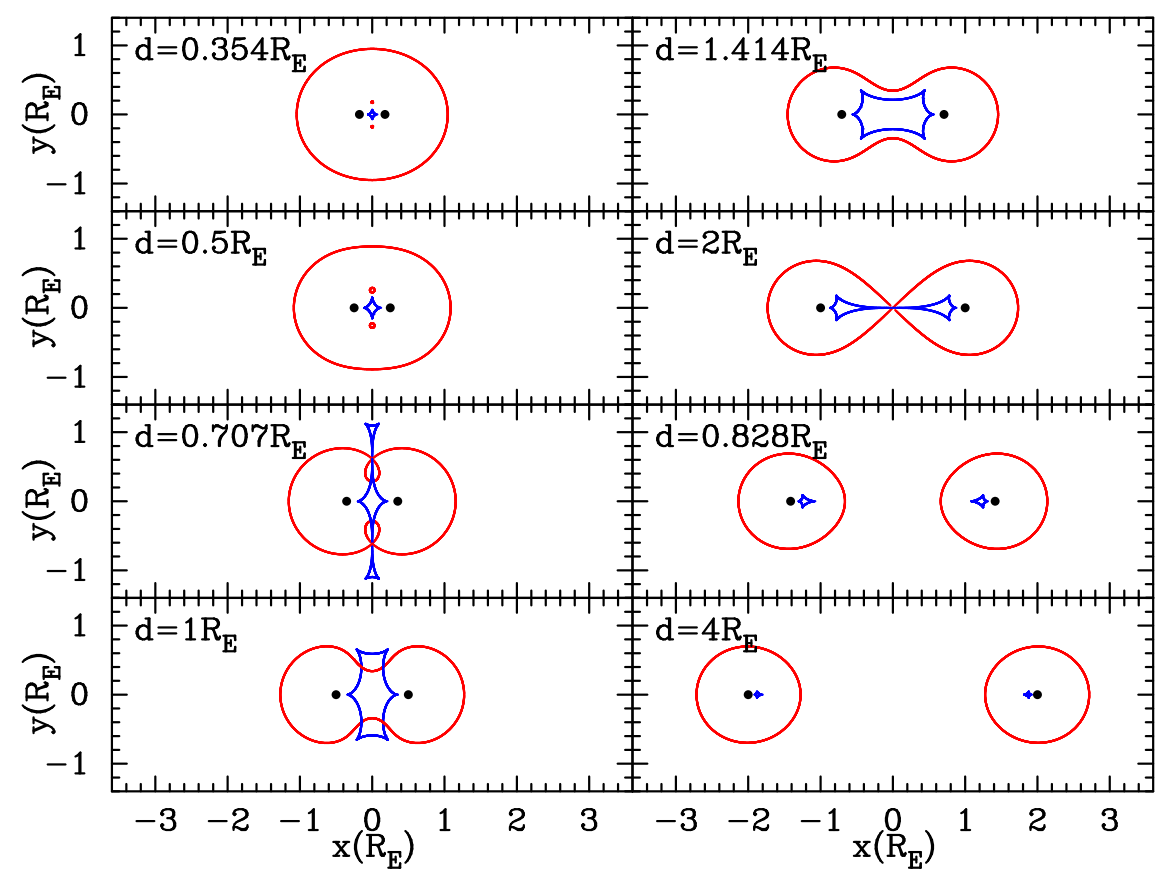

Figure 3.7: Critical curves (red) and caustic curves (blue) for a binary lens system with $q=1$ at various lens separations.

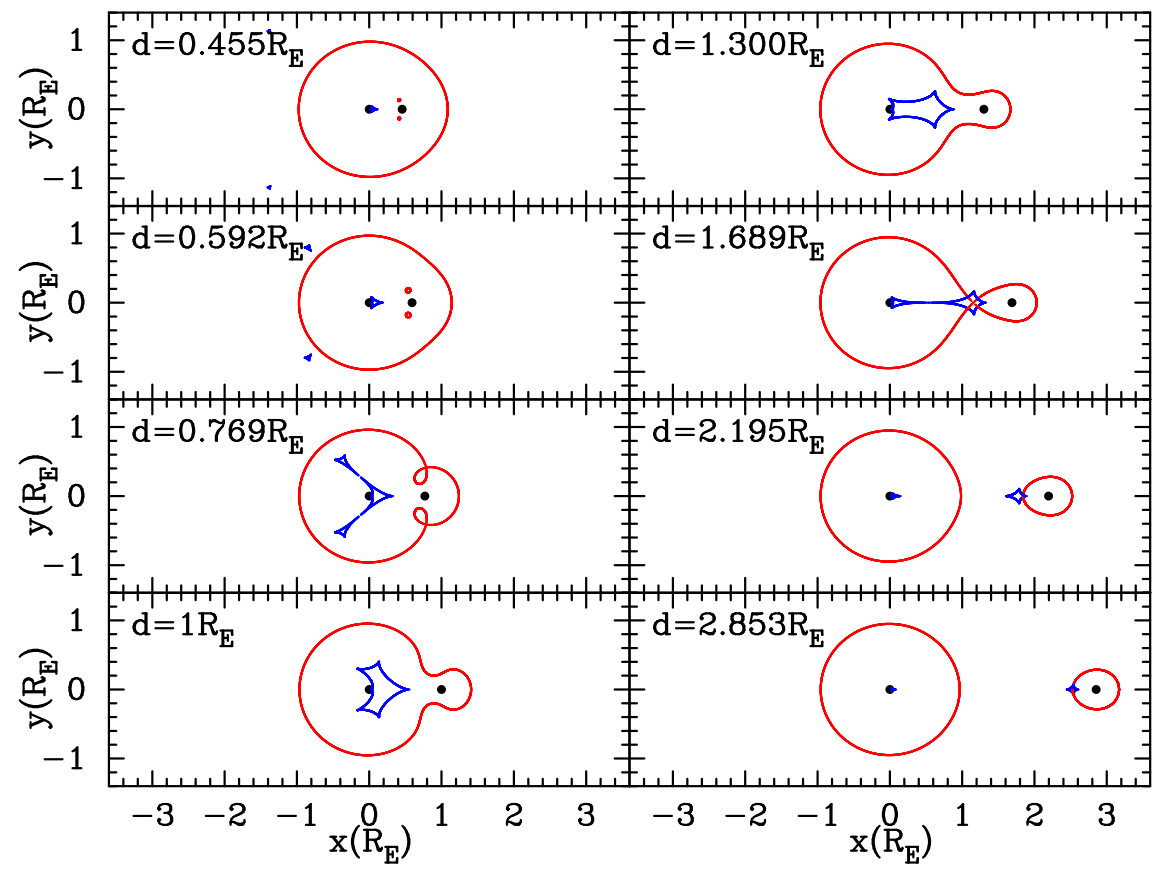

Figure 3.8: Critical curves (red) and caustic curves (blue) for a binary lens system with $q=0.1$ at various lens separations. 
$d_{I I}$ can be given by,

$$
\begin{aligned}
\epsilon_{1} \epsilon_{2} & =\frac{1}{d_{I}^{8}}\left(\frac{1-d_{I}^{4}}{3}\right)^{3}, \\
\left(\epsilon_{1}^{1 / 3}+\epsilon_{2}^{1 / 3}\right)^{3} & =d_{I I}^{2} .
\end{aligned}
$$

It can be shown that $d_{I I}=1 / d_{I}^{2}$ for all mass ratios. Equations 3.17 and 3.18 can be rearranged to give the lens separations $d_{I}$ and $d_{I I}$ in terms of the mass ratio $q$, such that,

$$
\begin{aligned}
d_{I} & =\sqrt[4]{\frac{1+q}{\left(1+q^{1 / 3}\right)^{3}}}, \\
d_{I I} & =\sqrt{\frac{\left(1+q^{1 / 3}\right)^{3}}{1+q}},
\end{aligned}
$$

Figure 3.9 show the range of parameters that determine whether a binary lens is a close binary, an intermediate binary, or a wide binary. The lens separations $d_{I}$ and $d_{I I}$ are shown on this graph as lines between these cases. This graph shows that in the limit where $q \rightarrow 1$ the lens separations become $d_{I} \rightarrow \frac{1}{\sqrt{2}}$ and $d \rightarrow 2$. As $q \rightarrow 0$, both these lens separations become $d_{I}, d_{I I} \rightarrow 1$, showing that the caustic structure always forms a resonant caustic when $d=1$, regardless of the mass ratio.

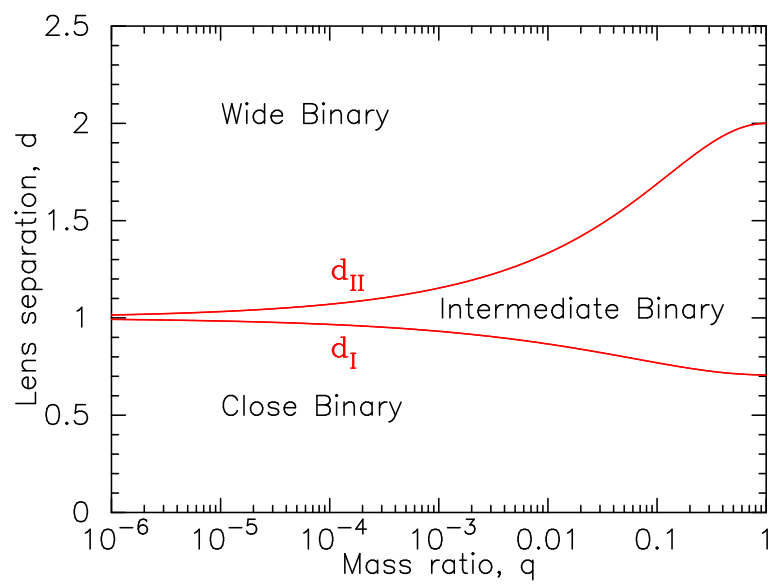

Figure 3.9: The close, intermediate and wide binary cases in parameter space, with the boundaries $d_{I}$ and $d_{I I}$ between each case. 


\section{Caustic Curves in Planetary Configurations}

A planetary system can be defined as a system where $q<<1$. In a binary lens system like this, we can define the 'front' of the binary as the same side with the planetary lens, and the 'back' of the binary as the opposite side. When a system such as this has a lens separation $d<1$, part of the caustic curve will usually be 'behind' the primary lens.

As the mass ratio decreases for a binary lens, the main critical curve will get larger, approaching the Einstein ring as $q \rightarrow 0$. In a wide binary, this is accompanied by the critical loop surrounding the planetary lens getting smaller, while in a close binary, the two smaller critical curves not only become small, but also move towards the planetary lens. In the planetary limit where $q \rightarrow 0$, the distance between the primary lens and the planetary caustic approaches $d-1 / d$ (Han, 2006), where a positive value represents the 'front' of the binary lens, and a negative value represents the 'back'. We can easily invert this equation to give the lens separation, $d$, in terms of the planetary caustic position, $w$, such that,

$$
d=\frac{w+\sqrt{w^{2}+4}}{2}
$$

In the complex coordinate representation, where $r_{j}$ is the complex number representing the position of the $j$ th lens, the approximate position of the planetary caustics $w$ from the origin can be given by,

$$
w=r_{2}-\frac{1}{r_{2}-r_{1}}
$$

This result comes directly from the lens equation by taking $z \approx r$ as the position of the planetary critical curves in the limit $\epsilon_{1} \rightarrow 1, \epsilon_{2} \rightarrow 0$. Note that when the lens separation is $d=\sqrt{2}$, the midpoint between the two lenses, $1 / \sqrt{2}$ from either lens, is always within the planetary (or resonant) caustic.

\subsubsection{Point Source Magnification}

In contrast to the single lens case, where the point source magnification diverges at the lens, the point source magnification in a binary lens event only diverges 
at the caustic curves. In this binary lens case, the caustic curves never touch the lenses, however, the caustic curves approach the both lenses in the limits $d \rightarrow 0$ and $d \rightarrow \infty$. Only in these limits, will the point source magnification appear to diverge at two lens positions.

When a binary lens configuration has a large mass ratio $q<<1$, the central (or resonant) caustic is very small and is located close to the primary lens. In this configuration, the overall shape of the lightcurve can be expressed as a single lens light curve with some 'residual' deviations, as demonstrated in Figures 3.11 below. In these situations, a majority of the data can be modelled using the single lens analysis, to find the approximate parameters for the impact parameter, Einstein time and crossing time. The nature of the binary lens itself can then be analysed by looking at the 'residuals'.

The features in a lightcurve caused by the interactions with the critical curves can be divided into two types: the feature from the point source's interaction with the caustic folds, and the features from its interaction with the caustic cusps. Figure 3.10 shows the magnification map for a binary lens with a lens separation $d=0.8 R_{E}$ and a mass ratio of $q=0.04$, with three source tracks (a), (b) and (c) crossing the (black) caustic curves. Figure 3.11 shows the resultant lightcurves for these three source tracks respectively.

As mentioned earlier in this chapter, the point source magnification suddenly diverges when the source star hits a caustic fold, and the lightcurve forms a Ushape as the point source travels from one side of the caustic structure to the other. This is shown in source track (a) in Figures 3.10 and 3.11. When the point source enters (or exits) the caustic structure via a caustic cusp, the source magnification rises (or falls) more slowly as it approaches (or goes away from) the cusp. Gaudi and Petters (2002) showed that, if $w_{c}$ is the distance between the cusp and the point source, the source magnification rises (or falls) as $A \propto w_{c}^{-1}$ when the source approaches (or goes away from) the cusp along the 'axis' of the cusp (shown in source track (c) in Figures 3.10 and 3.11), but rises as $A \propto w_{c}^{-2 / 3}$ when the source approaches perpendicular to this axis.

When the point source passes near a cusp, the source magnification does not 
diverge as it would when hits a caustic curve, but it does rise as the source passes the cusp, reaching a maximum when it is positioned along the cusp 'axis'. This is demonstrated by source track (b) in Figures 3.10 and 3.11 .

Figures 3.10 and 3.11 also show the low magnification trough that forms whenever a planetary binary lens has a lens separation $d<1$. This is the region between the caustic curves 'behind' the primary lens where the magnification of the source is lower than the corresponding single lens source magnification.

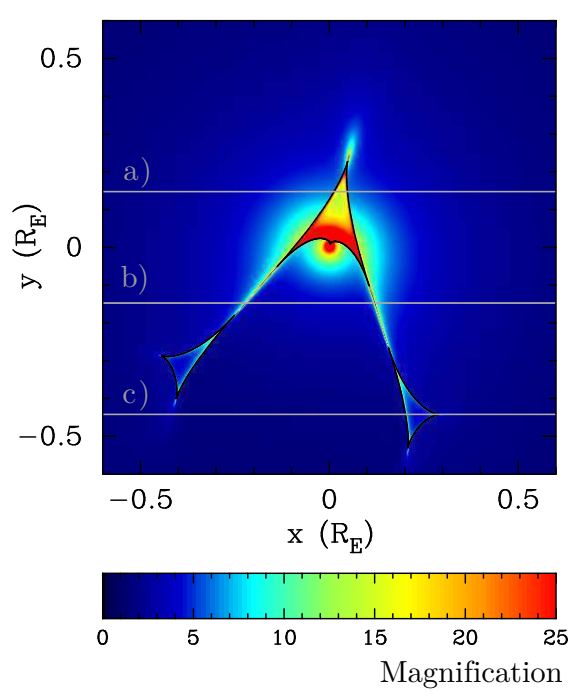

Figure 3.10: Magnification map for a binary lens event showing three source tracks a, b and c.

\subsubsection{Image Positions and Magnifications}

To study the magnification of a point source in more details, we can look at the magnification of each image, and determine the contribution each image has on the total source magnification.

Figure 3.12 presents the source plane for the binary lens configuration shown in Figure 3.10, with source track (c). Figure 3.13 presents the image plane for this binary lens configuration, with Figure 3.14 showing a close up of the image plane in the vicinity of the planetary lens. The grey lines and arrows represent the paths of the unphysical roots, while the coloured lines and arrows represent the paths of the (physical) images. Figure 3.15 shows the lightcurve of the event in 


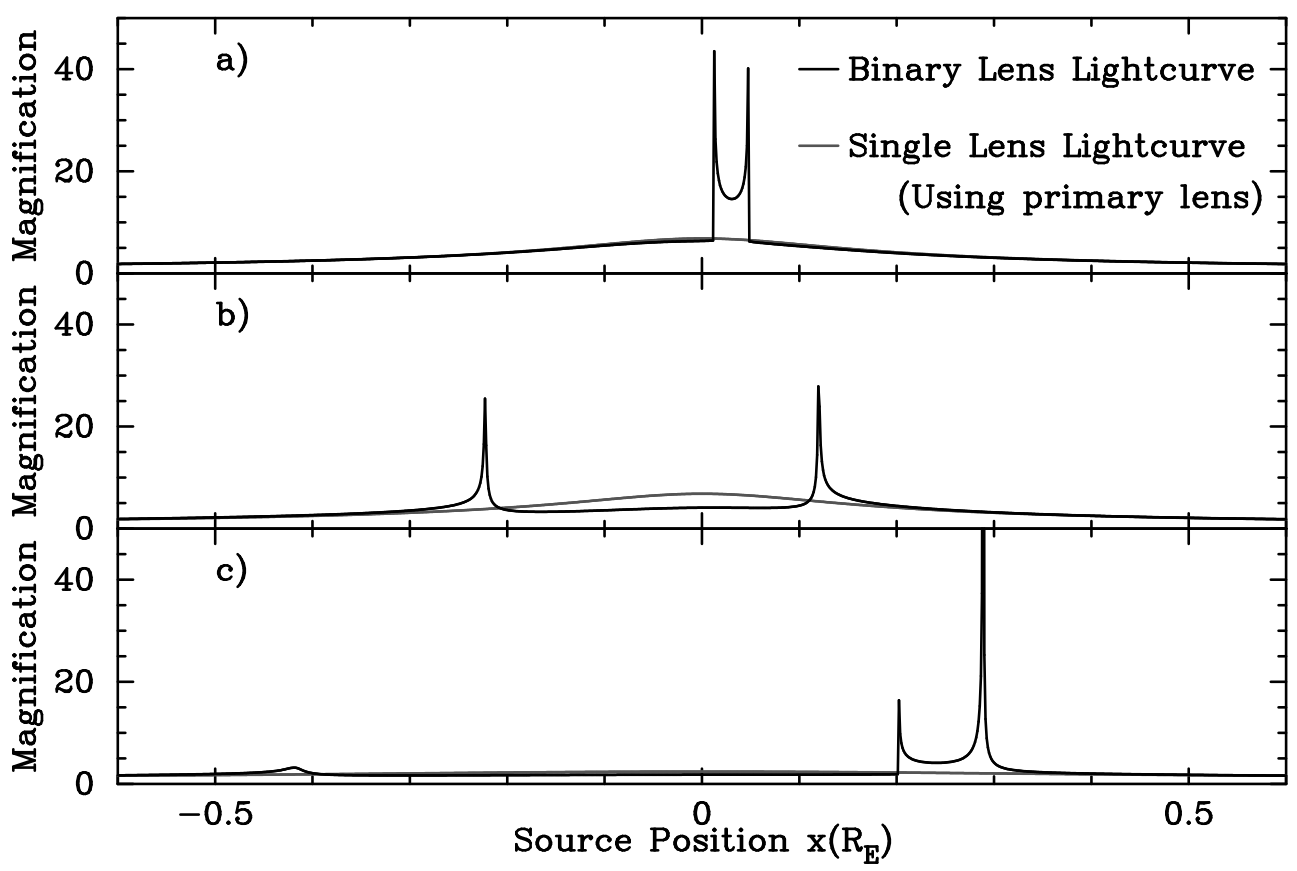

Figure 3.11: Lightcurves for a binary lens event for three source tracks a, b and c in Figure 3.10 .

black, and the magnification of each (physical) image in its respective colour. The green arrow head shows the position on the lightcurve and the source track where the source passes a caustic cusp, along with the image position that corresponds to this rise in the image magnification.

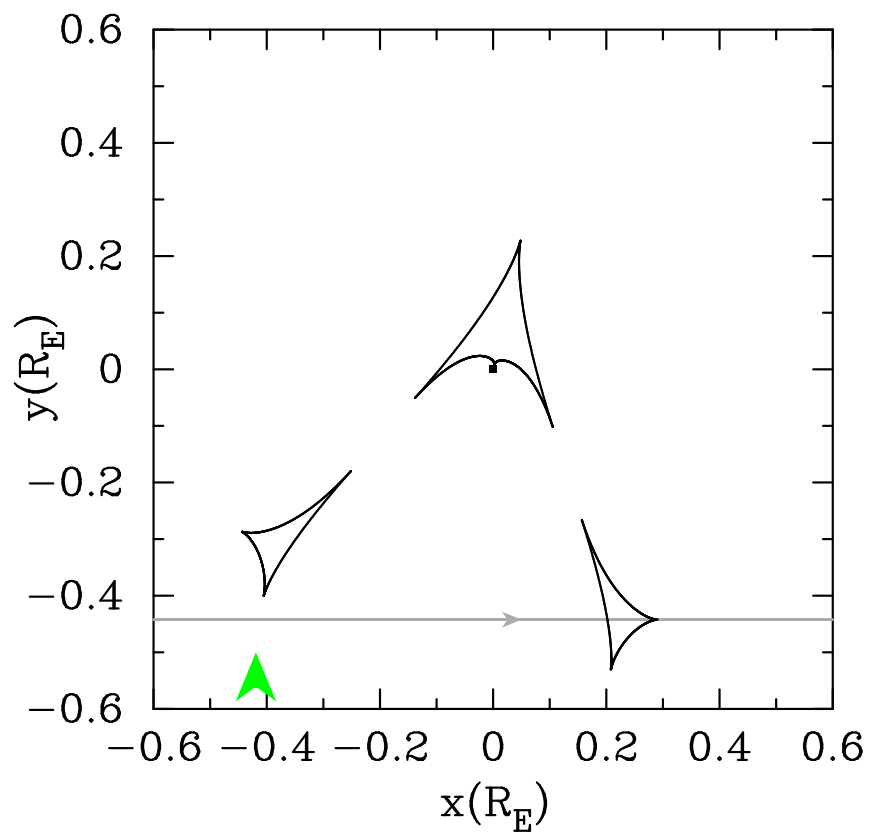

Figure 3.12: Source plane for a binary lens configuration. The primary lens position and the caustic curves are shown as black dots and lines respectively. The source track is given as a grey line, with an arrow indicating the direction of motion. 


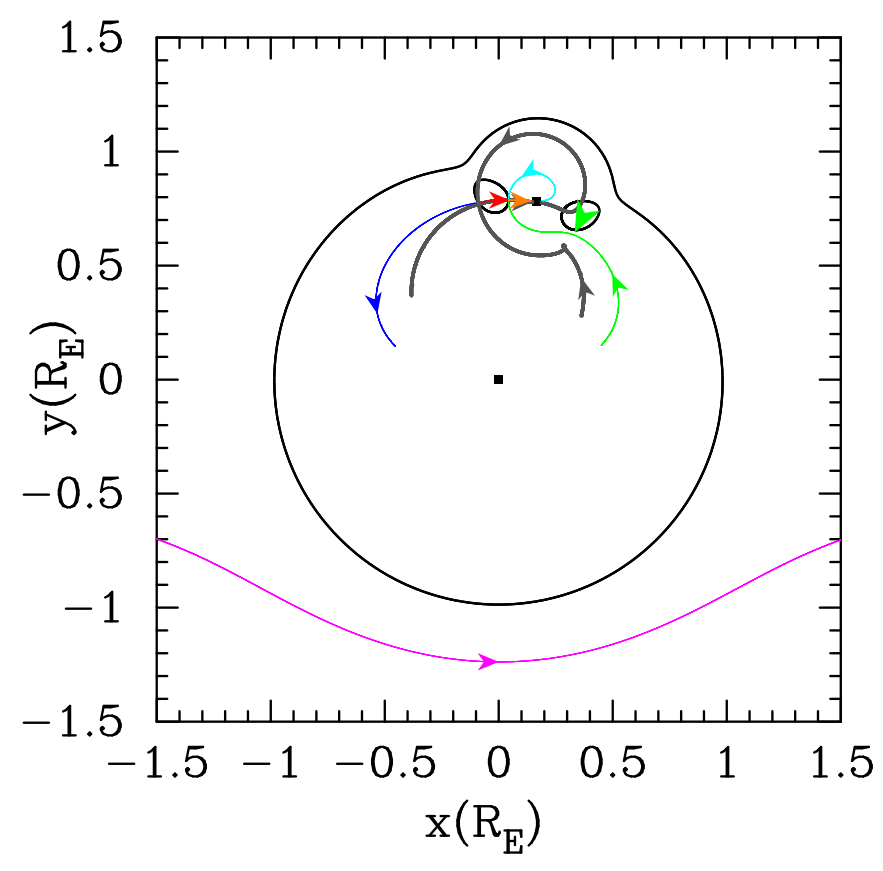

Figure 3.13: Image plane for binary lens configuration. Lens positions and critical curves shown as black dots and lines respectively. Physical images and unphysical roots shown as coloured and grey line respectively. The colour of each physical image correspond to the its magnification in Figure 3.15. The arrows indicate the direction of the images as the source moves from left to right.

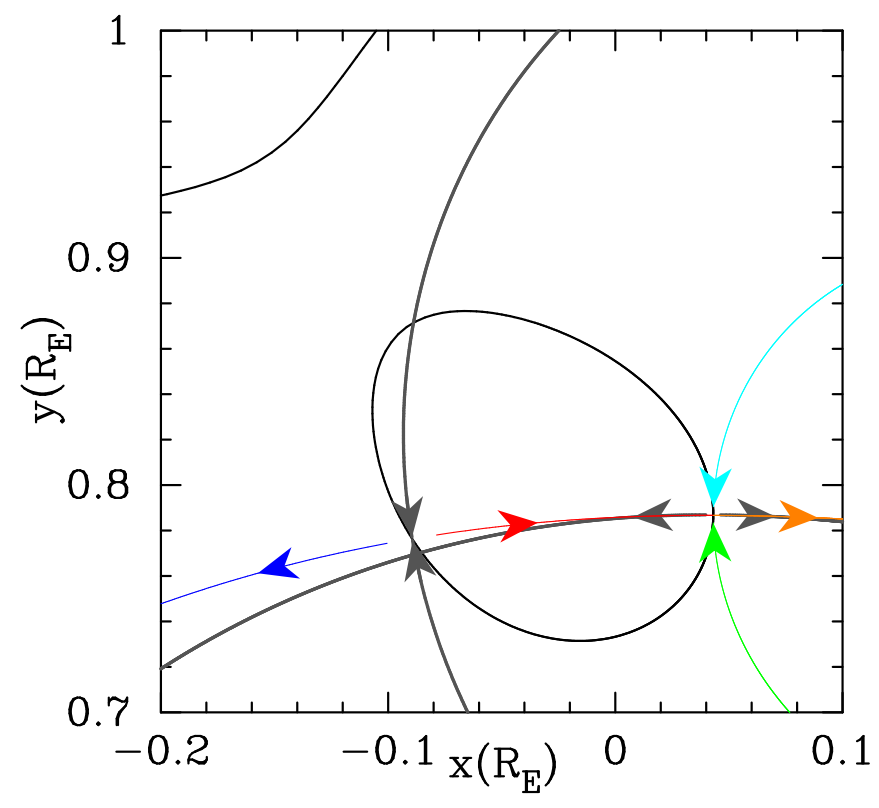

Figure 3.14: Close up of the image plane for the event in Figure 3.13, in the vicinity of a planetary lens. The critical curves are shown by the black lines. Physical images and unphysical roots are shown as coloured and grey lines respectively. The colour of each physical image correspond to the its magnification in Figure 3.15. The arrows indicate the direction of the images as the source moves from left to right. 


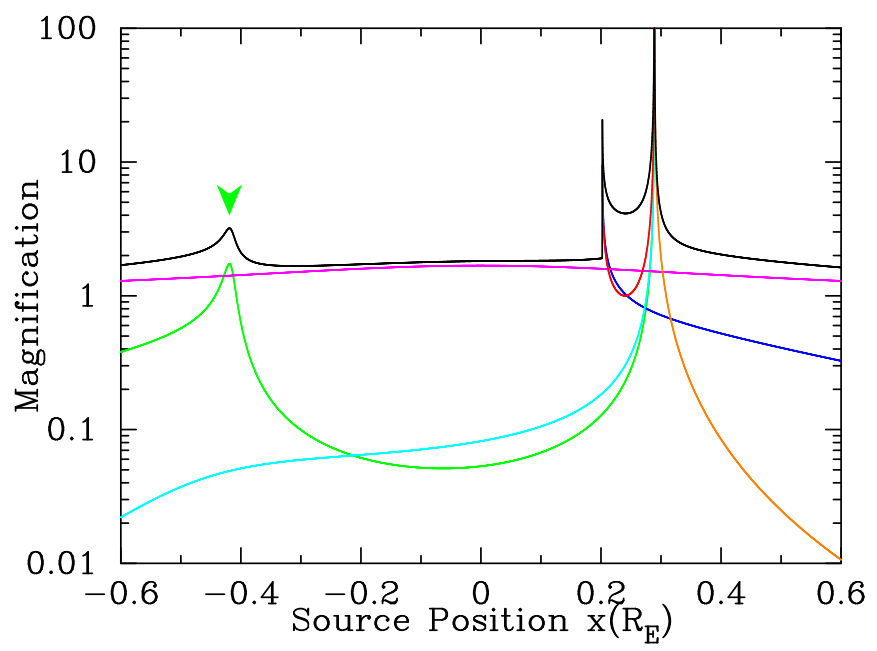

Figure 3.15: Lightcurves of the event in Figures 3.13 and 3.14. Total source magnification is given in black, with magnification of each (physical) image in its respective colour.

Whenever the source crosses a caustic fold, going from $\mathcal{D}^{0}$ to $\mathcal{D}^{1}$, two unphysical roots meet and disappear at a point on the critical curve, and two physical images appear from this position on the critical curve. When the source goes from $\mathcal{D}^{1}$ to $\mathcal{D}^{0}$, the opposite happens, where physical images disappear and become two unphysical roots. . The two of physical images are always travelling in opposite directions at the point of intersection, as are the two of unphysical roots. The directions of the physical roots at this point are always perpendicular to the direction of the unphysical roots when this occurs. This is demonstrated in Figure 3.14, where two grey unphysical roots converge at the critical curve, followed by the red and blue physical images emerging from this point on the critical curve.

The positive-negative image pair that appears when the source enters $\mathcal{D}^{1}$ may or may not have roots in common with the positive-negative pair that disappears when it leaves $\mathcal{D}^{1}$. If the source enters and then exits $\mathcal{D}^{1}$ via the same caustic fold, the same positive-negative pair of images will appear and disappear at the critical curves. However, it is not possible for a source travelling in a straight line to enter and then exit $\mathcal{D}^{1}$ via the same caustic fold since the caustic folds are concave. When the source crosses the planetary caustics in a close binary lens, the same positive image will appear and disappear at the (planetary) critical curves, but the negative image will be different if the source enters and leaves 
via different caustic folds. The positive image may or may not different.

Whenever a source moves from $\mathcal{D}^{0}$ to $\mathcal{D}^{1}$ through a caustic cusp, one physical image and two unphysical roots converge at a critical curve and disappear to be replaced by three physical images. In these situations, the source must move along the axis of the caustic cusp, since the caustic cusps represent a $360^{\circ}$ turn in the caustic curves. The initial physical image therefore moves at the normal to the critical curves and is located outside the critical loop. The two initial unphysical roots also move at the normal to the critical curves, one inside and one outside the critical loop, moving towards each other. After the source has crossed the caustic cusp, two of the new physical images move tangential to the critical curves, and move away from each other, while the other physical image moves at a normal to the critical curves inside the critical curve loop, as though continuing the motion of the initial image. When the point source goes from $\mathcal{D}^{1}$ to $\mathcal{D}^{0}$ through a caustic curve, the reverse happens, where three physical images and an unphysical root disappear at the critical curve to become three unphysical roots and a physical image, travelling in opposite directions to the original roots.

Figures 3.12 to 3.15 also present a point source passing through the low magnification trough, where the total source magnification is lower than the expected corresponding single lens source magnification. These diagrams reveal that the green and cyan images are most responsible for this drop in source magnification, as these images travel between the planetary critical curves, passing close to the planetary lens. As the image moves closer to the lens, the magnification of this image decreases.

These diagrams show the source crossing a planetary caustic with images appearing and disappearing at the critical curves. When the source crosses the central caustic, or the 'central' part of the resonant caustic, these images appear and disappear around the 'central' part of the critical curves, close to the Einstein ring.

By observing the behaviour of the image positions in these cases, we can summarise the behaviour into the following rules:

1. A smooth and continuous source track produces smooth and continuous 
root tracks.

2. The physical images only appear or disappear at the critical curves. Hereafter, a position on the critical curve where these images appear or disappear will be called 'junctions'.

3. The number of roots at each 'junction' is conserved, i.e. the number of physical + unphysical roots entering a 'junction' is equal to the number of physical + unphysical roots leaving the junction.

4. Parity at each 'junction' is conserved.

5. When a pair of physical or unphysical roots is entering a 'junction', the roots are moving directly toward each other. The roots that leave the 'junction' will travel perpendicular to these initial roots.

\subsubsection{Analysing Real Lightcurves}

While data from observed lightcurve events is analysed computationally, we can identify lightcurve features by eye and use our knowledge of these features to give us a rough idea of the caustic curve structure and the lens configuration in these microlensing events. An example of this can be demonstrated using the lightcurve from the OGLE-2005-BLG-390 event shown in Figure 3.16. This lightcurve can be described as a single lens lightcurve with a small bump on the side. The main peak corresponds to the source passing primary lens, while the small bump corresponds to the source passing the planetary caustics. The main peak occurs around 2453583JD with a height of approximately 3.0. By assuming the mass of the planet is very small and using the approximation $\epsilon \approx 1$, we can us Equation 3.4 to show that the impact parameter is approximately given as $0.348 R_{E}$.

A source magnification of approximately 1.34 corresponds to the time where the source lies on the Einstein ring of the primary lens, which occurs at about 10days from the peak time. The small bump on the lightcurve occurs roughly at this time, therefore the planetary caustics are positioned roughly $1 R_{E}$ from the primary lens. Using Equation 3.21, the lens separation can be estimated 
as $1.618 R_{E}$. Since the impact parameter is not small, the crossing time is a little trickier to estimate. However, Pythagoras' Theorem can show that distance between the impact parameter and the Einstein radius is $0.937 R_{E}$, giving a crossing time of approximately 10.66days. These values are reasonable close to the values $(0.359 \pm 0.005) R_{E},(2453582.731 \pm 0.005) \mathrm{JD},(11.03 \pm 0.11)$ days and $(1.610 \pm 0.008) R_{E}$ for the impact parameter, peak time, crossing time, and lens separation respectively (Beaulieu et al., 2006)

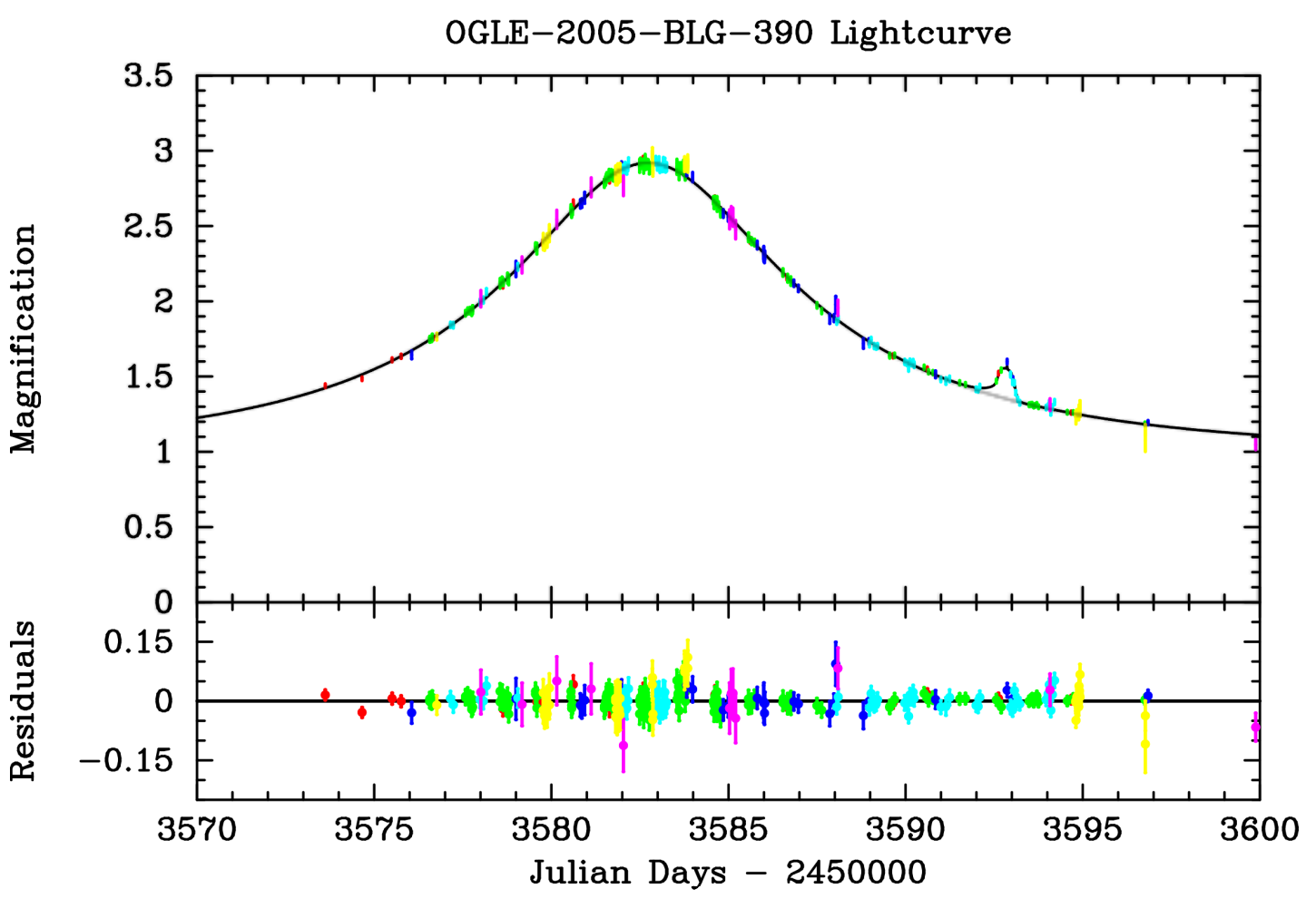

Figure 3.16: Lightcurve for the event OGLE-2005-BLG-390 (orange) with observational data. The lower panel shows the residuals between the observational data and the model lightcurve.

While not all lightcurves can be analysed as easily as this, knowledge of how the source magnification changes with respect to the caustic structure can still reveal important information on the binary configuration. For example, Figure 3.17 shows the best fitting model for the MOA-2009-BLG-387 event (which included parallax and orbital motion) as given by Batista [2011]. This lightcurve generally has the shape of a single lens lightcurve (grey) with a two U-shaped features with a lower source magnification between them. This suggests the source track crosses the caustic curves at the back of the binary lens and through the low magnification trough, implying a planetary binary configuration with a 
lens separation less than $1 R_{E}$. This model had a mass ratio $q=0.0132 \pm 0.0002 R_{E}$ and a lens separation $d=0.9136 \pm 0.0003 R_{E}$.

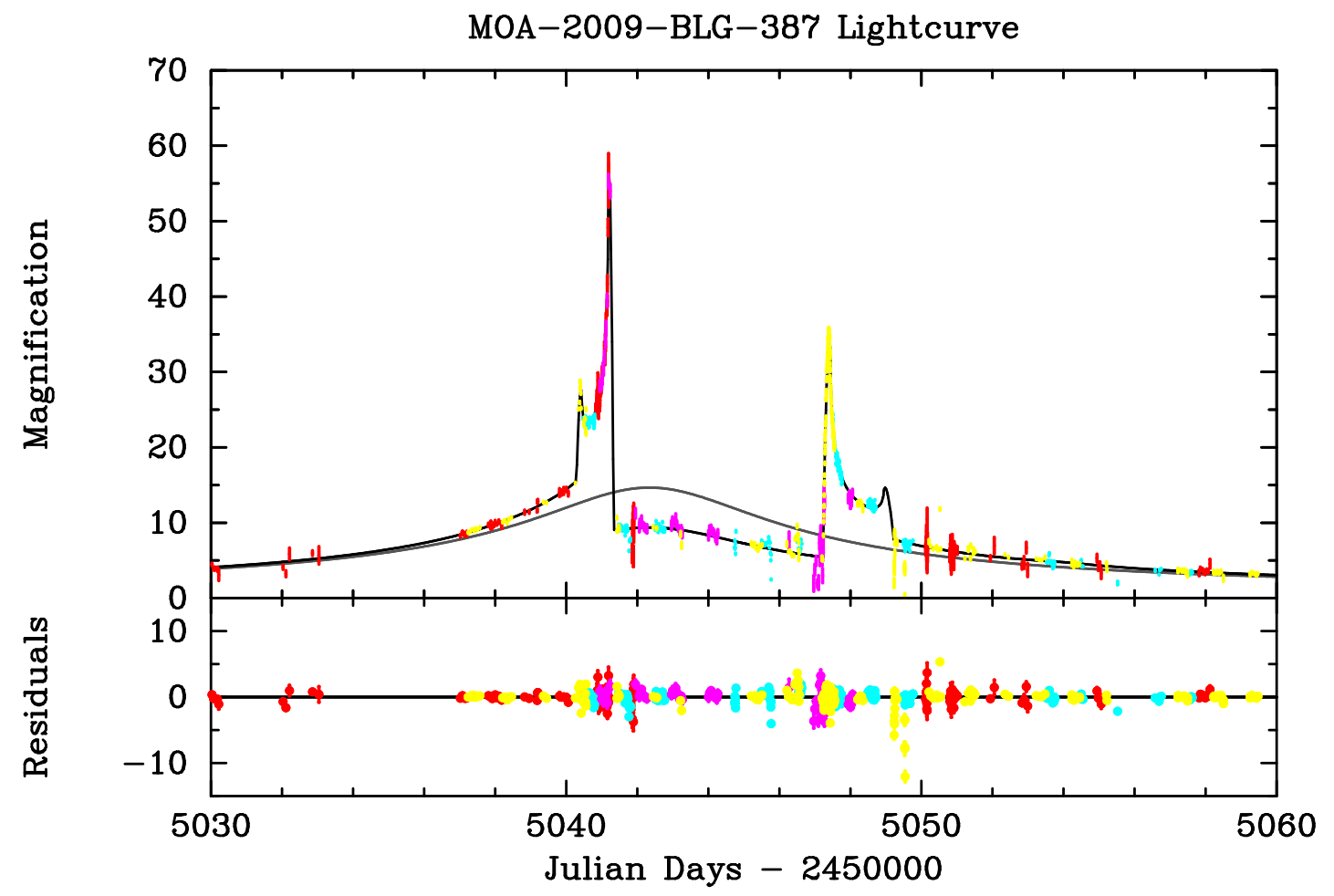

Figure 3.17: Lightcurve for the event MOA-2009-BLG-387 (orange) with observational data. The lower panel shows the residuals between the observational data and the model lightcurve.

\subsection{Higher Lens Configurations}

As seen previously in Figure 3.6, the caustic curves in triple lens and higher configurations can cross over themselves in such a way to create multiple domain caustic structures. This differs from the binary lens case where there is only one caustic domain inside the caustic curves $\left(D^{1}\right)$. Triple and quadruple lens systems have been shown to produce caustic domains up to $D^{3}$ and $D^{5}$ respectively. However, it has been argued by Rhie [2001] that a multiple lens system with $N$ lenses cannot create caustic domains higher than $D^{2 N-3}$.

Figure 3.18 presents the source plane for a system with three lenses with equal masses, showing the source track in grey and the caustic curves in black. Figures 3.19 and 3.20 present the image plane, showing the critical curves in black, and each unphysical root in grey, and each physical image in colour. Figure 3.20 
shows a close up of the image positions near the centre of mass. Figure 3.21 shows the lightcurve for this event and the magnification of each image in its respective colour.

Note that while in $\mathcal{D}^{1}$ the source passes a cusp and enters the caustic domain $\mathcal{D}^{2}$. This is represented on the lightcurve as a peak and a higher $\left(\mathcal{D}^{2}\right)$ U-shaped feature inside the $\mathcal{D}^{1} \mathrm{U}$-shaped feature. This lightcurve with U-shaped features inside other U-shaped features in the lightcurve only occurs in lens configurations with three of more lenses. Identifying the presence of this occurrence in a real observed light curve is an indication that the lightcurve is produced by a lens system with three of more bodies.

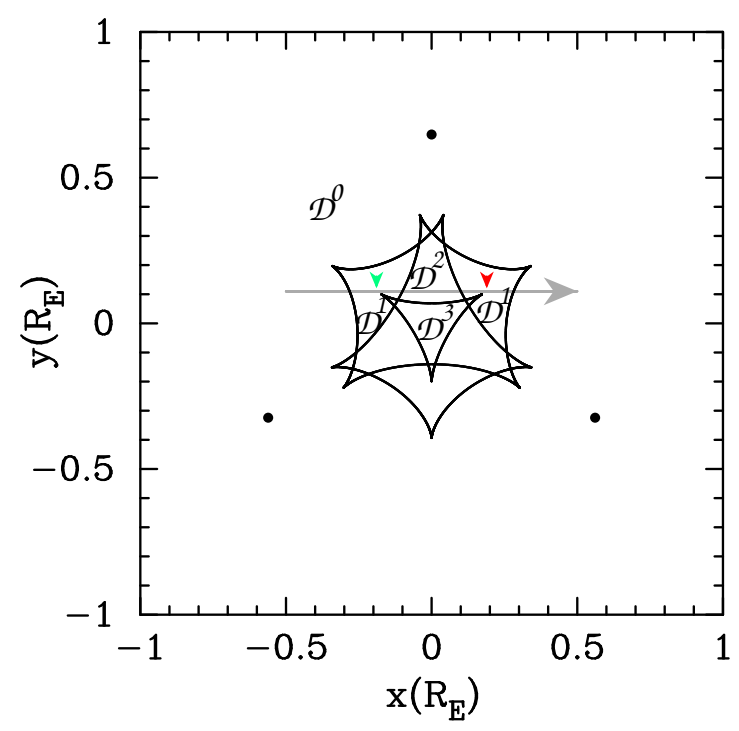

Figure 3.18: Source plane for a triple lens configuration. The lens positions and the caustic curves are shown as black dots and lines respectively. The source track is shown by the grey line and the direction of the source is represented by the arrow.

\subsubsection{Unphysical Root Pairs}

When each root is inverse-ray traced back to the source plane, the roots that correspond to the physical images are roots that inverse-ray trace to the position of the source, whereas roots that are unphysical do not. However, it is interesting to note that the unphysical roots form pairs; two unphysical roots inverse-ray trace to two points on the source plane at equal and opposite displacements from the source. This is shown in Figure 3.22b.

When the source is far from the lenses, the roots are positioned close to 


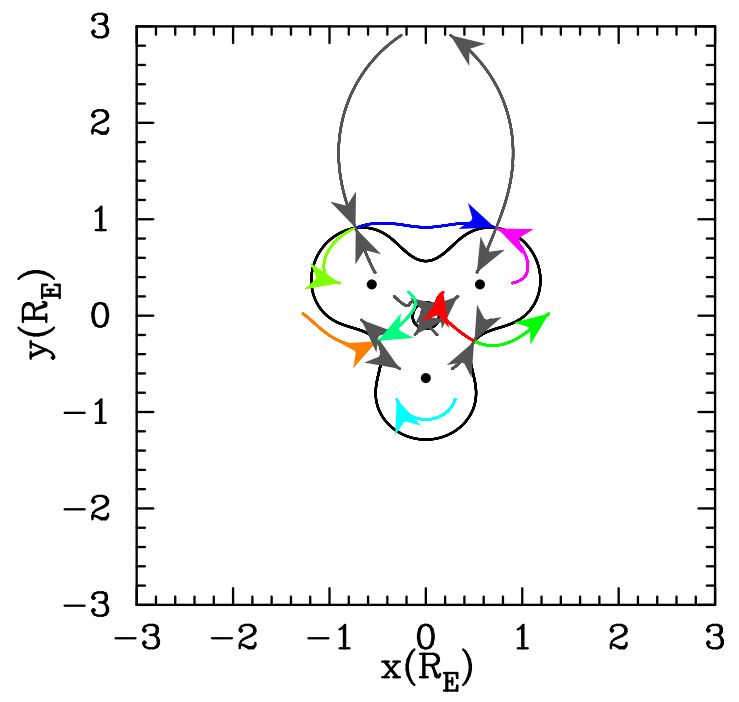

Figure 3.19: Image plane for a triple lens configuration. Lens positions and critical curves shown as black dots and lines respectively. The physical images and unphysical roots are shown as coloured and grey lines respectively. The colour of each physical image correspond to the its magnification in Figure 3.21. The arrows indicate the direction of the images as the source moves from left to right.

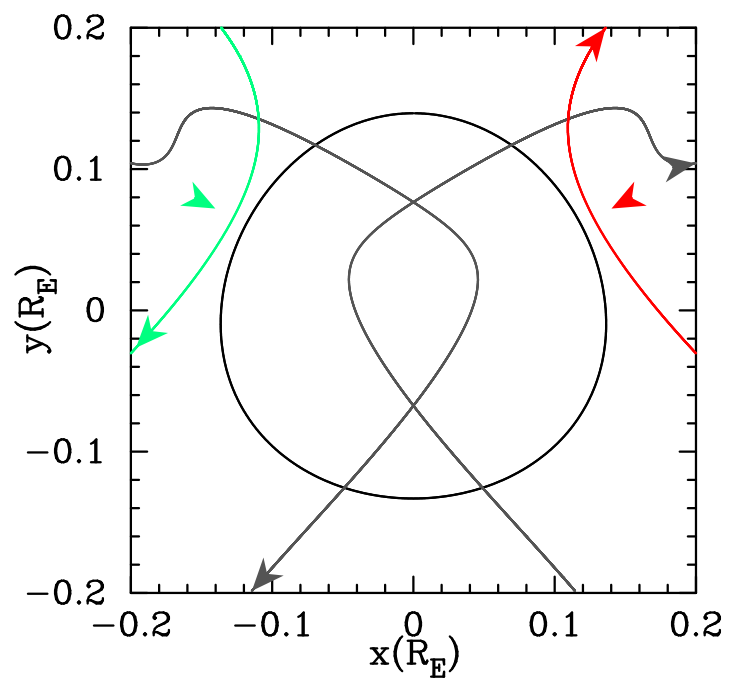

Figure 3.20: Close up of the image plane for a triple lens configuration in vicinity of a planetary lens. The critical curves are shown by the black lines. The physical images and unphysical roots are shown as coloured and grey lines respectively. The colour of each physical image correspond to the its magnification in Figure 3.21. The arrows indicate the direction of the images as the source moves from left to right. 


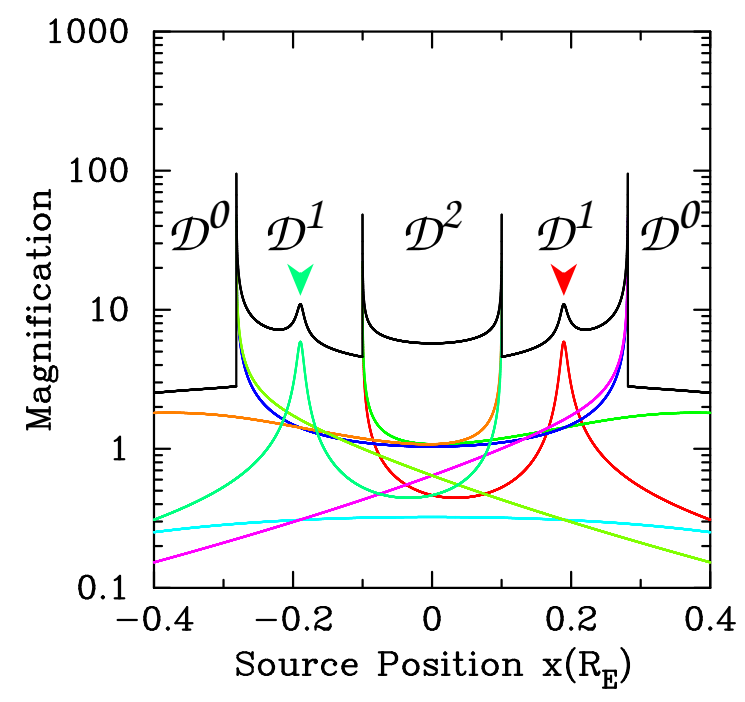

Figure 3.21: Lightcurve of the event in Figures $3.18-3.20$. The total source magnification is shown in black, with magnification of each (physical) image given in its respective colour.

the lenses. In this situation, each root from an unphysical pair is close to a different lens from its partner, and every unphysical pair has a different lens-pair combination. This is shown in Figure 3.22a. Therefore, there are a maximum number of $N(N-1) / 2$ pairs of unphysical roots, giving a maximum of $N^{2}-N$ unphysical roots, and there are $N-1$ unphysical roots close to each lens, each from a different pair. This agrees with the result shown in section 2.5.
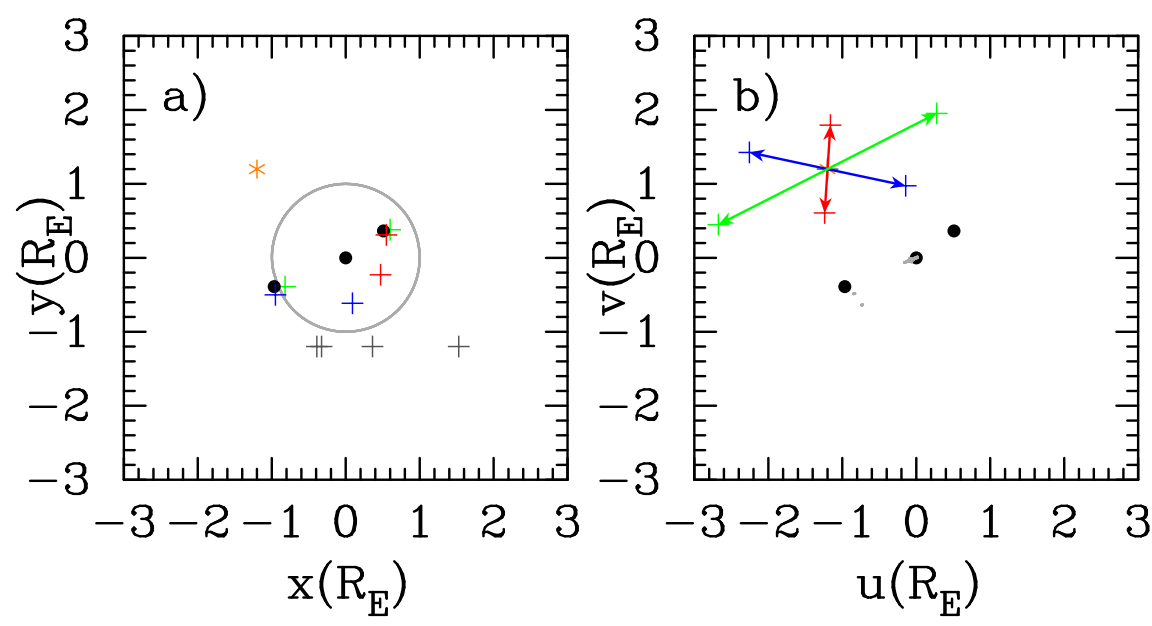

Figure 3.22: The root positions and unphysical pairs for a 3 lens system. The critical and caustic curves are shown in light grey, and the position of the source is shown by the orange asterisk. The lenses and physical images are represented by the black dots and dark grey crosses respectively. One pair of unphysical roots is represented by the two blue crosses, another pair by the two red crosses, and another pair by the two green crosses. (a) shows the root positions on the image plane, and (b) shows the image positions inverse-ray traced to the source plane. 


\subsubsection{Multiple Planetary Events}

While systems with three or more stars with equal masses are very rare and unlikely to be discovered in the near future, a triple lens system consisting of a star and two planets has been confirmed (paper ob06109), and another has recently been observed (OGLE-2012-BLG-0026). Therefore, the lens equation and the equations derived from it are far more useful when they use planetary mass ratios.

Planetary mass ratios are typically on the order of $10^{-3}-10^{-5}$. Therefore, we can use the approximation $\epsilon_{1}>>\epsilon_{j}$ with $j \geq 2$ and $\epsilon \approx 1$ to decompose the multiple planetary lens equation into first and second order approximations, corresponding to single lens and binary lens configurations respectively. The first order approximation is given by,

$$
w \approx z-\frac{1}{\bar{z}-\overline{r_{1}}}
$$

Given a planetary system with $N-1$ planets, there are $N-1$ second order binary lens approximations. Each binary lens approximation can be defined by,

$$
w \approx z-\frac{\epsilon_{1}^{(j)}}{\bar{z}-\overline{r_{1}}}-\frac{\epsilon_{j}^{(j)}}{\bar{z}-\overline{r_{j}}}
$$

where $\epsilon_{j}^{(j)}$ is given by,

$$
\epsilon_{j}^{(j)}=\frac{\epsilon_{j}}{\epsilon_{1}} \quad j \geq 2
$$

so that the mass ratios still add to unity.

The source position $w$ calculated using this binary lens approximation will have an error at an order of magnitude no less than the planetary mass fractions $\left(\epsilon_{j}\right)$. The redefine mass fractions $\epsilon_{j}^{(j)}$ and the initial mass fractions $\epsilon_{j}$ will differ on the order of the planetary mass fractions $\left(\epsilon_{j}^{2}\right)$. Therefore, redefining the mass fractions will have no significant effect on the approximation. Redefining the mass fractions using Equation 3.25 is a matter of convenience, as the parameters for a microlensing event usually give the mass ratio, not the mass fraction, and 
the expressions for the coefficients of the lens and critical curves polynomials assume the mass fractions add to unity.

\subsubsection{Additive Nature of Caustic and Critical Curves}

The critical and caustic curves for a system with $N$ lenses can be approximated using the critical and caustic curves of several binary lens. The positions of these curves can be derived from the binary lens approximation given in Equation 3.24. The first order approximation for the critical curves and caustic curves are the Einstein ring and a point at the primary lens respectively.

As mentioned earlier in the chapter, the critical curve polynomial for an $N$ lens system has $2 N$ roots for each value of $\phi$. Figures 3.23 and 3.24 respectively show the critical curves and caustic curves separated into their corresponding roots. The black curves in all six boxes correspond to the six critical curve roots of the triple lens, consisting of the primary lens at the origin (represented by the grey dot) with both planets (represented by the red and cyan dots, with mass ratios $\epsilon_{2} / \epsilon_{1}=0.001$ and $\epsilon_{3} / \epsilon_{1}=0.0005$ respectively). The red and cyan curves correspond to the two binary lenses. The curves for the binary lens configuration consisting of the primary lens with the red planet are presented by the red curves while the curves binary lens configuration consisting of the primary lens and the cyan planet are presented by the cyan colours. The grey curves describe the critical curves (or Einstein ring) for the single lens at the position of the primary lens (the origin). These figures show that the separate roots correspond to the "central" and "planetary" parts of the curves, as described above: boxes a) and b) show the black, red, cyan curves corresponding to primary lens. The curves generally follow the grey Einstein ring, except when these line deviate close to the planetary lens. Boxes d) and e) show the black and red curves corresponding to the red planet, and boxes c) and f) show the black and cyan curves corresponding to the cyan planet.

Figure 3.25 shows a close up of the triple and binary lens caustic curves. The caustic curves from these binary lens configurations are offset by approximately $0.001 R_{E}$ and $0.0005 R_{E}$. This demonstrates that, for multiple planetary lenses 
(i.e. small mass ratios), the triple lens caustic curves are better approximated by the binary lens caustic curves corresponding to the largest planetary mass, than by the binary lens caustic curves corresponding to the smaller planetary masses. The offset of the caustic curves of a particular binary lens approximation from these of the multiple lens approximation is generally on the order of the mass ratio of the planet. However, the main contributor of this offset could be the changes in the centre of mass between the binary and triple lens systems.
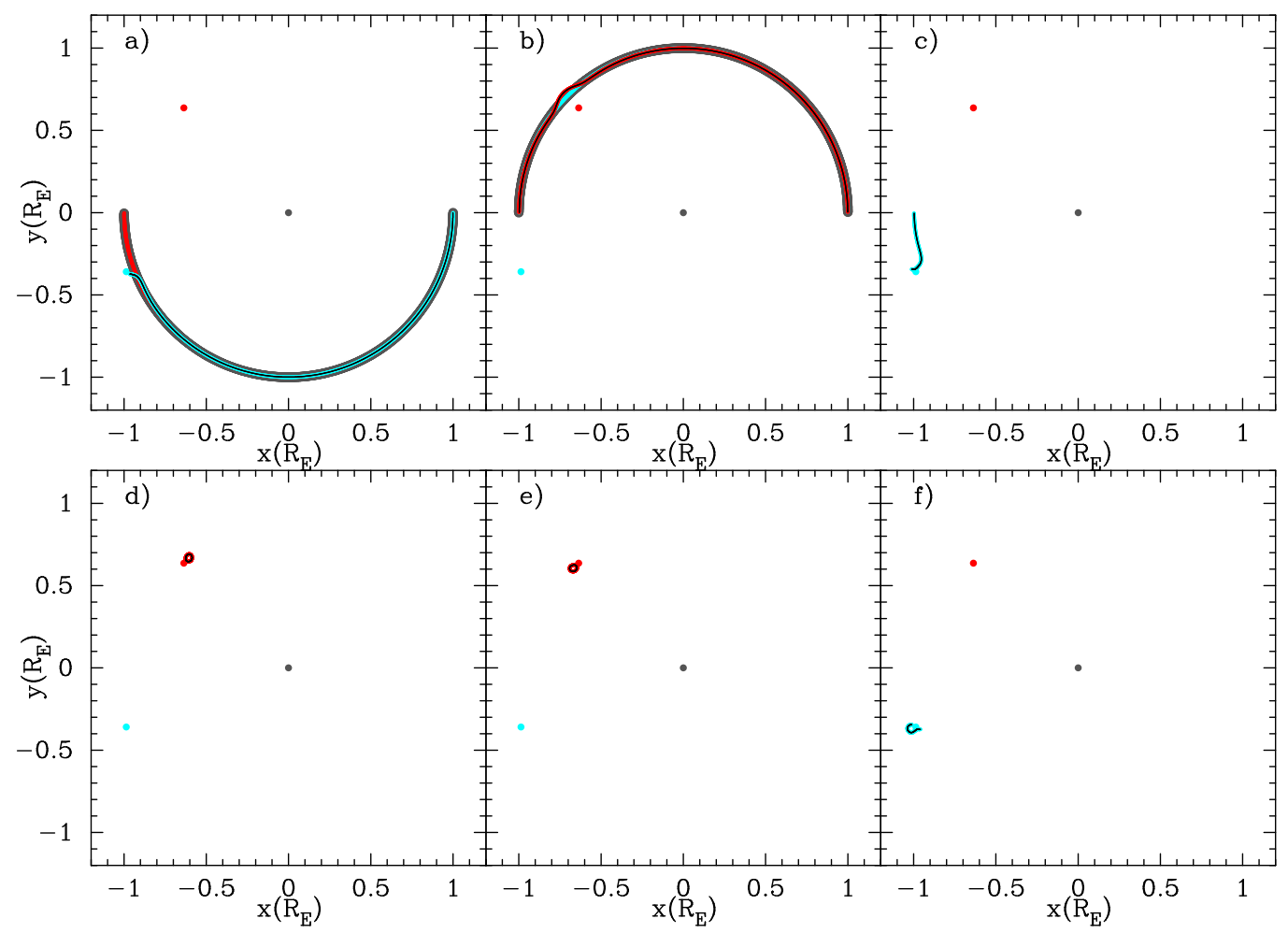

Figure 3.23: Critical curves and decomposition curves for a triple lens. The critical curves for the complete configuration are represented in black. The critical curves for each binary lens consisting of the primary lens with the planetary lens are represented in the same colour as their respective lenses: red or cyan. The grey curves represent the Einstein ring for a single lens located at the origin.

\subsubsection{Additive Nature of Planetary Lightcurves}

Just as the caustic and critical curves of multiple planetary configurations can be approximated using the caustic and critical curves from multiple individual binary lens configurations, the lightcurves produced by multiple planetary events can also be approximated using multiple individual binary lens lightcurves (Rattenbury et al., 2002; Han, 2005). Suppose we define $A_{1}(t)$ as the lightcurve of 

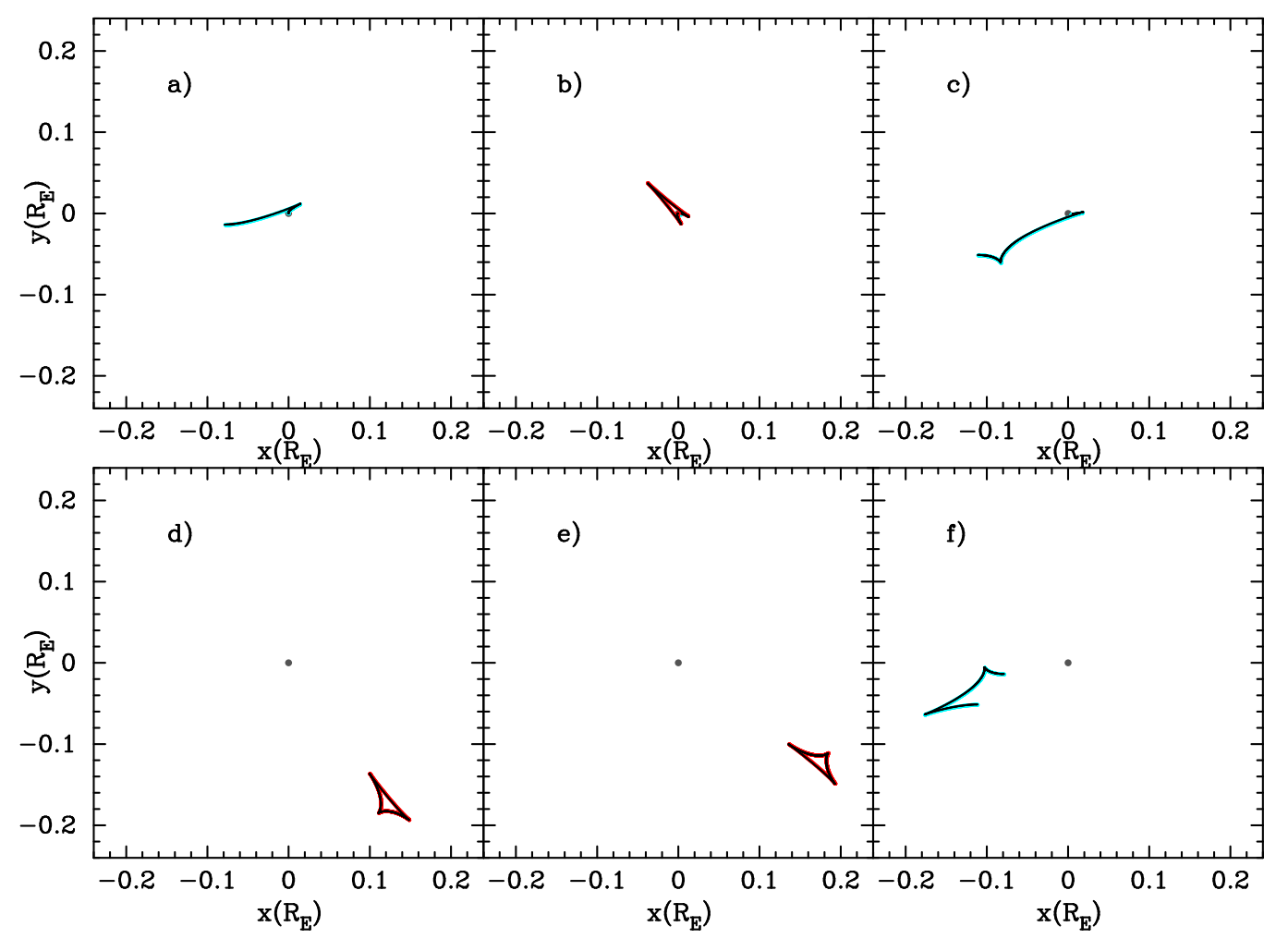

Figure 3.24: Caustic curves and decomposition curves for a triple lens. The caustic curves for the complete configuration in black. The caustic curves for each binary lens consisting of the primary lens with the planetary lens are represented in the same colour as their respective lenses: red or cyan.
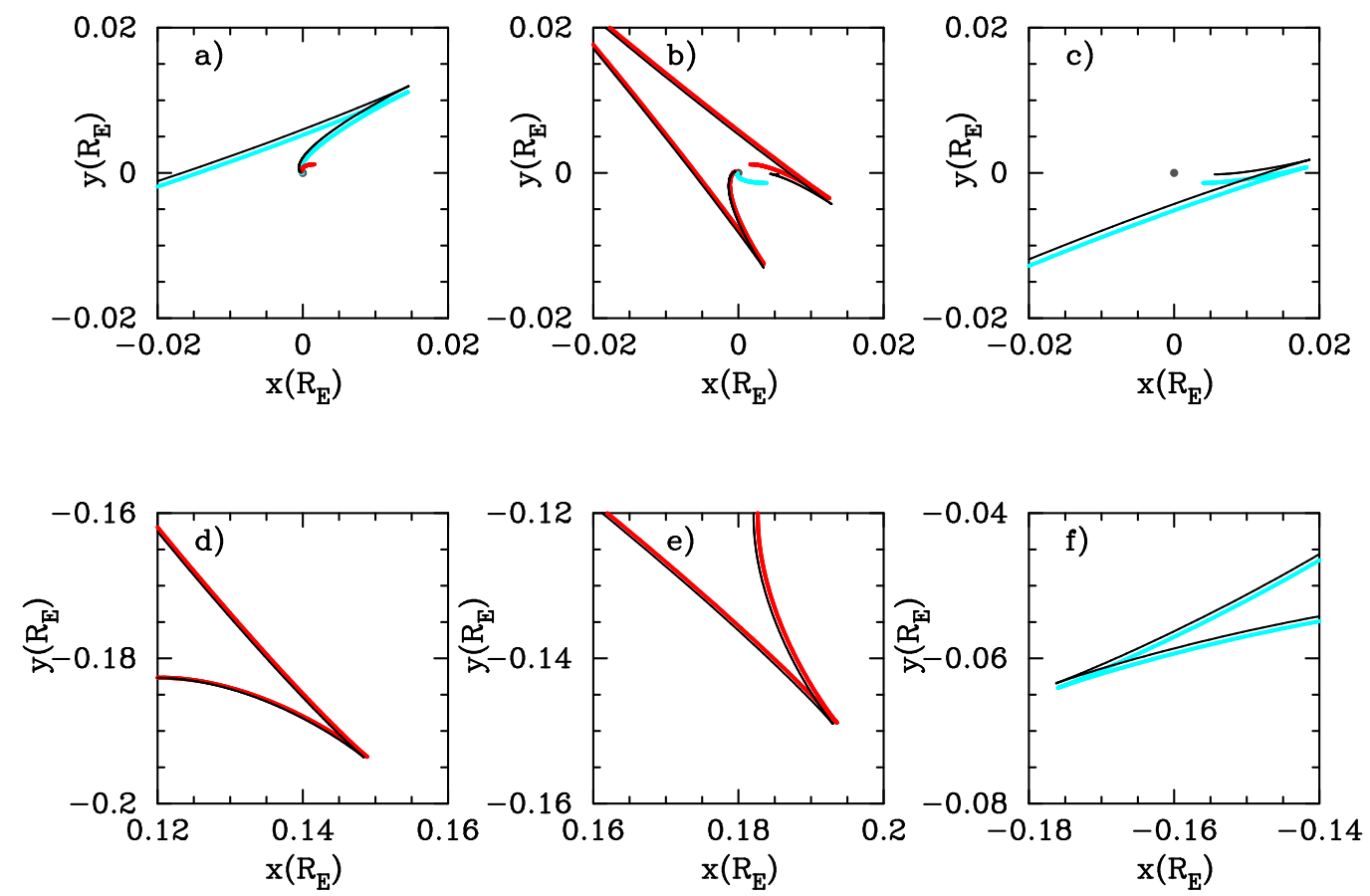

Figure 3.25: Close up of Figure 3.24 showing the caustic curves for the complete configuration in black. The critical curves for each binary lens consisting of the primary lens with the planetary lens are represented in the same colour as their respective lenses: red or cyan. 
the corresponding single lens, and $A_{1 j}(t)$ as the lightcurves corresponding to the binary lenses consisting of the primary lens and the $j$ th lens (planetary). The final source magnification $A(t)$ of the $N$ lens event can then be approximated as,

$$
A(t) \approx\left[\sum_{j=2}^{N} A_{1 j}\right]-(N-1) A_{1}(t) .
$$

This is equation is demonstrated in Figures 3.26 to 3.28. Figure 3.26 presents a triple planet configuration (quadruple lens) with the primary lens in violet and the planetary lenses in red, cyan and chartreuse, with mass ratios $q=0.001$, 0.0005 , and 0.0002 respectively. In this diagram, the critical curves are shown by the dark grey curves, the caustic curves by the black curves.

Figure 3.27 presents a close up of the (black) caustic curves of the quadruple lens along with the caustic curves for the three binary lenses each consisting of the primary lens and one planetary lens. The red, cyan and chartreuse caustic curves correspond to binary lens configurations that consist of the primary lens and the red, cyan and chartreuse green planetary lenses respectively, and the source track by the light grey line. Figure 3.28 then shows the lightcurves produced by these configurations in their respective colours. The violet lightcurve represents the lightcurve calculated using Equation 3.26, showing that the overall lightcurve can be reasonably approximated by this expression.

These diagrams demonstrate that the Equation 3.26 can give the approximate shape of the lightcurve, however, just as the caustic curves of the binary lens are offset from the caustic curves of the multiple planetary configuration, the features in the binary lens light curves are offset from the multiple planetary configuration, and this offset is on the order of the mass ratios. When analysing a real microlensing event, the lightcurve must be calculated with a resolution much smaller than the order of the mass ratio of the planet. Therefore, the approximation given in Equation 3.26 should not be used directly when modelling a real microlensing event. However, this type of deconstruction into binary lens configurations can be used to help identify the parts of the caustic structure associated with each planet (Gaudi et al., 2008). 


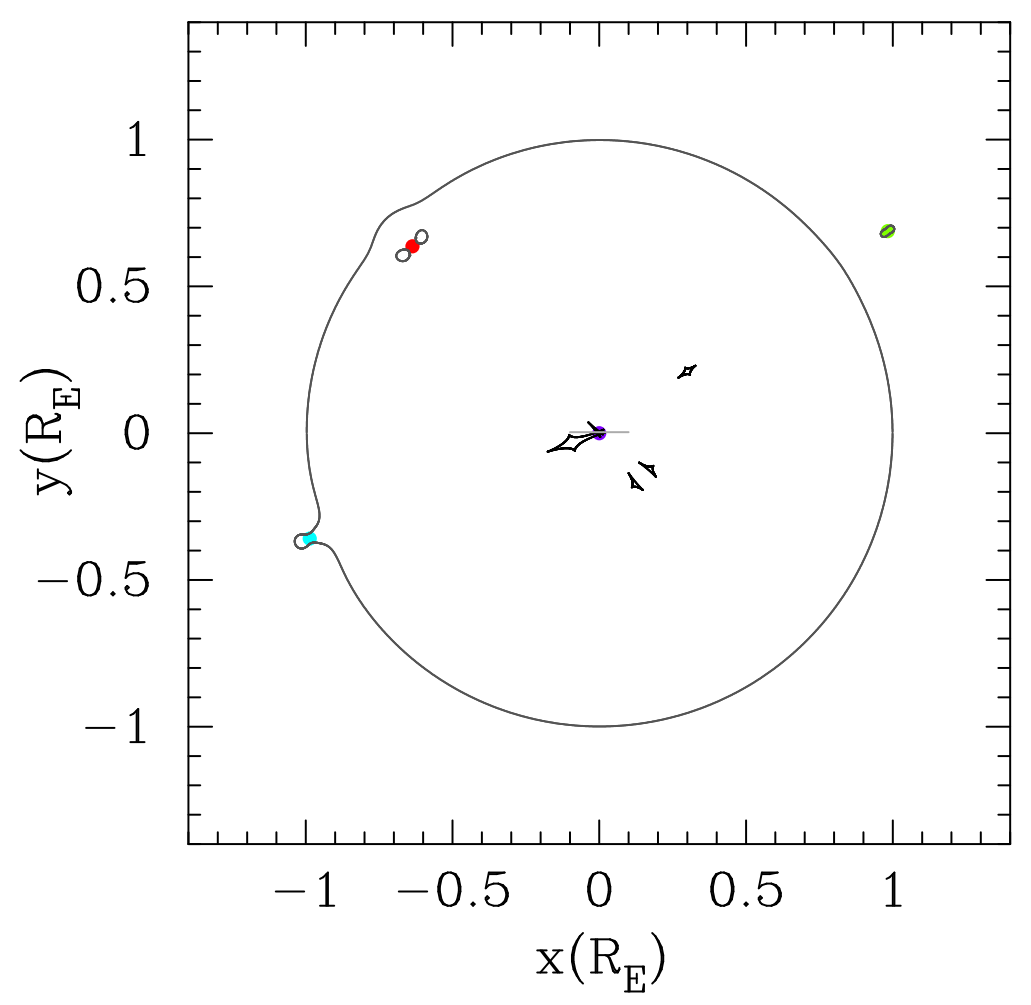

Figure 3.26: A quadruple lens configuration showing the critical curves (grey), the caustic curves (black). The primary lens is represented by the dark grey dots at the origin, and the planetary lens positions are represented by the red, cyan and chartreuse green dots respectively.

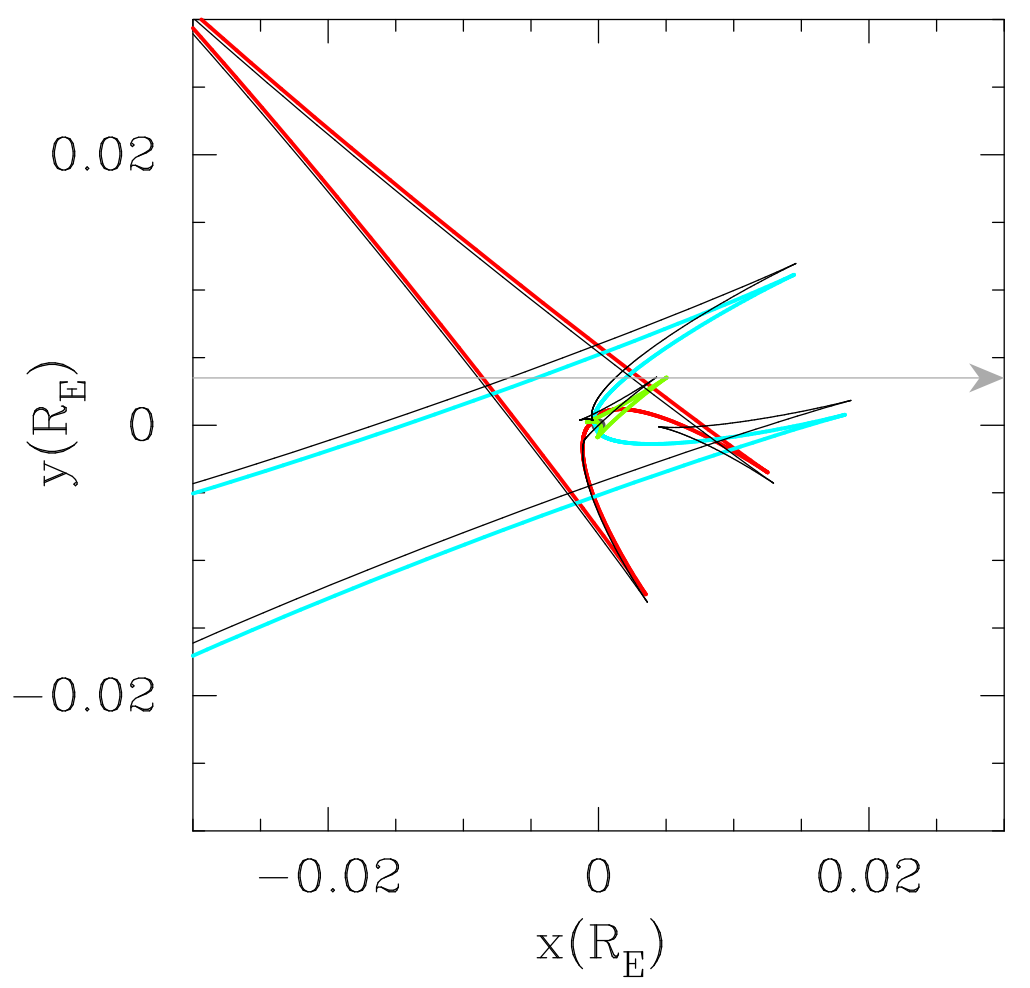

Figure 3.27: Close up of Figure 3.26 showing the caustic curves for the complete configuration in black. The critical curves for each binary lens consisting of the primary lens with the planetary lens are represented in the same colour as their respective lenses: red, cyan or chartreuse green. 


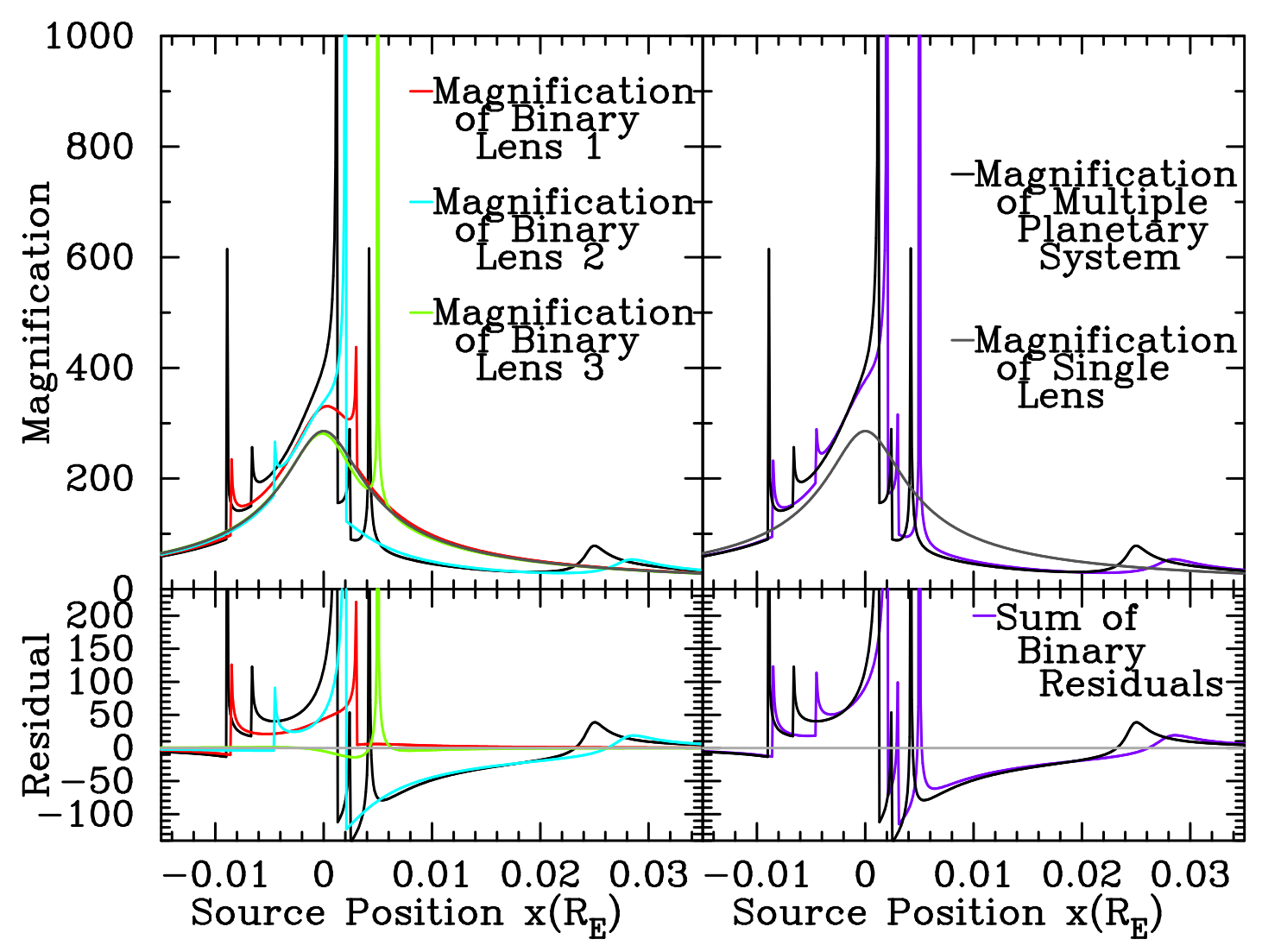

Figure 3.28: The panels on the left show the lightcurves for a quadruple planet configuration, along with each binary lens lightcurve and the residuals from the single lens lightcurve. The panels on the right show the sum of the residuals and the corresponding lightcurve compared with the triple planet lightcurve. 


\section{Chapter 4}

\section{Finite Source Effects}

In Chapter 4 we showed how the magnification can be calculated by taking the limit where the images and source are shrunk to points on the complex plane. While this approximation is simple, it sometimes fails to give an appropriate estimate for the magnification. Examples of this include when the point source crosses a caustic curve (or caustic point in the single lens case) where the point source magnification diverges significantly. In these cases, a source with a finite size must be used to give an accurate calculation of the magnification.

In this chapter we investigate methods to approximate the magnification for a finite size source. We start by introducing the concept of a source disk and showing the effects a finite source has on a theoretical lightcurve. We then investigate the concept of limb-darkening, where the brightness of the source disk varies across its surface, darkening at the edges (or 'limbs') of the star. We shall see later that this effect is significant for large source disk, especially as the source crosses a caustic curve. Next, we show how the source disk can be approximated as a polygon, and show how this can be used most effectively to calculate the image areas and magnifications. We then introduce another family of methods, the multipole approximations (in particular the quadrapole and hexadecapole approximations), which approximate a finite source magnification using a small number of point source magnifications. This chapter concludes by discussing which method was found to be the optimal method for calculating the magnification when modelling microlensing events. 


\subsection{Finite Source Disk}

Point source approximations work well in most cases when the source is at a reasonable distance from the caustic curves, but as the source approaches the caustic curves the point source magnification diverges. In these cases, the finite size of the source star becomes important. To approximate this finite source, the source star can be modelled as a disk with radius $\rho$. The source disk represents the physically spherical star projected onto a 2-dimensional plane. The images are therefore represented by the distorted projections of this source disk on the image plane, so the magnification is calculated simply as the ratio of the areas of these distorted images to the area of the source disk $\pi \rho^{2}$. However, the area of these images cannot be calculated analytically, so numerical approximations must be used. These two numerical approximations, the polygon and multipole approximations will be discussed later in Sections 5.2 and 5.3.

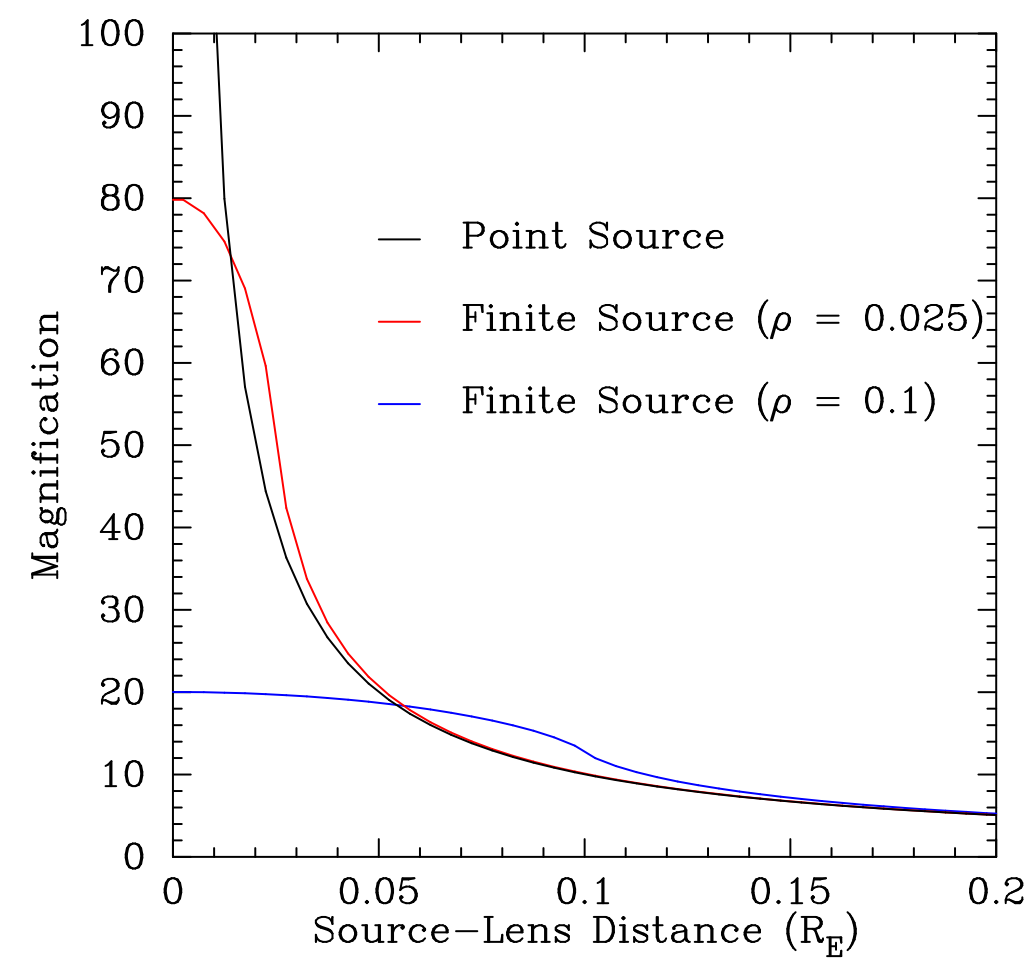

Figure 4.1: Magnification over a range of source-lens separations by a single lens, where the black, red and blue lines represent the magnifications for a point source, and finite source disk with radius $0.025 R_{E}$, and a finite source disk with radius $0.1 R_{E}$.

Figure 4.1 shows the magnification plotted against the source-lens separation for a single lens. In this plot, the magnification is calculated using three different 
sized sources: a point source, a finite source with radius $\rho=0.025 R_{E}$, and a finite source with radius $\rho=0.1 R_{E}$. The two finite sources were calculated using the polygon method discussed later in this chapter. These plots show that a larger finite source disk produces a single lens lightcurve that is generally shorter and wider than the lightcurve from a small source disk. When the finite source is small, the lightcurve shows little difference from the point source lightcurve except at very small source-lens separations. When the source-lens separation zero (i.e. the source and lens are in the same position on the source/image pane), the magnification $A$ of the finite disk can be given as,

$$
A=2 \sqrt{\frac{1}{\rho^{2}}+\frac{1}{4}} .
$$

A large finite source with a multiple lens has a similar effect on the lightcurve, making the peaks shorter and wider. However, if the source disk becomes too large, the features in the lightcurve are washed-out and individual cusp and caustic crossings may merge together, or become lost altogether, as demonstrated in the figures below.

Figure 4.2 shows the critical curves and caustic curves for a triple lens system (a host star with two planets) and Figure 4.3 shows a close up of the caustic curves with the source track. The arrows in Figure 4.3 indicate where the source crosses a caustic curve or passes a caustic cusp. These arrows correspond to the arrows in Figure 4.4, which indicate peaks in the lightcurve for this event. The black lightcurve represents the point source magnification, and the red and blue lines represent the lightcurves for two finite source disks with radii $0.005 R_{E}$ and $0.02 R_{E}$ respectively. These sizes for source disk radii are within the range of typical source disk radii in real microlensing events. The lightcurve with a source radius of $0.005 R_{E}$ has peaks that are much shorter (and a little wider) than the lightcurve with the point source. The lightcurve with a source radius of $0.02 R_{E}$ demonstrates that the peaks become washed-out and start merging together when the source becomes too large. A lightcurve associated with a large source disk such as this is often hard to analyse, as there is a lack of clarity in the peak positions and hence a large uncertainty in the positions of the caustic curves. 


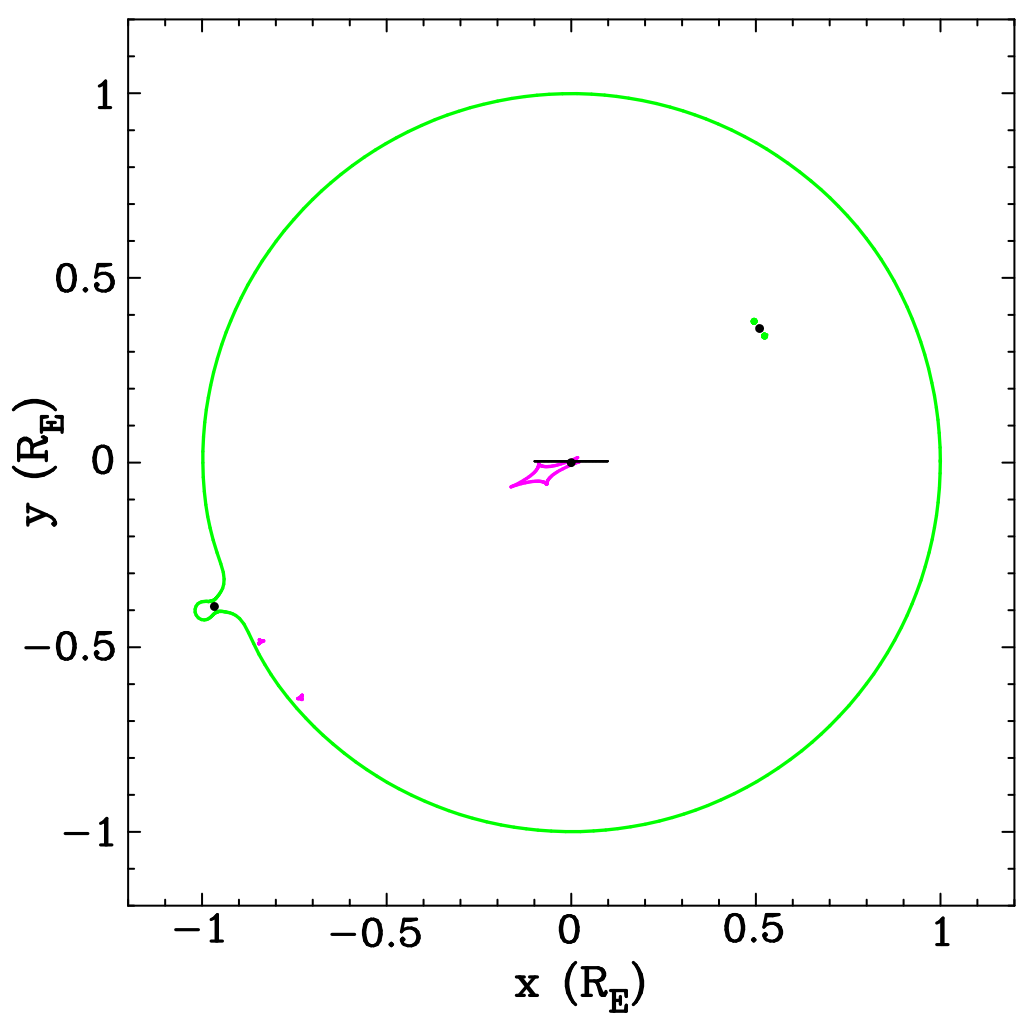

Figure 4.2: A triple lens event showing the lenses in black, the critical curves in green, the caustic curves in magenta, and the source track in black.

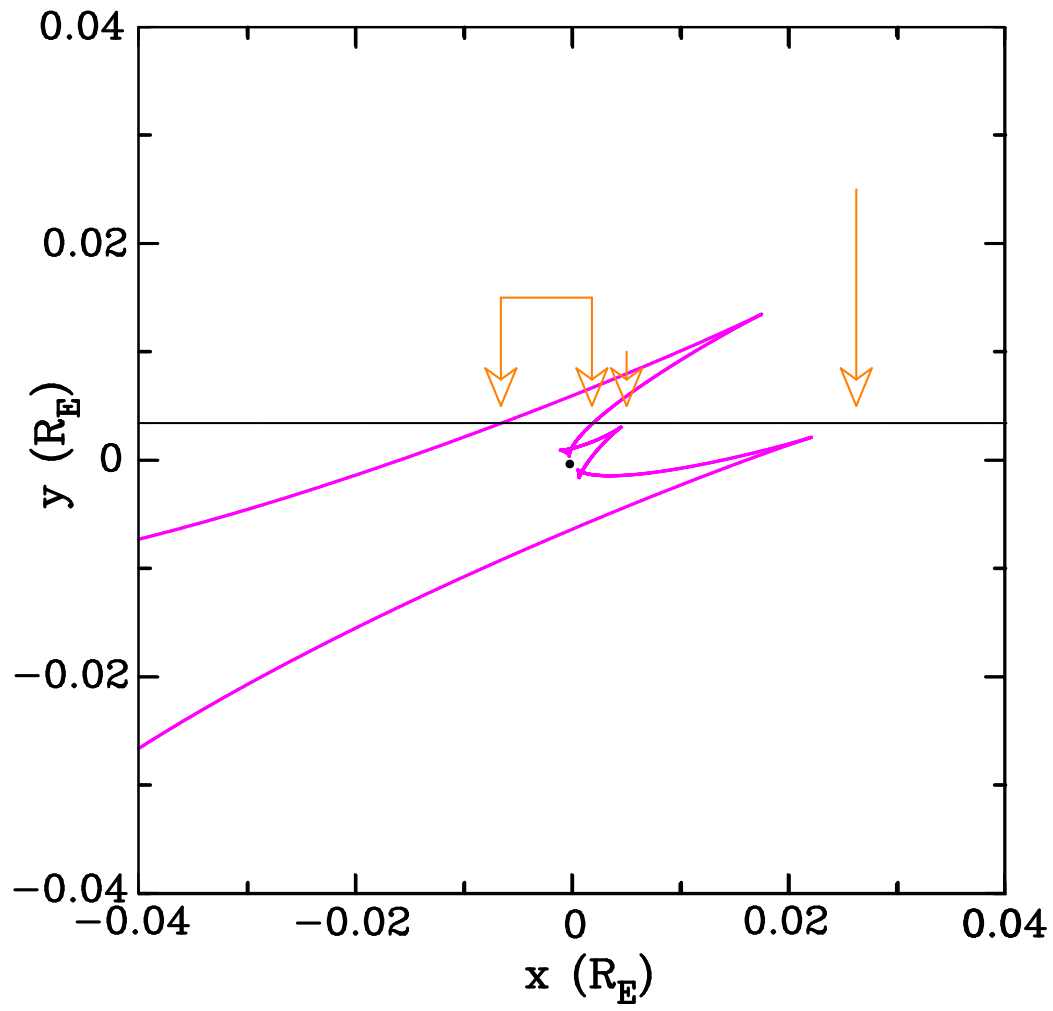

Figure 4.3: Close up of the event shown in Figure 4.2, with the primary lens in black, the caustic curves in magenta, and the source track in black. 


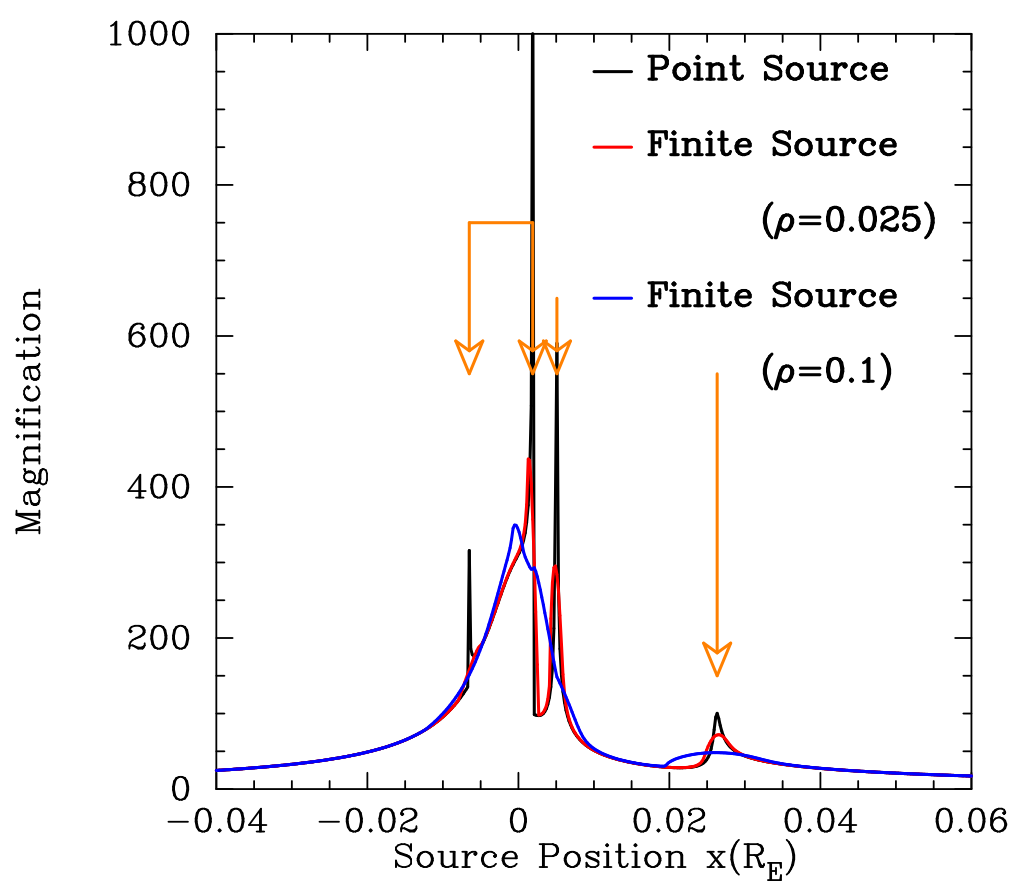

Figure 4.4: The magnification over a range of source positions for a triple lens event showing the point source (black), a finite source disk with a radius $0.005 R_{E}$ (red), and a finite source disk with a radius $0.02 R_{E}$ (blue).

\subsection{Limb Darkening}

Limb darkening is the effect where the intensity of the source disk is not uniform across the source disk, but is darker at the edge (limb) of a source. This is occurs because the central core of the star is much hotter than the outer layer of the star. Therefore, from the Stefan-Boltzmann law, more photons are emitted from the centre of the source than the outer edge of the source. We give a brief discussion and derivation of limb darkening expression here, with the full derivation given in Appendix D, Section D.3.

When an observer is some distance away from a spherical source, the observed intensity $I(\psi)$ of a limb darkened star can be approximated by,

$$
I(\psi)=I_{0} \sum_{k=0}^{N} a_{k} \cos ^{k}(\psi),
$$

where $\sum_{k=0}^{N} a_{k}=1, I_{0}=I(0)$ is the intensity at the centre of the source disk, and $\psi$ is the angle of incidence, as shown in Figure 4.5. The limb darkening effect can be modelled in the simplest case by the linear model, with $a_{0}=1-u$ and $a_{1}=u$, where $0 \leq u \leq 1$. From Equation 4.2, it can be shown that if $\rho$ is the 


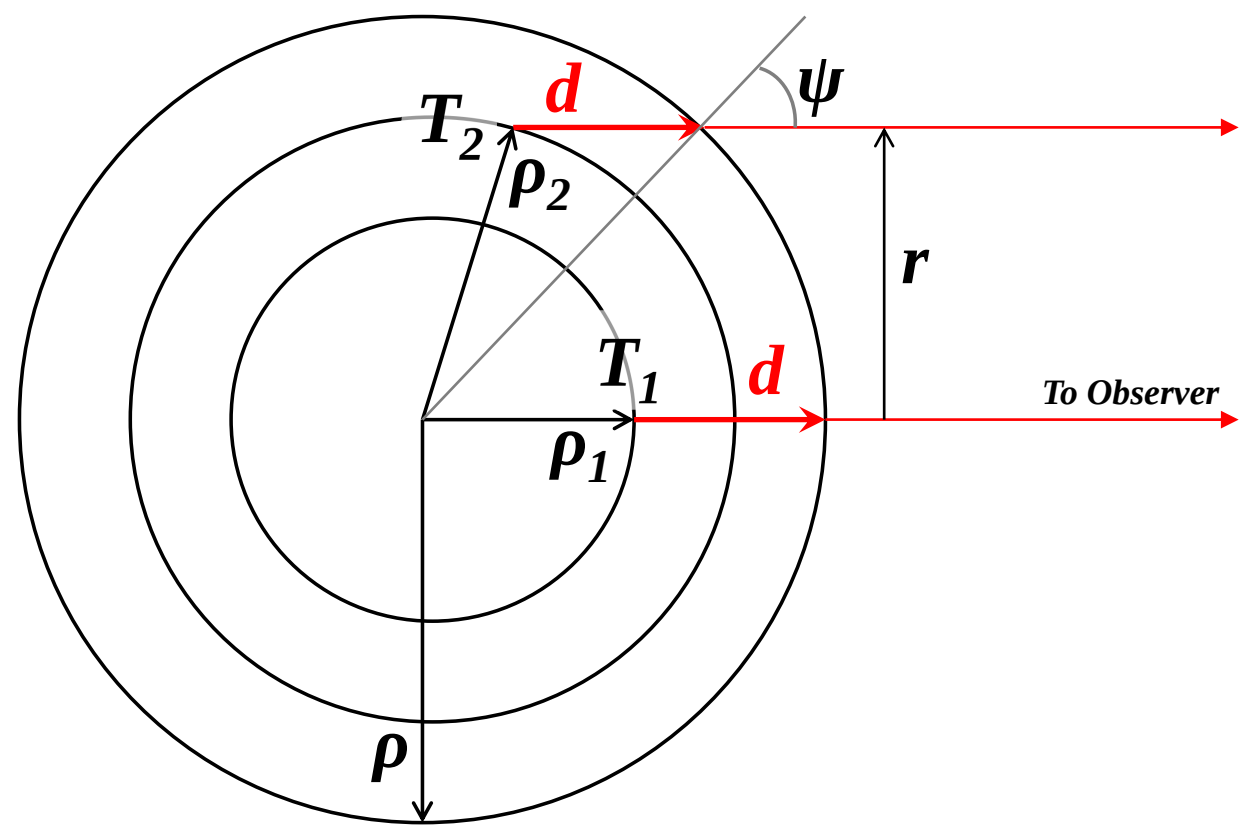

Figure 4.5: The observer receives photons emitted along the line of sight from a distance $d$ within a star. For photons near the edge of the source disk, these photons are emitted from a shallower (and therefore cooler) depth, at a radius $\rho_{2}$, than the photons from the centre of the disk.

radius of the source disk and $r$ is the radial distance from the centre of the source disk, then the limb darkened intensity across a source disk can be given by,

$$
\frac{I(r)}{I_{0}}=1-u\left(1-\sqrt{1-\left(\frac{r}{\rho}\right)^{2}}\right) .
$$

As discussed previously, to calculate the magnification of an image, we take the ratio of the image area to the source area. We can define $I_{a v e}$ as the average intensity across the source disk, so that the total flux of the source can be expressed as $I_{\text {ave }} \pi \rho^{2}$. Therefore the expression for the limb darkening variation can be given in terms of this average intensity (Gould, 2008), given by,

$$
\frac{I(r)}{I_{\text {ave }}}=1-\Gamma\left(1-\frac{3}{2} \sqrt{1-\left(\frac{r}{\rho}\right)^{2}}\right),
$$

where $\Gamma$ is called the limb darkening coefficient with $0 \leq \Gamma \leq 1$. Figure 4.6 shows some profiles of the intensity of the source star plotted against the radial 
distance from the centre for limb darkening coefficients $\Gamma=0,0.2,0.4,0.6,0.8,1$. These profiles show that a source disk with a limb darkening coefficient $\Gamma=0$ does not have any variation in brightness across its surface.

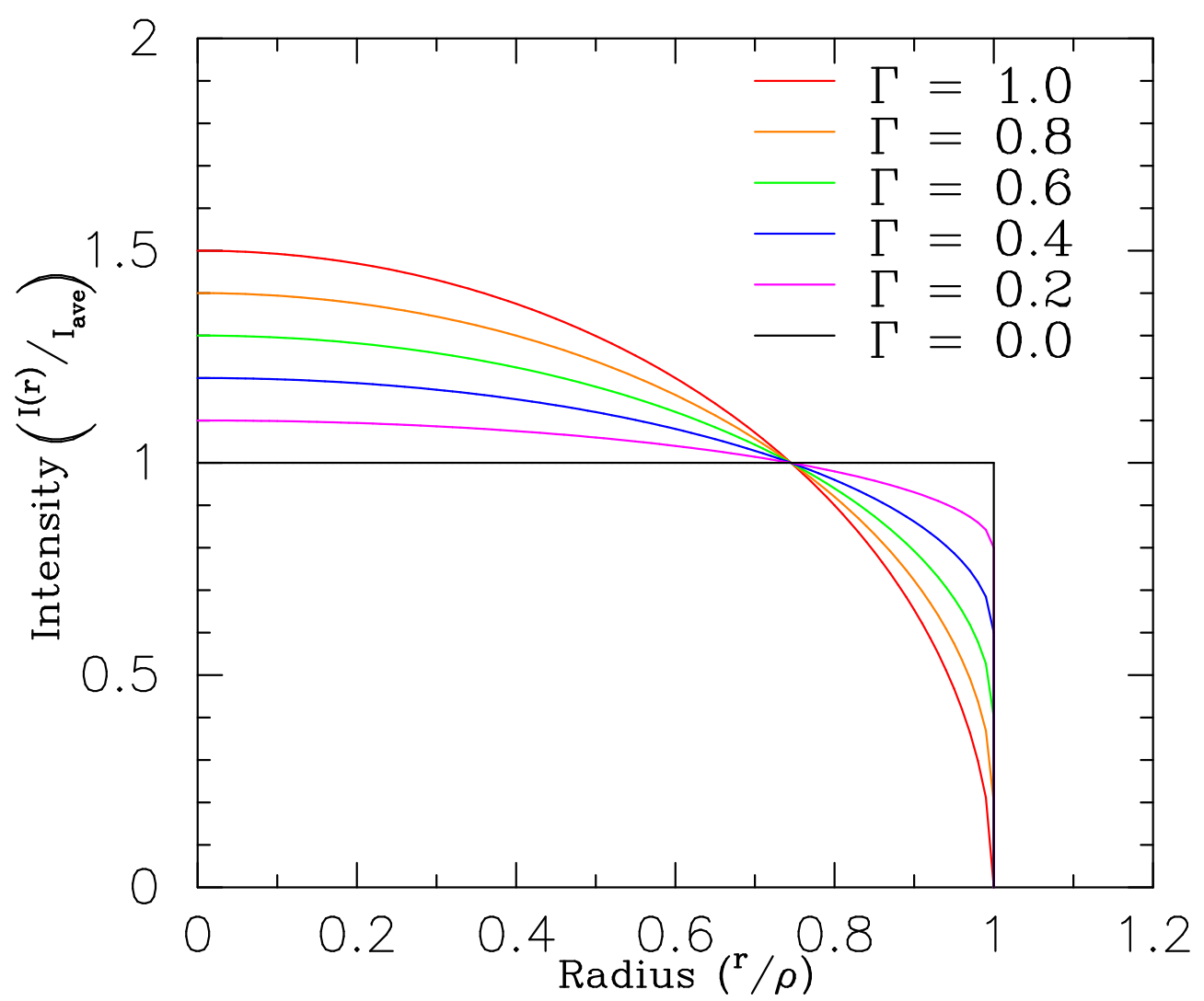

Figure 4.6: The intensity over the radial distance from the centre of the source disk for the limb darkening coefficients $\Gamma=0,0.2,0.4,0.6,0.8,1$ given by the black, red, orange, green, blue and purple lines respectively.

In microlensing, limb darkening becomes especially important in cases where a large source disk straddles a caustic curve. In these situation, one part of the image will be projected onto both sides of the critical curve, as shown in Figure 3.5. With limb darkening, the average intensity of each "part image" on either side of the caustic curve may vary from the average intensity of the source itself. Therefore, this variation of intensity across the source disk needs to be incorporated into methods that estimate the finite magnification of the source disk. 


\subsection{Polygon Method}

The polygon approximation is a method first derived from Stokes' Theorem (Gould and Gaucherel, 1997). This method estimates the area of the source disk by inscribing a polygon with $n$ vertices within the disk. The vertices on the source can then used to find the points on the image plane and these image points can be connected to form polygons inscribed within the images. These image polygons can then be used to estimate the area of the constituent finite images. The ratio of the area of each image polygon to the area of the source polygon gives an approximation for the magnification of each image.

\subsubsection{Area of a Polygon}

The area of a polygon on an xy-plane can be calculated using a variation of the trapezoidal rule used in numerical integration. The vertices of a polygon form a series of points $\left(x_{j}, y_{j}\right)$ where the areas between each edge of the polygon and the $\mathrm{x}$-axis is calculated using the area of a trapezium given in Equation 4.5. These areas are then added together to give the total area of a polygon expressed by,

$$
\text { Area }_{\text {trapezium }}=\left(\mathrm{x}_{\mathrm{j}-1}-\mathrm{x}_{\mathrm{j}}\right) \frac{\mathrm{y}_{\mathrm{j}-1}+\mathrm{y}_{\mathrm{j}}}{2} \text {. }
$$

Figure 4.7 shows the area of the 5 -sided polygon, which is given by,

$$
\text { Area }_{\text {polygon }}=\text { Area }_{0,1}+\text { Area }_{1,2}+\text { Area }_{2,3}+\text { Area }_{3,4}+\text { Area }_{4,0} .
$$

In Figure 4.7, the areas of the red shapes are negative. Area ${ }_{4,0}$ is negative because it is below the $\mathrm{x}$-axis and Area $_{2,3}$ is negative because $x_{3}<x_{2}$. Area An, $_{3,4}$ is below the $\mathrm{x}$-axis but $x_{4}<x_{3}$, so 4.6 returns a positive area for this triangle.

For two adjacent trapeziums defined by the two pairs of vertices $\left(x_{j-1}, y_{j-1}\right),\left(x_{j}, y_{j}\right)$ and $\left(x_{j}, y_{j}\right),\left(x_{j+1}, y_{j+1}\right)$, the formula for their respective areas can be given as,

$$
\begin{aligned}
\text { Area }_{\mathrm{j}-1, \mathrm{j}} & =\frac{1}{2}\left(x_{j-1} y_{j}-x_{j} y_{j-1}\right)+\frac{x_{j-1} y_{j-1}}{2}-\frac{x_{j} y_{j}}{2} \\
\text { Area }_{\mathrm{j}, \mathrm{j}+1} & =\frac{1}{2}\left(x_{j} y_{j+1}-x_{j+1} y_{j}\right)+\frac{x_{j} y_{j}}{2}-\frac{x_{j+1} y_{j+1}}{2} .
\end{aligned}
$$



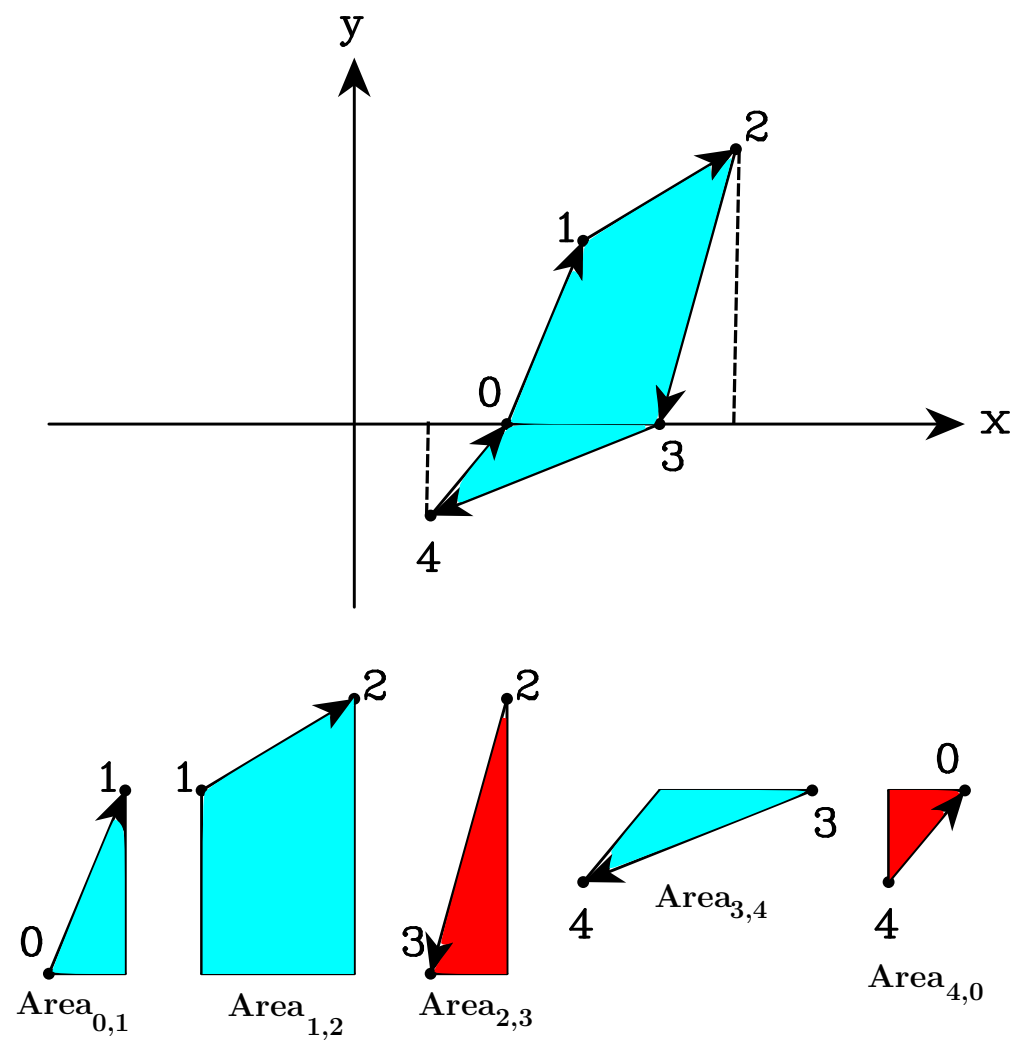

Figure 4.7: The area of a polygon, which represents the image, and the trapeziums and triangles that make up the shape of the image.

When the trapezium areas Area $\mathrm{j}_{-1, \mathrm{j}}$ and Area $_{\mathrm{j}, \mathrm{j}+1}$ are added together, their third and second terms respectively cancel. By adding together the areas from all the trapeziums, the second and third terms for all the trapezium areas cancel. Therefore, the area of a polygon with $n$ vertices can be given as,

$$
\text { Area }_{\text {polygon }}=\frac{1}{2} \sum_{\mathrm{j}=1}^{\mathrm{n}}\left(\mathrm{x}_{\mathrm{j}-1} \mathrm{y}_{\mathrm{j}}-\mathrm{x}_{\mathrm{j}} \mathrm{y}_{\mathrm{j}-1}\right),
$$

where $\left(x_{0}, y_{0}\right)=\left(x_{n}, y_{n}\right)$.

As discussed in the Sections 3.1 and 3.2, the images on the same side of the critical curves as the lenses have a negative parity, while the images on the other side have a positive parity. Suppose the area of the source disk is calculated by going around the source polygon in a clockwise direction. The vertices for the polygons representing the images with positive parity will also be sequenced in the clockwise direction, returning a positive area. However, the vertices for the polygons representing the images with negative parity will be sequenced in the anticlockwise direction, giving a negative value for the area. Therefore these 
areas need to be multiplied by their respective parities to get the absolute image magnifications.

When a source crosses a caustic curve, this method gets more complicated, as shown in Figure 3.5. When this occurs, part of the image is formed on one side of the critical curves, while the other part of the image is on the other side of the critical curves. In this case, the two parts of the image need to be connected to form a single polygon. However, this can only be achieved if the vertices are sequenced in the same direction (either both clockwise or both anticlockwise), so the sequence for one part of this image needs to be reversed before the image parts are joined (see Chote (2011)).

If the image vertices are represented on the xy-plane, and the source vertices are represented on the uv-plane, the total source magnification without the effects of limb-darkening can be expressed as,

$$
A=\frac{\sum_{\text {images }}\left|\sum_{j=1}^{n}\left(x_{j-1} y_{j}-x_{j} y_{j-1}\right)\right|}{\sum_{j=1}^{n}\left(u_{j-1} v_{j}-u_{j} v_{j-1}\right)}
$$

where $\sum_{\text {images }}$ represents the summation of all the (physical) image magnifications.

\subsubsection{Constructing Polygon}

To construct a polygon that accurately represents the finite source disk with a radius $\rho$ and its corresponding images, it is important to consider the number of vertices in the polygon, and the spacing between the vertices. Supposing the source disk is represented by a regular polygon with $n$ vertices, then the angle of vertex $j$ from the $\mathrm{x}$-axis about the centre of the source disk can be given by,

$$
\theta_{j}=\frac{2 \pi j}{n}
$$

While this places the vertices on the source disk at regular intervals, when these vertices are mapped onto the image plane, they are positioned at highly irregular intervals about the images. This is shown in the left panel of Figure 4.8 the right panel of this diagram shows the separation between the red and blue 
vertices on the positive parity image as the source moves along the horizontal axis. This demonstrates that, as the source disk approaches the lens, the spacing between the vertices increases rapidly. This could potentially lead to numerical inaccuracies when calculating the area of the images.
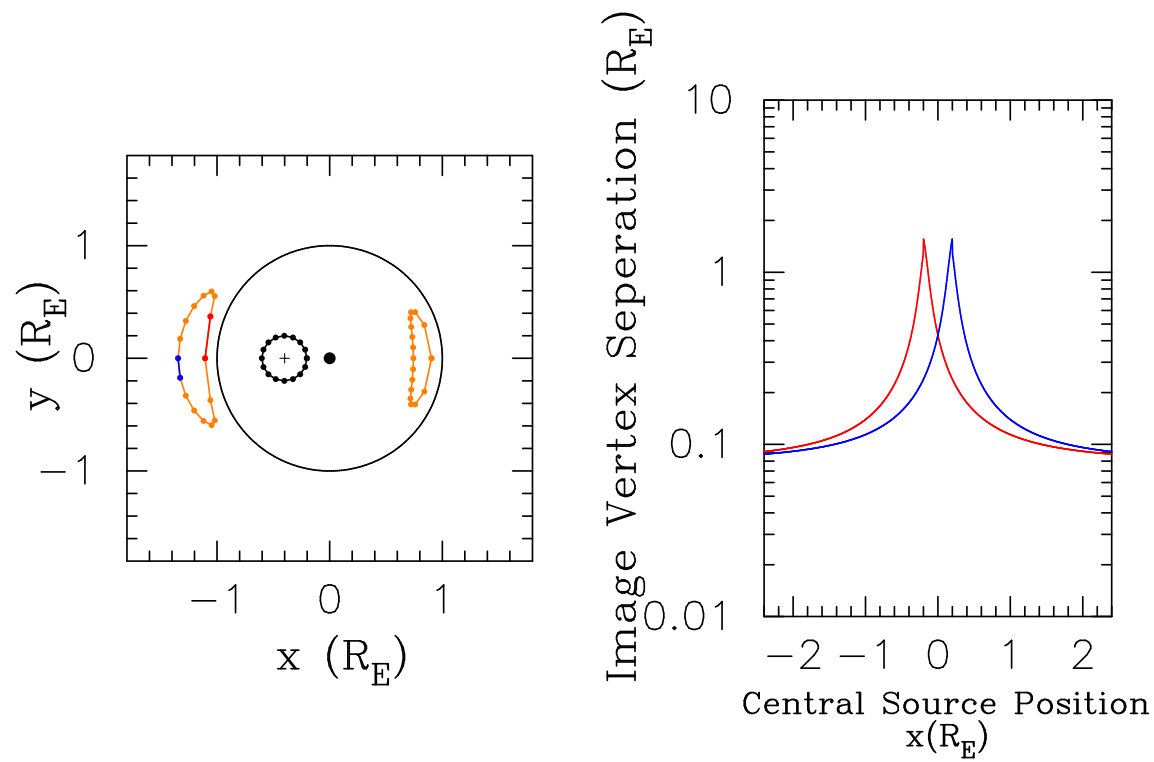

Figure 4.8: The left panel shows the shape of the source and images when the source is represented by a regular polygon. The right panel shows the separation between the pairs of vertices of the corresponding coloured spacing as the source moves along the horizontal axis.

A better approach is to place the vertices dynamically so the spacing between adjacent vertices on the images is roughly constant. One such dynamic method was developed by Chote (2011). This method defines $\Delta \theta_{i+1}=\theta_{i+1}-\theta_{i}$ as the angular spacing between any two vertices, at the angular positions $\theta_{i+1}$ and $\theta_{i}$ around the circumference of the source disk.

If $n_{\min }$ is defined as the minimum acceptable number of vertices on the polygon and the initial vertex is positioned at $\theta_{0}=0$, then the first angular spacing $\Delta \theta_{1}=\theta_{1}-\theta_{0}$ is defined as,

$$
\Delta \theta_{0}=\Delta \theta_{\max }=\frac{2 \pi}{n_{\min }} .
$$

If $\Delta z_{i}$ is the maximum distance between the vertex $i$ and vertex $i-1$ in any image, then the next angular spacing $\Delta \theta_{i+1}$ can be defined as,

$$
\Delta \theta_{i+1}=\min \left(\Delta \theta_{\max }, 0.75 \Delta \theta_{i} \frac{\Delta z_{i}}{\Delta z_{\max }}\right)
$$


where $\Delta z_{\max }$ is the maximum allowed distance between any two vertices. If the distance between the vertices does exceed $\Delta z_{\max }$, then the current vertices are deleted and angular spacing is halved: $\Delta \theta_{i+1} / 2 \rightarrow \Delta \theta_{i+1}$. This approach is demonstrated in Figure 4.9.
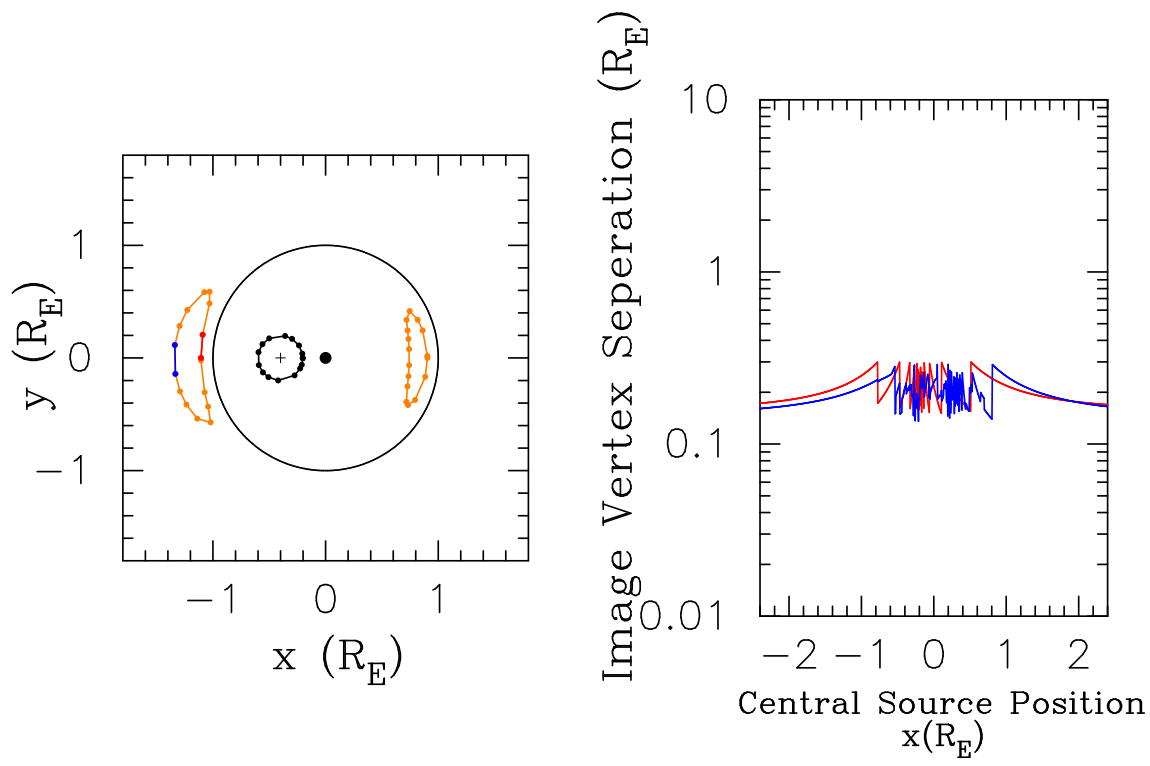

Figure 4.9: The left panel shows the shape of the source and images when the vertices around the source a positioned using the dynamic method developed by Chote (2011). The right panel shows the separation between the pairs of vertices of the corresponding coloured spacing as the source moves along the horizontal axis.

Since the point source magnification gives an approximate ratio of the sum of the image areas to the source area, a more 'natural' dynamic approach was developed as part of this research. This approach expresses the dynamic spacing at the point $\left(u_{j}, v_{j}\right)$ in terms of the point source magnification $A_{j}$. To keep the spacing between the vertices on the images constant, the angular spacing of the vertices on the source can be given by,

$$
\Delta \theta_{j}=\frac{\Delta \theta_{\max }}{A_{j}}
$$

Figure 4.10 shows this 'natural' dynamic spacing for a single lens event, showing the spacing between the vertices on either side of the positive parity image staying roughly the same when plotted against the distance between the lens and the source.

This 'natural' dynamic method becomes problematic when the source is close to the lens (or caustic curves in a multiple lens case). When the point source is 

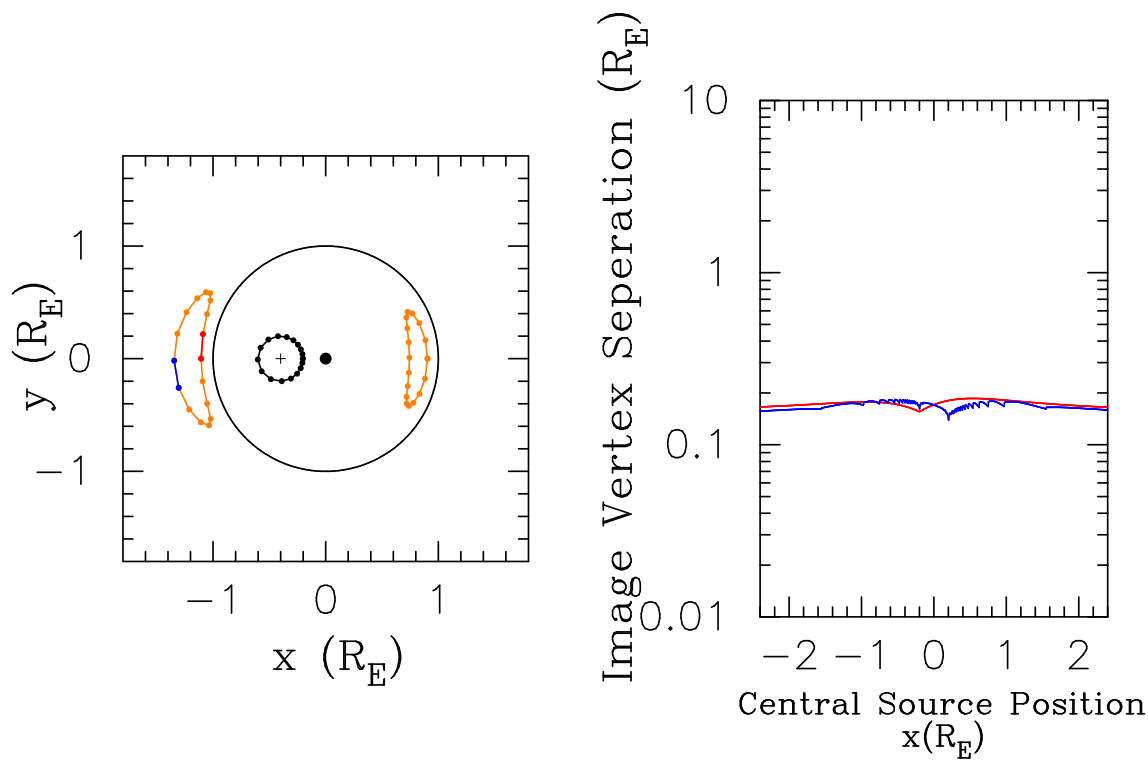

Figure 4.10: The left panel shows the shape of the source and images when the vertices around the source a positioned using the 'natural' dynamic method. The right panel shows the separation between the pairs of vertices of the corresponding colour as the source moves along the horizontal axis.

near to the single lens, the point source magnification grows approximately as the inverse of its distance from the lens. Therefore, even when a small source is $0.1 R_{E}$ from the lens, it is using roughly 10 times as many points as when it was far $\left(>1 R_{E}\right)$ from the source, and roughly 100 times as many when it is $0.01 R_{E}$ from the lens. From numerical investigations, we found that polygons with no less than 16 vertices were required to give an accurate calculation for the source magnification when the source disk was far from the lens. Therefore, using the 'natural' dynamic method, we found that polygons produced by the dynamic method gave more than 1500 vertices for sources $0.01 R_{E}$ from the lens.

For this reason, we concluded that Chote's dynamic method was more efficient for calculating the polygon areas as it constructs polygons using fewer vertices than the 'natural' dynamic method.

\subsubsection{Limb Darkening}

The polygon approximation by itself assumes a source of constant brightness with no limb darkening. However, the effects of limb darkening can be included in this approximation by adding concentric circles inside the circularly symmetric source, breaking the source disk up into $N_{R}$ annuli, as proposed by Chote (2011). The 
limb darkening effects can then be approximated by assuming each annulus has a constant intensity. The intensity of the $k^{\text {th }}$ annulus can be given by Equation 4.4, where $r=r_{k-\frac{1}{2}}$, which is the radius midway between the inner and outer radii $r_{k-1}$ and $r_{k}$ respectively, bounding this annulus. Polygons can then be inscribed inside each of the concentric circles to give a series of nested polygons. The area of a particular annulus, bounded by an inner and outer circle, can be approximated by the difference between the areas of its respective inner and outer polygons. These areas are then multiplied by their respective limb darkening intensity and are summed together to give the total flux from the source.

The vertices of the nested polygons on the source disk can then be mapped to points on the image plane. These points on the image plane describe the vertices of a series of nested polygons inside each image, corresponding to the annuli in the source disk. In a similar way, the area of each annuli is approximated by the difference between the areas of the inner polygon, with an area of Area $\mathrm{k}_{-1}$, and the outer polygon, with an area of $A_{r e a}$, and the flux, $F$, of each image can be expressed by,

$$
F=\sum_{k=1}^{N_{R}} I\left(r_{k-\frac{1}{2}}\right)\left(\text { Area }_{\mathrm{k}}-\text { Area }_{\mathrm{k}-1}\right)
$$

where $I\left(r_{k-\frac{1}{2}}\right)$ is the limb darkening intensity for the corresponding annuli. The magnification, $\mu$, of each image is then calculated as,

$$
\mu=\frac{F}{F_{S}}
$$

where $F_{S}$ is the corresponding flux calculation for the source.

Chote proposed that the radii of the concentric circles can be found using two methods. The first method is where the radii of the circles could be given at equal intervals, so that each annulus has the same width. In this case, the radius $r_{k}$ of the $k^{\text {th }}$ concentric circle would be given by $r_{k}=\frac{k}{N_{R}} \rho$, where $N_{R}$ is the number of concentric circles.

The second method is to define the radii so that the intensities of the concentric circles are at equal intervals. This can be achieved by inverting Equation 
4.4 to give the radius $r$ in terms of the intensity, $I$, at that radius $r_{k}$,

$$
\frac{r_{k}}{\rho}=\sqrt{1-\frac{4}{9 \Gamma^{2}}\left(\frac{I_{k}}{I_{a v e}}+\Gamma-1\right)^{2}},
$$

where the intensity of the $k^{t h}$ annulus is given by,

$$
I_{k}=I(0)+\frac{k}{N_{R}}(I(\rho)-I(0)) .
$$

The profiles of the sizes and intensities of the annuli using these two methods are shown in Figure 4.11. The left panel shows the first method where the radii of the circles are given at equal intervals and the right panel shows the second method where the intensities of the concentric circles are at equal intervals.
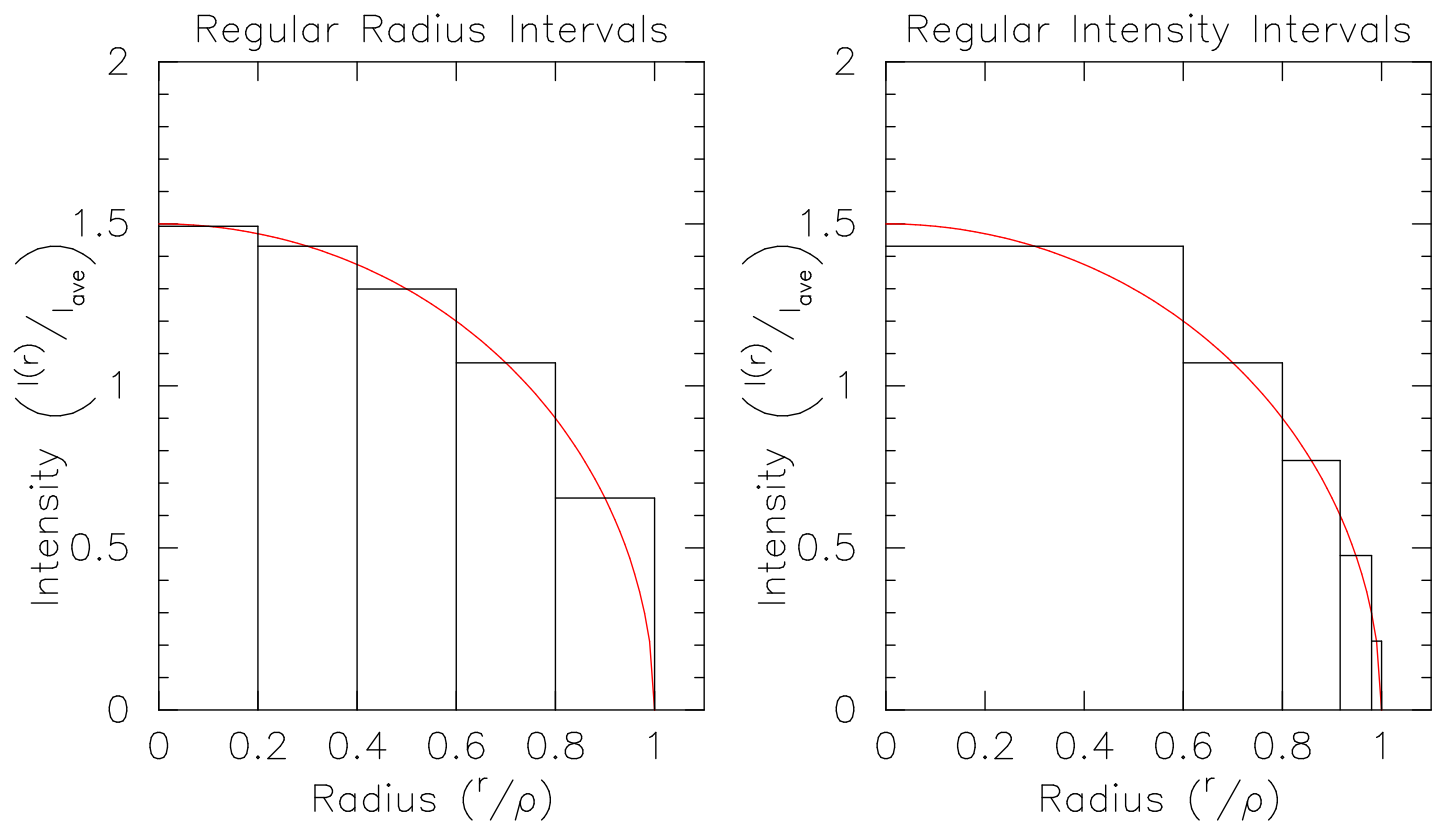

Figure 4.11: The two profiles for the annuli that make up the limb-darkened source disk. The left panel shows annuli where the radii of the circles are given at equal intervals and the right panel shows annuli where the intensities of the concentric circles are at equal intervals.

\subsection{Multipole Approximations}

While the polygon method provides a reasonably accurate approximation for the image magnifications, it requires the calculation of many image positions, for the approximation to reach an adequate level of accuracy. The number of points is multiplied even further when one includes the effects of limb darkening. 
Every image point found increases the amount of computing time required to calculate the image magnifications, which means the polygon method is clearly very computationally expensive.

To include the finite source effects while still remaining computationally economic, Gould (2008) developed the hexadecapole approximation which uses 13 points on the source disk to estimate the magnification of the source. This approximation uses the Taylor series to approximate the source magnification across the source disk and incorporates the effects of limb darkening. Using the derivation proposed by Gould, we derive a slightly different hexadecapole approximation from first principles. We also introduce the quadrapole approximation, which approximates the finite source magnification using 5 points on the source disk. The quadrapole approximation can be viewed as an approximation that is faster than the hexadecapole method but more accurate the point source approximation. A description and derivation of these approximations is given here, with the full derivation of these approximations can be found in Appendix D.

\subsubsection{Derivation of Multipole Approximations}

To derive the quadrapole and hexadecapole approximations, we first consider a finite circular source disk of radius $\rho$. Since limb darkening depends only on radius, the intensity profile of the source disk is circularly symmetric about the centre of the disk. We can exploit this symmetry to make the derivation of the multipole approximations easier. If $\left(u_{0}, v_{0}\right)$ is the point at the centre of the source disk in Cartesian coordinates, then a point on the source disk can be represented by the point $(r, \theta)$ in polar coordinates, or $(u, v)=\left(u_{0}+r \cos (\theta), v_{0}+r \sin (\theta)\right)$ in Cartesian coordinates. The point source magnification at this point can be given as $A(r, \theta)$ or $A(u, v)$.

To calculate the source magnification, we can define $I_{\text {ave }}$ as the average intensity over the disk. The total brightness of the source can then be calculated by integrating across the area of the source disk, given as,

$$
A_{\text {disk }}=\frac{\int_{\text {disk }} A(r, \theta) I(r) d a}{\int_{\text {disk }} I(r) d a}=\frac{2}{\rho^{2} I_{\text {ave }}} \int_{0}^{\rho} A(r) I(r) r d r,
$$


where,

$$
A(r)=\frac{1}{2 \pi} \int_{0}^{2 \pi} A(r, \theta) d \theta
$$

If the disk is uniform in brightness (no limb darkening), so that $I(r)=I_{\text {ave }}$, then it can be shown that the magnification of the finite source disk can be given by $A_{\text {disk }}=\int_{0}^{\rho} A(r) r d r$.

For any point $(u, v)$ on the source disk, a 2-dimensional Taylor series can be used to estimate the source magnification $A(u, v)$ at that point. This Taylor series contains partial derivatives with respect to $u$ and $v$, but due to the circular symmetry of the source disk, any partial derivative with an odd order with respect to $u$ or $v$ cancels to zero. Therefore, the Taylor series simplifies to,

$$
A(r)=A_{0}+A_{2} r^{2}+A_{4} r^{4}+\cdots
$$

where,

$$
\begin{aligned}
& A_{0}=A_{00} \\
& A_{2}=\frac{1}{4}\left[\frac{\partial^{2} A\left(u_{0}, v_{0}\right)}{\partial u^{2}}+\frac{\partial^{2} A\left(u_{0}, v_{0}\right)}{\partial v^{2}}\right] \\
& A_{4}=\frac{1}{64}\left[\frac{\partial^{2} A\left(u_{0}, v_{0}\right)}{\partial u^{4}}+2 \frac{\partial^{2} A\left(u_{0}, v_{0}\right)}{\partial u^{2} \partial v^{2}}+\frac{\partial^{2} A\left(u_{0}, v_{0}\right)}{\partial y^{4}}\right] .
\end{aligned}
$$

The quadrapole approximates the finite source disk magnification using the Taylor series up to the term $A_{2}$, estimating the magnification of the finite source disk as,

$$
A_{\text {quadrapole }}=A_{0}+\frac{A_{2} \rho^{2}}{2}\left(1-\Gamma \frac{1}{5}\right) .
$$

The hexadecapole approximates the finite source disk magnification using the Taylor series up to the higher term, $A_{4}$, giving a more accurate estimation for the finite source disk magnification, given as,

$$
A_{\text {hexadecapole }}=A_{0}+\frac{A_{2} \rho^{2}}{2}\left(1-\Gamma \frac{1}{5}\right)+\frac{A_{4} \rho^{4}}{3}\left(1-\Gamma \frac{11}{35}\right) .
$$

The accuracy of the estimated finite source disk magnification depends partly on the accuracy of the derivatives used in the Taylor expansion. These derivatives can be calculated using two different methods. The first method is to find 
an analytical expression for these derivatives at the point $\left(u_{0}, v_{0}\right)$ by differentiating the inverse of the Jacobian determinant (point source magnification). The second method is to estimate the derivative by numerical methods. The former method calculates the multipole approximations using only a single point on the source, and is known hereafter as the single-point multipole approximation. The latter method uses the point source magnifications from several points around the source disk to approximate the average derivatives across the whole disk, and is known hereafter as the multiple-point multipole approximation.

\subsubsection{Single-Point Multipole Approximations}

The Jacobian given at the beginning of Chapter 3 is expressed in terms of the complex number $z=x+i y$ and its conjugate $\bar{z}=x-i y$. Therefore, it is easier to find the analytical expressions for the derivatives, if the derivatives given in Equations 4.22 - 4.24 are converted from derivatives in terms of $x$ and $y$, into derivatives in terms of $z$ and $\bar{z}$. In this case, Equations $4.22-4.24$ can be given by,

$$
\begin{aligned}
& A_{0}=A, \\
& A_{2}=\sum_{\text {images }} \frac{1}{J} \frac{\partial^{2} \mu}{\partial z \partial \bar{z}} \\
& A_{4}=\sum_{\text {images }} \frac{1}{4 J^{2}} \frac{\partial^{4} \mu}{\partial z^{2} \partial \bar{z}^{2}} .
\end{aligned}
$$

The expressions for the second and fourth derivatives can be obtained using Faà di Bruno's formula (Faà di Bruno, 1855). The expression for the second derivative is given by,

$$
\frac{\partial^{2} \mu}{\partial z \partial \bar{z}}=\frac{\partial \mu}{\partial J} \frac{\partial^{2} J}{\partial z \bar{z}}+\frac{\partial^{2} \mu}{\partial J^{2}} \frac{\partial J}{\partial z} \frac{\partial J}{\partial \bar{z}}
$$

while the expression for the fourth derivative can be found in Appendix B, Equation B.23.

If the sign of the Jacobian determinant is given by $\operatorname{sgn}(\mathrm{J})$, then the $k^{\text {th }}$ order derivative for the source magnification can be given by,

$$
\frac{\partial^{k} \mu}{\partial J^{k}}=\sum_{\text {images }} \frac{(-1)^{k} k ! \operatorname{sgn}(J)}{J^{k+1}} .
$$


Using the expression for the Jacobian from the previous chapter, it can be shown that the derivatives of the Jacobian with respect to $z$ and $\bar{z}$ can be given by,

$$
\frac{\partial^{p+q} J}{\partial z^{p} \partial \bar{z}^{q}}=(-1)^{p+q+1}\left(\sum_{j=0}^{N} \frac{(p+1) ! \epsilon_{j}}{\left(z-r_{j}\right)^{p+2}}\right)\left(\sum_{j=0}^{N} \frac{(q+1) ! \epsilon_{j}}{\left(\bar{z}-\bar{r}_{j}\right)^{q+2}}\right) .
$$

Equations 4.27 - 4.32, can be combined to give expressions for $A_{2}$ and $A_{4}$. These expressions, along with the source radius, $\rho$, and the limb darkening coefficient, $\Gamma$, can be used to estimate the magnification of the finite source disk using the quadrapole and hexadecapole approximation.

While these single-point multipole approximations account for the effects of limb darkening, they still suffer from some of the same problems that affected the point source approximation. For example, the magnification of the source calculated using the quadrapole and hexadecapole approximations still diverges as the source crosses a caustic curve.

\subsubsection{Multiple-Point Multipole Approximations}

The second method to estimate the derivatives is to use numerical approximations. This is done by obtaining point source magnifications for several positions on the source disk, and using these points to approximate the derivatives over the source disk. These numerical derivatives can be derived from Lagrange polynomials.

Given a $f(x)$ and a set of $k+1$ distinct points $\left(x_{1}, f\left(x_{1}\right)\right), \cdots,\left(x_{k+1}, f\left(x_{k+1}\right)\right)$, the Lagrange polynomial $p(x)$ is the unique polynomial of degree $k$ that can be interpolated from these points. This polynomial can be differentiated up to the $k^{\text {th }}$ order derivative, and these derivatives of the polynomial $p(x)$ describe approximate derivatives for the function $f(x)$. Deriving these approximate numerical derivatives from Lagrange polynomial is not covered in this thesis, however, it is important to note that estimating a derivative of $f(x)$ to order $k$ requires no less than $k+1$ distinct points.

If we have more distinct points, we can interpolate a polynomial with a higher degree, and hence obtain a more accurate approximation of the function $f(x)$. 
Given the points $\left(x_{1}, y_{1}\right),\left(x_{2}, y_{2}\right), \ldots\left(x_{k}, y_{k}\right)$ on an xy-plane, a unique Lagrange polynomial of degree $k-1$ can be created that passes through all $k$ points. By obtaining the expression for the Lagrange polynomial and differentiating this expression, we can derive formulae approximating the derivatives up to order $k-1$, for a function going through the $k$ points.

In the quadrapole approximation, the second derivative terms in the expression for $A_{2}$ can be obtained using three points along the line $v=v_{0}$, and three points along the line $u=u_{0}$. The centre points of the two sets of collinear points are both at the position $\left(u_{0}, v_{0}\right)$, and the remaining four points are all at a distance $\rho$ from this central point. These five points can then be used to obtain estimates for the second derivatives $A_{u u}\left(u_{0}, v_{0}\right)$ and $A_{v v}\left(u_{0}, v_{0}\right)$ (Abramowitz and Stegun, 1965). These second derivatives can be used to find an expression for $A_{2} \rho^{2}$ in the Equation 4.25. The expression for this term is given by,

$$
\begin{gathered}
A_{2} \rho_{\text {quadrapole }}^{2}=\frac{1}{4}\left[A\left(u_{0}+\rho, v_{0}\right)+A\left(u_{0}-\rho, v_{0}\right)\right. \\
\left.+A\left(u_{0}, v_{0}+\rho\right)+A\left(u_{0}, v_{0}-\rho\right)\right] \\
-A\left(u_{0}, v_{0}\right) .
\end{gathered}
$$

In the hexadecapole approximation, the fourth derivatives $A_{\text {uиuи }}\left(u_{0}, v_{0}\right)$ and $A_{v v v v}\left(u_{0}, v_{0}\right)$ can be derived from two 4 th degree Lagrange polynomials obtained by no less than 5 points along the lines $v=v_{0}$ and $u=u_{0}$ respectively. Each of these derivatives use a point located at $\left(u_{0}, v_{0}\right)$, along with four other points, two located at a distance $\rho$ from this central point, and two located at a distance $\frac{\rho}{2}$ from this central point. The hexadecapole approximation also requires four more points, located at $\left(u_{0} \pm \frac{\rho}{2}, v_{0} \pm \frac{\rho}{2}\right)$, in order to obtain the 'cross derivative' $A_{\text {uuvv }}\left(u_{0}, v_{0}\right)$.

With five points along the lines $v=v_{0}$ and $u=u_{0}$, the second derivatives $A_{u u}\left(u_{0}, v_{0}\right)$ and $A_{v v}\left(u_{0}, v_{0}\right)$ respectively can also be approximated using two 4 th degree Lagrange polynomials. This gives a much better approximation for the second derivative than the quadrapole approximation which estimates the second derivative using only 3 points along each line. Therefore, the expression for the 
terms $A_{2} \rho^{2}$ and $A_{4} \rho^{4}$ in Equation 4.26 can be given by the following formula,

$$
\begin{aligned}
A_{2} \rho_{\text {hexadecapole }}^{2} & =\frac{1}{3}\left[16 A_{\rho / 2,+}-A_{\rho,+}\right], \\
A_{4} \rho_{\text {hexadecapole }}^{4} & =2 A_{\rho / \sqrt{2}, \times}+A_{\rho,+}-8 A_{\rho / 2,+},
\end{aligned}
$$

where,

$$
A_{r,+}=\frac{1}{4} \sum_{j=0}^{3} A\left(u_{0}+r \cos \left(j \frac{\pi}{2}\right), v_{0}+r \sin \left(j \frac{\pi}{2}\right)\right)-A_{0}
$$

and,

$$
A_{r, \times}=\frac{1}{4} \sum_{j=0}^{3} A\left(u_{0}+r \cos \left(\frac{\pi}{4}+j \frac{\pi}{2}\right), v_{0}+r \sin \left(\frac{\pi}{4}+j \frac{\pi}{2}\right)\right)-A_{0}
$$

The hexadecapole expression derived by Gould (2008) uses four extra points on the edge of the source instead of the points $\left(u_{0} \pm \rho / 2, v_{0} \pm \rho / 2\right)$, and the term $A_{4} \rho^{4}$ is given by,

$$
A_{4} \rho_{\text {hexadecapole-Gould }}^{4}=\frac{A_{\rho,+}+A_{\rho, \times}}{2}-A_{2} \rho_{\text {hexadecapole }}^{2} .
$$

Figure 4.12 shows the 13 points used for the hexadecapole approximation, along with the source disk. The points used by both of the quadrapole and hexadecapole approximations are shown by the black dots 1-5, while the points used by only the hexadecapole method are shown by the dark grey dots 6-13. The hexadecapole developed by Gould uses the light grey dots instead of the points 10-13. The source disk is shown by the red circle.

Like the single-point multipole approximations, these multiple-point multipole approximations do not solve the problem of a diverging magnification as the source crosses a caustic. In fact, these numerical derivatives make the problem even worse, since there are more points representing the source, and the source magnification will diverge each time any of these points crosses a caustic.

Both the single-point and multiple-point multipole approximations attempt to find the magnification of the source using only a small number of discrete 


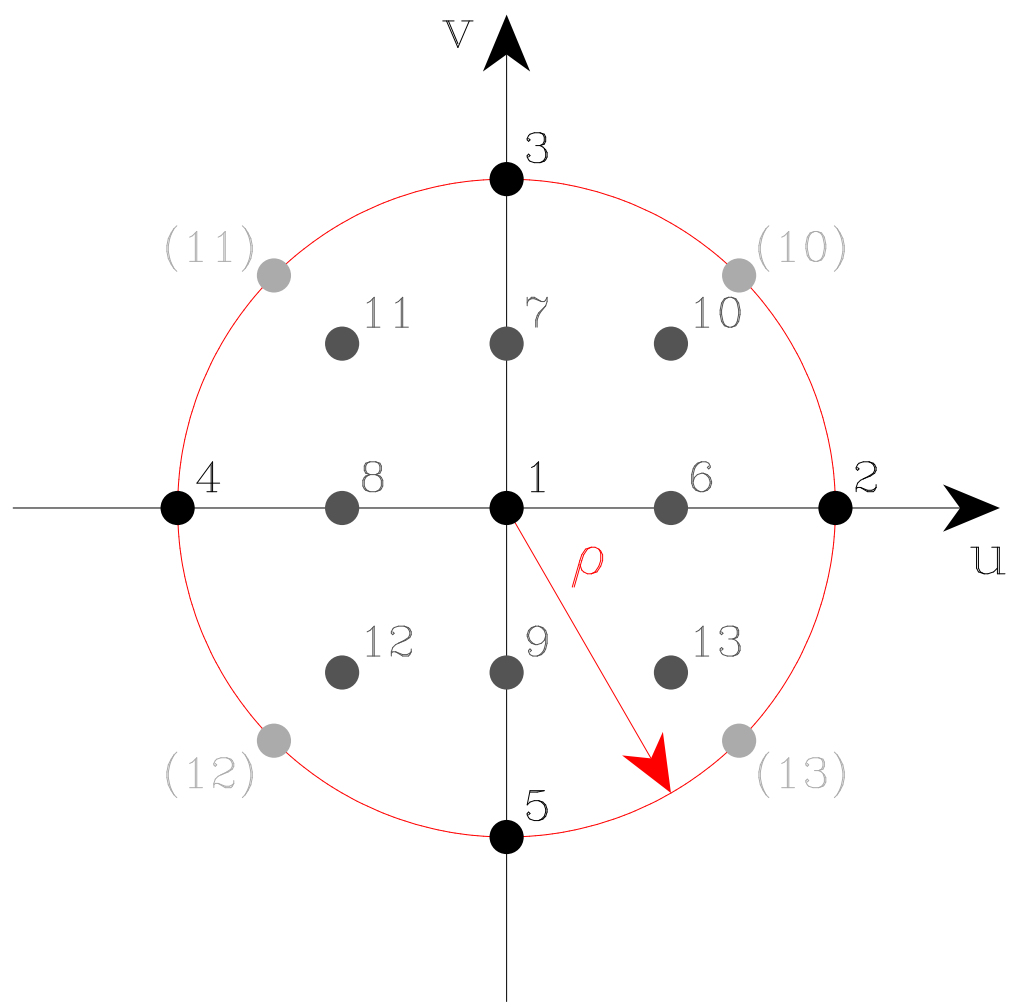

Figure 4.12: The positions of the point sources used in the multipole approximations, where $\rho$ is the radius of the source disk. The quadrapole approximation uses the black dots (1-5), and the hexadecapole approximation uses the black and grey dots (1-13).

points on the source disk. However the single-point multipole approximations attempt to calculate the magnification of the source disk by extrapolating from a single point at the centre of the source disk, while the multiple-point multipole approximations attempt to calculate this magnification by mostly interpolating from some selected points across the source disk. It has long been known that extrapolating data can more easily lead to large errors than interpolating, so the multiple-point multipole approximations are more likely to give an accurate estimate of the source magnification than their single-point equivalents.

It is known that a Taylor series using derivatives at a single point only converges across a finite disk. This disk of finite convergence is known as the radius of convergence. Outside the radius convergence, the Taylor series never converges, becoming more inaccurate as higher derivatives are included. In some cases, the radius of convergence may be smaller than the size of the source disk, which makes these derivatives at a point inappropriate to use in the Taylor series when estimating the finite source magnification. 
However, the derivatives calculated using numerical approximations are, by definition, the average derivatives across the source disk. Therefore, it seems more appropriate to use these numerical derivatives when estimating the magnification of the source.

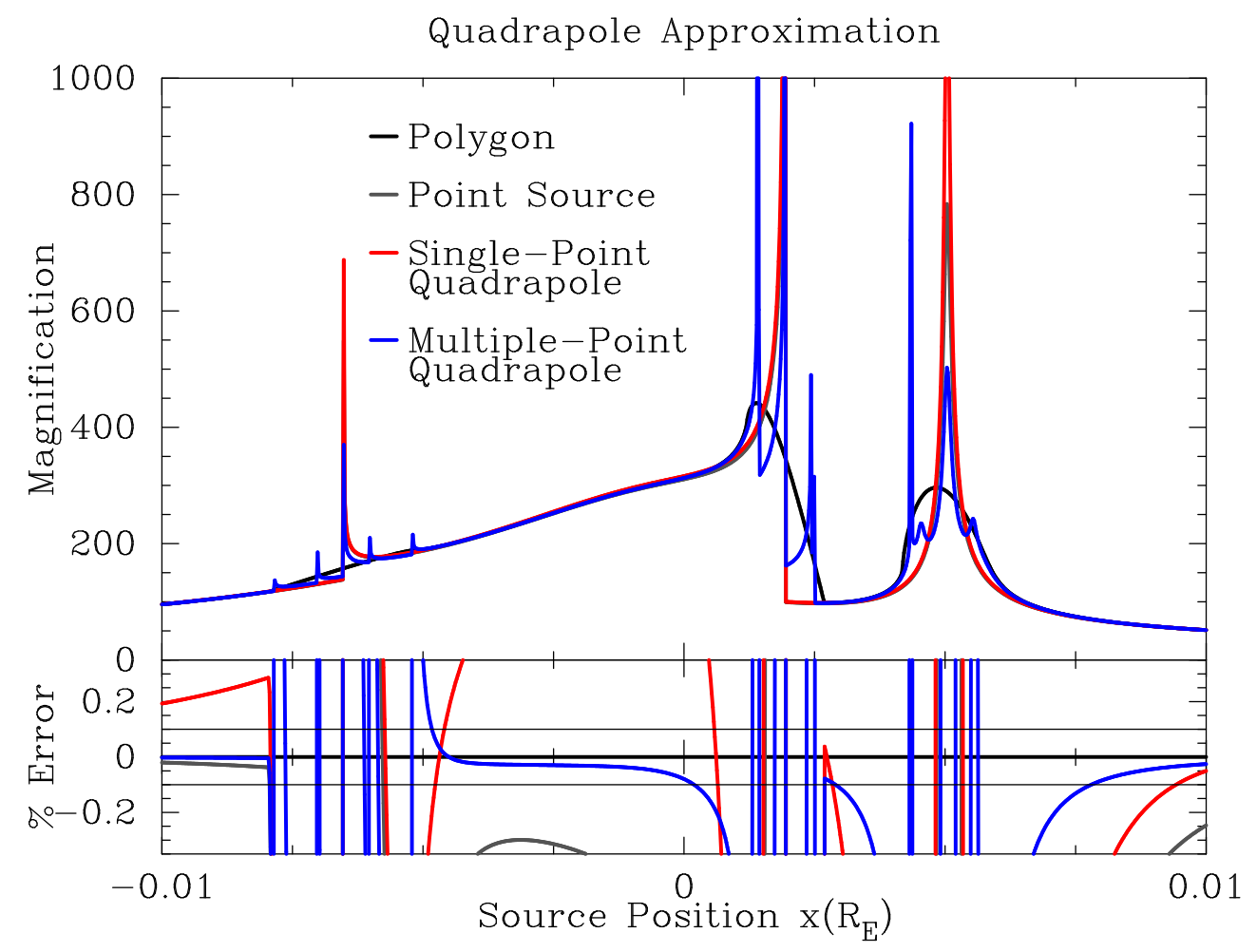

Figure 4.13: The lightcurves plotted using the single-point and multiple-point quadrapole approximations for a source radius of $0.0005 R_{E}$, for the same event shown in Figure 4.2.

Figures 4.13 and 4.14 show the lightcurves for the OGLE-2006-BLG-109 event for a source radius of $0.0005 R_{E}$ calculated using the quadrapole and hexadecapole approximations respectively. The lightcurves calculated using the single-point and multiple-point multipole approximations are shown by the red and blue curves respectively, and the point source and polygon methods are shown by the grey and black curves respectively. The hexadecapole approximation given by Gould is shown by the green curve. The lower panels shows the relative error of each approximation when compared with the "true" source magnification, calculated using the polygon approximation.

Gould (2008) suggested an error of $0.1 \%$ as an appropriate upper limit for the numerical error in the source magnification calculations. From Figures 4.13 and 4.14 it is shown that multipole-point multipole approximations are more 
Hexadecapole Approximation

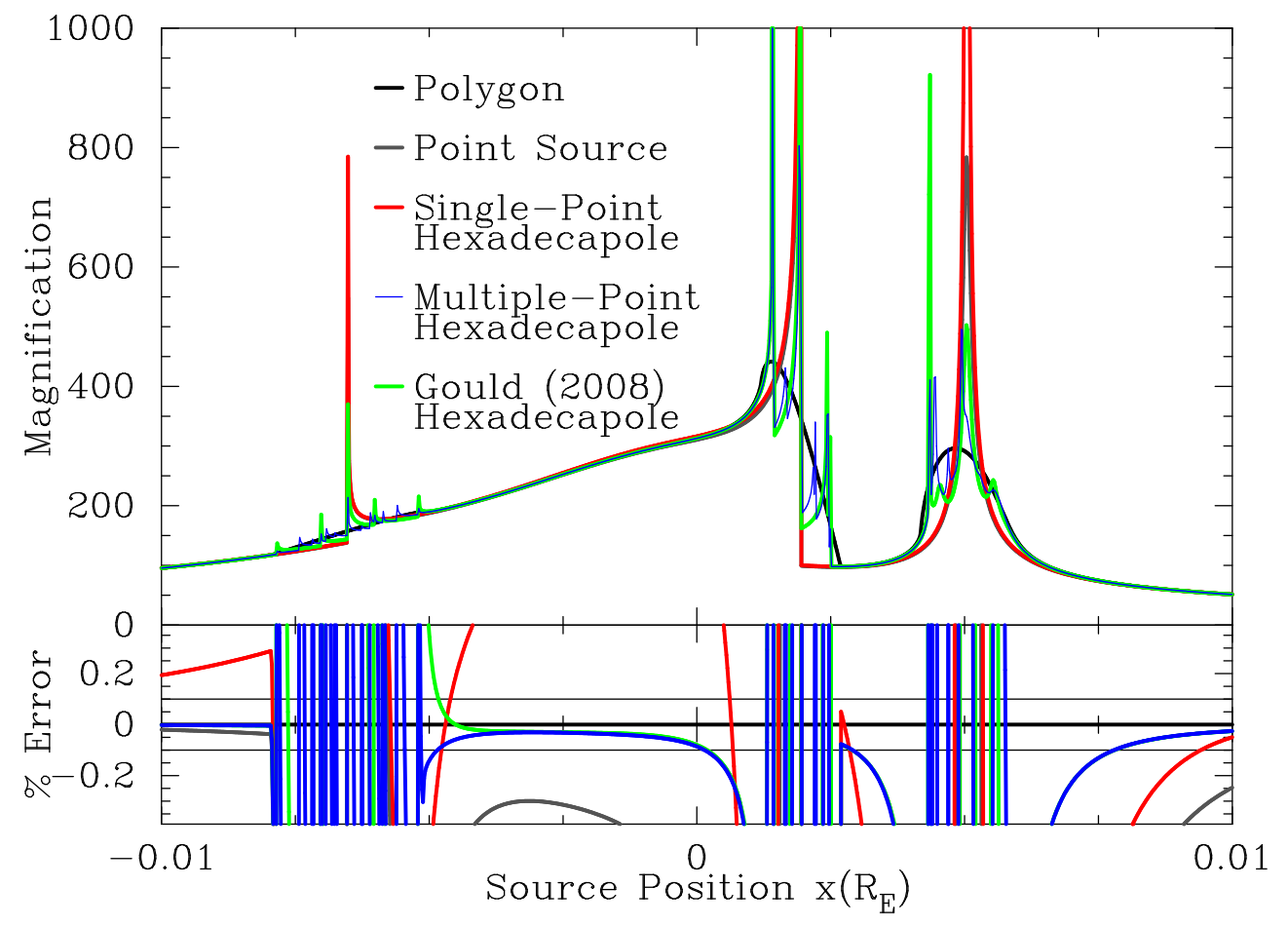

Figure 4.14: The lightcurves plotted using the single-point and multiple-point hexadecapole approximations for a source radius of $0.0005 R_{E}$, for the same event shown in Figure 4.2.

often within the $0.1 \%$ threshold than their single-point equivalents, thus giving a more accurate approximation for the source magnification. It is shown that the hexadecapole approximation given by Gould is no more accurate than the hexadecapole approximation derived earlier in this section.

This figure also shows mini peaks in the multiple-point approximation lightcurves corresponding to the places where each point crosses the caustic curve. These mini peaks are smaller in the hexadecapole method than the quadrapole method. This occurs as the hexadecapole has a larger number of points, so each point source magnification in this approximation is divided by a larger value.

Higher multiple-point multipole approximations are possible, but each higher order approximation requires more points. A quick calculation can show that the next two multipole approximations in the sequence, the 64-pole and the 256pole approximations, which use the Taylor series up to the terms $A_{6} \rho^{6}$ and $A_{8} \rho^{8}$ respectively, requires 25 and 41 points on the source disk respectively. In general, the $2^{2 k}$-pole uses the Taylor series up to the term $A_{2 k} \rho^{2 k}$ and uses $2 k^{2}+2 k+$ 1 points on the source disk. With the number of points rising quadratically 
as each higher order term in the Taylor series is included, it is clear that the number of points required for higher multiple-point multipole approximations soon approaches the number of points required for the polygon approximation. Therefore, it is clearly better to use the polygon approximation than higher multipole approximations, since this explicitly calculates the area of the images and source, giving a more accurate calculation for the source magnification that does not diverge when the source crosses the caustic curves.

\subsection{Selection Algorithm}

When creating a computer program to analyse microlensing data, it is important to make the program both accurate and efficient. Therefore, one needs to balance the speed of the algorithm with its accuracy. An approximation that uses a small number of roots to calculate the source magnification, such as the point source approximation, is fast, but it may calculate the source magnification inaccurately. However, an approximation that uses a large number of point sources, such as the polygon approximation, is more accurate but much slower. Therefore, in order make the program efficient, it is important to determine the appropriate approximation for a particular source position.

Previous work by Chote (2011) suggested that the appropriate approximation could be selected by calculating the curvature (or the gradient of the gradient) of the source magnification at that source position. However, the quadrapole and hexadecapole algorithms give adequate approximations for the source magnification if the higher order terms in the Taylor series are less than $0.1 \%$ or a thousandth of the "true" source magnification at that point (Gould, 2008). Therefore, a more robust method of determining the appropriate approximation would be to calculate the higher order terms in the Taylor series, and compare these terms with the second, fourth and sixth derivatives with the total source magnification.

Calculating the (average) numerical derivatives to check the adequacy of the program requires calculating the point source magnification at a number of points, which in turn increases the amount of computing time. However this 
problem can be overcome using the analytical derivatives. Since each the derivative at a point can be calculated analytically from the point source magnifications, calculating the derivatives this way takes very little extra time. Therefore, although the derivatives at a point are no good in the multipole approximations, they can be used to check if an algorithm gives an adequate estimate of the source magnification.

To determine whether a particular algorithm would give an accurate estimate for the source magnification, the second, fourth and sixth derivatives are calculated at several points on the source disk, and the largest of these are then found (hereafter known as 'maximum derivatives' $A_{2 k \max }$ ). The maximum values for the higher order terms can then be calculated as $A_{2 k m a x} \rho^{2 k}$. If then maximum higher order terms are less than a thousandth of the estimated source magnification, then the algorithm should give an adequate estimation of the source magnification. From experimental investigations, we discovered an additional condition was required, where the higher order terms must be less than $1 \%$ of each individual 'used' term in the Taylor series.

If $A_{2 k a v e} \rho^{2 k}$ represents the terms of the Taylor series calculated using the (average) numerical derivative, then the point source approximations give adequate estimations for the source magnification if,

$$
A_{0}>1000 A_{2 \max } \rho^{2} \quad, \quad A_{0}>1000 A_{4 \max } \rho^{4} \quad \text { and } \quad \mathrm{A}_{0}>1000 \mathrm{~A}_{6 \max } \rho^{6} .
$$

The quadrapole approximation gives an adequate estimation for the source magnification if,

$$
\begin{gathered}
A_{0}+\frac{A_{2 a v e} \rho^{2}}{2}>1000 A_{4 \max } \rho^{4} \quad, \quad A_{0}+\frac{A_{2 a v e} \rho^{2}}{2}>1000 A_{6 \max } \rho^{6}, \\
\frac{A_{2 a v e} \rho^{2}}{2}>100 A_{4 \max } \rho^{4} \quad \text { and } \frac{A_{2 a v e} \rho^{2}}{2}>100 A_{6 \max } \rho^{6} .
\end{gathered}
$$

The hexadecapole approximation gives an adequate estimation for the source magnification if,

$$
\begin{array}{r}
A_{0}+\frac{A_{2 a v e} \rho^{2}}{2}+\frac{A_{4 \max } \rho^{4}}{3}>1000 A_{6 \max } \rho^{6} \quad, \quad \frac{A_{\text {2ave }} \rho^{2}}{2}>100 A_{6 \max } \rho^{6} \\
\text { and } \frac{A_{4 a v e} \rho^{4}}{4}>100 A_{6 \max } \rho^{6} .
\end{array}
$$


The maximum derivative should generally be greater than the (average) numerical derivative $A_{2 k a v e} \rho^{2 k}$. However, this may not always be the case, since the maximum derivative is calculated using a small sample of points, which may or may not be representative across the whole source. If the average derivative is greater than the maximum derivative, this is usually an indication that the point source magnification and its derivatives vary a lot across the source disk, and the polygon approximation should be used to calculate the source magnification.

As a point source approaches a caustic curve, the magnification of the point source increases rapidly and diverges at the caustic curve. After it has crossed the caustic curve it has a different number of images to before it crossed. Therefore, if one point on a finite source disk has a different number of images to another point on the source disk, then the source disk is lying on a caustic curve. When this occurs the polygon approximation (the most accurate approximation) should be used to calculate the source magnification, since the point source, quadrapole and hexadecapole approximations diverge in these circumstances.

Figure 4.15 shows the lightcurve for the same triple lens configuration shown in Figure 4.4 for a source radius of $0.0005 R_{E}$, generated by switching between algorithms. The upper panel shows the grey, red, blue, and black lines indicating the source magnification produced using the point, quadrapole, hexadecapole, and polygon approximations respectively. The lower panel shows the relative error of these approximations from the "true" source magnification, plotted using the polygon approximation. This diagram also shows that the error when modelling this event remains under the $0.1 \%$ threshold. Figure 4.16 shows the critical and caustic curves in this configuration, and shows where in the source track each algorithm was used. 


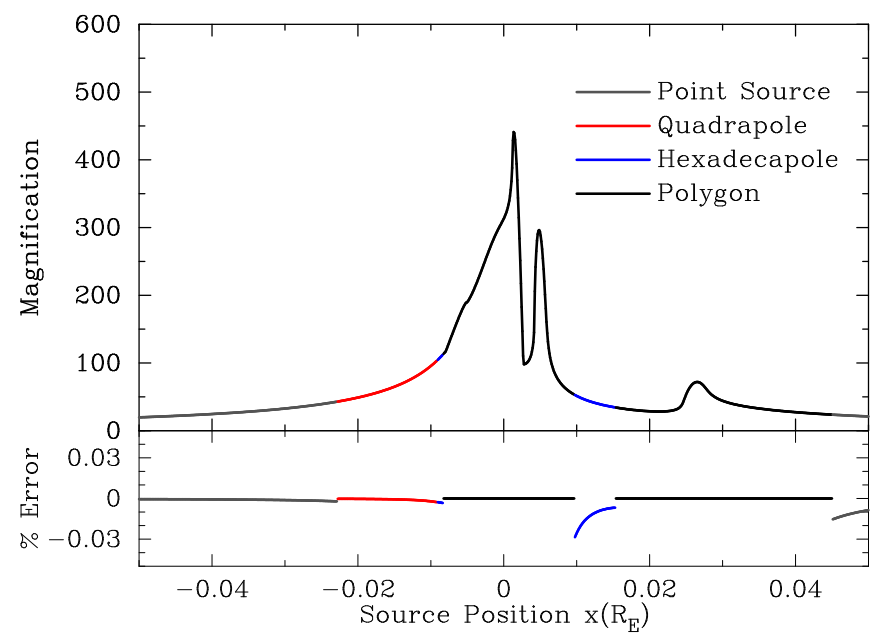

Figure 4.15: The source magnification plotted against source position for the same triple lens event shown in Figure 4.2. This lightcurve was generated using the selection algorithm discussed in Section 4.5, with the point source, quadrapole, hexadecapole and polygon approximations represented by the grey, red, blue and black lines respectively.

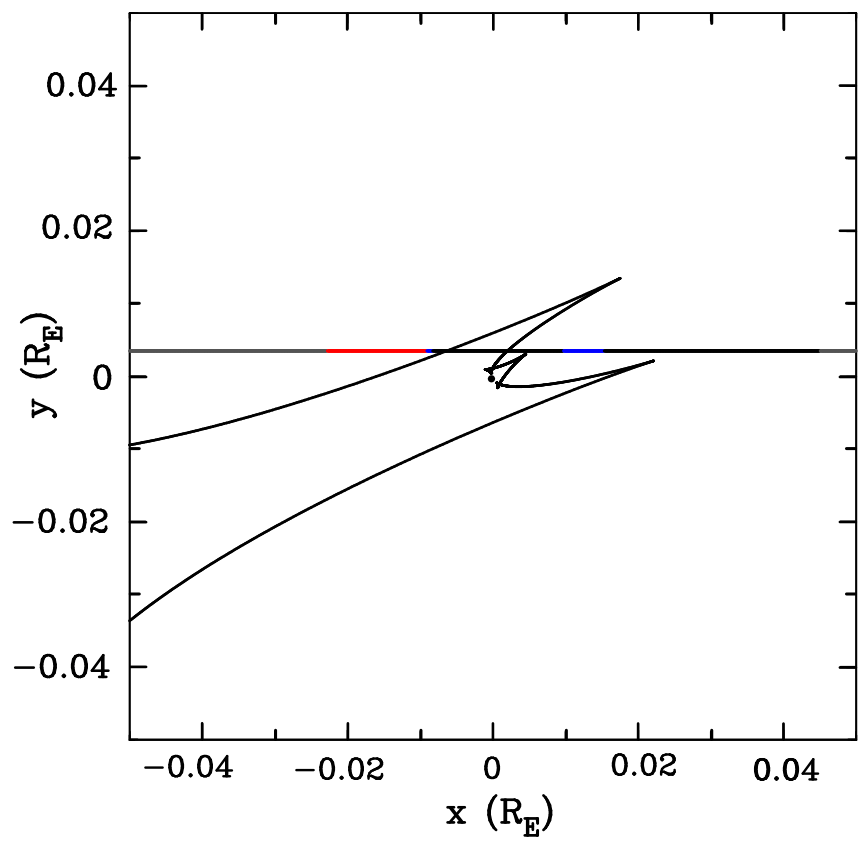

Figure 4.16: The caustic curves and source track for the triple lens event shown in Figure 4.2, demonstrating where each source magnification approximation was used. The grey, red, blue and black lines indicate where the point source, quadrapole, hexadecapole and polygon approximations were used respectively 


\section{Chapter 5}

\section{Numerical Algorithms}

In the previous chapters, we presented an overview of the analytical theory of gravitational microlensing, and discussed the lens equation and its solutions which represent the positions of the images. We then showed how the magnification of the images can be derived from their positions on the image plane. As demonstrated in Chapter 2, the lens equation can be rearranged into the lens polynomial, where the roots of this polynomial are a superset of the image positions. However, the lens polynomial for any multiple lens system has a degree five or greater, which by the Abel-Ruffini theorem, cannot be solved analytically (du Sautoy, 2009). Therefore, these lens polynomials need to be solved using numerical methods.

The purpose of this chapter is to investigate numerical methods, which can be used to solve the lens polynomials. The two algorithms of interest are the Jenkins-Traub algorithm and the Laguerre algorithm, which are discussed in the first two sections of this chapter. Here we give a basic overview of how they work and introduce additional numerical techniques often employed to make these algorithms run more efficiently. The final section examines the accuracy and speed of each algorithm when operating on the lens polynomials, showing how the roots can accumulate numerical errors when using these algorithms. We also investigate the effectiveness of several techniques aimed at minimising these numerical errors, including a method called origin shifting, which has been developed as part of this research. 


\subsection{Laguerre Algorithm}

The Laguerre algorithm is a relatively straightforward polynomial root finding algorithm named in honour of French mathematician Edmond Laguerre. Given a polynomial $P(z)$ with degree $n$ and roots $\zeta_{1}, \zeta_{2}, \cdots \zeta_{n}$, this algorithm finds a sequence of values $z_{0}, z_{1}, \cdots z_{\lambda}, \cdots$ which converges to the root $\zeta_{1}$. The Laguerre algorithm makes the assumption that the root $\zeta_{1}$ is at a small distance $a_{\lambda}$ from $z_{\lambda}$, and all the other roots are at a distance $b_{\lambda}$ from $z_{\lambda}$. The algorithm uses the polynomial derivatives $P^{\prime}(z)$ and $P^{\prime \prime}(z)$ to estimate a value for $a$ and from this value it calculates the next $z$ in the sequence, $z_{\lambda+1}=z_{\lambda}-a$. This process repeats until $a_{\lambda}$ is smaller than the machine precision or $P\left(z_{\lambda}\right)$ is smaller than the acceptable numerical error, implying that the sequence $z_{\lambda}$ has converged to the root $\zeta_{1}$ to within the numerical precision. The algorithm then starts again to find the next root, $\zeta_{2}$. This algorithm is discussed in more detail in Appendix E, Section E.1.

While the assumption that $z_{\lambda}$ is equidistant from the roots $\zeta_{2}, \cdots \zeta_{n}$ is untrue in almost every case, the Laguerre algorithm is almost guaranteed to converge to a root for any complex polynomial.

This algorithm appears in Numerical Recipes as part of the function zroots written in C (Press et al., 1992). zroots is designed so that it starts with the initial value $z_{0}=0$ and finds the roots in roughly increasing order. This code also uses a number of techniques such as deflation, polishing and limit cycle breaking to help the algorithm succeed in finding accurate values for the roots. These terms are explained here.

\subsubsection{Deflation}

Deflation is the process where the calculated root is removed from the original polynomial to give a polynomial with a smaller degree. For example, if the the polynomial $P(z)$ with degree $n$ has the root $\zeta_{i}$, the polynomial $Q(z)=P(z) /\left(z-\zeta_{i}\right)$ has degree $n-1$. The polynomial $Q(z)$ has exactly the same roots as $P(z)$, excluding $\zeta_{i}$, such that $P(z)=\left(z-\zeta_{i}\right) Q(z)$. This process has two advantages; firstly, $Q(z)$ has a smaller degree than $P(z)$, so it generally 
takes less computing time to find the next root in $Q(z)$ than it does for $P(z)$, and secondly, this prevents the algorithm converging more than once to the same (non-multiple) root. An algorithm for deflating polynomials is written in the zroots code (Press et al., 1992), however as this process is done numerically, each numerical root is only known to a finite precision. Therefore, deflation may increase the numerical error in the deflated polynomials and any subsequent roots that are found (Wilkinson, 1965).

\subsubsection{Polishing}

Polishing is the process where, after all the roots are calculated, the Laguerre algorithm is repeated using the newly calculated roots as initial values in the algorithm, but this time the polynomial is not deflated after each root is recalculated. This process aims to reduce the numerical errors in the roots caused by deflation. However, polishing can contribute a significant amount of computing time to the algorithm, as it doubles the number of times the Laguerre algorithm is used. The effectiveness of polishing is discussed in more detail in Section 5.3.

\subsubsection{Breaking Limit Cycles}

A so-called limit cycle is a sequence of $z$ values such that the values do not converge to the root but form an infinite loop. zroots breaks these limit cycles by multiplying the step size $a$ by a multiple of $\frac{1}{8}$ after a set number of steps. If the algorithm has not converged after 10, 20, 30, 40, 50, 60 and 70 steps, the step size $a$ is multiplied by $\frac{1}{2}, \frac{1}{4}, \frac{3}{4}, \frac{1}{8}, \frac{3}{8}, \frac{5}{8}$ and $\frac{7}{8}$ ) respectively. In doing this, the step size is shortened in an attempt to break the limit cycle.

\subsection{Jenkins-Traub Algorithm}

The Jenkins-Traub algorithm is a three stage, convergent, iterative, numerical method developed Jenkins and Traub (1970). A brief overview of the algorithm is presented here, but a more detailed description of the algorithm in given in Appendix E, Section E.2. 
This algorithm works by generating a sequence of polynomials $H^{(\lambda)}(z)$, such that,

$$
\frac{P(z)}{H^{(\lambda)}(z)} \rightarrow z-\zeta_{1} \quad \text { as } \quad \lambda \rightarrow \infty
$$

where $\zeta_{1}$ is the root closest to the origin (i.e. the root with the smallest modulus). The polynomials $H^{(\lambda)}(z)$ are generated using,

$$
\bar{H}^{(\lambda+1)}(z)=\frac{1}{z-s_{\lambda}}\left[\frac{P(s)}{\bar{H}^{(\lambda)}\left(s_{\lambda}\right)} \bar{H}^{(\lambda)}(z)-P(z)\right],
$$

where $\bar{H}^{(\lambda)}(z)$ represents the normalised form of $H^{(\lambda)}(z)$ (i.e. the leading coefficient set to 1$)$.

The first stage of this algorithm, called the "No Shift" stage, generates these polynomials with $s_{\lambda}=0$. The second and third stages of this algorithm use $s_{\lambda} \neq 0$, which effectively aims to shift the origin closer to the root so that $\zeta_{1}$ is smaller, and $\bar{H}^{(\lambda)}(z)$ converges faster to $P_{i}(z)$. Although stage one is not necessary from a theoretical perspective, it is useful for accentuating the roots. Stage one is terminated and stage two begins after 5 iterations (i.e. $M=5$ ), as determined by numerical experience (Jenkins and Traub, 1970).

The second stage, called the "Fixed Shift" stage, uses a fixed value for $s_{\lambda}=s$ to shift the origin closer to the root. The fixed shift $s$ is defined as the real positive value $\beta$ multiplied by a random phase (or $|s|=\beta$ ) where $\beta$ is an estimate of the lower bound for the modulus of the roots of $P(z)$. The method used to obtain the value for $\beta$ is discussed in Appendix E, Section E.2. Stage two is terminated and stage three starts, when so called weak convergence is satisfied in two successive iterations. Weak convergence is said to occur when the following criterion is met,

$$
\left|\frac{P(z)}{\bar{H}^{(\lambda)}(z)}-\frac{P(z)}{\bar{H}^{(\lambda-1)}(z)}\right| \leq \frac{1}{2}\left|s-\frac{P(z)}{\bar{H}^{(\lambda-1)}(z)}\right| .
$$

If weak convergence is not satisfied after a set number of iterations, $\beta$ is given a new random argument to create a new fixed shift $s$, and stage two starts again.

The third stage, called the "Variable Shift" stage calculates a new value for $s_{\lambda}$ based on the polynomial $H^{(\lambda)}(z)$. This stage uses a process identical to Newton's method to calculate the root at each iteration. When $P(z)$ is smaller than the 
acceptable numerical error, stage three is terminated, the polynomial is deflated, and the algorithm starts finding the next root, $\zeta_{2}$.

This algorithm, as it is appears in the FORTRAN subroutine CPOLY (Jenkins and Traub, 1972), also uses deflation and breaking limit cycles to help it succeed in finding accurate values for the roots. This FORTRAN code was converted into $\mathrm{C}$ for the calculations and plots used in this chapter. In a similar style to the Laguerre algorithm, the roots of a polynomial are found in roughly increasing order to reduce the numerical errors caused by deflating the polynomial by a large root. Like the Laguerre algorithm, the Jenkins-Traub algorithm is also almost guaranteed to converge to a root for any complex polynomial. However, in contrast to the Laguerre algorithm, which has limited theoretical understanding, the Jenkins-Traub algorithm has a solid theoretical base.

\subsection{Algorithm Accuracy and Efficiency}

When selecting an algorithm to analyse microlensing data, it is important to consider both the speed and accuracy of the algorithm. Mekwi (2001) showed that zroots (Laguerre algorithm) is faster than CPOLY (Jenkins-Traub algorithm) for polynomials up to degree 90, when both algorithms are written in FORTRAN, but implied that CPOLY finds the roots more accurately. In this section, we discuss the speed and accuracy of these two algorithms when written in $\mathrm{C}$, focusing on how these algorithms can be used to analyse microlensing data.

As discussed in the previous chapters, the distinct images produced in a microlensing event cannot be resolved, so microlensing events are observed by measured the changing luminous flux (or source magnification) over time giving what is known as a lightcurve. The analysis of a microlensing event requires producing up to millions of theoretical microlensing lightcurves to find the theoretical lightcurve that fits best with the observed lightcurve. Each theoretical lightcurve may require finding the root positions for up to 1000 source positions along a line (hereafter known as a source track) so the root finding algorithm needs to be fast and efficient.

However, when generating theoretical lightcurves, it is also important to dis- 
tinguish the physical images from unphysical roots. Only the physical images contribute to the total magnification of the source, so the misidentification of some images as unphysical when they should be physical, or vice versa would lead to an incorrect value for the source magnification. As mentioned in Section 2.6, the number of physical images is $N+1+2 k$ where $k$ is a non-negative integer. If this does not match the number of physical images found using the root-finding algorithm, then it is likely that the calculated source magnification is incorrect. Therefore, as well as being fast, the root finding algorithms must also be accurate so that each lightcurve is calculated accurately.

As mentioned in Chapter 3, a microlensing event with $N$ lenses will always have a total parity of $1-N$. If the total parity is not calculated as $1-N$, it is an indication that some of the roots have been misidentified as being unphysical when they are in fact physical, or vice versa, so the calculated source magnification is likely to be incorrect.

\subsubsection{Accuracy and Efficiency of Numerical Techniques}

A main aspect of this research was to investigate numerical techniques to improve the accuracy and efficiency of the root-finding algorithms. The speed and accuracy of these techniques were tested using 12 specifically developed programs written in C, the largest of which had a total of about 2000 lines C code. Each program calculated the image positions for 1000 lightcurves each with a randomly generated lens system with 2, 3 and 4 lenses, and each consisting of 1000 source positions.

Wambsganss (1997) proposed that planets should be detectable with current telescope technology with mass ratios in the range $10^{-3}-10^{-5}$ in the so-called 'lensing zone', in the range $0.6 R_{E}-1.6 R_{E}$ from the primary lens. The lensing zone represents the region where a planetary configuration will give planetary caustics inside the Einstein ring, up to $1.0 R_{E}$ from the primary lens. Therefore, each lens configuration has planets randomly positioned inside the lensing zone.

Gaudi et al. (1998) showed that the probability of detecting planets of the same mass as Jupiter in the lensing zone is nearly $100 \%$ when the impact pa- 
rameter (closest distance between the source and the origin) is no greater than $0.1 R_{E}$. Therefore, each configuration includes a random impact parameter in the range $10^{-1}-10^{-4}$ with a source track parallel to the $\mathrm{x}$-axis from $x=-1.0 R_{E}$ to $x=1.0 R_{E}$, composed of 1000 source positions.

If the algorithm failed to return the correct number of images $(N+1+2 k)$ or the correct parity $(1-N)$ for at least one source position in the source track, then the whole lightcurve was labelled as a "failed lightcurve". The "successful lightcurves" were the lightcurves that did not fail. Note that a failed lightcurve would still find $N^{2}+1$ roots using the root-finding algorithm for each source position, but did not find $N+1+2 k$ (physical) images for every source position. A failed lightcurve is an indication that roots of the lens polynomial were calculated inaccurately by the algorithm.

\section{Polishing}

As mentioned previously in Section 5.1, polishing is the process where the roots are put through the root finding algorithm again with the undeflated polynomial.

CPOLY (Jenkins-Traub) by itself does not contain a polishing method, but the Laguerre polishing method used in zroots can be easily implemented in CPOLY. While polishing aims to increase the accuracy of the roots, it may also increase the computing time.

We tested the speed and accuracy of these two root-finding algorithms by implementing zroots and CPOLY into the 12 specifically developed programs and compared the results. We found that the Laguerre algorithm is significantly faster at solving the polynomials than the Jenkins-Traub algorithm, and polishing increases the amount of computing time for both Laguerre and Jenkins-Traub. It was also found that polishing increasing the time taken by more than double in the quadruple lens case. However, we determined that polishing did not significantly improve the accuracy roots. All algorithms in the quadrapole cases, with and without polishing, failed to find all the physical images in all the lightcurves (i.e. the algorithms had a success rate of $0 \%$ in the quadrapole case). 


\section{Precision}

At its core, the primary cause of numerical error is the compounded effect of two types of errors. The first type, truncated error, is where mathematical equations are simplified to make them usable for calculations. The second type of error, precision error, is where each value is only stored on a finite number of bits, so each number only has a finite precision. Values expressed using single-floating precision-point are truncated to the nearest 23bits (approximately 7 digits), while values expressed using double-floating precision-point are given to the nearest 52 significant bits (approximately 16 digits). Also, using a higher precision to express these values would reduce the precision error and may decrease the numerical error in each root.

The need for higher precision for a triple lens microlensing event has been noted by Bennett (2010), who suggested using quadruple-floating precision-point (128 bits or 34 digits) to avoid the errors caused by precision. However, he mentioned that quadruple-precision can be up to 100 times slower than doubleprecision in some compiler implementations.

We found that quadruple precision increased the amount of time to calculate the roots by a significantly large factor, up to 90 times longer in some cases, but it did not significantly increase the accuracy of the roots. Therefore, we reasoned that it is not economic to use quadruple-precision in the modelling of gravitational microlensing events.

Interestingly, we discovered that image positions calculated using singleprecision floating-point took about the same time to find the roots as doubleprecision in most cases. This can be explained by noting that these simulations were run on a laptop with an x86 Intel Pentium microprocessor. This type of processor implements double-precision floating-point, so single-floating point values are emulated by extending them. This conversion from single-floating point to double-floating point values adds time to the calculations. On others computers with different microprocessors, single-floating point precision calculations may be faster, however we discovered that the roots calculated using single-precision floating-point were significantly more inaccurate than roots found using double- 
and quadruple-precision. Thus, single-precision is too inaccurate to use when analysing microlensing data.

\section{Initial Guess}

To simulate a lightcurve, a computer program calculates the roots for each source position in a series of source positions along a line. A lightcurve with 1000 source positions may have adjacent source positions less than $0.001 R_{E}$ apart. The lens equation is continuous everywhere except at the lens positions, and a small change in source position usually gives a small change in the root positions. Therefore, the roots for a particular point source are generally expected to be close to the roots from the previous source position. For this reason, the roots from the previous source position could be used as the initial values for the root finding algorithm to find the roots for a particular source position.

The 'default' initial value for the zroots subroutine is zero. The 'default' initial value for the CPOLY subroutine is the lower bound of the modulus of the roots, with a random argument. These default initial values were used to obtain the plots presented earlier in the chapter. As part of this research, both these algorithms were adapted so the roots from the previous source position were used as the initial values in the algorithms. By doing this, the algorithm was expected to converge more quickly to the roots, so each theoretical lightcurve would be generated faster. In this strategy, the 'default' initial values were only used for the first source position in the lightcurve, and the previous roots were used as initial values for every subsequent source position.

We found that Jenkins-Traub without polishing, using the previous roots as initial values simulates the lightcurve in about $41 \%$ of the time it takes using the default initial values. Using Laguerre without polishing with the previous roots simulates the lightcurve in $64 \%$ of the time it takes using the default initial values. However, we found that there is no significant difference in the accuracy of the image positions when using the default initial values or using the previous roots as the initial values. 


\subsubsection{Origin Shifting}

While investigating the accuracy and efficiency of these numerical techniques, we discovered that only about 2 physical images were identified in the triple and quadruple lens cases whenever the source was far from the primary lens, compared to the $N+1$ physical images that are expected in these cases. In some triple lens systems, the algorithm returned 2 physical images when the source no less than $0.5 R_{E}$ from the primary lens, and in some quadruple lens systems, this occurred when the source was no less than $0.05 R_{E}$ from the primary lens.

As explained in Section 2.6, a source far from the primary lens corresponds to roots that are close to the source position or lens positions, with $N-1$ unphysical roots clustered around each lens, and one physical image near the source and each lens. From our numerical investigations, we found that the root close to the source was always correctly identified as a physical image, and of the $N$ roots clustered around the primary lens, one was identified as physical and $N-1$ as unphysical, implying that all the roots around the source and primary lens were correctly identified as physical or unphysical. However, in these numerical investigations, all the roots close to the planetary lenses were often returned as unphysical. This indicates that the roots around planetary lenses were inaccurate, since one root close to each lens should always be identified as a physical image.

This misidentification is caused in part by $\epsilon /(z-r)$ term in the lens equation. i.e. when the root, $z$, is close to a lens, $r$, a small inaccuracy on the image plane corresponds to a large inaccuracy on the source plane when the root is inverse ray traced. However, the fundamental cause of the misidentification is the inaccuracies in the roots. The main reason the roots around the primary lens are not misidentified is because these roots we not inaccurate when they were found via the polynomial root finding algorithm.

In the previous sections of this chapter, the lens polynomial has been constructed and the roots have been found with the origin of the complex plane positioned at the primary lens, so that all planetary lenses and source positions are given in terms of their distance from the primary lens. However, it was discovered that when the origin was shifted to a particular planetary lens, the roots 
surrounding that lens becomes much more accurate, with one physical image and $N+1$ unphysical roots correctly identified around this root. However, when this origin shift is applied, the roots clustered around every other planetary lens become more inaccurate.

Therefore, to reduce the inaccuracies in all the roots, we have to shift the origin from the original origin to each of the lenses, and calculate and record the position of these roots for each origin shift. For a configuration with $N$ lenses, this can be achieved by creating $N$ copies of the source track and the lens positions, each where the origin has been shifted to a different lens. Using each of these origin-shifted source tracks and lens positions, $N$ polynomials are created, each with $N^{2}+1$ roots, using the method described in Chapter 2. Each of these polynomials will have the origin at a different lens. These polynomials are then solved, and the roots are shifted back to the original origin.

After polynomials are solved and shifted back to the common origin, there are $N$ representations for each of the $N^{2}+1$ roots. From these, the most accurate representation for each root needs to be selected. The Jenkins-Traub and Laguerre algorithms find the roots in roughly increasing order, so when the roots are shifted back to the original origin, the roots are ordered roughly from closest to further from its corresponding lens origin. Therefore, before this selection process occurs, the roots needed to be sorted, so that each root from each origin matches up with their respective roots from the other origins.

Sorting Algorithm If the roots are calculated in a computer program, these roots can be stored in arrays. The roots can then be shifted using the following procedure:

1. Take the first root from the array with the primary lens at the origin. Call this root $z_{1,1}$.

2. Iterate through the roots with the secondary lens at the origin, and find the root closest to $z_{1,1}$. Call this root $z_{2, i}$

3. Swap $z_{2, i}$ with $z_{2,1}$, the first root in the array with the secondary lens at 
the origin. Now the first root in the primary lens array and the first root in the secondary lens array correspond to the same root from two different origins.

4. Repeat steps 2 and 3 with the remaining arrays. When this is complete, the first root in all the arrays correspond to the same root from different origins.

5. Repeat steps $1-4$ with the second root from the array with the primary lens at the origin $\left(z_{1,2}\right)$ and collect the corresponding roots into a group using the remaining (unsorted) roots in the other arrays.

6. Repeat step 5 with the rest of the roots. When this is complete each root in each array will be matched with its corresponding root in the other arrays.

Once the roots from each polynomial have been sorted to match their corresponding roots from the other origins, we need to select the most accurate value for each root. As we discovered earlier, the roots were most accurate when they were closest to the origin. Therefore, to find the most accurate values, we must find the closest lens to each root. The appropriate value for the root is then taken from the origin associated with that lens.

We tested origin shifting technique conjunction with the polishing technique and the different initial values to find the ideal algorithm for finding the root positions. We found that origin shifting increases the computation time of the algorithm by a factor of about 1.7 in the binary lens case, a factor of about 3.0 in the triple lens case, and a factor of about 2.8 in the quadruple lens case. However, it significantly reduces the proportion of lightcurves that fail. Without origin shifting, all algorithms had a $0 \%$ success rate for the four lens systems, but with origin shifting, all the algorithms had a non-zero success rate, demonstrating that origin shifting generally calculates the roots more accurately. The only algorithm to give a $100 \%$ success rate in the four lens case was the Jenkins-Traub algorithm with origin shifting and default initial values but without polishing. 


\section{Chapter 6}

\section{Modelling Gravitational Microlensing Events}

As mentioned in the previous chapters, one of the main reasons for studying gravitational microlensing, and indeed, the primary reason at VUW (Victoria University of Wellington) is to search for extrasolar planets. But, as there is no analytical relationship between the lightcurve and the physical properties of the lens system (such as mass, orbital distance, etc.), there is no easy method of extracting values of these parameters from an observed lightcurve. However, the parameters for a microlensing event can be obtained by comparing the observed lightcurve with lightcurves from theoretical microlensing events. Each theoretical event can be described by a set of parameters, which specifies the source track, the finite size of the source, the positions and the relative masses of the each lens, as well as any orbital motion, such as parallax, xallarap and lens motion.

Once a model lightcurve has been produced for a particular event, the model lightcurve is compared against the observed data. The 'quality of the fit' of a theoretical lightcurve with the observed lightcurve can be quantitatively described by the $\chi^{2}$ value, given as,

$$
\chi=\sum_{i}^{n} \frac{A\left(t_{i}\right)-O_{i}}{\sigma_{i}},
$$

where $O_{i}$ and $\sigma_{i}$ are the observed amplification and its uncertainty respectively at the $i$ th data point out of a total of $n$ data points. $A\left(t_{i}\right)$ is the calculated amplification for the theoretical lightcurve at time $t_{i}$, corresponding to the $i$ th data point. Assuming the uncertainties are estimated correctly, each term in the 
sum should be on the order of 1 if the theoretical data agrees with the observed lightcurve. Therefore, if $m$ is the number of parameters, it can be shown that a "good model" should have $\chi^{2} /(n-m) \sim 1$, where $n-m$ gives the degrees of freedom, where smaller $\chi^{2}$ values indicate a better fit.

Therefore, the analysis of a particular microlensing lightcurve requires searching the $m$-dimensional parameter space to find the theoretical lightcurve with the smallest $\chi^{2}$. Two methods can be implemented to find the smallest $\chi^{2}$, the first by iteratively converging to these points, and the other by stochastic methods, such as Markov chain Monte Carlo algorithms. These searches require the simulation of a large number of theoretical microlensing events to find the best-fitting theoretical lightcurve. Therefore, massive computing power is required for these parameter searches and the lightcurves need to be produced as quickly as possible without losing accuracy in the calculations. More details on comparing and fitting models can be found in Korpela (2007).

\subsection{Modelling Procedure}

At VUW, the optical astrophysics team lead by Prof Denis Sullivan is currently developing a software package that will ultimately be used to analyse microlensing events. This software package originated as an inverse ray tracing based package called MLENS developed by Korpela (2007). However, this was later replaced by the software package mlens2 developed by Chote (2011), which used semianalytical method to calculate a $\chi^{2}$ value and generate lightcurves. The research presented in this thesis builds upon the mlens2 code to make it more robust and efficient.

In its current form, this package contains total of approximately 13000 lines of $\mathrm{C}++$ code, and is composed of two executables: MLjob and MLserver. The MLjob executable contains the logic required to generate a lightcurve given a set of parameters, using the numerical methods discussed in this thesis. This executable is used to calculate the $\chi^{2}$ value for the model lightcurve, for a set of observational data. The MLserver executable controls the searches in the parameter space, given some initial conditions, to find the best fitting model for 
an observed lightcurve. As of mid-2012, the algorithms required to efficiently search the parameter space have not yet been fully implemented in the MLserver code, so large parameter searches are not possible. However, it is expected that a Markov chain Monte Carlo algorithm will be implemented in the code in the near future, which will make large parameter searches possible. For more information on these executables, see Chote (2011).

The mlens2 package receives the parameters in the form of a plain text control files. The parameters in each control file define the lens positions and masses, the source size, the source track, and any orbital motion such as parallax, xallarap or lens motion. Given these parameters, a collection of source targets are positioned along the source track on the source plane. For each source position, the lens polynomial is constructed using the semi-analytic method, and is then solved using a root-finding algorithm. Once the roots of the polynomials are found, the roots that corresponded to (physical) images are identified and the amplifications of these images are then summed to give the amplification of the source. The amplification can be calculated using the point source approximation, the multipole approximations, or the polygon approximation

The current version of the mlens2 code differs in a number of ways from the code developed by Chote in 2010. Firstly, the lens polynomials and critical curve polynomials in the current version of mlens2 are constructed differently from the 2010 version of the code. The current version of the code uses the lens polynomials and critical curve polynomials presented in this thesis (Chapters 2 and 3 and Appendices B and C), while the 2010 version of the code uses the polynomial coefficients given by Chote (2011).

Secondly, the current version of the code incorporates the quadrapole and hexadecapole approximations discusses in Chapter 4, whereas the 2010 version did not include the quadrapole approximation, and used the hexadecapole approximation from Gould (2008). The quadrapole approximation is faster than the hexadecapole approximation but more accurate than the point source approximation. Thirdly, the current version of the code uses the selection algorithm described in Section 4.4, whereas the 2010 version of the code used the selection 
algorithm from Chote (2011). We found that the current version of the code used the quadrapole approximation in some places where the 2010 version of the code uses the hexadecapole approximation. The use of the quadrapole approximation has been a factor in making the current code faster, as demonstrated in Sections 6.2 and 6.3 .

Fourthly, the origin shifting technique was been implemented in the current version of the code, but was not present in the 2010 version of the code. In Chapter 5 , we showed that Jenkins-Traub with origin shifting, the default initial value and without polishing was the only algorithm to give a 100\% success rate for all lens configurations up to and including quadruple lens systems. However, origin shifting was found to be reasonably time-consuming, taking approximately 1.7, 3.0, and 3.8 times longer than without origin-shifting for a lens system with 2,3 , and 4 lenses respectively. To ensure time is not wasted using this computationally expensive technique when it is not required, origin shifting was implemented in such a way that mlens2 initially attempts to find the roots using the algorithm without origin shifting, but falls back on the more time consuming but more accurate origin shifting algorithm if the algorithm fails to return the expected number of images $(N+1+2 k$ where $k=0,1,2, \cdots)$ or total parity $(1-N)$.

Lastly, the current version uses this algorithm without polishing, while the 2010 version of the code used the Jenkins-Traub algorithm with Laguerre polishing. The absence of the polishing technique has been another factor in making the current code faster, as demonstrated in Sections 6.2 and 6.3. Both of the current and 2010 versions of the code used the default initial values for the JenkinsTraub algorithm. In Chapter 5, we mentioned that using the previous roots as the initial values for the algorithm was faster than using the default initial values. However, these results were obtained using a set of smaller $\mathrm{C}$ programs that did not have the added complexities contained within the mlens2 package. Due to the complex structure of mlens2, this package does not always calculate the amplifications of the source positions sequentially from one end of the source track to the other. When this package uses observational data the algorithm often jumps relatively large distances between the source positions and the size of 
these jumps depends on time of data points. Thus, using previous roots as initial values becomes inefficient when finding the root positions in mlens2. Therefore, using the previous roots as the initial values for the algorithm was found to be more appropriate for the mlens2 code.

We here implement the MLjob executable in the current version of the mlens2 software to model the gravitational microlensing events OGLE-2006-BLG-109 and MOA-2009-BLG-319 and discuss the result in the following sections.

\subsection{MOA-2009-BLG-319}

MOA-2009-BLG-319 was the tenth planetary microlensing event to be published, making MOA-2009-BLG-319Lb the eleventh extrasolar to be discovered via gravitational microlensing (Miyake et al., 2011). This event was first detected by the MOA collaboration on 20 June 2009. This data corresponds roughly with

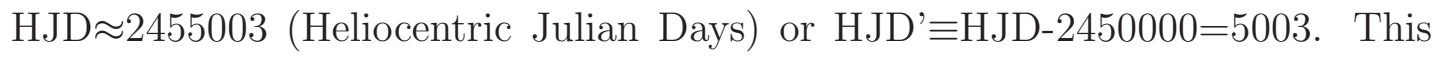
event was initially announced as a normal microlensing event; however a preliminary model indicated that it was a high-magnification event, so at once MOA began follow-up observations with the Mt. John Observatory. Over the next two nights, the $\mu \mathrm{FUN}$, RoboNet, and MiNDSTEp collaborations also began to observe this event. The first (weak) caustic crossing occurred three days after the initial discovery but was initially described as "low-level systematics". It was not until the second caustic crossing, 14 hours later, that the $\mu \mathrm{FUN}$ collaboration sent out an anomaly alert, indicating the discovery of a planetary lens.

The host star for this event is a K- or M-dwarf star type and is located in the inner Galactic disk or Galactic bulge, at a distance of $D_{L}=6.1_{=1.2}^{+1.1} \mathrm{kpc}$ from Earth. The mass of the host star, $M_{L}=0.38_{-0.18}^{+0.34} M_{\odot}$, was obtained using Bayesian analysis, based on the measurements for the Einstein crossing time, $t_{E}$, and the angular Einstein radius, $\theta_{E}$, using a standard Galactic model.

The best fitting model (without parallax) for this event gave a planetary mass ratio of $q=(3.95 \pm 0.02) \times 10^{-4}$ and a lens separation of $d=0.97537 \pm 0.00007 R_{E}$. Using Bayesian analysis, this corresponded to a planet with a mass of $M_{p}=$ $50_{-24}^{+44} M_{\oplus}$ or half the mass of Saturn, at a distance $a=2.4_{-0.6}^{+1.2}$ AU from its host 
star.

These model parameters for the mass ratio and lens separation were taken from Miyake et al. (2011) and converted to the coordinates system used by mlens2. The lightcurve for this model is presented in Figure 6.1, showing this model agrees well with the observational data when using the mlens2 software. The control file for this model is shown in Appendix F, Figure F.1.

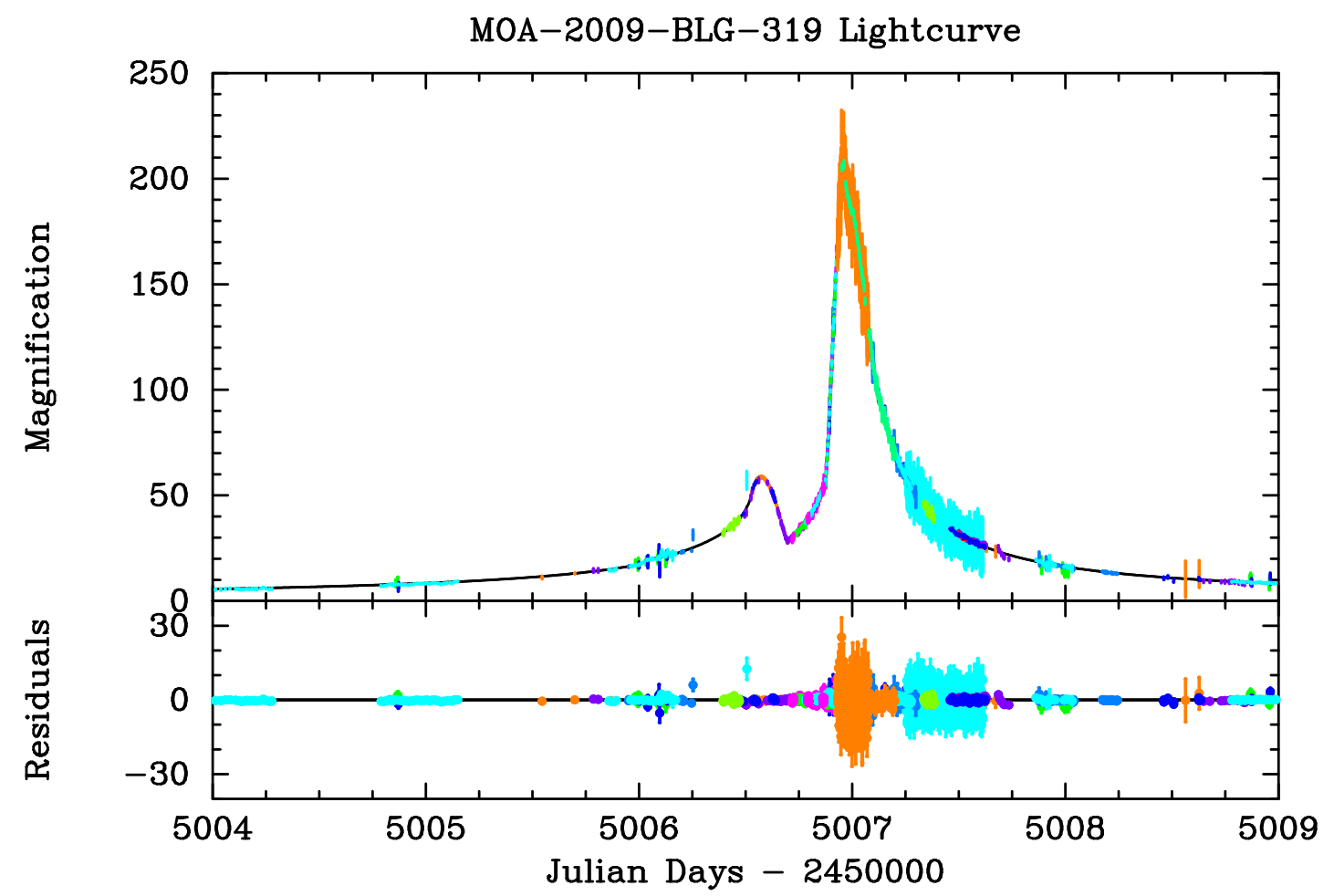

Figure 6.1: Model lightcurve for MOA-2009-BLG-319, generated using the current version of mlens2.

This model indicates that the source crossed the caustic curves at four distinct times. The first crossing observed by MOA occurred at HJD' $=5006.05$ and was described as weak, producing little effect on the lightcurve. This was followed by the second caustic crossing at HJD' $=5006.6$. The third caustic was described as a strong caustic entry and produced the peak in the lightcurve with $A_{\max } \sim 205$ at HJD' $=5006.96$. The fourth and final caustic crossing occurred quickly after at HJD' $^{\prime}=5007.0$ with a source magnification of $A \sim 180$. This final caustic crossing was well observed by 16 telescopes, giving continuous photometric monitoring, with gaps no larger than 5 minutes. The magnification map for this model is presented in Figure 6.2, showing the caustic curves and the source track. 


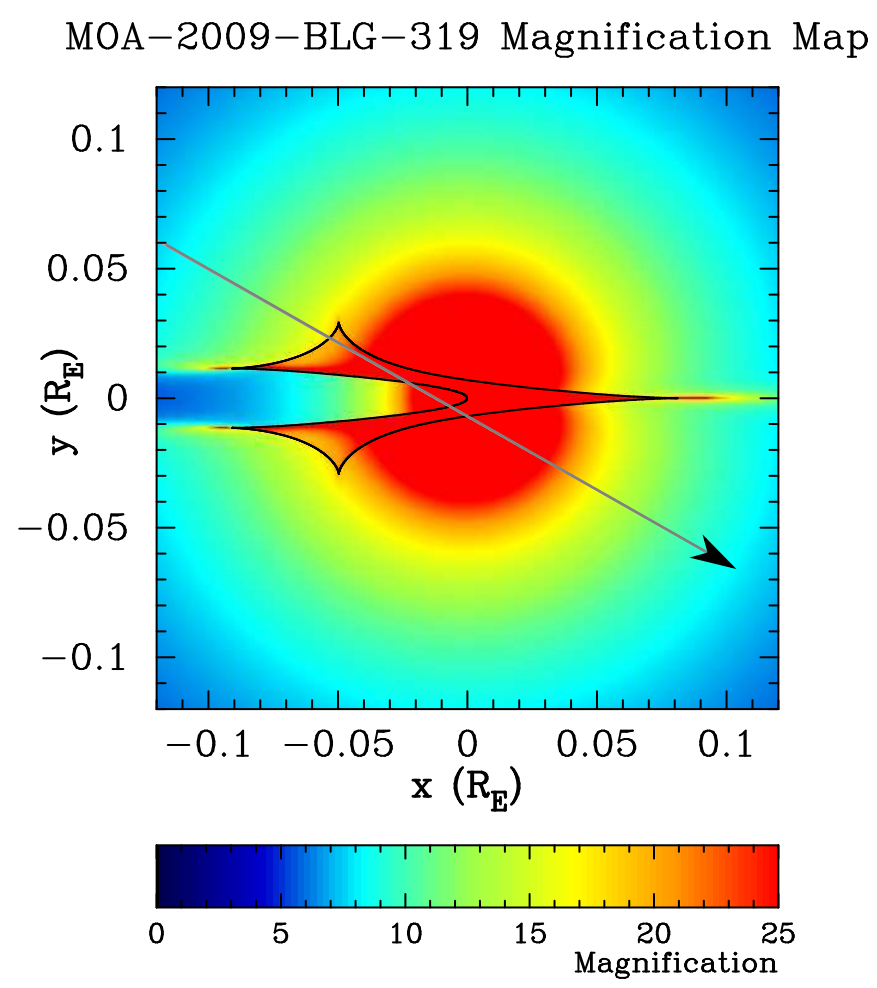

Figure 6.2: Magnification map for MOA-2009-BLG-319, generated using the current version of mlens2, showing the caustic curves in black. The line of the source track is in grey, with the direction of the track shown by the arrow head.

When the mlens2 was used to calculate a $\chi^{2}$ value it returned a value of $\chi^{2}=7746.86$ in 176 seconds. This used 18 observational data sets, containing a total of 2801 data points. In the paper published by Miyake, the model has $\chi^{2}=7023.8$, however this value was calculated using a total of 7210 data points, so it cannot be compared directly to the $\chi^{2}$ value obtained using mlens2.

However, this $\chi^{2}$ value and the time taken to generate this model can be compared to the late 2010 version of mlens2. This version of mlens2 does not contain many of the improvements to the code presented in this thesis. Therefore, comparing the $\chi^{2}$ values and the total run times of the current with this earlier version of the code is a way of quantifying the improvements in the accuracy and efficiency of the numerical methods presented in this thesis. When the control file and data sets from in Figure 6.4 were run on this earlier version of mlens2, a $\chi^{2}$ value of 7746.95 was calculated in 230 seconds. These results show that the current version of mlens2 is also about $23 \%$ faster than the the earlier version of the code. 


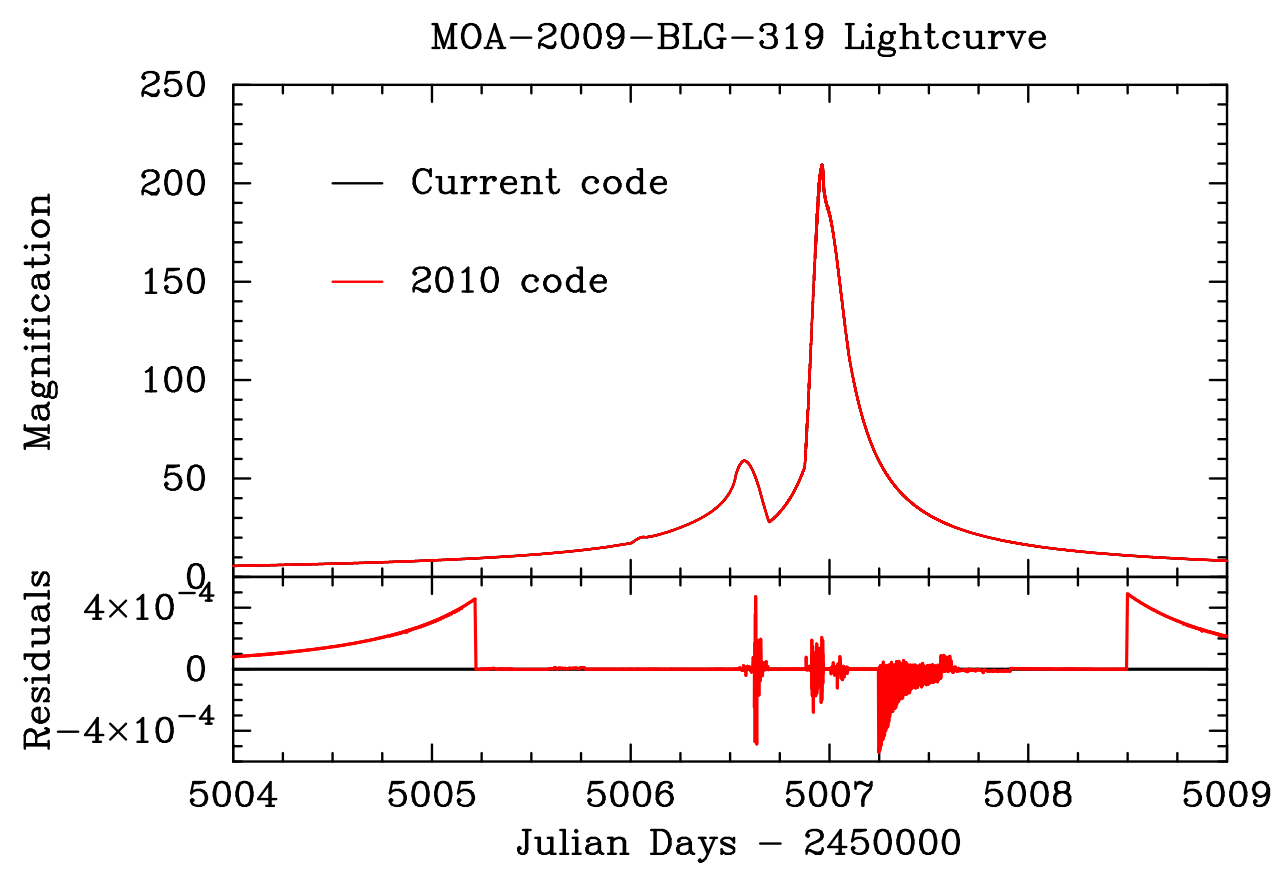

Figure 6.3: Comparison of the model lightcurves for MOA-2009-BLG-319, generated using the current version (black) and the 2010 version (red) of mlens2. The lower panel shows the residual between the 2010 version of the code, and the current version, demonstrating that the two codes do not differ by more than $0.0006 \%$.

Figure 6.3 shows a comparison between the current and the 2010 version of the code. The black line shows the lightcurve generated by the current code, while the red line shows the lightcurve generated by the 2010 version of the code. This plot shows that the difference between the calculated amplification between the two versions of the code remains under $0.0006 \%$ for any source position. Since $0.1 \%$ is the upper limit for the numerical error in amplification calculation, as suggested by Gould (2008), this result implies that improvements to the code has not had a significant effect on accuracy of the code for this particular model.

Although the current version of the mlens2 calculates the $\chi^{2}$ value faster than the 2010 version, it is still an order of magnitude slower what is required to economically search for the best fitting model in the parameter space. When limb darkening was removed from the code, the calculation time dropped to 67 seconds with a $\chi^{2}$ value of 9318.15 , and when the polygon approximation was completely removed from the code, the calculation time dropped to less than a second but with a $\chi^{2}$ value of over 9 million! This demonstrates that the polygon approximation, while reasonably accurate, is very time consuming due to the 
large number of source points used to make this calculation, especially when limb darkening is included in the model.

\subsection{OGLE-2006-BLG-109}

OGLE-2006-BLG-109 was the fifth planetary lens microlensing event to be published, and the first event to be modelled with more than one planetary lens (Gaudi et al., 2008). This event announced as a potential planetary event by the OGLE collaboration on 28 March 2006, or HJD' $\approx 3822$. At once, the $\mu \mathrm{FUN}$ and RoboNet collaborations began follow-up observations, as the source magnification of the event increased. The first caustic crossing occurred seven days later on 5 April, and from this deviation, a preliminary model predicted a caustic crossing on 8 April. This deviation did in fact appear on 8 April, however an additional deviation was observed about 12 hours after the first deviation, indicating the presence of a second planet.

The model parameters for the best-fitting model were taken from Bennett et al. (2010). To analyse this event using mlens2, the parameters for the event were taken from this paper and converted to the coordinates system used by mlens2. The parameters in this control file included parameters for controlling the effects of parallax, xallarap and lens motion. The control file containing these parameters is presented in Appendix F, Figure F.2.

Figure 6.4 presents the lightcurve for this published model, generated using the current version of mlens2. This plot shows that this model appears to agree well with the observational data. The mlens2 calculated a $\chi^{2}$ value of 2511.33 in 94 seconds using 1193 observational data points for this model. Due to the orbital motion of the lens, the caustic curves for this event transformed over time. This is demonstrated Figure 6.5, where the red, magenta, and blue critical curves correspond to HJD'=3820,3830,3840 respectively

In the paper published by Bennett et al. (2010), the model has a $\chi^{2}=2542.06$. However we cannot directly compare this $\chi^{2}$ value with the value calculated using mlens2, since the $\chi^{2}$ value calculated by mlens2 did not use the final set of data used by Gaudi et al. (2008) or Bennett et al. (2010). The data sets used by 


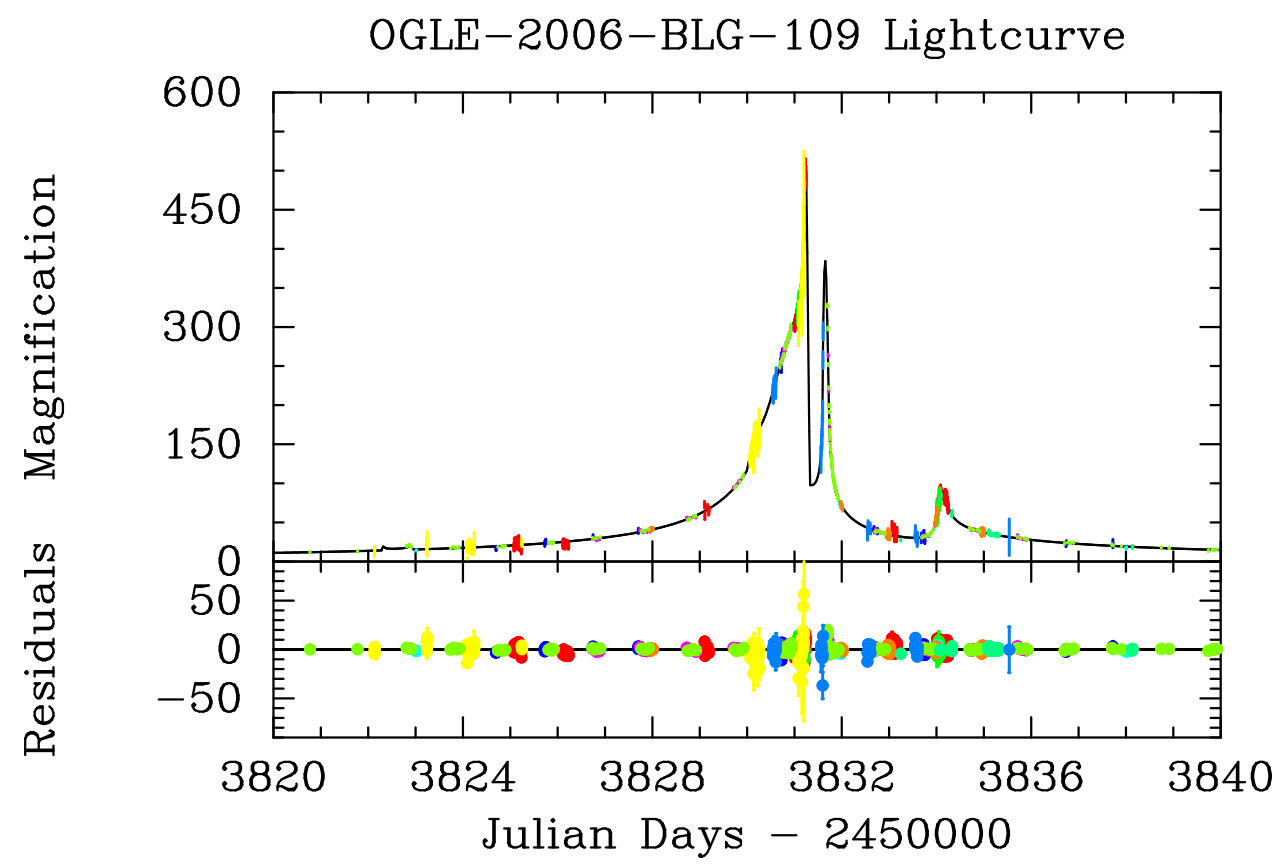

Figure 6.4: Model lightcurve for OGLE-2006-BLG-109, generated using the current version of mlens2.

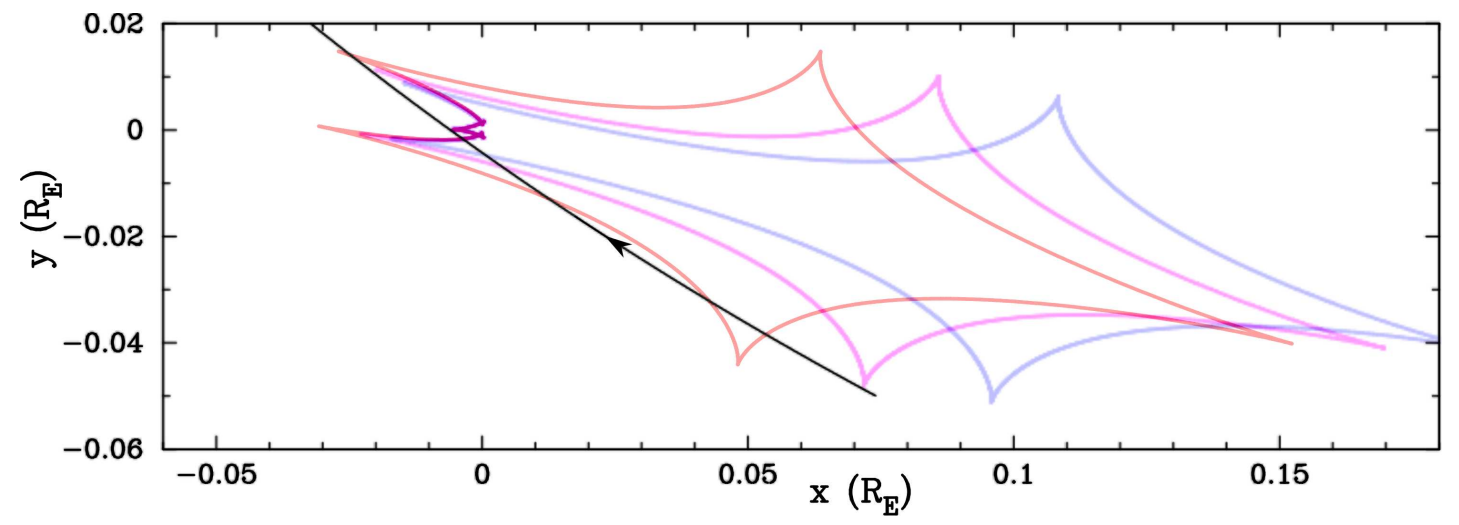

Figure 6.5: Caustic curves for MOA-2009-BLG-319, generated using the current version of mlens2, where the path and direction of the source track is shown by the black line and arrow. The red, magenta, and blue curves represent the critical curves at HJD' $=3820,3830$, and 3840 respectively, showing how the critical curves evolve as the lens system changes due to the lens motion in this event. 
mlens2 are preliminary data sets from late 2007, when the initial modelling of this event was done.

The control file and data sets used in Figure 6.4 were also run on the 2010 version of mlens2 and calculated a $\chi^{2}$ value of 2511.56 in 103 seconds. This demonstrates that the current version of this software is also about $9 \%$ faster than the earlier version of the software for this particular model.

Figure 6.6 shows two close-ups of the lightcurve presented in Figure 6.4. Both of these close-ups show times associated with the source crossing the caustic curves, at the times, $t \approx 3823$ and $t \approx 3831$, for Figures $6.6 \mathrm{a}$ and $6.6 \mathrm{~b}$ respectively. These plots show that the model lightcurve is slightly off from the observed data in some places. It has been suggested that the residuals between the model lightcurve and the observational data in Figure 6.6 might be systematic errors that were introduced when converting the parameters into the coordinate system used by mlens2 (Chote, 2011).

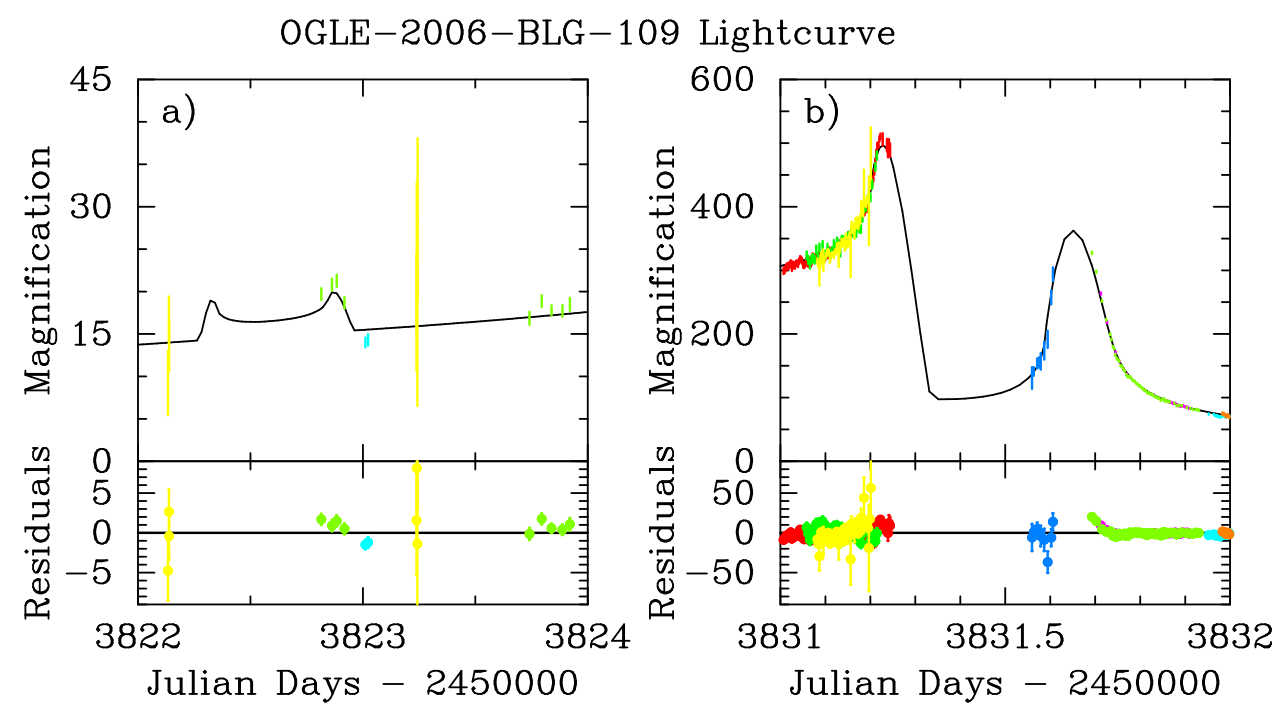

Figure 6.6: Close-up views of the model lightcurve shown in Figure 6.4, using the model given by Bennett et al. (2010). These views correspond to times associated with caustic crossings.

While the parameter search algorithms are not yet fully implemented in the mlens2 code, small grid searches can be performed by defining a range a values for one or more parameters, and calculating $\chi^{2}$ values for discrete parameter values at regular intervals in these parameter ranges. The ranges for these parameters can be defined in the control file.

A small grid search such as this was performed on the OGLE-2006-BLG-109 
data. This grid search only varied the five parameters relating to the source track and source size, and calculated the $\chi^{2}$ value for three values in each parameter range, giving a total of 243 model $\chi^{2}$ values. The control file for this grid search is presented in Appendix F, Figure F.3.

Out of these 243 models, the best fitting model had a $\chi^{2}$ value of 1799.76 , which was calculated in 87 seconds. The lightcurve of this model is given in Figures 6.7, and a close-up of this lightcurve around the caustic curve crossing is presented in Figure 6.8. While Figure 6.6 shows that Bennett's model deviates from the observed around HJD'=3831.7, this deviation is reduced in Figure 6.8, showing that latter model qualitatively fits slightly better with the observational data than Bennett's model. However, as mentioned before, these lightcurves only used the preliminary data sets from late 2007, not the final set of data used by Gaudi et al. (2008) or Bennett et al. (2010), so the quality of fit only applies to this preliminary set of data. The control file for this better fitting model is given in Appendix F, Figure F.4.

The model shown in Figure 6.7 is only the best fitting model out of the models tested; it is not the best fitting model out of all possible models. However, it shows that the model provided by Bennett et al. (2010) is not the optimal model for the preliminary OGLE-2006-BLG-109 data. When the parameter search algorithms are completely implemented in the mlens2 code, it is hoped that a full optimisation search could be run on this event to obtain the best fitting model in all parameter space. 


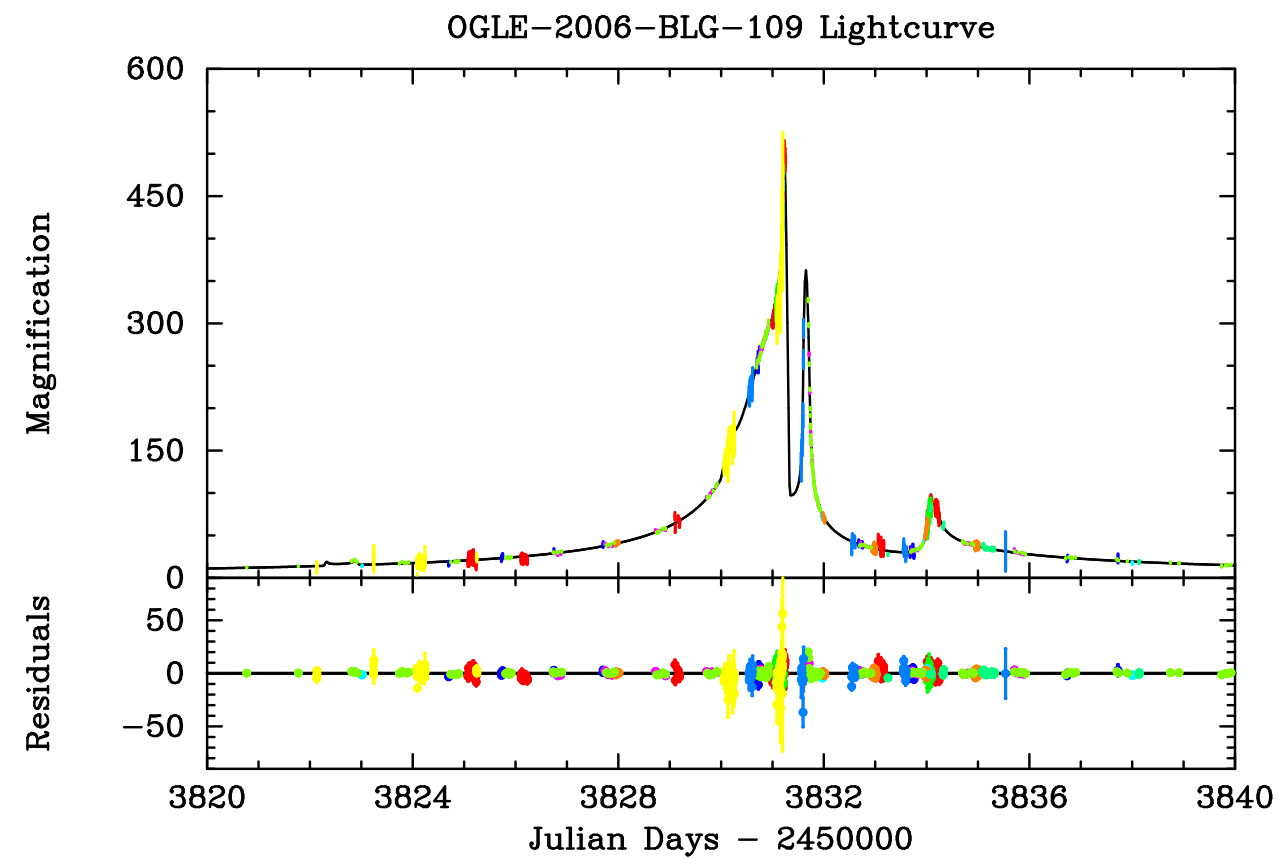

Figure 6.7: Best fitting model lightcurve for OGLE-2006-BLG-109, generated using the current version of mlens2.

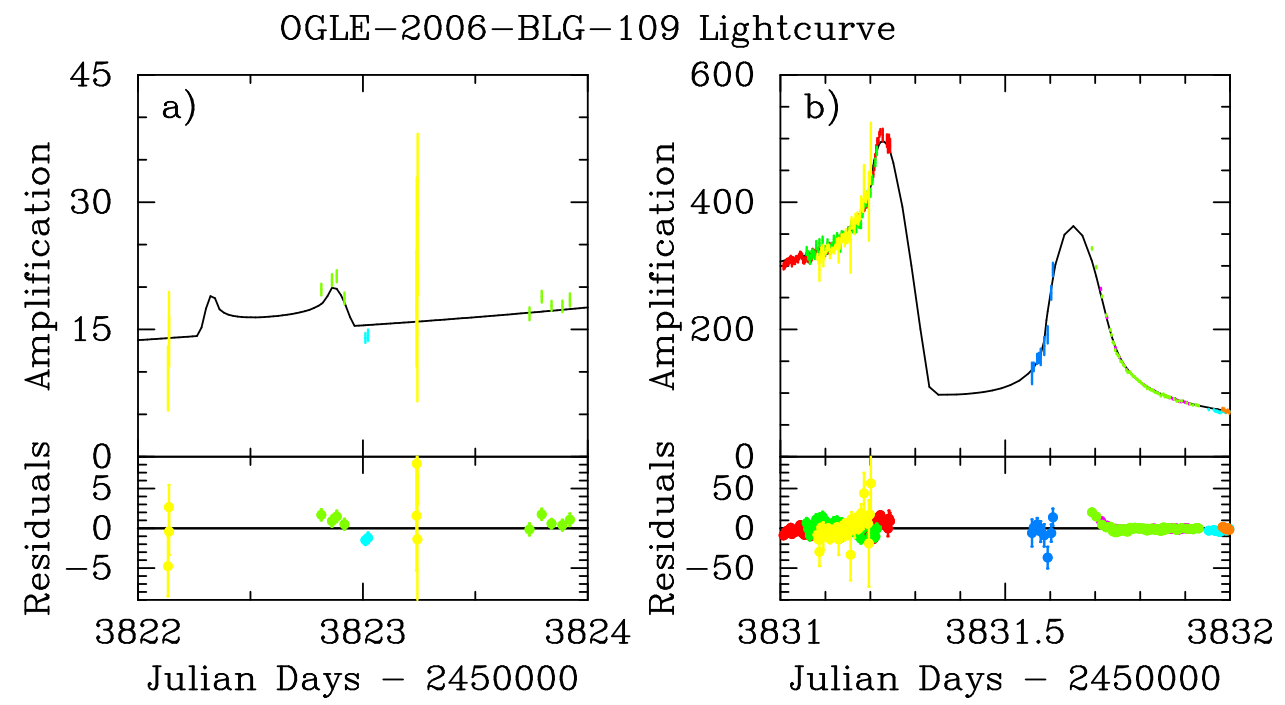

Figure 6.8: Close-up views of the model lightcurve shown in Figure 6.7. These views correspond to times associated with caustic crossings. 


\section{Chapter 7}

\section{Conclusions}

This thesis describes the improvements to the semi-analytic approach to modelling gravitational microlensing events. These improvements were primarily built on top of the semi-analytic approach developed by Chote (2011).

The initial step in the semi-analytic approach is to rearrange the lens equation into a lens polynomial, where the complex roots of the polynomial is a superset of the positions of the images. As part of this thesis project, we developed a method to rearrange the lens equations into the lens polynomials. We also developed a similar method to obtain a polynomial describing the critical curves. While this thesis did not explore lens systems with more than four lenses, the polynomials in this thesis are presented in a general form and can be used to describe theoretical lens systems with any number of lenses.

Using these polynomials, we showed that the lightcurve, critical curves and caustic curves exhibited an additive nature when the mass ratios were on the order of planetary mass ratios. This means that multiple planetary systems can be approximated using a number of binary lens systems (each consisting of the host star and a planet) added together. While this behaviour is only an approximation and cannot be used to accurately model observed lightcurves, it is useful to get a feel of the behaviour of the caustic curves and source magnification in multiple planetary systems.

To incorporate finite source effects into the code, we developed the multipole (quadrapole and hexadecapole) approximations. Unlike the polygon approximation, which requires a large number of source positions, the multipole approxi- 
mations require only a handful of source points, thus they are computationally much less expensive than the polygon method. However, it suffers some of the same problems as the point source magnification, such as diverging it crosses a caustic curve.

We also developed the 'natural' dynamic method to construct the source polygon for the polygon approximation. However, this method was found to be inefficient, as it used an order of magnitude more points than the dynamic method developed by Chote (2011).

Along with these approximations, we developed a successful algorithm to determine which approximation (point source, quadrapole, hexadecapole or polygon) was most suitable for finding the lens positions for a particular source position, and incorporated this algorithm and the approximations into the mlens2 code.

As part of this thesis work, several numerical techniques were investigated to find the optimal algorithms to solve the lens polynomials. While investigating these methods we found that inaccuracies arise in obtaining the correct image positions, particularly in the roots close to the planetary lenses. These inaccuracies in the root positions ultimately result in incorrect image magnification calculations and an inaccurate lightcurve.

We found that significant improvements could be obtained by developing the origin shifting technique, which shifted the origin to each lens before solving the polynomials. This technique dramatically reduced the inaccuracies in the roots but at a cost of increasing the time taken to calculate the roots.

We discovered that the Jenkins-Traub algorithm without polishing was the most accurate algorithm for calculating the roots. We also discovered that using the default initial values for these algorithms was slightly more accurate than using the roots from the previous source position as the initial values. Using the roots from the previous source position was faster than using the default initial values, but only when the source magnifications were solved sequentially from one end of the source track to the other, with short gaps between each adjacent source position. In the current version of the mlens2 code it was discovered that 
using the default initial values was actually faster than using the roots from the previous source position.

The 2010 version of the mlens2 software used Jenkins-Traub algorithm with polishing and with its default initial values but without origin shifting. Therefore, mlens2 was adapted by removing the polishing and implementing a process where the algorithm would "fall back" on origin shifting if the algorithm orignally failed to return the expected number of physical images or the expected total parity.

The new 2012 version of the mlens2 code proved to be much more robust and efficient than the old 2010 version. This was demonstrated in the lightcurves and the $\chi^{2}$ calculations for the microlensing events MOA-2009-BLG-319 and OGLE2006-BLG-109, with the latter event being a triple lens event. The software was also tested on some theoretical four lens events, showing that the software is more than capable for calculating lightcurve and $\chi^{2}$ values for multiple planet events with up to four lenses.

One important area of future research is the development of more methods to approximate the magnification of finite source sizes. Of particular interest is a method that calculates the magnification of a finite source (including limb darkening effects) using a relatively small number of source points, but will not return an infinite amplification when crossing caustic curves.

Another important area of future work will be the completion of the code for the parameter search for the MLserver executable. Once this has been achieved, the programme will then be implemented on the computing networks at VUW. In the future, running this executable will calculate $\chi^{2}$ values for millions of theoretical models to find the best-fit model for an observed microlensing event. This could potentially be used to model multiple planetary events in 'real time', as the events are being observed. 


\section{Appendix A}

\section{Derivation of Lens Polynomials}

The semi-analytic approach to gravitational microlensing events, as explained in Chapter 2, works by expressing the image positions as complex numbers $z=x+i y$ and rearranging the lens equation into a polynomial in terms of $z$. A configuration with $N$ lenses corresponds to lens polynomials of degree $N^{2}+1$. For multiple lens systems, the lens polynomial has a degree greater than 4 , so they cannot be solved analytically. Instead they are solved through numerical algorithms such as the Jenkins-Traub and Laguerre methods.

A basic overview of the lens polynomial was discussed in Section 2.4. This appendix presents a more detailed description and derivation of this polynomial for a general $N$ lens system. This derivation is partly based on a derivation for the 3 lens polynomial by Rhie (2002).

These expressions for the coefficients were incorporated into the mlens2 software package developed by the optical astronomy research group at Victoria University of Wellington. While the coefficients of the lens polynomials for a general $N$ lens case can be expressed mathematically, writing these general expressions into a code proved to be challenging. Therefore, the coefficient expressions specific to two, three, and four lenses were written into the code. These specific coefficient expressions for two and three lenses are expressed in this appendix. The full expressions of the coefficients for a four lens system are too lengthy to be expressed here, but can be easily obtained from the general expression presented in this appendix. 
The lens equation for a lens system with $N$ lenses can be written as,

$$
w=z-\sum_{j=1}^{N} \frac{\epsilon_{j}}{\bar{z}-\bar{r}_{j}},
$$

where the mass fraction of the $j$ th planet is represented by the real value $\epsilon_{j}$ and $\bar{r}_{j}$ is the complex conjugate of $r_{j}$, which is the 2-dimensional positions of the lens is represented by complex position. To eliminate the $\bar{z}$ terms, we take the complex conjugate of the lens equation and rearrange it to make $\bar{z}$ the subject, as follows,

$$
\bar{z}=\bar{w}+\sum_{j=1}^{N} \frac{\epsilon_{j}}{z-r_{j}} .
$$

By defining $z_{j}=z-r_{j}$, we can construct the polynomials $G$ and $H$ such that,

$$
\begin{aligned}
\sum_{k=0}^{N} G_{k} z^{k} & =G=\sum_{j=1}^{N} \epsilon_{j} \prod_{i \neq j} z_{i}, \\
\sum_{k=0}^{N} H_{k} z^{k} & =H=\prod_{i=1}^{N} z_{i} .
\end{aligned}
$$

The polynomial $H(z)$ has degree $N$ with its roots at the lens positions $r_{j}$, whereas the polynomial $G$ has degree $N-1$. The quotient of the polynomials $G$ and $H$ can be given as,

$$
\frac{G}{H}=\frac{\sum_{j=1}^{N} \epsilon_{j} \prod_{i \neq j} z_{i}}{\prod_{j=1}^{N} z_{j}}=\sum_{j=1}^{N} \frac{\epsilon_{j}}{z_{j}} .
$$

The conjugate of the three lens equation in Equation A.2 can then be expressed as,

$$
\bar{z}=\bar{w}+\frac{G}{H}
$$

To eliminate the $\bar{z}$ terms, Equation A.6 can be substituted into Equation A.1 to give,

$$
z-w=\sum_{j=1}^{N} \frac{\epsilon_{j}}{\frac{G}{H}+\bar{w}-\bar{r}_{j}} .
$$


Equation A.7 can be simplified by defining $\varpi_{j}=\bar{r}_{j}-\bar{w}$ giving,

$$
\begin{aligned}
z-w & =\sum_{j=1}^{N} \frac{\epsilon_{j}}{\frac{G}{H}-\varpi_{j}} \\
0 & =(z-w)-\sum_{j=1}^{N} \frac{H \epsilon_{j}}{G-H \varpi_{j}} \\
0 & =(z-w) \prod_{j=1}^{N}\left(G-\varpi_{j} H\right)-H \sum_{j=1}^{N}\left[\epsilon_{j} \prod_{j \neq i}\left(G-\varpi_{i} H\right)\right] .
\end{aligned}
$$

The Equation A.8 can be thought of as in two parts: the product $\prod_{j=1}^{N}(G-$ $\left.\varpi_{j} H\right)$ in the first half of the equation, and the $\operatorname{sum} \sum_{j=1}^{N}\left[\epsilon_{j} \prod_{j \neq i}\left(G-\varpi_{i} H\right)\right]$ in the second half of the equation. By defining $\pi_{j}=z-\varpi_{j}$, we can construct three polynomials $X, V$ and $W$, such that,

$$
\begin{aligned}
\sum_{i=0}^{N} X_{i} z^{i} & =X=\prod_{j=1}^{N} \pi_{j}, \\
\sum_{i=0}^{N} V_{i} z^{i} & =V=\sum_{j=1}^{N}\left[\epsilon_{j} \prod_{j \neq i} \pi_{j}\right], \\
\sum_{i=0}^{N} W_{i} z^{i} & =W=w X+V .
\end{aligned}
$$

We can consider product $\prod_{j=1}^{N}\left(G-\varpi_{j} H\right)$ can be considered analogous to polynomial $X$, while the sum $\sum_{j=1}^{N}\left[\epsilon_{j} \prod_{j \neq i}\left(G-\varpi_{i} H\right)\right]$ is analogous to the polynomial $V$. Therefore, using the coefficients of $X$ and $V$ we can rewrite Equation A.8 as,

$$
\begin{aligned}
0 & =(z-w)\left[\sum_{i=0}^{N} G^{i} H^{N-i} X^{i}\right]-\left[\sum_{i=0}^{N} G^{i} H^{N-i} V^{i}\right] \\
0 & =z \sum_{i=0}^{N} G^{i} H^{N-i} X^{i}-w \sum_{i=0}^{N} G^{i} H^{N-i} X^{i}-\sum_{i=0}^{N} G^{i} H^{N-i} V^{i} \\
0 & =z\left[\sum_{i=0}^{N} G^{i} H^{N-i} X^{i}\right]-\left[\sum_{i=0}^{N} G^{i} H^{N-i} W^{i}\right]
\end{aligned}
$$

Note that Equation A.12 contains the polynomials $G$ and $H$, where $H$ has a degree $N$ and $G$ has degree $N-1$. In the product $\prod_{j=1}^{N}\left(G-\varpi_{j} H\right), H$ is multiplied 
by itself at most $N$, giving $H^{N}$ a degree of $N^{2}$. This product is multiplied by $z$, giving a this equation a degree of $N^{2}+1$.

To obtain expressions for the coefficients of the polynomial in Equation A.12, we can rewrite the term $G^{i} H^{N-i}$ as,

$$
G^{j} H^{N-j}=\sum_{k=0}^{N^{2}} \eta_{j, k} z^{k},
$$

By substituting Equation A.13 into Equation A.12, the lens polynomial becomes,

$$
\begin{aligned}
0 & =z\left[\sum_{j=0}^{N} \sum_{k=0}^{N^{2}}\left(\eta_{j, k} z^{k} X^{j}\right)\right]-\left[\sum_{j=0}^{N} \sum_{k=0}^{N^{2}}\left(\eta_{j, k} z^{k} W^{j}\right)\right] \\
0 & =\left[\sum_{j=0}^{N} \sum_{k=0}^{N^{2}}\left(\eta_{j, k} z^{k+1} X^{j}\right)\right]-\left[\sum_{j=0}^{N} \sum_{k=0}^{N^{2}}\left(\eta_{j, k} z^{k} W^{j}\right)\right] \\
0 & =\sum_{k=0}^{N^{2}+1} z^{k}\left[\sum_{j=0}^{N}\left(\eta_{j, k-1} X^{j}\right)-\sum_{j=0}^{N}\left(\eta_{j, k} W^{j}\right)\right] .
\end{aligned}
$$

The polynomial shown in Equation A.14 has degree $N^{2}+1$. This polynomial can be written as,

$$
0=\sum_{n=0}^{N^{2}+1} c_{k} z^{k}
$$

where the coefficients for this polynomial are given by,

$$
c_{k}=\sum_{j=0}^{N}\left(\eta_{j, k-1} X_{j}-\eta_{j, k} W_{j}\right] .
$$

To generate a lightcurve for a particular microlensing model, a series of point sources are positioned on the source plane and the positions and magnifications of the images are calculated for each source position. While the coefficients for $X, V$, and $W$ are dependent of the source position $w$, the coefficients for $G$ and $H$ depend only on the positions and mass ratios of the lenses. Therefore, when generating a theoretical lightcurve for a microlensing model that does not have relative lens motion, the coefficients for the $G$ and $H$ do not need to be recalculated for every source position, thus saving computational time. However, if the model includes orbital motion, the position of the lenses $r_{j}$ change over time, so the coefficients for the $G$ and $H$ need to be recalculated for every source position. 


\section{A.1 Two Lens System - Degree 5 Lens Polyno- mial}

From Equation A.1, the lens equation for a two lens system can be expressed as,

$$
w=z-\frac{\epsilon_{1}}{\bar{z}-\bar{r}_{1}}+\frac{\epsilon_{2}}{\bar{z}-\bar{r}_{2}},
$$

where the mass fractions of the planets are represented by the real values $\epsilon_{1}$ and $\epsilon_{2}$, and the 2-dimensional positions of the two lenses are represented by complex positions $r_{1}$ and $r_{2}$ respectively. The complex positions of the roots can be obtained by solving the 5th degree polynomial,

$$
0=\sum_{k=0}^{5} c_{k} z^{k}=c_{5} z^{5}+c_{4} z^{4}+c_{3} z^{3}+c_{2} z^{2}+c_{1} z+c_{0}
$$

where coefficients $c_{n}$ for this polynomial are given by,

$$
c_{k}=\eta_{2, k-1} X_{2}-\eta_{1, k-1} X_{1}+\eta_{0, k-1} X_{0}-\left[\eta_{2, k} W_{2}-\eta_{1, k} W_{1}+\eta_{0, k} W_{0}\right]
$$

The values for $\eta_{i, k}$ in the two lens case are given as,

$$
\begin{aligned}
& \eta_{2,4}=G_{2}^{2} \\
& \eta_{2,3}=2 G_{1} G_{2}, \\
& \eta_{2,2}=G_{1}^{2}+2 G_{0} G_{2}, \\
& \eta_{2,1}=2 G_{0} G_{1} \\
& \eta_{2,0}=G_{0}^{2}
\end{aligned}
$$




$$
\begin{aligned}
& \eta_{1,4}=G_{2} H_{2}, \\
& \eta_{1,3}=G_{1} H_{2}+G_{2} H_{1}, \\
& \eta_{1,2}=G_{1} H_{1}+G_{0} H_{2}+H_{0} G_{2}, \\
& \eta_{1,1}=G_{0} H_{1}+G_{1} H_{0}, \\
& \eta_{1,0}=G_{0} H_{0} \\
& \eta_{0,4}=H_{2}^{2} \\
& \eta_{0,3}=2 H_{1} H_{2}, \\
& \eta_{0,2}=H_{1}^{2}+2 H_{0} H_{2}, \\
& \eta_{0,1}=2 H_{0} H_{1}, \\
& \eta_{0,0}=H_{0}^{2},
\end{aligned}
$$

where coefficients of the polynomials $G$ and $H$ can be given as,

$$
\begin{aligned}
& H_{2}=1, \\
& H_{1}=r_{1}+r_{2}, \\
& H_{0}=r_{1} r_{2}, \\
& G_{2}=0, \\
& G_{1}=\epsilon_{1}+\epsilon_{2}(=1), \\
& G_{0}=\epsilon_{1} r_{2}+\epsilon_{2} r_{1} .
\end{aligned}
$$

The values for $X_{j}, V_{j}$, and $W_{j}$ correspond to the coefficients of the polynomials $X, V$, and $W$, which are given as,

$$
\begin{aligned}
& X_{2}=1, \\
& X_{1}=\varpi_{1}+\varpi_{2}, \\
& X_{0}=\varpi_{1} \varpi_{2},
\end{aligned}
$$




$$
\begin{aligned}
V_{2} & =0, \\
V_{1} & =\epsilon_{1}+\epsilon_{2}(=1), \\
V_{0} & =\epsilon_{1} \varpi_{2}+\epsilon_{2} \varpi_{1}, \\
W_{2} & =w X_{2}+V_{2}(=w), \\
W_{1} & =w X_{1}+V_{1}, \\
W_{0} & =w X_{0}+V_{0} .
\end{aligned}
$$

where $\varpi_{1}=\overline{r_{1}}-\bar{w}$ and $\varpi_{2}=\overline{r_{2}}-\bar{w}$.

\section{A.2 Three Lens System - Degree 10 Lens Poly- nomial}

From Equation A.1, the lens equation for a three lens system can be expressed as,

$$
w=z-\frac{\epsilon_{1}}{\bar{z}-\bar{r}_{1}}+\frac{\epsilon_{2}}{\bar{z}-\bar{r}_{2}}+\frac{\epsilon_{3}}{\bar{z}-\bar{r}_{3}}
$$

where the mass fractions of the planets are represented by the real values $\epsilon_{1}$, $\epsilon_{2}$, and $\epsilon_{3}$, and the 2-dimensional positions of the three lenses are represented by complex positions $r_{1}, r_{2}$, and $r_{3}$ respectively. The complex positions of the roots can be obtained by solving a 10th degree polynomial, given as,

$$
\begin{aligned}
0 & =c_{10} z^{10}+c_{9} z^{9}+c_{8} z^{8}+c_{7} z^{7}+c_{6} z^{6}+c_{5} z^{5}+c_{4} z^{4}+c_{3} z^{3}+c_{2} z^{2}+c_{1} z+c_{0} \\
& =\sum_{k=0}^{10} c_{k} z^{k}
\end{aligned}
$$

The coefficients $c_{n}$ for this polynomial are given as,

$$
\begin{array}{r}
c_{k}=\eta_{3, k-1} X_{3}-\eta_{2, k-1} X_{2}+\eta_{1, k-1} X_{1}-\eta_{0, k-1} X_{0} \\
-\left[\eta_{3, k} W_{3}-\eta_{2, k} W_{2}+\eta_{1, k} W_{1}-\eta_{0, k} W_{0}\right] .
\end{array}
$$


The values for $\eta_{i, k}$ in the three lens case are given as,

$$
\begin{aligned}
& \eta_{3,9}=G_{3}^{3}, \\
& \eta_{3,8}=3 G_{2} G_{3}^{2}, \\
& \eta_{3,7}=3 G_{1} G_{3}^{2}+3 G_{2}^{2} G_{3}, \\
& \eta_{3,6}=G_{2}^{3}+3 G_{0} G_{3}^{2}+6 G_{1} G_{2} G_{3}, \\
& \eta_{3,5}=3 G_{1}^{2} G_{3}+3 G_{1} G_{2}^{2}+6 G_{0} G_{2} G_{3}, \\
& \eta_{3,4}=3 G_{1}^{2} G_{2}+3 G_{0} G_{2}^{2}+6 G_{0} G_{1} G_{3}, \\
& \eta_{3,3}=G_{1}^{3}+3 G_{0}^{2} G_{3}+6 G_{0} G_{1} G_{2}, \\
& \eta_{3,2}=3 G_{0} G_{1}^{2}+3 G_{0}^{2} G_{2}, \\
& \eta_{3,1}=3 G_{0}^{2} G_{1}, \\
& \eta_{3,0}=G_{0}^{3}, \\
& \eta_{2,9}=G_{3}^{2} H_{3}, \\
& \eta_{2,8}=2 G_{2} G_{3} H_{3}+G_{3}^{2} H_{2}, \\
& \eta_{2,7}=2 G_{1} G_{3} H_{3}+G_{2}^{2} H_{3}+2 G_{2} G_{3} H_{2}+G_{3}^{2} H_{1}, \\
& \eta_{2,6}=2 G_{0} G_{3} H_{3}+2 G_{1} G_{2} H_{3}+2 G_{1} G_{3} H_{2}+G_{2}^{2} H_{2} \\
& +2 G_{2} G_{3} H_{1}+G_{3}^{2} H_{0}, \\
& \eta_{2,5}=2 G_{0} G_{2} H_{3}+2 G_{0} G_{3} H_{2}+G_{1}^{2} H_{3}+2 G_{1} G_{2} H_{2} \\
& +2 G_{1} G_{3} H_{1}+G_{2}^{2} H_{1}+2 G_{2} G_{3} H_{0}, \\
& \eta_{2,4}=2 G_{0} G_{1} H_{3}+2 G_{0} G_{2} H_{2}+2 G_{0} G_{3} H_{1}+G_{1}^{2} H_{2} \\
& +2 G_{1} G_{2} H_{1}+2 G_{1} G_{3} H_{0}+G_{2}^{2} H_{0}, \\
& \eta_{2,3}=G_{0}^{2} H_{3}+2 G_{0} G_{1} H_{2}+2 G_{0} G_{2} H_{1}+2 G_{0} G_{3} H_{0} \\
& +G_{1}^{2} H_{1}+2 G_{1} G_{2} H_{0}, \\
& \eta_{2,2}=G_{0}^{2} H_{2}+2 G_{0} G_{1} H_{1}+2 G_{0} G_{2} H_{0}+G_{1}^{2} H_{0}, \\
& \eta_{2,1}=G_{0}^{2} H_{1}+2 G_{0} G_{1} H_{0}, \\
& \eta_{2,0}=G_{0}^{2} H_{0}
\end{aligned}
$$




$$
\begin{aligned}
& \eta_{1,9}=G_{3} H_{3}^{2}, \\
& \eta_{1,8}=G_{2} H_{3}^{2}+2 G_{3} H_{2} H_{3}, \\
& \eta_{1,7}=G_{1} H_{3}^{2}+2 G_{2} H_{2} H_{3}+2 G_{3} H_{1} H_{3}+G_{3} H_{2}^{2}, \\
& \eta_{1,6}=G_{0} H_{3}^{2}+2 G_{1} H_{2} H_{3}+2 G_{2} H_{1} H_{3}+G_{2} H_{2}^{2} \\
& +2 G_{3} H_{0} H_{3}+2 G_{3} H_{1} H_{2}, \\
& \eta_{1,5}=2 G_{0} H_{2} H_{3}+G_{1} H_{2}^{2}+2 G_{1} H_{1} H_{3}+2 G_{2} H_{0} H_{3} \\
& +2 G_{2} H_{1} H_{2}+2 G_{3} H_{0} H_{2}+G_{3} H_{1}^{2}, \\
& \eta_{1,4}=2 G_{0} H_{1} H_{3}+G_{0} H_{2}^{2}+2 G_{1} H_{0} H_{3}+2 G_{1} H_{1} H_{2} \\
& +2 G_{2} H_{0} H_{2}+G_{2} H_{1}^{2}+2 G_{3} H_{0} H_{1}, \\
& \eta_{1,3}=2 G_{0} H_{0} H_{3}+2 G_{0} H_{1} H_{2}+2 G_{1} H_{0} H_{2}+G_{1} H_{1}^{2} \\
& +2 G_{2} H_{0} H_{1}+G_{3} H_{0}^{2}, \\
& \eta_{1,2}=G_{0} H_{1}^{2}+2 G_{0} H_{0} H_{2}+2 G_{1} H_{0} H_{1}+G_{2} H_{0}^{2}, \\
& \eta_{1,1}=2 G_{0} H_{0} H_{1}+G_{1} H_{0}^{2}, \\
& \eta_{1,0}=G_{0} H_{0}^{2}, \\
& \eta_{0,9}=H_{3}^{3}, \\
& \eta_{0,8}=3 \mathrm{H}_{2} \mathrm{H}_{3}^{2}, \\
& \eta_{0,7}=3 H_{1} H_{3}^{2}+3 H_{2}^{2} H_{3}, \\
& \eta_{0,6}=H_{2}^{3}+3 H_{0} H_{3}^{2}+6 H_{1} H_{2} H_{3}, \\
& \eta_{0,5}=3 H_{1}^{2} H_{3}+3 H_{1} H_{2}^{2}+6 H_{0} H_{2} H_{3}, \\
& \eta_{0,4}=3 H_{1}^{2} H_{2}+3 H_{0} H_{2}^{2}+6 H_{0} H_{1} H_{3}, \\
& \eta_{0,3}=H_{1}^{3}+3 H_{0}^{2} H_{3}+6 H_{0} H_{1} H_{2}, \\
& \eta_{0,2}=3 H_{0} H_{1}^{2}+3 H_{0}^{2} H_{2}, \\
& \eta_{0,1}=3 H_{0}^{2} H_{1}, \\
& \eta_{0,0}=H_{0}^{3} .
\end{aligned}
$$


where coefficients of the polynomials $G$ and $H$ can be given by,

$$
\begin{aligned}
& H_{3}=1, \\
& H_{2}=r_{1}+r_{2}+r_{3}, \\
& H_{1}=r_{1} r_{2}+r_{1} r_{3}+r_{2} r_{3}, \\
& H_{0}=r_{1} r_{2} r_{3}, \\
& G_{3}=0, \\
& G_{2}=\epsilon_{1}+\epsilon_{2}+\epsilon_{3}(=1), \\
& G_{1}=\epsilon_{1}\left(r_{2}+r_{3}\right)+\epsilon_{2}\left(r_{1}+r_{3}\right)+\epsilon_{3}\left(r_{1}+r_{2}\right), \\
& G_{0}=\epsilon_{1} r_{2} r_{3}+\epsilon_{2} r_{1} r_{3}+\epsilon_{3} r_{1} r_{2} .
\end{aligned}
$$

The values for $X_{j}, V_{j}$, and $W_{j}$ correspond to the coefficients of the polynomials $X, V$, and $W$, which are given as,

$$
\begin{aligned}
X_{3} & =1 \\
X_{2} & =\varpi_{1}+\varpi_{2}+\varpi_{3} \\
X_{1} & =\varpi_{1} \varpi_{2}+\varpi_{1} \varpi_{3}+\varpi_{2} \varpi_{3} \\
X_{0} & =\varpi_{1} \varpi_{2} \varpi_{3} \\
V_{3} & =0 \\
V_{2} & =\epsilon_{1}+\epsilon_{2}+\epsilon_{3}(=1) \\
V_{1} & =\epsilon_{1}\left(\varpi_{2}+\varpi_{3}\right)+\epsilon_{2}\left(\varpi_{1}+\varpi_{3}\right)+\epsilon_{3}\left(\varpi_{1}+\varpi_{2}\right) \\
V_{1} & =\epsilon_{1} \varpi_{2} \varpi_{3}+\epsilon_{2} \varpi_{1} \varpi_{3}+\epsilon_{3} \varpi_{1} \varpi_{2} \\
W_{3} & =w X_{3}+V_{3}(=w) \\
W_{2} & =w X_{2}+V_{2} \\
W_{1} & =w X_{1}+V_{1} \\
W_{0} & =w X_{0}+V_{0}
\end{aligned}
$$

where $\varpi_{1}=\overline{r_{1}}-\bar{w}, \varpi_{2}=\overline{r_{2}}-\bar{w}$, and $\varpi_{3}=\overline{r_{3}}-\bar{w}$. 


\section{A.3 Solutions as the Source Position goes to In- finity}

To determine the behaviour of the lens equation solutions when the source star is far from the lens positions, we can apply the limit $w \rightarrow \infty$ to the lens polynomial. By applying this limit to Equation A.8, while dividing both sides by $\bar{w}^{N}$, we get,

$$
\begin{aligned}
\lim _{w \rightarrow \infty} \frac{0}{\bar{w}^{N}} & =\lim _{w \rightarrow \infty} \frac{(z-w) \prod_{j=1}^{N}\left(G-\varpi_{j} H\right)-H \sum_{j=1}^{N}\left[\epsilon_{j} \prod_{j \neq i}\left(G-\varpi_{i} H\right)\right]}{\bar{w}^{N}} \\
\lim _{w \rightarrow \infty} 0 & =\lim _{w \rightarrow \infty}(z-w) \prod_{j=1}^{N} \frac{\left(G-\left(\bar{r}_{j}-\bar{w}\right) H\right)}{\bar{w}}-\frac{H}{\bar{w}^{N}} \sum_{j=1}^{N}\left[\epsilon_{j} \prod_{j \neq i}\left(G-\varpi_{i} H\right)\right] \\
\lim _{w \rightarrow \infty} 0 & =\lim _{w \rightarrow \infty}(z-w) \prod_{j=1}^{N}\left(\frac{G}{\bar{w}}+\frac{\bar{r}_{j} H}{\bar{w}}-H\right)-\frac{H}{\bar{w}^{N}} \sum_{j=1}^{N}\left[\epsilon_{j} \prod_{j \neq i}\left(G-\varpi_{i} H\right)\right] \\
0 & =(z-w) \prod_{j=1}^{N}(H) \\
0 & =(z-w) H^{N} \\
0 & =(z-w) \prod_{j=1}^{N}\left(z-r_{j}\right)^{N} .
\end{aligned}
$$

Therefore, when the source is far from the lenses, there is one root near the source, $w$, and $N$ roots near each lens position, $r_{j}$. 


\section{Appendix B}

\section{The Jacobian}

The Jacobian matrix is a matrix in vector calculus that generalises the concept of the vector gradient $\nabla$ to a vector-valued function. This matrix contains the firstorder partial derivatives of the vector-valued function with respect to another vector. If $w(z)$ is a function from $n$-dimensional Euclidean space to $m$-dimensional Euclidean space, then this function can be expressed as the real-valued component functions $w_{1}\left(z_{1}, \ldots, z_{n}\right), \ldots w_{m}\left(z_{1}, \ldots, z_{n}\right)$. The Jacobian matrix $J_{M}$ of this function is given by,

$$
J_{M}=\frac{\partial\left(w_{1}, \ldots, w_{m}\right)}{\partial\left(z_{1}, \ldots, z_{n}\right)}=\left[\begin{array}{ccc}
\frac{\partial w_{1}}{\partial z_{1}} & \cdots & \frac{\partial w_{1}}{\partial z_{n}} \\
\vdots & \ddots & \vdots \\
\frac{\partial w_{m}}{\partial z_{1}} & \cdots & \frac{\partial w_{m}}{\partial z_{n}}
\end{array}\right]
$$

The Jacobian is particularly useful when we want to make a change of variables. For example, suppose we want to integrate a scalar-valued function $f\left(w_{1}, \ldots, w_{n}\right)$ over an $n$-dimensional surface,

$$
\int_{S} f\left(w_{1}, \ldots, w_{n}\right) d w_{1} \cdots d w_{n}
$$

If we wish to change the coordinates from $\left(w_{1}, \ldots, w_{m}\right)$ to $\left(z_{1}, \ldots, z_{n}\right)$, we transform between coordinates by writing each $w_{i}$ as a function of $\left(z_{1}, \ldots, z_{n}\right)$, such that,

$$
\begin{gathered}
w_{1}\left(z_{1}, \cdots, z_{n}\right), \\
\vdots \\
w_{m}\left(z_{1}, \cdots, z_{n}\right) .
\end{gathered}
$$


By differentiating these functions, we obtain the following expressions,

$$
\begin{aligned}
d w_{1} & =\frac{\partial w_{1}}{\partial z_{1}} d z_{1}+\cdots+\frac{\partial w_{1}}{\partial z_{n}} d z_{n} \\
& \vdots \\
d w_{m} & =\frac{\partial w_{m}}{\partial z_{1}} d z_{1}+\cdots+\frac{\partial w_{m}}{\partial z_{n}} d z_{n}
\end{aligned}
$$

Equations B.3 - B.3 can also be expressed as a product of a matrix and vector,

$$
\left[\begin{array}{c}
d w_{1} \\
\vdots \\
d w_{m}
\end{array}\right]=J_{M}\left[\begin{array}{c}
d z_{1} \\
\vdots \\
d z_{n}
\end{array}\right]
$$

where $J_{M}$ is the Jacobian identical to the one presented in Equation B.1.

The Jacobian determinant $J$ (often simply called the Jacobian) is the determinant of the square Jacobian matrix $J_{M}$, and is expressed as,

$$
J=\left|\frac{\partial\left(w_{1}, \ldots, w_{n}\right)}{\partial\left(z_{1}, \ldots, z_{n}\right)}\right|=\left|\begin{array}{ccc}
\frac{\partial w_{1}}{\partial z_{1}} & \cdots & \frac{\partial w_{1}}{\partial z_{n}} \\
\vdots & \ddots & \vdots \\
\frac{\partial w_{n}}{\partial z_{1}} & \cdots & \frac{\partial w_{n}}{\partial z_{n}}
\end{array}\right| .
$$

When transforming between coordinates, the Jacobian determinant is used to describe the ratio of an infinitesimal area element in the new coordinate system to an infinitesimal area element in the old coordinate system,

$$
J=\frac{d w_{1} \ldots d w_{n}}{d z_{1} \ldots d z_{n}}
$$

In this way, the Jacobian can be used to transform the function $f\left(w_{1}, \ldots, w_{n}\right)$ from the old coordinate system to the new coordinate system. For example, by substituting the Jacobian $J$ into Equation B.2, the integral can be expressed as,

$$
\int_{S} f\left(z_{1}, \ldots, z_{n}\right) J d z_{1} \cdots d z_{n}
$$

\section{B.1 Complex Coordinates}

In the semi-analytical approach to gravitational microlensing, the points on the source and image planes are represented as the complex numbers $w=u+v i$ 
and $z=x+y i$ respectively. The lens equation and its complex conjugate for a multiple lens system, which describe a transformation from the image to source plane, can be expressed respectively as,

$$
\begin{aligned}
& w=z-\sum_{j=0}^{N} \frac{\epsilon_{j}}{\bar{z}-\bar{r}_{j}}, \\
& \bar{w}=\bar{z}-\sum_{j=0}^{N} \frac{\epsilon_{j}}{z-r_{j}} .
\end{aligned}
$$

The Jacobian $J$ of the lens equation therefore describes the ratio of an infinitesimal area on the source plane to an infinitesimal area on the image plane,

$$
J=\left|\begin{array}{ll}
\frac{\partial u}{\partial x} & \frac{\partial u}{\partial y} \\
\frac{\partial v}{\partial x} & \frac{\partial v}{\partial y}
\end{array}\right|=\frac{\partial u}{\partial x} \frac{\partial v}{\partial y}-\frac{\partial u}{\partial y} \frac{\partial v}{\partial x} .
$$

Therefore, the image magnification (the ratio of the area of the image to the area of the source) can be described as the reciprocal of the Jacobian determinant $J^{-1}$. Since the lens equation is described in terms of $w, z, \bar{w}$, and $\bar{z}$, where the complex conjugates are given by $\bar{w}=u-v i$ and $\bar{z}=x-y i$. Therefore, it is more convenient and more powerful mathematically to express the Jacobian using the terms $w, z, \bar{w}$, and $\bar{z}$.

To do this, the first step is to express the $w$ components $u$ and $v$ in terms of $w$ and $\bar{w}$, as follows,

$$
u=\frac{w+\bar{w}}{2} \quad v=\frac{w-\bar{w}}{2 i} .
$$

The next step is to obtain the appropriate first-order partial derivatives for $z, \bar{z}, u$ and $v$,

$$
\begin{array}{ll}
\frac{\partial z}{\partial x}=1, & \frac{\partial z}{\partial y}=i, \\
\frac{\partial \bar{z}}{\partial x}=1, & \frac{\partial \bar{z}}{\partial y}=-i,
\end{array}
$$

and

$$
\begin{aligned}
\frac{\partial u}{\partial w} & =\frac{1}{2}, & \frac{\partial u}{\partial \bar{w}} & =\frac{1}{2 i}, \\
\frac{\partial v}{\partial w} & =\frac{1}{2}, & \frac{\partial v}{\partial \bar{w}} & =\frac{-1}{2 i} .
\end{aligned}
$$


From the above expressions, the Jacobian can be expressed in terms of $w, z$, $\bar{w}$ and $\bar{z}$ by applying the chain rule to Equation B.7 to give,

$$
\begin{aligned}
& \left|\begin{array}{ll}
\frac{\partial u}{\partial x} & \frac{\partial u}{\partial y} \\
\frac{\partial v}{\partial x} & \frac{\partial v}{\partial y}
\end{array}\right|=\frac{\partial u}{\partial x} \frac{\partial v}{\partial y}-\frac{\partial v}{\partial x} \frac{\partial u}{\partial y} \\
& =\left(\frac{\partial u}{\partial w} \frac{\partial w}{\partial x}+\frac{\partial u}{\partial \bar{w}} \frac{\partial \bar{w}}{\partial x}\right)\left(\frac{\partial v}{\partial w} \frac{\partial w}{\partial y}+\frac{\partial v}{\partial \bar{w}} \frac{\partial \bar{w}}{\partial y}\right) \\
& -\left(\frac{\partial v}{\partial w} \frac{\partial w}{\partial x}+\frac{\partial v}{\partial \bar{w}} \frac{\partial \bar{w}}{\partial x}\right)\left(\frac{\partial u}{\partial w} \frac{\partial w}{\partial y}+\frac{\partial u}{\partial \bar{w}} \frac{\partial \bar{w}}{\partial y}\right) \\
& =\left(\frac{1}{2} \frac{\partial w}{\partial x}+\frac{1}{2 i} \frac{\partial \bar{w}}{\partial x}\right)\left(\frac{1}{2} \frac{\partial w}{\partial y}+\frac{-1}{2 i} \frac{\partial \bar{w}}{\partial y}\right) \\
& -\left(\frac{1}{2} \frac{\partial w}{\partial x}+\frac{-1}{2 i} \frac{\partial \bar{w}}{\partial x}\right)\left(\frac{1}{2} \frac{\partial w}{\partial y}+\frac{1}{2 i} \frac{\partial \bar{w}}{\partial y}\right) \\
& =\frac{1}{2 i}\left[\frac{\partial \bar{w}}{\partial x} \frac{\partial w}{\partial y}-\frac{\partial w}{\partial x} \frac{\partial \bar{w}}{\partial y}\right] \\
& =\frac{1}{2 i}\left[\left(\frac{\partial \bar{w}}{\partial z} \frac{\partial z}{\partial x}+\frac{\partial \bar{w}}{\partial \bar{z}} \frac{\partial \bar{z}}{\partial x}\right)\left(\frac{\partial w}{\partial z} \frac{\partial z}{\partial y}+\frac{\partial w}{\partial \bar{z}} \frac{\partial \bar{z}}{\partial y}\right)\right. \\
& \left.+\left(\frac{\partial w}{\partial z} \frac{\partial z}{\partial x}+\frac{\partial w}{\partial \bar{z}} \frac{\partial \bar{z}}{\partial x}\right)\left(\frac{\partial \bar{w}}{\partial z} \frac{\partial z}{\partial y}+\frac{\partial \bar{w}}{\partial \bar{z}} \frac{\partial \bar{z}}{\partial y}\right)\right] \\
& =\frac{1}{2 i}\left[\left(\frac{\partial \bar{w}}{\partial z}+\frac{\partial \bar{w}}{\partial \bar{z}}\right)\left(i \frac{\partial w}{\partial z}-i \frac{\partial w}{\partial \bar{z}}\right)-\left(\frac{\partial w}{\partial z}+\frac{\partial w}{\partial \bar{z}}\right)\left(i \frac{\partial \bar{w}}{\partial z}-i \frac{\partial \bar{w}}{\partial \bar{z}}\right)\right] \\
& =\frac{\partial w}{\partial z} \frac{\partial \bar{w}}{\partial \bar{z}}-\frac{\partial w}{\partial \bar{z}} \frac{\partial \bar{w}}{\partial z} \\
& =\left|\begin{array}{ll}
\frac{\partial w}{\partial z} & \frac{\partial w}{\partial \bar{z}} \\
\frac{\partial \bar{w}}{\partial z} & \frac{\partial \bar{w}}{\partial \bar{z}}
\end{array}\right| .
\end{aligned}
$$

By differentiating Equation B.7 and B.7, the first-order partial derivatives are given by,

$$
\begin{aligned}
\frac{\partial w}{\partial z}=1, & \frac{\partial w}{\partial \bar{z}} & =\sum_{j=0}^{N} \frac{\epsilon_{j}}{\left(\bar{z}-\bar{r}_{j}\right)^{2}}, \\
\frac{\partial \bar{w}}{\partial z}=\sum_{j=0}^{N} \frac{\epsilon_{j}}{\left(z-r_{j}\right)^{2}}, & \frac{\partial \bar{w}}{\partial \bar{z}} & =1 .
\end{aligned}
$$

When the above derivatives are substituted into Equation B.8, the Jacobian can be expressed as,

$$
J=\left|\begin{array}{cc}
1 & \frac{\partial w}{\partial \bar{z}} \\
\frac{\partial \bar{w}}{\partial z} & 1
\end{array}\right|=1-\frac{\partial w}{\partial \bar{z}} \frac{\partial \bar{w}}{\partial z}
$$




\section{B.2 Derivative of the Jacobian}

The derivatives of the Jacobian with respect to $z$ and $\bar{z}$ can be used to describe the rate of change of the image magnification across the image plane. These derivatives are especially useful when deriving the multipole approximations in Appendix D. Since the Jacobian in Equation B.11 depends only on $\partial w / \partial \bar{z}$ and $\partial \bar{w} / \partial z$, these derivatives need to be obtained before calculating the Jacobian derivatives. Note that $\partial w / \partial \bar{z}$ is independent of $z$, and $\partial \bar{w} / \partial z$ is independent of $\bar{z}$, so

$$
\begin{aligned}
& \frac{\partial^{2} w}{\partial z \partial \bar{z}}=0, \\
& \frac{\partial^{2} \bar{w}}{\partial \bar{z} \partial z}=0 .
\end{aligned}
$$

Therefore, the non-zero derivatives can be expressed as,

$$
\begin{aligned}
\frac{\partial^{2} \bar{w}}{\partial z^{2}} & =-\sum_{j=0}^{N} \frac{2 \epsilon_{j}}{\left(z-r_{j}\right)^{3}}, \\
\frac{\partial^{3} \bar{w}}{\partial z^{3}} & =\sum_{j=0}^{N} \frac{6 \epsilon_{j}}{\left(z-r_{j}\right)^{4}}, \\
\frac{\partial^{p+1} \bar{w}}{\partial z^{p+1}} & =(-1)^{p} \sum_{j=0}^{N} \frac{(p+1) ! \epsilon_{j}}{\left(z-r_{j}\right)^{p+2}}
\end{aligned}
$$

and

$$
\begin{aligned}
\frac{\partial^{2} w}{\partial \bar{z}^{2}} & =-\sum_{j=0}^{N} \frac{2 \epsilon_{j}}{\left(\bar{z}-\bar{r}_{j}\right)^{3}}, \\
\frac{\partial^{3} w}{\partial \bar{z}^{3}} & =\sum_{j=0}^{N} \frac{6 \epsilon_{j}}{\left(\bar{z}-\overline{r_{j}}\right)^{4}}, \\
\frac{\partial^{q+1} w}{\partial \bar{z}^{q+1}} & =(-1)^{q} \sum_{j=0}^{N} \frac{(q+1) ! \epsilon_{j}}{\left(z-r_{j}\right)^{q+2}} .
\end{aligned}
$$

The partial derivatives of the Jacobian can be obtained by differentiating 
Equation B.11 by $z$ and $\bar{z}$, which gives,

$$
\begin{aligned}
\frac{\partial J}{\partial z} & =\frac{\partial^{2} \bar{w}}{\partial z^{2}} \frac{\partial w}{\partial \bar{z}} \\
\frac{\partial J}{\partial \bar{z}} & =\frac{\partial \bar{w}}{\partial z} \frac{\partial^{2} w}{\partial \bar{z}^{2}} \\
\frac{\partial J^{2}}{\partial z^{2}} & =\frac{\partial^{3} \bar{w}}{\partial z^{3}} \frac{\partial w}{\partial \bar{z}} \\
\frac{\partial J^{2}}{\partial z \partial \bar{z}} & =\frac{\partial^{2} \bar{w}}{\partial^{2} z} \frac{\partial^{2} w}{\partial \bar{z}^{2}}, \\
\frac{\partial J^{2}}{\partial \bar{z}^{2}} & =\frac{\partial \bar{w}}{\partial z} \frac{\partial^{3} w}{\partial \bar{z}^{3}} \\
\frac{\partial J^{p+q}}{\partial z^{p} \partial \bar{z}^{q}} & =\frac{\partial^{p+1} \bar{w}}{\partial z^{p+1}} \frac{\partial^{q+1} w}{\partial \bar{z}^{q+1}} .
\end{aligned}
$$

\section{B.3 Derivative of the Magnification}

The Jacobian $J$ of the lens equation describes the ratio of an infinitesimal area element on the source plane to an infinitesimal area element on the image plane. The absolute value of this area ratio for each image is defined as the magnification, $\mu$, of the image and can be calculated from the inverse of the absolute Jacobian determinant,

$$
\mu=\frac{1}{|J|}
$$

The total magnification $A$ of a point source can therefore be defined as the sum of all the image magnifications, $\mu$, such that,

$$
A=\sum_{\text {images }} \mu=\sum_{\text {images }} \frac{1}{|J|}=\sum_{\text {images }} \frac{\operatorname{sgn}(J)}{J} \quad(J \neq 0) .
$$

where $\operatorname{sgn}(J)$ is the sign of $J$, i.e.,

$$
\begin{array}{r}
\operatorname{sgn}(J)=+1 \quad \text { if } \quad J>0, \\
\operatorname{sgn}(J)=0 \quad \text { if } \quad J=0, \\
\operatorname{sgn}(J)=-1 \quad \text { if } \quad J<0 .
\end{array}
$$


The derivatives of the image magnification can be obtained using Faà di Bruno's formula (Faà di Bruno, 1855), which generalises the concept of the chain rule to higher derivatives,

$$
\begin{aligned}
& \frac{\partial^{2} \mu(J(z, \bar{z}))}{\partial z \partial \bar{z}}=\frac{\partial^{2} \mu}{\partial J^{2}} \frac{\partial J}{\partial z} \frac{\partial J}{\partial \bar{z}}+\frac{\partial \mu}{\partial J} \frac{\partial^{2} J}{\partial z \partial \bar{z}}, \\
\frac{\partial^{4} \mu(J(z, \bar{z}))}{\partial z^{2} \partial \bar{z}^{2}}= & \frac{\partial^{4} \mu}{\partial J^{4}}\left(\frac{\partial J}{\partial z}\right)^{2}\left(\frac{\partial J}{\partial \bar{z}}\right)^{2} \\
& +\frac{\partial^{3} \mu}{\partial J^{3}}\left[\left(\frac{\partial J}{\partial z}\right)^{2} \frac{\partial^{2} J}{\partial \bar{z}^{2}}+4 \frac{\partial J}{\partial z} \frac{\partial J}{\partial \bar{z}} \frac{\partial^{2} J}{\partial z \partial \bar{z}}+\frac{\partial^{2} J}{\partial z^{2}}\left(\frac{\partial J}{\partial \bar{z}}\right)^{2}\right] \\
& +\frac{\partial^{2} \mu}{\partial J^{2}}\left[2 \frac{\partial J}{\partial z} \frac{\partial^{3} J}{\partial z \partial^{2} \bar{z}}+2 \frac{\partial^{3} J}{\partial^{2} z \partial \bar{z}} \frac{\partial J}{\partial \bar{z}}+2\left(\frac{\partial^{2} J}{\partial z \partial \bar{z}}\right)^{2}+\frac{\partial^{2} J}{\partial z^{2}} \frac{\partial^{2} J}{\partial \bar{z}^{2}}\right] \\
& +\frac{\partial \mu}{\partial J} \frac{\partial^{4} J}{\partial z^{2} \partial \bar{z}^{2}} .
\end{aligned}
$$

where the derivatives of the Jacobian are given in Equations B.14 - B.19. Similar expression for higher derivatives can also be obtained using Faà di Bruno's formula. The derivatives of the magnification with respect to the Jacobian (when $J \neq 0)$ can be expressed as,

$$
\begin{aligned}
\mu & =\frac{\operatorname{sgn}(J)}{J}, \\
\frac{\partial \mu}{\partial J} & =\frac{-\operatorname{sgn}(J)}{J^{2}}, \\
\frac{\partial^{2} \mu}{\partial J^{2}} & =\frac{2 \operatorname{sgn}(J)}{J^{3}}, \\
\frac{\partial^{3} \mu}{\partial J^{3}} & =\frac{-6 \operatorname{sgn}(J)}{J^{4}}, \\
\frac{\partial^{4} \mu}{\partial J^{4}} & =\frac{24 \operatorname{sgn}(J)}{J^{5}}, \\
\frac{\partial^{k} \mu}{\partial J^{k}} & =\frac{(-1)^{k} k ! \operatorname{sgn}(J)}{J^{k+1}} .
\end{aligned}
$$




\section{Appendix $\mathrm{C}$}

\section{Derivation of Critical Curve Polynomials}

As mentioned in Section 3.3, the critical curves in gravitational microlensing are defined as the loci of points on the image plane where a point image has an infinite magnification. In a similar way to the lens polynomials and the positions of the images in Appendix A, the points that describe the critical curves can be given as solutions to polynomials. These critical polynomials can be derived using the same nomenclature described in Appendix A and like the lens polynomials, the critical curve polynomials are derived by expressing the image positions as complex numbers $z=x+i y$. However, although a lens system with $N$ lenses corresponds to a lens polynomial of degree $N^{2}+1$, the critical curve polynomial for an $N$ lens system has a degree $2 N$.

This appendix discusses and derives the critical curve polynomial for a general $N$ lens system. The expressions for the coefficients of the two and three lens cases are also given in this appendix. These polynomials can then be solved through numerical algorithms such as the Jenkins-Traub and Laguerre methods.

In Section 3.3, we mentioned that the critical curves correspond to the points on the image plane where the Jacobian of the lens equation is zero. As demonstrated in Appendix B, Section B.1, the Jacobian of the lens equation is expressed as,

$$
J=\left|\begin{array}{ll}
\frac{\partial w}{\partial z} & \frac{\partial w}{\partial \bar{z}} \\
\frac{\partial \bar{w}}{\partial z} & \frac{\partial \bar{w}}{\partial \bar{z}}
\end{array}\right|=\left|\begin{array}{cc}
1 & \kappa(\bar{z}) \\
\kappa(z) & 1
\end{array}\right|=1-|\kappa(z)|^{2},
$$


where $\kappa$ is defined as the potential flow on the image plane and is given as,

$$
\kappa=\frac{\partial \bar{w}}{\partial z} .
$$

Therefore, the critical curves are the loci of points where,

$$
\begin{aligned}
J & =1-|\kappa(z)|^{2} \\
0 & =1-|\kappa(z)|^{2} \\
|\kappa(z)|^{2} & =1 \\
\kappa(z) & =e^{i \phi},
\end{aligned}
$$

where $\phi$ is any angle between 0 and $2 \pi$. The loci of points that describe the critical curves can be obtained by solving Equation C.3 for these values of $\phi$. Configurations with 2,3 , and $N$ lenses correspond to critical curve polynomials of degree 4,6 , and $2 N$ respectively.

The lens equation and its complex conjugate for a two lens system are given by

$$
\begin{aligned}
& w=z-\sum_{j=1}^{N} \frac{\epsilon_{j}}{\bar{z}-\bar{r}_{j}}, \\
& \bar{w}=\bar{z}-\sum_{j=1}^{N} \frac{\epsilon_{j}}{z-r_{j}},
\end{aligned}
$$

where the mass fractions of the planets are represented by the (real) values $\epsilon_{j}$, and the 2-dimensional positions of the two lenses are represented by the complex positions $r_{j}$. We can simplify Equation C.5 by defining the terms $z_{j}=z-r_{j}$, and constructing two polynomials $G(z)$ and $H(z)$ in terms of $z$,

$$
\begin{aligned}
& G(z)=\sum_{i=0}^{N} G_{j} z^{j}, \\
& H(z)=\sum_{i=0}^{N} H_{j} z^{j} .
\end{aligned}
$$

Using these polynomials, we can rewrite Equation C.5 as,

$$
\bar{w}=\bar{z}-\frac{G}{H} .
$$


The expression for $\kappa$ can be obtained by deriving Equation C.8,

$$
\kappa(z)=\frac{\partial \bar{w}}{\partial z}=\frac{\partial}{\partial z}\left[\frac{G}{H}\right]=\frac{G^{\prime}(z) H(z)-G(z) H^{\prime}(z)}{[H(z)]^{2}} .
$$

The expressions for $G^{\prime}(z)$ and $H^{\prime}(z)$ can be obtained by differentiating Equations C.6 and C.7 to give,

$$
\begin{aligned}
& G^{\prime}(z)=\sum_{j=0}^{N} j G_{j} z^{j-1}=\sum_{j=0}^{N-1} j+1 G_{j+1} z^{j} \\
& H^{\prime}(z)=\sum_{j=0}^{N} j H_{j} z^{j-1}=\sum_{j=0}^{N-1} j+1 H_{j+1} z^{j} .
\end{aligned}
$$

By substituting Equation C.9 into Equation C.3, the critical curve polynomials can be derived as,

$$
\begin{aligned}
\frac{G^{\prime}(z) H(z)-G(z) H^{\prime}(z)}{[H(z)]^{2}} & =e^{i \phi}, \\
G^{\prime}(z) H(z)-G(z) H^{\prime}(z) & =[H(z)]^{2} e^{i \phi}, \\
{\left[G^{\prime}(z) H(z)-G(z) H^{\prime}(z)\right]-[H(z)]^{2} e^{i \phi} } & =0 .
\end{aligned}
$$

Substituting the expressions for $G(z), H(z), G^{\prime}(z)$, and $H^{\prime}(z)$ into Equation C.12, the critical curve polynomial can be obtained as,

$$
\begin{aligned}
{\left[\left(\sum_{k=0}^{N-1}(k+1) G_{k+1} z^{k}\right)\left(\sum_{j=0}^{N} H_{j} z^{j}\right)-\left(\sum_{j=0}^{N} G_{j} z^{j}\right)\left(\sum_{k=0}^{N-1}(k+1) H_{k+1} z^{k}\right)\right] } & \\
-\left[\sum_{j=0}^{N} H_{j} z^{j}\right]^{2} e^{i \phi} & =0, \\
\sum_{j=0}^{N} \sum_{k=0}^{N}\left[\left((k+1) G_{k+1} z^{k}\right)\left(H_{j} z^{j}\right)-\left(G_{j} z^{j}\right)\left((k+1) H_{k+1} z^{k}\right)\left(H_{j} z^{j}\right)\left(H_{k} z^{k}\right)\right] e^{i \phi} & =0 .
\end{aligned}
$$

The polynomial in Equation C.13 has degree of $2 N$, and can be written in the form,

$$
0=\sum_{n=0}^{2 N} c_{k} z^{k}
$$


where the coefficients $c_{n}$ are given as,

$$
c_{k}=\sum_{j=0}^{k}\left[(j+1)\left(G_{j+1} H_{k-j}-G_{k-j} H_{j+1}\right)-H_{j} H_{k-j} e^{i \phi}\right] .
$$

For each value of $\phi$, this polynomial gives $2 N$ roots. These roots correspond to $2 N$ points on the critical curves. Solving the polynomial for a series of $\phi$ values between 0 and $2 \pi$ gives a loci of points on the image plane that describe the critical curves. To obtain the loci of points that describe the caustic curves, these critical curve points can then be inverse-ray traced to the source plane by substituting these points into lens equation.

\section{C.1 Two Lens System - Degree 4 Critical Curve Polynomial}

The lens equation for a two lens system can be expressed as,

$$
w=z-\frac{\epsilon_{1}}{\bar{z}-\bar{r}_{1}}+\frac{\epsilon_{2}}{\bar{z}-\bar{r}_{2}},
$$

where the mass fractions of the planets are represented by the real values $\epsilon_{1}$ and $\epsilon_{2}$ and the 2-dimensional positions of the two lenses are represented by complex positions $r_{1}$ and $r_{2}$ respectively. The complex positions of the critical curve can be obtained by solving the 4th degree polynomial,

$$
0=\sum_{n=0}^{4} c_{n} z^{n}=c_{4} z^{4}+c_{3} z^{3}+c_{2} z^{2}+c_{1} z+c_{0},
$$

where the coefficients of this polynomial can be given by the expressions,

$$
\begin{aligned}
c_{4} & =-H_{2}^{2} e^{i \phi}, \\
c_{3} & =-2 H_{1} H_{2} e^{i \phi}, \\
c_{2} & =\left[G_{2} H_{1}-G_{1} H_{2}\right]-\left[H_{1}^{2}+2 H_{0} H_{2}\right] e^{i \phi}, \\
c_{1} & =2\left[G_{2} H_{0}-G_{0} H_{2}\right]-2 H_{0} H_{1} e^{i \phi}, \\
c_{0} & =\left[G_{1} H_{0}-G_{0} H_{1}\right]-H_{0}^{2} e^{i \phi} .
\end{aligned}
$$


The coefficients of the polynomials $G$ and $H$ are given by,

$$
\begin{aligned}
& H_{2}=1, \\
& H_{1}=r_{1}+r_{2}, \\
& H_{0}=r_{1} r_{2}, \\
& G_{2}=0, \\
& G_{1}=\epsilon_{1}+\epsilon_{2}(=1), \\
& G_{0}=\epsilon_{1} r_{2}+\epsilon_{2} r_{1} .
\end{aligned}
$$

\section{C.2 Three Lens System - Degree 6 Critical Curve Polynomial}

The lens equation for a two lens system can be expressed as,

$$
w=z-\frac{\epsilon_{1}}{\bar{z}-\bar{r}_{1}}+\frac{\epsilon_{2}}{\bar{z}-\bar{r}_{2}}+\frac{\epsilon_{3}}{\bar{z}-\bar{r}_{3}},
$$

where the mass fractions of the planets are represented by the real values $\epsilon_{1}$, $\epsilon_{2}$, and $\epsilon_{3}$, and the 2-dimensional positions of the three lenses are once again represented by complex positions $r_{1}, r_{2}$, and $r_{3}$ respectively. The complex positions

of the critical curve can be obtained by solving the 6th degree polynomial,

$$
0=\sum_{n=0}^{6} c_{n} z^{n}=c_{6} z^{6}+c_{5} z^{5}+c_{4} z^{4}+c_{3} z^{3}+c_{2} z^{2}+c_{1} z+c_{0},
$$

where the coefficients of this polynomial can be given by the expressions,

$$
\begin{aligned}
c_{6} & =-H_{3}^{3} e^{i \phi}, \\
c_{5} & =-2 H_{2} H_{3} e^{i \phi}, \\
c_{4} & =\left[G_{3} H_{2}-G_{2} H_{3}\right]-\left[2 H_{1} H_{3}+H_{2}^{2}\right] e^{i \phi}, \\
c_{3} & =2\left[G_{3} H_{1}-G_{1} H_{3}\right]-\left[2 H_{0} H_{2}+2 H_{1} H_{3}\right] e^{i \phi}, \\
c_{2} & =3\left[G_{3} H_{0}-G_{0} H_{3}\right]+\left[G_{2} H_{1}-G_{1} H_{2}\right]-\left[2 H_{0} H_{2}+H_{1}^{2}\right] e^{i \phi}, \\
c_{1} & =2\left[G_{2} H_{0}-G_{0} H_{2}\right]-2 H_{0} H_{1} e^{i \phi}, \\
c_{0} & =\left[G_{1} H_{0}-G_{0} H_{1}\right]-H_{0}^{2} e^{i \phi} .
\end{aligned}
$$


The coefficients of the polynomials $G$ and $H$ are given by,

$$
\begin{aligned}
& H_{3}=1, \\
& H_{2}=r_{1}+r_{2}+r_{3}, \\
& H_{1}=r_{1} r_{2}+r_{1} r_{3}+r_{2} r_{3}, \\
& H_{0}=r_{1} r_{2} r_{3}, \\
& G_{3}=0, \\
& G_{2}=\epsilon_{1}+\epsilon_{2}+\epsilon_{3}(=1), \\
& G_{1}=\epsilon_{1}\left(r_{2}+r_{3}\right)+\epsilon_{2}\left(r_{1}+r_{3}\right)+\epsilon_{3}\left(r_{1}+r_{2}\right), \\
& G_{0}=\epsilon_{1} r_{2} r_{3}+\epsilon_{2} r_{1} r_{3}+\epsilon_{3} r_{1} r_{2} .
\end{aligned}
$$




\section{Appendix D}

\section{Quadrapole and Hexadecapole Approximations}

While the point source approximation can quickly obtain an estimate for the source magnification, it cannot, by definition, account for the finite size of the source disk or other finite source effects such as limb darkening (see Section 5.1). In contrast, the polygon method discussed in Section 5.3 is designed to include these effects but requires finding the roots for large number of point source positions, which can be very time consuming, especially when limb darkening effects are included. This necessitates a method that can calculate the source magnification quickly while including finite source effects.

The multipole approximations are a class of approximations that can be used to estimate the magnification of the source reasonably quickly, while including finite source effects. The multipole approximations are based on the hexadecapole approximation proposed by Gould (2008). These approximations work by approximating the magnification across the source using the Taylor series. The two multipole approximations of interest are the quadrapole (or 4-pole) and hexadecapole (or 16-pole) approximations, which approximate the magnification up to the second order derivative and the fourth order derivative respectively.

The multipole approximations are classified into two types, the single-point multipole approximations, which calculate the derivatives using a a single point on the source disk, and the multiple-point multipole approximations, which place a number of point source positions at particular locations on the source disk and uses the magnification from each point source position to interpolate the 
magnification across the whole source disk. For reasons explained in Section 5.4.2, the multiple-point multipole approximation is the more accurate type of multipole approximations.

Higher (multiple-point) multipole approximations are also possible but these require a larger number of source points on the source disk. For example, the next two approximations in the sequence, the 64-pole approximation and the 256pole approximation, require 25 source points and 41 source points respectively. In fact, each $4^{n}$-pole approximation requires $2 n(n+1)+1$ source points on the source disk. The number of source positions required for these higher multipole approximations are similar to the number of points required for the polygon method. Since the polygon method is fundamentally better at accounting for finite source effects, it is more efficient in these cases to use the polygon method instead of higher multipole approximations (see Chapter 4).

This appendix discusses how both the single-point and multiple-point multipole approximations can be derived using the Taylor expansion. This derivation mostly focuses on the quadrapole and hexadecapole approximations; however, higher multipole approximations can also be obtained using the derivation described in this appendix.

\section{D.1 Finite Source Disk}

To derive the quadrapole and hexadecapole approximations, we first consider a finite circular source disk of radius $\rho$, and let $A(u, v)$ be the point magnification at any point $(u, v)$ on the source disk. This source disk may have an intensity that varies across the source due to limb darkening, where the intensity of a point on the source disk is dependent on the radial distance from the centre of the source disk.

Suppose $I_{\text {ave }}$ is the average intensity over the disk. The magnification of a finite source is defined as the total apparent brightness of the finite images divided by the brightness of the finite source disk, give as,

$$
A_{\text {finite }}=\frac{\int_{\text {disk }} A(u, v) I(r) d a}{\int_{\text {disk }} I(r) d a}=\frac{\int_{\text {disk }} A(u, v) I(r) d a}{\pi \rho^{2} I_{\text {ave }}} .
$$




\section{D.2 Taylor Expansion}

Consider a function $A(u, v)$ that returns the point magnification for any point $(u, v)$ on the source disk. This function can be represented by a Taylor series, which uses the point source magnification and its derivatives to approximate the magnification anywhere else on the source disk. If $\left(u_{0}, v_{0}\right)$ is the point at the centre of the source disk, and $A\left(u_{0}, v_{0}\right)$ its corresponding magnification, then $A(u, v)$ can be represented by a 2-dimensional Taylor series with $u=u_{0}+\Delta u$ and $v=v_{0}+\Delta v$ as follows,

$$
\begin{aligned}
A\left(u_{0}+\Delta u, v_{0}+\Delta v\right)= & A\left(u_{0}, v_{0}\right) \\
+ & {\left[\frac{\partial A}{\partial u} \Delta u+\frac{\partial A}{\partial v} \Delta v\right] } \\
+ & {\left[\frac{\partial^{2} A}{\partial u^{2}} \frac{(\Delta u)^{2}}{2 !}+\frac{\partial^{2} A}{\partial u \partial v} \Delta u \Delta v+\frac{\partial^{2} A}{\partial v^{2}} \frac{(\Delta v)^{2}}{2 !}\right] } \\
+ & {\left[\frac{\partial^{3} A}{\partial u^{3}} \frac{(\Delta u)^{3}}{3 !}+\frac{\partial^{3} A}{\partial u^{2} \partial v} \frac{(\Delta u)^{2} \Delta v}{2 !}\right.} \\
= & \sum_{n=0}^{\infty}\left[\sum_{k=0}^{n}\left[\frac{\partial^{3} A}{\partial u \partial v^{2}} \frac{\Delta u(\Delta v)^{2}}{2 !}+\frac{\partial^{3} A}{\partial v^{3}} \frac{(\Delta v)^{3}}{3 !} \frac{(\Delta u)^{k}(\Delta v)^{n-k}}{k !(n-k) !}\right]\right]
\end{aligned}
$$

Equation D.2 can then be rewritten as,

$$
\begin{aligned}
A\left(u_{0}+\Delta u, v_{0}+\Delta v\right)= & A_{0,0} \\
& +\left[A_{1,1} \Delta u+A_{1,0} \Delta v\right] \\
& +\left[A_{2,2}(\Delta u)^{2}+A_{2,1} \Delta u \Delta v+A_{2,0}(\Delta v)^{2}\right] \\
& +\left[A_{3,3}(\Delta u)^{3}+A_{3,2}(\Delta u)^{2} \Delta v+A_{3,1} \Delta u(\Delta v)^{2}+A_{3,0}(\Delta v)^{3}\right] \\
= & \sum_{n=0}^{\infty}\left[\sum_{k=0}^{n}\left(A_{n, k}(\Delta u)^{k}(\Delta v)^{n-k}\right)\right],
\end{aligned}
$$


where,

$$
\begin{aligned}
A_{0,0} & =A\left(u_{0}, v_{0}\right) \\
A_{1,1} & =\frac{\partial A\left(u_{0}, v_{0}\right)}{\partial u}, \\
A_{1,0} & =\frac{\partial A\left(u_{0}, v_{0}\right)}{\partial v} \\
A_{2,2} & =\frac{1}{2 !} \frac{\partial^{2} A\left(u_{0}, v_{0}\right)}{\partial u^{2}} \\
A_{2,1} & =\frac{\partial^{2} A\left(u_{0}, v_{0}\right)}{\partial u \partial v}, \\
A_{2,0} & =\frac{1}{2 !} \frac{\partial^{2} A\left(u_{0}, v_{0}\right)}{\partial v^{2}} \\
A_{n, k} & =\frac{1}{k !(n-k) !} \frac{\partial^{n} A\left(u_{0}, v_{0}\right)}{\partial u^{k} \partial v^{n-k}} .
\end{aligned}
$$

\section{D.3 Polar Coordinates}

Since limb darkening depends only on radius, the variation in magnification is radially symmetrical across the source. This symmetry of the source disk can be exploited to make the integral in Equation D.1 easier to solve. Therefore, the point source positions must be converted from Cartesian coordinates into polar coordinates. In doing this, the infinitesimal area becomes $d a=r d \phi d r$, so the Equation D.1 becomes,

$$
\begin{aligned}
A_{\text {finite }} & =\frac{1}{\pi \rho^{2} I_{\text {ave }}} \int_{\text {disk }} A(r, \phi) I(r) d a \\
& =\frac{1}{\pi \rho^{2} I_{\text {ave }}} \int_{0}^{\rho} \int_{0}^{2 \pi} A(r, \phi) I(r) r d \phi d r \\
& =\frac{2}{\rho^{2} I_{\text {ave }}} \int_{0}^{\rho} A(r) I(r) r d r,
\end{aligned}
$$

where,

$$
A(r)=\frac{1}{2 \pi} \int_{0}^{2 \pi} A(r, \phi) d \phi .
$$




\section{D.3.1 Integrating over $\phi$}

The small steps in the $\Delta u$ and $\Delta v$ in Cartesian coordinates can be converted to polar coordinates by the following expressions,

$$
\begin{aligned}
& \Delta u=r \cos \phi, \\
& \Delta v=r \sin \phi .
\end{aligned}
$$

By substituting Equation D.5 and D.6 into Equation D.3, the expression for the magnification becomes,

$$
A(r, \phi)=\sum_{n=0}^{\infty}\left[r^{n} \sum_{k=0}^{n}\left(A_{n, k} \cos ^{n-k} \phi \sin ^{k} \phi\right)\right] .
$$

Therefore, $A(r)$ in Equation D.4 can be then calculated by,

$$
\begin{aligned}
A(r) & =\frac{1}{2 \pi} \int_{0}^{2 \pi} A(r, \phi) d \phi \\
& =\frac{1}{2 \pi} \int_{0}^{2 \pi}\left[\sum_{n=0}^{\infty}\left[r^{n} \sum_{k=0}^{n} A_{n, k} \cos ^{k} \phi \sin ^{n-k} \phi\right]\right] d \phi \\
& =\sum_{n=0}^{\infty}\left[r^{n} \sum_{k=0}^{n}\left(A_{n, k} \frac{1}{2 \pi} \int_{0}^{2 \pi} \cos ^{k} \phi \sin ^{n-k} \phi d \phi\right)\right] .
\end{aligned}
$$

If we consider the odd integers $p$ and $q$, it can be shown that,

$$
\frac{1}{2 \pi} \int_{0}^{2 \pi} \cos ^{p} \phi \sin ^{q} \phi d \phi=0
$$

Therefore, all the odd order derivatives, such as $A_{1,1}, A_{1,0}$, and $A_{2,1}$, are eliminated from Equation D.7. Therefore, without loss of generality, the values of $n$ and $k$ can be transformed by, $n \rightarrow 2 n$ and $k \rightarrow 2 k$. For example, second term in the Taylor series, $n=2$, now corresponds with $n=1$. 
The remaining integrals in Equation D.7 are given by,

$$
\begin{aligned}
\frac{1}{2 \pi} \int_{0}^{2 \pi} \cos ^{2} \phi d \phi & =\frac{1}{2}, \\
\frac{1}{2 \pi} \int_{0}^{2 \pi} \sin ^{2} \phi d \phi & =\frac{1}{2}, \\
\frac{1}{2 \pi} \int_{0}^{2 \pi} \cos ^{4} \phi d \phi & =\frac{3}{8}, \\
\frac{1}{2 \pi} \int_{0}^{2 \pi} \cos ^{2} \phi \sin ^{2} \phi d \phi & =\frac{1}{8}, \\
\frac{1}{2 \pi} \int_{0}^{2 \pi} \sin ^{4} \phi d \phi & =\frac{3}{8} .
\end{aligned}
$$

A general expression for these integrals can be given by the following,

$$
\begin{aligned}
\frac{1}{2 \pi} \int_{0}^{2 \pi} \cos ^{2 p} \phi \sin ^{2 q} \phi d \phi & =\frac{1}{2^{p+q}(p+q) !} \frac{(2 p) !}{2^{p} p !} \frac{(2 q) !}{2^{q} q !} \\
& =\frac{(2 p) !(2 q) !}{2^{2(p+q)}(p+q) ! p ! q !}
\end{aligned}
$$

In terms of $n$ and $k(n=p+q$ and $k=p)$, these integrals can be written as,

$$
\begin{aligned}
\frac{1}{2 \pi} \int_{0}^{2 \pi} \cos ^{2 k} \phi \sin ^{2(n-k)} \phi d \phi & =\frac{1}{2^{n} n !} \frac{(2 k) !}{2^{k} k !} \frac{(2(n-k)) !}{2^{(n-k)}(n-k) !} \\
& =\frac{(2 k) !(2(n-k)) !}{2^{2 n} n ! k !(n-k) !}
\end{aligned}
$$

Therefore, Equation D.7 becomes,

$$
A(r)=\frac{1}{2 \pi} \int_{0}^{2 \pi} A(r, \phi) d \phi=A_{0}+A_{2} r^{2}+A_{4} r^{4}+\cdots=\sum_{n=0}^{\infty} A_{2 n} r^{2 n}
$$


where,

$$
\begin{aligned}
A_{0} & =A_{00} \\
& =A\left(u_{0}, v_{0}\right) \\
A_{2} & =\frac{A_{22}+A_{20}}{2} \\
& =\frac{\frac{1}{2 !} \frac{\partial^{2} A\left(u_{0}, v_{0}\right)}{\partial u^{2}}+\frac{1}{2 !} \frac{\partial^{2} A\left(u_{0}, v_{0}\right)}{\partial v^{2}}}{2} \\
& =\frac{1}{4}\left[\frac{\partial^{2} A\left(u_{0}, v_{0}\right)}{\partial u^{2}}+\frac{\partial^{2} A\left(u_{0}, v_{0}\right)}{\partial v^{2}}\right], \\
A_{4} & =\frac{3 A_{44}+A_{42}+3 A_{40}}{8} \\
& =\frac{\frac{3}{4 !} \frac{\partial^{2} A\left(u_{0}, v_{0}\right)}{\partial u^{4}}+\frac{1}{2 ! 2 !} \frac{\partial^{2} A\left(u_{0}, v_{0}\right)}{\partial u^{2} \partial v^{2}}+\frac{3}{4 !} \frac{\partial^{2} A\left(u_{0}, v_{0}\right)}{\partial v^{4}}}{8} \\
& =\frac{1}{64}\left[\frac{\partial^{2} A\left(u_{0}, v_{0}\right)}{\partial u^{4}}+2 \frac{\partial^{2} A\left(u_{0}, v_{0}\right)}{\partial u^{2} \partial v^{2}}+\frac{3}{4 !} \frac{\partial^{2} A\left(u_{0}, v_{0}\right)}{\partial v^{4}}\right] .
\end{aligned}
$$

A general expression $A_{2 n}$ can be given by,

$$
\begin{aligned}
A_{2 n} & =\sum_{k=0}^{n}\left[\frac{1}{2 \pi} \int_{0}^{2 \pi} \cos ^{k} \phi \sin ^{n-k} \phi d \phi A_{2 n, 2 k}\right] \\
& =\sum_{k=0}^{n}\left[\frac{(2 k) !(2(n-k)) !}{2^{2 n}(n) ! k !(n-k) !} \frac{1}{(2 k) !(2(n-k)) !} \frac{\partial^{2 n} A\left(u_{0}, v_{0}\right)}{\partial u^{2 k} \partial v^{n-k}}\right] \\
& =\sum_{k=0}^{n}\left[\frac{(2 k) !(2(n-k)) !}{2^{2 n}(2 k) !(2(n-k)) !} \frac{1}{n ! k !(n-k) !} \frac{\partial^{2 n} A\left(u_{0}, v_{0}\right)}{\partial u^{2 k} \partial v^{n-k}}\right] \\
& =\sum_{k=0}^{n}\left[\frac{1}{2^{2 n}} \frac{1}{(n !)^{2}} \frac{n !}{k !(n-k) !} \frac{\partial^{2 n} A\left(u_{0}, v_{0}\right)}{\partial u^{2 k} \partial v^{n-k}}\right] \\
& =\frac{1}{2^{2 n}(n !)^{2}} \sum_{k=0}^{n}\left[\left(\begin{array}{l}
n \\
k
\end{array}\right) \frac{\partial^{n} A\left(u_{0}, v_{0}\right)}{\partial u^{k} \partial v^{n-k}}\right] .
\end{aligned}
$$

\section{D.3.2 Integrating over $r$}

After integrating with respect to $\phi$, the expression for the finite magnification becomes,

$$
A_{\text {finite }}=\frac{2}{\rho^{2} I_{\text {ave }}} \int_{r=0}^{\rho} A(r) I(r) r d r=\frac{2}{\rho^{2} I_{\text {ave }}} \int_{r=0}^{\rho}\left(\sum_{n=0}^{\infty} A_{2 n} r^{2 n}\right) I(r) r d r .
$$

However, before we can integrate over $r$, the expression for limb darkening needs to be derived. 


\section{D.4 Limb Darkening}

As discussed in Section 4.2, the observed intensity $I(\psi)$ of a limb darkened star can be approximated by,

$$
I(\psi)=I_{0} \sum_{k=0}^{N} a_{k} \cos ^{k}(\psi)
$$

with $a_{0}=1-u, a_{1}=u, a_{j}=0$ for $j>1$, and $0 \leq u \leq 1$. In this approximation, Equation D.14 can be rewritten as,

$$
\begin{aligned}
& I(\phi)=I_{0}(1-u(1-\cos (\phi))) \\
& I(\phi)=I_{0}\left(1-u\left(1-\sqrt{1-\sin (\phi)^{2}}\right)\right) \\
& I(r)=I_{0}\left(1-u\left(1-\sqrt{1-\left(\frac{r}{a}\right)^{2}}\right)\right),
\end{aligned}
$$

where $r$ is radial distance from centre of source disk, which has radius $\rho$. Since Equation D.13 is written in terms of the average intensity $I_{\text {ave }}$, it is more convenient to rewrite Equation D.15 in terms of $I_{\text {ave }}$, such that,

$$
I(r)=I_{\text {ave }}\left(1-\Gamma\left(1-\frac{3}{2} \sqrt{1-\left(\frac{r}{a}\right)^{2}}\right)\right),
$$

where $\Gamma$ is called the limb darkening coefficient, with $0 \leq \Gamma \leq 1$. This should not be confused with the Gamma Function $\Gamma()$, which appears later in this appendix.

By integrating Equation D.16 across the the source disk, it can be shown that $I_{\text {ave }}$ is indeed the average intensity of the average flux per unit area,

$$
\begin{aligned}
\int I(r) d A & =\int_{0}^{a} I_{a v e}\left(1-\Gamma\left(1-\frac{3}{2} \sqrt{1-\left(\frac{r}{a}\right)^{2}}\right)\right) 2 \pi r d r \\
& =\int_{0}^{a} 2 I_{a v e} \pi(1-\Gamma) r d r+3 I_{a v e} \pi \Gamma \int_{0}^{a} r \sqrt{1-\left(\frac{r}{a}\right)^{2}} d r \\
& =I_{a v e} \pi(1-\Gamma) a^{2}+3 I_{a v e} \pi \Gamma a^{2} \int_{0}^{\pi / 2} \sin \phi \cos ^{2} \phi d \phi \\
& =I_{a v e} \pi a^{2}-I_{a v e} \pi \Gamma a^{2}+3 I_{a v e} \pi \Gamma a^{2} \frac{1}{3} \\
& =I_{\text {ave }} \pi a^{2}-I_{a v e} \pi \Gamma a^{2}+I_{a v e} \pi \Gamma a^{2} \\
& =I_{a v e} \pi a^{2} .
\end{aligned}
$$


The relationship between flux at the centre of the source $I_{0}$ and the average flux per unit area $I_{\text {ave }}$ can be obtained by finding the intensity at $r=0$ using Equation D.16,

$$
I_{0}=I(0)=I_{\text {ave }}\left(1-\Gamma\left(1-\frac{3}{2} \sqrt{1-0}\right)\right)=I_{\text {ave }}\left(1+\frac{\Gamma}{2}\right)
$$

The relationship between $u$ and $\Gamma$ is found by equating the Equations D.15 and D.16.

$$
\begin{aligned}
I_{0}\left(1-u\left(1-\sqrt{1-\left(\frac{r}{a}\right)^{2}}\right)\right) & =I_{\text {ave }}\left(1-\Gamma\left(1-\frac{3}{2} \sqrt{1-\left(\frac{r}{a}\right)^{2}}\right)\right) \\
\left(1+\frac{\Gamma}{2}\right)\left(1-u\left(1-\sqrt{1-\left(\frac{r}{a}\right)^{2}}\right)\right) & =1-\Gamma\left(1-\frac{3}{2} \sqrt{1-\left(\frac{r}{a}\right)^{2}}\right) \\
\frac{\Gamma}{2}-u-u \frac{\Gamma}{2}+\left(u+u \frac{\Gamma}{2}\right) \sqrt{1-\left(\frac{r}{a}\right)^{2}} & =\frac{3 \Gamma}{2} \sqrt{1-\left(\frac{r}{a}\right)^{2}}-\Gamma
\end{aligned}
$$

By collecting the like terms (the terms involving the square root), the relationship between $u$ and $\Gamma$ can be given by,

$$
\begin{aligned}
u \sqrt{1-\left(\frac{r}{a}\right)^{2}+u \frac{\Gamma}{2} \sqrt{1-\left(\frac{r}{a}\right)^{2}}}=\frac{3 \Gamma}{2} \sqrt{1-\left(\frac{r}{a}\right)^{2}} \\
u+u \frac{\Gamma}{2}=\frac{3 \Gamma}{2} \\
\frac{u}{2}(2+\Gamma)=\frac{3 \Gamma}{2} \\
u=\frac{3 \Gamma}{2+\Gamma} .
\end{aligned}
$$

\section{D.4.1 The Integral}

With the expression for limb darkening in Equation D.16, the integrand in Equation D.13 can now be integrated with respect to $r$. Therefore, the expression for 
the total magnification of a finite-source can be derived as follows,

$$
\begin{aligned}
A_{\text {finite }} & =\frac{2}{\rho^{2} I_{\text {ave }}} \int_{0}^{\rho} A(r) r I(r) d r \\
& =\frac{2}{\rho^{2} I_{\text {ave }}}\left(\int_{0}^{\rho} A_{0} r I(r) d r+\int_{0}^{\rho} A_{2} r^{3} I(r) d r+\int_{0}^{\rho} A_{4} r^{5} I(r) d r+\ldots\right) \\
& =\frac{2}{\rho^{2} I_{\text {ave }}} \sum_{0}^{\infty} \int_{0}^{\rho} A_{2 k} r^{2 k+1} I(r) d r \\
& =\frac{2}{\rho^{2}} \sum_{0}^{\infty} \int_{0}^{\rho} A_{2 k} r^{2 k+1}\left(1-\Gamma\left(1-\frac{3}{2} \sqrt{\left.1-\left(\frac{r}{\rho}\right)^{2}\right)}\right) d r\right. \\
& =\frac{2}{\rho^{2}} \sum_{0}^{\infty} \int_{0}^{\rho} A_{2 k} r^{2 k+1} d r-\frac{2 \Gamma}{\rho^{2}} \sum_{0}^{\infty} \int_{0}^{\rho} A_{2 k} r^{2 k+1}\left(1-\frac{3}{2} \sqrt{1-\left(\frac{r}{\rho}\right)^{2}}\right) d r \\
& =\frac{2}{\rho^{2}} \sum_{0}^{\infty} \frac{A_{2 k} \rho^{2 k+2}}{2 k+2}-\frac{2 \Gamma}{\rho^{2}} \sum_{0}^{\infty} A_{2 k} \int_{0}^{\rho} r^{2 k+1} d r \\
& =\sum_{0}^{\infty} \frac{A_{2 k} \rho^{2 k}}{k+1}-\Gamma \sum_{0}^{\infty} \frac{A_{2 k} \rho^{2 k}}{k+1} \sum_{0}^{\infty} A_{2 k}^{\rho} r_{0}^{2 k+1} \sqrt{1-\left(\frac{r}{\rho}\right)^{2}} d r \\
& +\frac{3 \Gamma}{\rho^{2}} \sum_{0}^{\infty} A_{2 k} \int_{0}^{\rho} r^{2 k+1} \sqrt{1-\left(\frac{r}{\rho}\right)^{2}} d r .
\end{aligned}
$$

In Equation D.18 the integral $\int_{0}^{\rho} r^{2 k+1} \sqrt{1-(r / \rho)^{2}} d r$ can be solved by using the equation,

$$
\int_{0}^{L} q^{p} \sqrt{1-\left(\frac{q}{L}\right)^{2}} d q=\frac{\sqrt{\pi} L^{p+1} \Gamma([p+1] / 2)}{4 \Gamma(p / 2+2)} .
$$

Note that $\Gamma()$ in Equation D.19 is the Gamma function, where $\Gamma(k+1)=k$ !, not the limb darkening coefficien. If $p=2 k+1, q=r$, and $L=\rho$ then Equation D.19 becomes,

$$
\int_{0}^{\rho} r^{2 k+1} \sqrt{1-\left(\frac{r}{\rho}\right)^{2}} d r=\frac{\sqrt{\pi} \rho^{2 k+2} \Gamma(k+1)}{4 \Gamma(k+5 / 2)}
$$

with,

$$
\begin{aligned}
\Gamma\left(p+\frac{1}{2}\right) & =\frac{(2 p) ! \sqrt{\pi}}{4^{p} p !} \\
\Gamma\left(k+\frac{5}{2}\right) & =\frac{(2 k+4) ! \sqrt{\pi}}{4^{k+2}(k+2) !} .
\end{aligned}
$$


Using these terms, Equation D.19 becomes,

$$
\begin{aligned}
\int_{0}^{\rho} r^{2 k+1} \sqrt{1-\left(\frac{r}{\rho}\right)^{2}} d r & =\frac{4^{k+2} \sqrt{\pi} \rho^{2 k+2} k !(k+2) !}{4(2 k+4) ! \sqrt{\pi}} \\
& =\frac{4^{k+1} \rho^{2 k+2}(k+1) !(k+2) !}{(k+1)(2 k+4) !}
\end{aligned}
$$

Therefore, Equation D.18 becomes,

$$
\begin{aligned}
A_{\text {finite }}= & \sum_{0}^{\infty} \frac{A_{2 k} \rho^{2 k}}{k+1}-\Gamma \sum_{0}^{\infty} \frac{A_{2 k} \rho^{2 k}}{k+1} \\
& \quad+\frac{3 \Gamma}{\rho^{2}} \sum_{0}^{\infty} A_{2 k} \frac{4^{k+1} \rho^{2 k+2}(k+1) !(k+2) !}{(k+1)(2 k+4) !} \\
= & \sum_{0}^{\infty} \frac{A_{2 k} \rho^{2 k}}{k+1}[1-\Gamma(1-\gamma)],
\end{aligned}
$$

where,

$$
\gamma=3 \frac{4^{k+1}(k+1) !(k+2) !}{(2 k+4) !}
$$

Equation D.22 gives $\gamma=1,4 / 5,24 / 35$ for $k=0,1,2$ respectively. Therefore, the expression for the finite magnification in Equation D.21 can be written as,

$$
\begin{aligned}
A_{\text {finite }} & =\frac{A_{0} \rho^{0}}{1}(1-\Gamma+\Gamma)+\frac{A_{2} \rho^{2}}{2}\left(1-\Gamma+\Gamma \frac{4}{5}\right)+\frac{A_{4} \rho^{4}}{3}\left(1-\Gamma+\Gamma \frac{24}{35}\right)+\cdots . \\
& =A_{0}+\frac{A_{2} \rho^{2}}{2}\left(1-\Gamma \frac{1}{5}\right)+\frac{A_{4} \rho^{4}}{3}\left(1-\Gamma \frac{11}{35}\right)+\cdots
\end{aligned}
$$

The expression for the quadrapole approximation is given by the terms up to and including the second derivative,

$$
A_{\text {quad }}=A_{0}+\frac{A_{2} \rho^{2}}{2}\left(1-\Gamma \frac{1}{5}\right) .
$$

The hexadecapole approximation is given by the terms up to and including the fourth derivative,

$$
A_{\text {hex }}=A_{0}+\frac{A_{2} \rho^{2}}{2}\left(1-\Gamma \frac{1}{5}\right)+\frac{A_{4} \rho^{4}}{3}\left(1-\Gamma \frac{11}{35}\right)
$$




\section{D.5 Calculating the Derivatives}

As mentioned in Section 4.4, the accuracy of a Taylor series depends partly on the accuracy of the derivatives used in the series. Therefore, the accuracy of the estimated finite source magnification will depend on the values for the derivatives $A_{2}$ and $A_{4}$. We investigate two different methods to find these derivatives. The first method is by differentiating the equation for the point source magnification, which we called the single-point multiple approximation method, and the second method is to estimate the derivative with numerical approximations, which we called multiple-point multipole approximation method. The single-point and multiple-point multipole approximations are discussed and derived here.

\section{D.5.1 Single-Point Multiple Approximation}

To obtain expressions for the derivatives of the source amplification, we can convert the derivative in D.10-D.12 from in terms of $u$ and $v$, to in terms of $w$ and $\bar{w}$. To do this, we first need to obtain the first-order partial derivatives for $z, \bar{z}, u$ and $v$,

$$
\begin{array}{ll}
\frac{\partial w}{\partial u}=1 & \frac{\partial w}{\partial v}=i, \\
\frac{\partial \bar{w}}{\partial u}=1 & \frac{\partial \bar{w}}{\partial v}=-i .
\end{array}
$$

Using the chain rule, the second derivative of the source amplification at the point $(u, v)$ can then be given as,

$$
\begin{aligned}
A_{2}= & \frac{A_{u u}+A_{v v}}{4} \\
= & \frac{1}{4}\left[A_{u w} \frac{\partial w}{\partial u}+A_{u \bar{w}} \frac{\partial \bar{w}}{\partial u}+A_{y z} \frac{\partial w}{\partial v}+A_{v \bar{w}} \frac{\partial \bar{w}}{\partial v}\right] \\
= & \frac{1}{4}\left[\left(A_{w w} \frac{\partial w}{\partial u}+A_{w \bar{w}} \frac{\partial \bar{w}}{\partial u}\right) \frac{\partial w}{\partial u}+\left(A_{w w} \frac{\partial w}{\partial u}+A_{w \bar{w}} \frac{\partial \bar{w}}{\partial u}\right) \frac{\partial \bar{w}}{\partial u}\right. \\
& \left.+\left(A_{w w} \frac{\partial w}{\partial v}+A_{w \bar{w}} \frac{\partial \bar{w}}{\partial v}\right) \frac{\partial w}{\partial v}+\left(A_{w w} \frac{\partial w}{\partial v}+A_{w \bar{w}} \frac{\partial \bar{w}}{\partial v}\right) \frac{\partial \bar{w}}{\partial v}\right] \\
= & \frac{1}{4}\left[A_{w w} \frac{\partial w}{\partial u} \frac{\partial w}{\partial u}+2 A_{w \bar{w}} \frac{\partial w}{\partial u} \frac{\partial \bar{w}}{\partial u}+A_{\bar{w} \bar{w}} \frac{\partial \bar{w}}{\partial u} \frac{\partial \bar{w}}{\partial u}\right. \\
& \left.+A_{w w} \frac{\partial w}{\partial v} \frac{\partial w}{\partial v}+2 A_{w \bar{w}} \frac{\partial w}{\partial v} \frac{\partial \bar{w}}{\partial v}+A_{\bar{w} \bar{w}} \frac{\partial \bar{w}}{\partial v} \frac{\partial \bar{w}}{\partial v}\right]
\end{aligned}
$$




$$
\begin{aligned}
A_{2} & =\frac{1}{4}\left[A_{w w}+2 A_{w \bar{w}}+A_{\bar{w} \bar{w}}+A_{w w}(i)(i)+2 A_{w \bar{w}}(i)(-i)+A_{\bar{w} \bar{w}}(-i)(-i)\right] \\
& =\frac{1}{4}\left[A_{w w}+2 A_{w \bar{w}}+A_{\bar{w} \bar{w}}-A_{w w}+2 A_{w \bar{w}}-A_{\bar{w} \bar{w}}\right] \\
& =\frac{1}{4}\left[4 A_{w \bar{w}}\right] \\
& =\frac{\partial^{2} A}{\partial w \partial \bar{w}} .
\end{aligned}
$$

The fourth derivative in Equation D.23 can be expressed as,

$$
\begin{aligned}
A_{4} & =\frac{A_{\text {uиuи }}+2 A_{\text {uuvv }}+A_{v v v v}}{64} \\
& =\frac{1}{4} \frac{\partial^{4} A}{\partial w^{2} \partial \bar{w}^{2}} .
\end{aligned}
$$

The expression for the sixth derivative in Equation D.23 can be given as,

$$
A_{6}=\frac{1}{36} \frac{\partial^{6} A}{\partial w^{3} \partial \bar{w}^{3}}
$$

In fact, these derivatives can be given by the general expression,

$$
A_{2 n}=\frac{1}{(n !)^{2}} \frac{\partial^{2 n} A}{\partial w^{n} \partial \bar{w}^{n}}
$$

The source magnification, $A$, is the sum of the (physical) image magnifications, $\mu$, therefore the source magnification derivatives can be expressed in terms of the image magnification derivatives given in Appendix B, Section B.3, and the Jacobian, $J=\frac{d w d \bar{w}}{d z d \bar{z}}$. Thus the source magnification derivatives can be given as,

$$
\begin{aligned}
A_{2} & =\sum_{\text {images }} \frac{1}{J} \frac{\partial^{2} \mu}{\partial z \partial \bar{z}} \\
A_{4} & =\frac{1}{4} \sum_{\text {images }} \frac{1}{J^{2}} \frac{\partial^{4} \mu}{\partial z^{2} \partial \bar{z}^{2}} \\
A_{6} & =\frac{1}{36} \sum_{\text {images }} \frac{1}{J^{3}} \frac{\partial^{6} \mu}{\partial z^{3} \partial \bar{z}^{3}}, \\
A_{2 n} & =\frac{1}{(n !)^{2}} \sum_{\text {images }} \frac{1}{J^{n}} \frac{\partial^{2 n} \mu}{\partial z^{n} \partial \bar{z}^{n}} .
\end{aligned}
$$




\section{D.5.2 Numerical Approximation of Derivative}

As mentioned in Section 4.4.3, numerical approximations for the derivatives of some function, $f(x)$, can be obtained from the Lagrange polynomials. A Laguerre polynomial is a unique polynomial of degree $k$ that can be interpolated from a set distinct points, $\left(x_{1}, f\left(x_{1}\right)\right), \cdots,\left(x_{k+1}, f\left(x_{k+1}\right)\right)$. The Laguerre polynomial can be differentiated up to the $k^{t h}$ order derivative, and these derivatives describe approximation for the derivatives of the function $f(x)$. Therefore, if we use more distinct points, we can obtain a Laguerre polynomial with a higher degree, and hence the numerical derivatives will be more accurate.

The quadrapole approximation estimates the finite source magnification up to the second order derivative along the $\mathrm{x}$ and $\mathrm{v}$ axes, each requiring 3 points. The centre point of the two second derivatives can be shared between these two derivatives, so the quadrapole approximation requires no less than 5 points.

The hexadecapole approximation estimates the finite source magnification up to the fourth order derivative. The fourth derivative along the $\mathrm{x}$ and $\mathrm{v}$ axes require 5 points each, while 9 points are required for the 'cross derivative' $A_{\text {uuvv }}$. Therefore, the hexadecapole approximation requires no less than 13 points.

\section{Quadrapole Approximation}

In the quadrapole approximation, the second derivative $A_{2}$ can be estimated using the following numerical approximations,

$$
\begin{aligned}
& \frac{\partial^{2} A(u, v)}{\partial u^{2}} \approx \frac{A(u+\rho, v)-2 A(u, v)+A(u-\rho, v)}{\rho^{2}}, \\
& \frac{\partial^{2} A(u, v)}{\partial v^{2}} \approx \frac{A(u, v+\rho)-2 A(u, v)+A(u, v-\rho)}{\rho^{2}} .
\end{aligned}
$$

Therefore, Equation D.11 can be expressed as,

$$
\begin{aligned}
A_{2} \rho^{2} & =\rho^{2} \frac{A_{20}+A_{22}}{2} \\
& =\frac{\rho^{2}}{2}\left[\frac{1}{2 !} \frac{\partial^{2} A(u, v)}{\partial u^{2}}+\frac{1}{2 !} \frac{\partial^{2} A(u, v)}{\partial v^{2}}\right] \\
& =\frac{\rho^{2}}{4}\left[\frac{\partial^{2} A(u, v)}{\partial u^{2}}+\frac{\partial^{2} A(u, v)}{\partial v^{2}}\right]
\end{aligned}
$$




$$
\begin{gathered}
A_{2} \rho^{2} \approx \frac{\rho^{2}}{4}\left[\frac{A(u+\rho, v)-2 A(u, v)+A(u-\rho, v)}{\rho^{2}}\right. \\
\left.+\frac{A(u, v+\rho)-2 A(u, v)+A(u, v-\rho)}{\rho^{2}}\right] \\
\approx \frac{1}{4}[A(u+\rho, v)+A(u-\rho, v)+A(u, v+\rho)+A(u, v-\rho)] \\
-A(u, v) .
\end{gathered}
$$

If we define the term $A_{r,+}$, such that,

$$
A_{r,+}=\frac{1}{4} \sum_{j=0}^{3} A\left(u+r \cos \left(j \frac{\pi}{2}\right), v+r \sin \left(j \frac{\pi}{2}\right)\right)-A_{0}
$$

where $0 \leq r \leq \rho$ is the radial distance from the centre of the source disk, then we can rewrite Equation D.26 as $A_{2} \rho^{2} \approx A_{r,+}$.

Therefore, the magnification of a finite-source disk is approximated by,

$$
A_{\text {finite }} \approx A_{0}+\frac{A_{\rho,+}}{2}\left(1-\Gamma \frac{1}{5}\right)
$$

\section{Hexadecapole Approximation}

In the hexadecapole approximation, the fourth derivative $A_{4}$ can be estimated using the following numerical approximations,

$$
\begin{gathered}
\frac{\partial^{4} A(u, v)}{\partial u^{4}} \approx \frac{16}{\rho^{4}}\left[A(u+\rho, v)-4 A\left(u+\frac{\rho}{2}, v\right)+6 A(u, v)\right. \\
\left.-4 A\left(u-\frac{\rho}{2}, v\right)+A(u-\rho, v)\right] \\
\frac{\partial^{4} A(u, v)}{\partial u^{2} v^{2}} \approx \begin{array}{c}
\frac{16}{\rho^{4}}\left[A\left(u+\frac{\rho}{2}, v+\frac{\rho}{2}\right)+A\left(u+\frac{\rho}{2}, v-\frac{\rho}{2}\right)\right. \\
+A\left(u-\frac{\rho}{2}, v+\frac{\rho}{2}\right)+A\left(u-\frac{\rho}{2}, v-\frac{\rho}{2}\right) \\
-2 A\left(u+\frac{\rho}{2}, v\right)-2 A\left(u-\frac{\rho}{2}, v\right) \\
\left.-2 A\left(u, v+\frac{\rho}{2}\right)-2 A\left(u, v-\frac{\rho}{2}\right)+4 A(u, v)\right]
\end{array} \\
\begin{array}{c}
\frac{\partial^{4} A(u, v)}{\partial v^{4}} \approx \\
\frac{16}{\rho^{4}}\left[A(u, v+\rho)-4 A\left(u, v+\frac{\rho}{2}\right)+6 A(u, v)\right.
\end{array} \\
\left.-4 A\left(u, \frac{\rho}{2}\right)+A(u, v-\rho)\right]
\end{gathered}
$$


Therefore, Equation D.12 can be expressed as,

$$
\begin{aligned}
A_{4} \rho^{4} & =\rho^{4} \frac{3 A_{40}+A_{42}+3 A_{44}}{8} \\
& =\rho^{4} \frac{\frac{3}{4 !} \frac{\partial^{4} A}{\partial u^{4}}+\frac{1}{2 ! 2 !} \frac{\partial^{4} A}{\partial u^{2} \partial v^{2}}+\frac{3}{4 !} \frac{\partial^{4} A}{\partial v^{4}}}{8} \\
A_{4} \rho^{4} & \approx 2 A_{\frac{\rho}{\sqrt{2}}, \times}+A_{\rho,+}-8 A_{\frac{\rho}{2},+},
\end{aligned}
$$

where,

$$
A_{r, \times}=\frac{1}{4} \sum_{j=0}^{3} A\left(u+r \cos \left(\frac{\pi}{4}+j \frac{\pi}{2}\right), v+r \sin \left(\frac{\pi}{4}+j \frac{\pi}{2}\right)\right)-A_{0} .
$$

Since the fourth derivative requires five points along the $u$ and $v$ axes, the second derivatives can be also be estimated using the same five points for each axis. This gives a much better approximation for the second derivative than the quadrapole approximation which estimates the second derivative using only 3 points along each axis. Therefore, the second derivative term $A_{2}$ can be approximated as,

$$
\begin{array}{r}
\frac{\partial^{2} A(u, v)}{\partial u^{2}} \approx \frac{-1}{3 \rho^{2}}\left[A(u+\rho, v)-16 A\left(u+\frac{\rho}{2}, v\right)+30 A(u, v)\right. \\
\left.-16 A\left(u-\frac{\rho}{2}, v\right)+A(u-\rho, v)\right], \\
\frac{\partial^{2} A(u, v)}{\partial v^{2}} \approx \frac{-1}{3 \rho^{2}}\left[A(u, v+\rho)-16 A\left(u, v+\frac{\rho}{2}\right)+30 A(u, v)\right. \\
\left.-16 A\left(u, v-\frac{\rho}{2}\right)+A(u, v-\rho)\right] .
\end{array}
$$

Using these terms, Equation D.11 can be expressed as,

$$
\begin{aligned}
A_{2} \rho^{2}= & \rho^{2} \frac{A_{20}+A_{22}}{2} \\
= & \frac{\rho^{2}}{2}\left[\frac{1}{2 !} \frac{\partial^{2} A(u, v)}{\partial u^{2}}+\frac{1}{2 !} \frac{\partial^{2} A(u, v)}{\partial v^{2}}\right] \\
= & \frac{\rho^{2}}{4}\left[\frac{\partial^{2} A(u, v)}{\partial u^{2}}+\frac{\partial^{2} A(u, v)}{\partial v^{2}}\right] \\
= & \frac{\rho^{2}}{4}\left[\frac { - 1 } { 3 \rho ^ { 2 } } \left[A(u+\rho, v)-16 A\left(u+\frac{\rho}{2}, v\right)+30 A(u, v)\right.\right. \\
& \quad+\frac{-1}{3 \rho^{2}}\left[A(u, v+\rho)-16 A\left(u, v+\frac{\rho}{2}\right)+30 A(u, v)\right. \\
A_{2} \rho^{2} \approx & \frac{1}{3}\left[16 A_{\frac{\rho}{2},+}-A_{\rho,+}\right] .
\end{aligned}
$$


Therefore, the hexadecapole approximation approximates the magnification of a finite-source disk as,

$$
A_{\text {finite }} \approx A_{0}+\frac{16 A_{\frac{\rho}{2},+}-A_{\rho,+}}{6}\left(1-\Gamma \frac{1}{5}\right)+\frac{2 A_{\frac{\rho}{\sqrt{2}}, \times}+A_{\rho,+}-8 A_{\frac{\rho}{2},+}}{3}\left(1-\Gamma \frac{11}{35}\right)
$$




\section{Appendix E}

\section{Laguerre and Jenkins-Traub Algorithms}

The lens equation for a multiple lens system described in Chapter 2 cannot be solved analytically. Instead, these polynomials need to be solved numerically, via polynomial root finder algorithms. The two particular algorithms of interest in this thesis were the Jenkins-Traub algorithm and the Laguerre algorithm. These algorithms were briefly described in Chapter 5 .

This appendix aims to discuss the details of the structure and processes of these two algorithms, including the equations these algorithms use to find these roots. This appendix does not cover the proof of convergence nor the rate of convergence for these algorithms, but this can be found in Jenkins and Traub (1970) and Wilkinson (1965) for the Jenkins-Traub and Laguerre algorithms respectively.

\section{E.1 Laguerre Algorithm}

The polynomial $P(z)=0$ can be represented in two ways: either as a sum of the coefficients $c_{k}$, or as a product of its roots $\zeta_{i}$,

$$
\begin{aligned}
& 0=\sum_{k=0}^{n} c_{k} z^{k}, \\
& 0=c_{n} \prod_{i=1}^{n}\left(z-\zeta_{i}\right),
\end{aligned}
$$

where $C=c_{n}$ is the coefficient of $z^{n}$ in this polynomial. From Equation E.2, 
it can be shown that the first and second derivatives can be given by,

$$
\begin{aligned}
\frac{P^{\prime}(z)}{P(z)} & =\frac{d}{d z} \ln |P(z)|=\sum_{i=1}^{n} \frac{1}{z-\zeta_{i}} \\
\frac{P^{\prime}(z)^{2}-P^{\prime \prime}(z) P(z)}{P(z)^{2}} & =\frac{d^{2}}{d z^{2}} \ln |P(z)|=\sum_{i=1}^{n} \frac{1}{\left(z-\zeta_{i}\right)^{2}} .
\end{aligned}
$$

If we assume that there is one root $\zeta_{1}$ at a close distance $a$ from the initial value $z_{0}$, and the roots $\zeta_{1}, \cdots, \zeta_{n}$ are all at a distance $b$ from this value, Equations E.3 and E.4 can respectively be expressed as,

$$
\begin{aligned}
\frac{P^{\prime}(z)}{P(z)} & =G=\frac{1}{a}+\frac{n-1}{b}, \\
\frac{P^{\prime}(z)^{2}-P^{\prime \prime}(z) P(z)}{P(z)^{2}} & =H=\frac{1}{a^{2}}+\frac{n-1}{b^{2}} .
\end{aligned}
$$

Equations E.5 and E.6 can then be combined, eliminating $b$ to give a value for $a$ as,

$$
a=\frac{n}{G \pm \sqrt{(n-1)\left(n H-G^{2}\right)}} .
$$

In order to prevent the algorithm overshooting the root, the sign of the square root is chosen to give a larger denominator, giving a smaller value for $a$. Once $a$ is calculated, the next starting position $z$ is calculated as $z-a \rightarrow z$. This process repeats until $a$ is smaller than the machine precision or $P(z)$ is smaller than the acceptable numerical error, implying that $z \approx \zeta_{1}$ to within its numerical precision. Then the algorithm repeats the process to find the next root, $\zeta_{2}$. The limit cycle breaking technique is implemented in zroots to break non-converging infinite loops of $z$ values, in the rare cases when these loops form. zroots also uses deflation and polishing to improve the accuracy and efficiency of the algorithm. Deflation, polishing and limit cycle breaking are discussed in Chapter 5 .

\section{E.2 Jenkins-Traub Algorithm}

The Jenkins-Traub algorithm is a three staged algorithm developed Jenkins \& Traub (1970). This algorithm is described here as it is appears in the FORTRAN 
subroutine CPOLY (Jenkins \& Traub 1972). In a similar style to Laguerre's Method, the roots of a polynomial are found in roughly increasing order to reduce the numerical errors caused by deflating the polynomial by a large root. This algorithm also employs deflation and breaking limit cycles.

The Jenkins-Traub algorithm works by generating a sequence of polynomials $H^{(\lambda)}(z)$ such that $H^{(\lambda)}(z) \rightarrow P_{1}(z)$ as $\lambda \rightarrow \infty$ where $P_{i}(z)$ are the Laguerre factors, or the co-factors of the roots $\zeta_{i}$, given by,

$$
P_{i}(z)=\frac{P(z)}{z-\zeta_{i}}
$$

From Equation E.3, it can be shown that,

$$
P^{\prime}(z)=\sum_{i=1}^{k} m_{i} P_{i}(z),
$$

where $m_{i}$ is the multiplicity of the $i$ th root and $k$ is number of roots. We can then generate a sequence of polynomials $H^{(\lambda)}(z)$ with $H^{(0)}(z)=P^{\prime}(z)$, such that,

$$
H^{(\lambda)}(z)=\sum_{i=1}^{k} d_{i}^{(\lambda)} P_{i}(z)
$$

where $d_{i}^{(0)}=m_{i}$ for $i=1,2, \ldots k$. We can then choose the sequence such that $d_{j}^{(\lambda)} / d_{1}^{(\lambda)} \rightarrow 0$ as $\lambda \rightarrow \infty$. In this limit, Equation E.10 becomes,

$$
\lim _{\lambda \rightarrow \infty} H^{(\lambda)}(z)=P_{1}(z)=\frac{P(z)}{z-\zeta_{1}} .
$$

Therefore, when $\lambda$ is large, the value for the $\operatorname{root} \zeta_{i}$ can be approximated from $H^{(\lambda)}(z)$. For each value of $\lambda$, we can calculate the next polynomial $H^{(\lambda+1)}(z)$ using the polynomials $P(z)$ and $H^{(\lambda)}(z)$. All three stages in this algorithm calculates $H^{(\lambda+1)}(z)$ using to the equation,

$$
\bar{H}^{(\lambda+1)}(z)=\frac{1}{z-s_{\lambda}}\left[\frac{P(s)}{\bar{H}^{(\lambda)}\left(s_{\lambda}\right)} \bar{H}^{(\lambda)}(z)-P(z)\right],
$$

where $\bar{H}^{(\lambda)}(z)$ represents the normalised form of $H^{(\lambda)}(z)$ (i.e. the leading coefficient set to 1$)$. However, the value for $s_{\lambda}$ differs for each stage. The value 
for $s_{\lambda}$ represents the origin of this equation and shifting this value closer to the root attempts to make $\bar{H}^{(\lambda)}(z)$ converge faster to $P_{i}(z)$.

The first stage of this algorithm is called the No Shift stage, which uses $s_{\lambda}=0$. The second stage is called the Fixed Shift stage, in which the origin is shifted by a fixed value $s_{\lambda}=s$ throughout this stage. The third and final stage is called the Variable Shift stage, which calculates a new value for $s_{\lambda}$ using the polynomial $H^{(\lambda)}(z)$, in attempt to move the origin closer to the root after each iteration.

\section{E.2.1 Stage One: No shift}

The polynomial $\bar{H}^{(\lambda+1)}(z)$ is calculated by the equation,

$$
\bar{H}^{(\lambda+1)}(z)=\frac{1}{z}\left[\frac{P(0)}{\bar{H}^{(\lambda)}(0)} \bar{H}^{(\lambda)}(z)-P(z)\right] .
$$

It can be shown that Equation E.13 is equivalent to,

$$
\bar{H}^{(\lambda+1)}(z)=\sum_{i=1}^{k} \frac{m_{i}}{\zeta_{i}^{\lambda}} P_{i}(z),
$$

for $\lambda=0, \ldots, M-1$. Clearly, if $\zeta_{1}<\zeta_{i}$ for $i=2, \ldots, k$, then $\frac{m_{1}}{m_{i}} \frac{\zeta_{i}}{\zeta_{1}} \rightarrow 0$ as $\lambda \rightarrow \infty$. Although the sequence $H^{(\lambda)}(z)$ will eventually converge to $P_{i}(z)$, it may take many iterations until $\bar{H}^{(\lambda)}(z) \approx P_{i}(z)$ to within the numerical precision. Therefore, stages two and three attempt to shift the origin closer to the root so that $\zeta_{i}$ is relatively smaller, and $\bar{H}^{(\lambda)}(z)$ converges faster to $P_{i}(z)$.

Stage one is terminated and stage two is started after 5 iterations (i.e. $M=5$ ), as determined by numerical experience (Jenkins \& Traub 1970). Although stage one is not necessary from a theoretical perspective, it is useful for accentuating the smaller roots.

\section{E.2.2 Stage Two: Fixed shift}

Stage two attempts to effectively shift the origin closer to the root by a fixed value $s$, where $|s|=\beta$. $\beta$ is given by the lower bound for the modulus of the roots, which can be found from the coefficients $c_{j}$ of polynomial $P(z)$,

$$
y=\left|c_{N}\right| x^{N}+\left|c_{N-1}\right| x^{N-1}+\cdots+\left|c_{1}\right| x-\left|c_{0}\right|
$$


At $x=0$, the value of polynomial given in Equation E.15 is $y=-\left|c_{0}\right|<0$. However, for all $x>0$, the first, second, and all higher derivatives are nonnegative, so in this range, the polynomial is monotonically increasing. Therefore, the polynomial has only one positive $\operatorname{root} \beta$, which can easily be found using the Newton-Raphson iteration. A random phase is then assigned to $\beta$ to give the complex value $s$.

For $\lambda=M, M+1, \cdots, L-1$ The polynomial $\bar{H}^{(\lambda+1)}(z)$ is calculated by the equation,

$$
\bar{H}^{(\lambda+1)}(z)=\frac{1}{z-s}\left[\frac{P(s)}{\bar{H}^{(\lambda)}(s)} \bar{H}^{(\lambda)}(z)-P(z)\right] .
$$

It can be shown that the combination of Equations E.13 and E.16 is equivalent to,

$$
\bar{H}^{(\lambda+1)}(z)=\sum_{i=1}^{k} \frac{m_{i}}{\zeta_{i}^{M}\left(\zeta_{i}-s\right)^{\lambda-M}} P_{i}(z) .
$$

From Equation E.17, it can clearly be seen that the $\bar{H}^{(\lambda+1)}(z)$ sequence will quickly converge to the root $\zeta_{1}$ where $\left|\zeta_{1}-s\right|<\left|\zeta_{i}-s\right|$ where $s=2,3, . . k$. The main purpose of the second stage is to separate equimodular or almost equimodular roots.

Stage two is terminated and stage three starts at iteration $L$, when so called weak convergence is satisfied in two successive iterations,

$$
\left|t_{L}-t_{L-1}\right| \leq \frac{1}{2}\left|t_{L-1}\right| \quad \text { and } \quad\left|t_{L-1}-t_{L-2}\right| \leq \frac{1}{2}\left|t_{L-2}\right| .
$$

where,

$$
t_{\lambda}=s-\frac{P(z)}{\bar{H}^{(\lambda)}(z)}
$$

\section{E.2.3 Stage Three: Variable Shift}

Stage three attempts to effectively shift the origin closer to the root by $s_{\lambda}$, a value which changes at each iteration. For the first iteration in this stage, the 
shift is determined by,

$$
s_{L}=s-\frac{P(s)}{\bar{H}^{L}(s)} .
$$

For each iteration the polynomial $\bar{H}^{(\lambda+1)}(z)$ is calculated by the equation,

$$
\bar{H}^{(\lambda+1)}(z)=\frac{1}{z-s_{\lambda}}\left[\frac{P\left(s_{\lambda}\right)}{\bar{H}^{(\lambda)}\left(s_{\lambda}\right)} \bar{H}^{(\lambda)}(z)-P(z)\right] .
$$

It can be shown that, when combined with stages one and two, this equation (above) is equivalent to,

$$
H^{(\lambda+1)}(z)=\sum_{i=1}^{k} \frac{m_{i}}{\zeta_{i}^{M}\left(\zeta_{i}-s\right)^{\lambda-M} \prod_{\lambda=L}\left(\zeta_{i}-s_{\lambda}\right)} P_{i}(z) .
$$

The shift is then determined by,

$$
s_{\lambda+1}=s_{\lambda}-\frac{P\left(s_{\lambda}\right)}{\bar{H}^{\lambda+1}\left(s_{\lambda}\right)} .
$$

Equation E.23 is identical to the Newton-Raphson iteration $x_{i+1}=x_{i}-\frac{f(x)}{f^{\prime}(x)}$. As the polynomial sequence $H^{\lambda}(z)$ converges to $P_{1}(z), s_{\lambda+1}$ gets closer to the $\operatorname{root} \zeta_{1}$.

When $P\left(s_{\lambda}\right)$ is smaller than the acceptable numerical error, stage three is terminated. The root is then stored and the polynomial is deflated, and the algorithm starts finding the next root. 


\section{Appendix F}

\section{Control Files}

The control files define the parameter values for specific microlensing models, which are used by mlens2 to calculate the model lightcurve and $\chi^{2}$ value. Each control file is written as a plain text file and has the file extension .ctl. Each control file begins with the line \#CTLFILEV3 and is followed by lines specifying the values for the parameters, usually grouped into six sections,

- \#CTLFILEV3: Indicates the beginning of the control file. The lines that follow usually contain non-model parameters, such as the event name and the celestial coordinates for the lens.

- PARAMETERS: This section contains the values for the model parameters.

- INTERACTIVE: This section contains the parameters for producing the critical curves, caustic curves and the magnification maps.

- LIGHTCURVE: This section contains the parameters for producing the lightcurves, including the number of source positions that make up the lightcurve.

- PASSBANDS: This section contains the (linear) limb darkening parameters for each range of wavelength in the observational data.

- OBSERVATIONS: This section contains the list of the observational data files and the passband required for each data file. 
The model parameters for the lens mass are defined in terms of mass ratios relative to the primary lens, which by definition has a mass ratio of 1 . The mlens 2 code internally converts these mass ratios into mass fractions (where the sum of the mass fractions gives 1). The parameters for time are given in units of Heliocentric Julian Days - 2450000.

The parameters for separations and lengths are expressed in units of Einstein radii with total mass defined as 1 , and all angles are in degrees. The lens separations are measured from the primary lens, and these are angles measured from the horizontal axis on lens plane. When these lenses are positioned on the lens plane, the centre of mass is positioned at the origin, and the source parameters are measured from this centre of mass. The impact parameter is defined so that a positive impact parameter with an angle of zero and no orbital motion gives a horizontal source track going from left to right above centre of mass, and a negative impact parameter gives this horizontal source track going from left to right below the centre of mass. Increasing the impact angle rotates this source track in the anti-clockwise direction about the centre of mass, and decreasing the impact angle rotates it in the clockwise direction.

Some examples of control files used to generate the lightcurves in Chapter 6 are shown here. 


\begin{tabular}{|c|c|c|}
\hline \multicolumn{3}{|l|}{ \#CTLFILEV3 } \\
\hline EventName & \multicolumn{2}{|l|}{ 09moa-319 } \\
\hline LimbDarken & \multicolumn{2}{|l|}{ true } \\
\hline MinVertexCount & \multicolumn{2}{|l|}{50} \\
\hline LimbRings & \multicolumn{2}{|l|}{5} \\
\hline MaxVertexSeparation & \multicolumn{2}{|c|}{0.001} \\
\hline RightAscention & \multicolumn{2}{|c|}{$1806 \quad 58.13$} \\
\hline Declination & \multicolumn{2}{|c|}{$-26 \quad 49 \quad 10.89$} \\
\hline PerihelionTime & \multicolumn{2}{|c|}{4834.80} \\
\hline EquinoxTime & \multicolumn{2}{|l|}{4910.99} \\
\hline \multicolumn{3}{|l|}{ PARAMETERS } \\
\hline ImpactParameter & \multicolumn{2}{|l|}{-0.0062} \\
\hline ImpactAngle & \multicolumn{2}{|l|}{330.4649} \\
\hline SourceRadius & \multicolumn{2}{|l|}{0.001929} \\
\hline Companion1Angle & \multicolumn{2}{|l|}{0} \\
\hline Companion1Distance & \multicolumn{2}{|l|}{0.97537} \\
\hline Companion1Mass & \multicolumn{2}{|l|}{$3.95 \mathrm{E}-4$} \\
\hline PeakTime & \multicolumn{2}{|l|}{5006.99482} \\
\hline CrossingTime & 16.57 & \\
\hline StartTime & 5004 & \\
\hline EndTime & 5009 & \\
\hline INTERACTIVE & & \\
\hline Viewport & $-0.12 \quad 0.12$ & $2-0.12 \quad 0.12$ \\
\hline Steps & 500 & \\
\hline LIGHTCURVE & & \\
\hline UseObservations & true & \\
\hline UniformSteps & 1000 & \\
\hline UniformPassband & 3 & \\
\hline PASSBANDS & & \\
\hline \# V band & & \\
\hline 10.6630 & & \\
\hline \# $\mathrm{R}$ band & & \\
\hline 20.5887 & & \\
\hline \# I band & & \\
\hline 30.5090 & & \\
\hline \# $\mathrm{H}$ band & & \\
\hline 40.3292 & & \\
\hline \# Farm Cove, unfilte & red & \\
\hline 50.5413 & & \\
\hline \# Auck, unfiltered & & \\
\hline 60.5490 & & \\
\hline OBSERVATIONS & & \\
\hline phot_auck_mb09319_co & nv_norm & 2 \\
\hline phot_bci_mb09310_con & v_norm & 3 \\
\hline phot_bcv_mb09310_con & V_norm & 1 \\
\hline phot_bron_mb09310_co & nv_norm & \\
\hline phot_cao_mb09310_con & v_norm & \\
\hline phot_ctioi_mb09310_c & onv_norm & 3 \\
\hline phot_danish_mb09310_ & conv_norm & 3 \\
\hline phot_fco_mb09310_con & v_norm & 5 \\
\hline phot_ftn_mb09310_con & iv_norm & \\
\hline phot_fts_mb09310_con & iv_norm & 2 \\
\hline phot_iac_mb09310_con & v_norm & 3 \\
\hline phot_loao_mb09310_co & nv_norm & \\
\hline phot_lt_mb09310_conv & _norm & 3 \\
\hline phot_moa_mb09310_con & v_norm & 2 \\
\hline phot_pal_mb09310_con & v_norm & 3 \\
\hline phot_sso_mb09310_con & v_norm & \\
\hline phot_vlo_mb09310_con & iv_norm & \\
\hline phot_wC_mb09310_conv & _norm & \\
\hline
\end{tabular}

Figure F.1: Control file used to model MOA-2009-BLG-319 and used to generate the lightcurves in Figures 6.1 and 6.3. 


\begin{tabular}{|c|c|}
\hline \multicolumn{2}{|l|}{ \#CTLFILEV3 } \\
\hline EventName & $06 o b-109$ \\
\hline MinVertexCount & 50 \\
\hline LimbRings & 5 \\
\hline LimbRingsSpaceRadius & true \\
\hline MaxVertexSeparation & 0.001 \\
\hline RightAscention & $17 \quad 52 \quad 34.51$ \\
\hline Declination & $\begin{array}{lll}-30 & 05 & 16.0\end{array}$ \\
\hline PerihelionTime & 3737.12 \\
\hline EquinoxTime & 3815.27 \\
\hline \multicolumn{2}{|l|}{ PARAMETERS } \\
\hline ImpactParameter & 0.003479 \\
\hline ImpactAngle & 144.5555328 \\
\hline SourceRadius & 0.0003120188531 \\
\hline Companion1Angle & 180.0112307 \\
\hline Companion1Distance & 0.6263753290 \\
\hline Companion1Mass & 0.001358729084 \\
\hline Companion2Angle & -13.49888565 \\
\hline Companion2Distance & 1.04185 \\
\hline Companion20rbit & 0.001690 .00181 \\
\hline Companion20rbitPeriod & 4901.960784 \\
\hline Companion2Mass & 0.0005061020381 \\
\hline PeakTime & 3831.0197 \\
\hline CrossingTime & 127.300 \\
\hline StartTime & 3820 \\
\hline EndTime & 3840 \\
\hline ParallaxScale & 0.3620 \\
\hline ParallaxAngle & 156.3945597 \\
\hline INTERACTIVE & \\
\hline Viewport & $\begin{array}{lllll}-0.06 & 0.17 & -0.06 & 0.02\end{array}$ \\
\hline Steps & 500 \\
\hline LIGHTCURVE & \\
\hline UseObservations & true \\
\hline UniformSteps & 1000 \\
\hline UniformPassband & 3 \\
\hline PASSBANDS & \\
\hline \# V band & \\
\hline 10.6630 & \\
\hline$\# \mathrm{R}$ band & \\
\hline 20.5887 & \\
\hline \# I band & \\
\hline 30.5090 & \\
\hline$\# \mathrm{H}$ band & \\
\hline 40.3292 & \\
\hline \# Farm Cove, unfiltere & \\
\hline 50.5413 & \\
\hline \# Auck, unfiltered & \\
\hline 60.5490 & \\
\hline OBSERVATIONS & \\
\hline 06ob-109-auck & \\
\hline $06 o b-109-f c o v$ & \\
\hline 06ob-109-liv & \\
\hline 06ob-109-mdm & \\
\hline 06ob-109-mfun & \\
\hline 06ob-109-moa & \\
\hline 06ob-109-mtlem & \\
\hline 06ob-109-ogle & \\
\hline $06 o b-109-\operatorname{tas}$ & \\
\hline 06ob-109-wise & \\
\hline
\end{tabular}

Figure F.2: Control file used to model OGLE-2006-BLG-109 and used to generate the lightcurves in Figures 6.4 and 6.6. 


\begin{tabular}{|c|c|c|c|c|c|c|}
\hline \multicolumn{7}{|l|}{ \#CTLFILEV3 } \\
\hline \multicolumn{2}{|l|}{ EventName } & \multicolumn{5}{|l|}{ 06ob-109 } \\
\hline \multicolumn{2}{|c|}{ MaxVertexSeparation } & \multicolumn{5}{|l|}{0.001} \\
\hline \multicolumn{2}{|c|}{ MinVertexCount } & \multicolumn{5}{|l|}{50} \\
\hline \multicolumn{2}{|l|}{ LimbRings } & \multicolumn{5}{|l|}{5} \\
\hline \multicolumn{2}{|c|}{ LimbRingsSpaceRadius } & \multicolumn{5}{|l|}{ true } \\
\hline \multicolumn{2}{|c|}{ RightAscention } & \multicolumn{5}{|l|}{$17 \quad 52 \quad 34.51$} \\
\hline \multicolumn{2}{|l|}{ Declination } & -300516 & & & & \\
\hline PerihelionTime & & 3737.12 & & & & \\
\hline EquinoxTime & & 3815.27 & & & & \\
\hline PARAMETERS & & & & & & \\
\hline ImpactParameter & & 0.003479 & 0.003 & 3514 & 3 & \\
\hline ImpactAngle & & 144.5555328 & 144.6 & & 3 & \\
\hline SourceRadius & & 0.0003120188531 & 0.000 & 038 & 3 & \\
\hline ParallaxScale & & 0.362 & & & & \\
\hline ParallaxAngle & & 156.3945597 & & & & \\
\hline Companion1Angle & & 180.0112307 & & & & \\
\hline Companion1Distan & nce & 0.626375329 & & & & \\
\hline Companion1Mass & & 0.001358729084 & & & & \\
\hline Companion2Angle & & -13.49888565 & & & & \\
\hline Companion2Distan & nce & 1.04185 & & & & \\
\hline Companion20rbit & & 0.001690 .00181 & & & & \\
\hline Companion20rbitP & Period & 4901.960784 & & & & \\
\hline Companion2Mass & & 0.0005061020381 & & & & \\
\hline PeakTime & & 3831.0197 & 3831 . & .03 & 3 & \\
\hline CrossingTime & & 127.3 & 128 & & 3 & \\
\hline StartTime & & 3820 & & & & \\
\hline EndTime & & 3840 & & & & \\
\hline INTERACTIVE & & & & & & \\
\hline Viewport & & $\begin{array}{lll}-0.06 & 0.17 & -0.06\end{array}$ & 0.032 & & & \\
\hline Steps & & 500 & & & & \\
\hline LIGHTCURVE & & & & & & \\
\hline UseObservations & & true & & & & \\
\hline UniformSteps & & 1000 & & & & \\
\hline UniformPassband & & 3 & & & & \\
\hline PASSBANDS & & & & & & \\
\hline \# V band & & & & & & \\
\hline 10.6630 & & & & & & \\
\hline$\# \mathrm{R}$ band & & & & & & \\
\hline 20.5887 & & & & & & \\
\hline \# I band & & & & & & \\
\hline 30.5090 & & & & & & \\
\hline$\# \mathrm{H}$ band & & & & & & \\
\hline 40.3292 & & & & & & \\
\hline \# Farm Cove, unf & filtered & & & & & \\
\hline 50.5413 & & & & & & \\
\hline \# Auck, unfilter & red & & & & & \\
\hline 60.5490 & & & & & & \\
\hline OBSERVATIONS & & & & & & \\
\hline 06ob-109-auck & 6 & & & & & \\
\hline 06ob-109-fcov & 5 & & & & & \\
\hline 06ob-109-liv & 2 & & & & & \\
\hline 06ob-109-mdm & 3 & & & & & \\
\hline 06ob-109-mfun & 3 & & & & & \\
\hline 06ob-109-moa & 3 & & & & & \\
\hline 06ob-109-mtlem & 3 & & & & & \\
\hline 06ob-109-ogle & 3 & & & & & \\
\hline $06 o b-109-$ tas & 3 & & & & & \\
\hline 06ob-109-wise & 2 & & & & & \\
\hline RESULTS & & & & & & \\
\hline ImpactParameter & Impact $\mathrm{A}$ & ngle SourceRadius & PeakTime & Crossin & gTime & ChiSquare \\
\hline 0.0034965 & 144.556 & 0.000346009 & 3831.02 & 127.3 & & 1799.72 \\
\hline 0.003479 & 144.556 & 0.000346009 & 3831.02 & 127.3 & & 1842.91 \\
\hline 0.003479 & 144.556 & 0.000346009 & 3831.02 & 127.65 & & 1901.7 \\
\hline
\end{tabular}

Figure F.3: Parameters used to do a small grid search to find a better model for OGLE-2006BLG-109. The results of the three models with the smallest $\chi^{2}$ value are also shown in this file. 


\begin{tabular}{|c|c|}
\hline \multicolumn{2}{|l|}{ \#CTLFILEV3 } \\
\hline EventName & $06 o b-109$ \\
\hline MinVertexCount & 50 \\
\hline LimbRings & 5 \\
\hline LimbRingsSpaceRadius & true \\
\hline MaxVertexSeparation & 0.001 \\
\hline RightAscention & $1752 \quad 34.51$ \\
\hline Declination & -300516.0 \\
\hline PerihelionTime & 3737.12 \\
\hline EquinoxTime & 3815.27 \\
\hline \multicolumn{2}{|l|}{ PARAMETERS } \\
\hline ImpactParameter & 0.0034965 \\
\hline ImpactAngle & 144.556 \\
\hline SourceRadius & 0.000346009 \\
\hline Companion1Angle & 180.0112307 \\
\hline Companion1Distance & 0.6263753290 \\
\hline Companion1Mass & 0.001358729084 \\
\hline Companion2Angle & -13.49888565 \\
\hline Companion2Distance & 1.04185 \\
\hline Companion20rbit & 0.001690 .00181 \\
\hline Companion20rbitPeriod & 4901.960784 \\
\hline Companion2Mass & 0.0005061020381 \\
\hline PeakTime & 3831.02 \\
\hline CrossingTime & 127.3 \\
\hline StartTime & 3820 \\
\hline EndTime & 3840 \\
\hline ParallaxScale & 0.3620 \\
\hline ParallaxAngle & 156.3945597 \\
\hline INTERACTIVE & \\
\hline Viewport & $\begin{array}{lllll}-0.06 & 0.17 & -0.06 & 0.032\end{array}$ \\
\hline Steps & 500 \\
\hline LIGHTCURVE & \\
\hline UseObservations & true \\
\hline UniformSteps & 1000 \\
\hline UniformPassband & 3 \\
\hline PASSBANDS & \\
\hline \# V band & \\
\hline 10.6630 & \\
\hline$\# \mathrm{R}$ band & \\
\hline 20.5887 & \\
\hline \# I band & \\
\hline 30.5090 & \\
\hline$\# \mathrm{H}$ band & \\
\hline 40.3292 & \\
\hline \# Farm Cove, unfiltere & \\
\hline 50.5413 & \\
\hline \# Auck, unfiltered & \\
\hline 60.5490 & \\
\hline OBSERVATIONS & \\
\hline 06 ob-109-auck & \\
\hline $06 o b-109-f c o v$ & \\
\hline $06 o b-109-1 i v$ & \\
\hline $06 \mathrm{ob}-109-\mathrm{mdm}$ & \\
\hline 06ob-109-mfun & \\
\hline 06ob-109-moa & \\
\hline 06ob-109-mtlem & \\
\hline 06ob-109-ogle & \\
\hline $060 \mathrm{o}-109$-tas & \\
\hline 060 -109-wise & \\
\hline
\end{tabular}

Figure F.4: Control file describing a better model for OGLE-2006-BLG-109 and used to generate the lightcurves in Figures 6.7 and 6.8 . 


\section{Bibliography}

Abramowitz, M. and Stegun, I. (1965). Handbook of Mathematical Functions. Applied mathematics series. Dover Publications.

Bachelet, E., Shin, I.-G., Han, C., Fouqué, P., Gould, A., Menzies, J. W., Beaulieu, J.-P., Bennett, D. P., Bond, I. A., Dong, S., Heyrovský, D., Marquette, J.-B., Marshall, J., Skowron, J., Street, R. A., Sumi, T., Udalski, A., Abe, L., Agabi, K., Albrow, M. D., Allen, W., Bertin, E., Bos, M., Bramich, D. M., Chavez, J., Christie, G. W., Cole, A. A., Crouzet, N., Dieters, S., Dominik, M., Drummond, J., Greenhill, J., Guillot, T., Henderson, C. B., Hessman, F. V., Horne, K., Hundertmark, M., Johnson, J. A., Jørgensen, U. G., Kandori, R., Liebig, C., Mékarnia, D., McCormick, J., Moorhouse, D., Nagayama, T., Nataf, D., Natusch, T., Nishiyama, S., Rivet, J.-P., Sahu, K. C., Shvartzvald, Y., Thornley, G., Tomczak, A. R., Tsapras, Y., Yee, J. C., Batista, V., Bennett, C. S., Brillant, S., Caldwell, J. A. R., Cassan, A., Corrales, E., Coutures, C., Dominis Prester, D., Donatowicz, J., Kubas, D., Martin, R., Williams, A., Zub, M., The PLANET Collaboration, de Almeida, L. A., DePoy, D. L., Gaudi, B. S., Hung, L.-W., Jablonski, F., Kaspi, S., Klein, N., Lee, C.-U., Lee, Y., Koo, J.-R., Maoz, D., Muñoz, J. A., Pogge, R. W., Polishook, D., Shporer, A., $\mu$ Collaboration, T. F., Abe, F., Botzler, C. S., Chote, P., Freeman, M., Fukui, A., Furusawa, K., Harris, P., Itow, Y., Kobara, S., Ling, C. H., Masuda, K., Matsubara, Y., Miyake, N., Ohmori, K., Ohnishi, K., Rattenbury, N. J., Saito, T., Sullivan, D. J., Suzuki, D., Sweatman, W. L., Tristram, P. J., Wada, K., Yock, P. C. M., The MOA Collaboration, Szymański, M. K., Soszyński, I., Kubiak, M., Poleski, R., Ulaczyk, K., Pietrzyński, G., Wyrzykowski, Ł., The OGLE Collaboration, Kains, N., Snodgrass, C., Steele, 
I. A., The RoboNet Collaboration, Alsubai, K. A., Bozza, V., Browne, P., Burgdorf, M. J., Calchi Novati, S., Dodds, P., Dreizler, S., Finet, F., Gerner, T., Hardis, S., Harpsøe, K., Hinse, T. C., Kerins, E., Mancini, L., Mathiasen, M., Penny, M. T., Proft, S., Rahvar, S., Ricci, D., Scarpetta, G., Schäfer, S., Schönebeck, F., Southworth, J., Surdej, J., Wambsganss, J., and MiNDSTEp Consortium, T. (2012). MOA 2010-BLG-477Lb: Constraining the Mass of a Microlensing Planet from Microlensing Parallax, Orbital Motion, and Detection of Blended Light. The Astrophysical Journal, 754:73.

Batista, V., Gould, A., Dieters, S., Dong, S., Bond, I., Beaulieu, J. P., Maoz, D., Monard, B., Christie, G. W., McCormick, J., Albrow, M. D., Horne, K., Tsapras, Y., Burgdorf, M. J., Calchi Novati, S., Skottfelt, J., Caldwell, J., Kozłowski, S., Kubas, D., Gaudi, B. S., Han, C., Bennett, D. P., An, J., MOA Collaboration, Abe, F., Botzler, C. S., Douchin, D., Freeman, M., Fukui, A., Furusawa, K., Hearnshaw, J. B., Hosaka, S., Itow, Y., Kamiya, K., Kilmartin, P. M., Korpela, A., Lin, W., Ling, C. H., Makita, B. S., Masuda, K., Matsubara, Y., Miyake, N., Muraki, Y., Nagaya, M., Nishimoto, K., Ohnishi, K., Okumura, T., Perrott, Y. C., Rattenbury, N., Saito, T., Sullivan, D. J., Sumi, T., Sweatman, W. L., Tristram, P. J., von Seggern, E., Yock, P. C. M., PLANET Collaboration, Brillant, S., Calitz, J. J., Cassan, A., Cole, A., Cook, K., Coutures, C., Dominis Prester, D., Donatowicz, J., Greenhill, J., Hoffman, M., Jablonski, F., Kane, S. R., Kains, N., Marquette, J.-B., Martin, R., Martioli, E., Meintjes, P., Menzies, J., Pedretti, E., Pollard, K., Sahu, K. C., Vinter, C., Wambsganss, J., Watson, R., Williams, A., Zub, M., FUN Collaboration, Allen, W., Bolt, G., Bos, M., DePoy, D. L., Drummond, J., Eastman, J. D., Gal-Yam, A., Gorbikov, E., Higgins, D., Janczak, J., Kaspi, S., Lee, C.-U., Mallia, F., Maury, A., Monard, L. A. G., Moorhouse, D., Morgan, N., Natusch, T., Ofek, E. O., Park, B.-G., Pogge, R. W., Polishook, D., Santallo, R., Shporer, A., Spector, O., Thornley, G., Yee, J. C., MiNDSTEp Consortium, Bozza, V., Browne, P., Dominik, M., Dreizler, S., Finet, F., Glitrup, M., Grundahl, F., Harpsøe, K., Hessman, F. V., Hinse, T. C., Hundertmark, M., Jørgensen, U. G., Liebig, C., Maier, G., Mancini, L., Mathiasen, M., Rahvar, 
S., Ricci, D., Scarpetta, G., Southworth, J., Surdej, J., Zimmer, F., RoboNet Collaboration, Allan, A., Bramich, D. M., Snodgrass, C., Steele, I. A., and Street, R. A. (2011). MOA-2009-BLG-387Lb: a massive planet orbiting an M dwarf. Astronomy \& Astrophysics, 529:A102.

Beaulieu, J., Bennett, D. P., Fouqué, P., Williams, A., Dominik, M., Jørgensen, U. G., Kubas, D., Cassan, A., Coutures, C., Greenhill, J., Hill, K., Menzies, J., Sackett, P. D., Albrow, M., Brillant, S., Caldwell, J. A. R., Calitz, J. J., Cook, K. H., Corrales, E., Desort, M., Dieters, S., Dominis, D., Donatowicz, J., Hoffman, M., Kane, S., Marquette, J.-B., Martin, R., Meintjes, P., Pollard, K., Sahu, K., Vinter, C., Wambsganss, J., Woller, K., Horne, K., Steele, I., Bramich, D. M., Burgdorf, M., Snodgrass, C., Bode, M., Udalski, A., Szymański, M. K., Kubiak, M., Więckowski, T., Pietrzyński, G., Soszyński, I., Szewczyk, O., Wyrzykowski, Ł., Paczyński, B., Abe, F., Bond, I. A., Britton, T. R., Gilmore, A. C., Hearnshaw, J. B., Itow, Y., Kamiya, K., Kilmartin, P. M., Korpela, A. V., Masuda, K., Matsubara, Y., Motomura, M., Muraki, Y., Nakamura, S., Okada, C., Ohnishi, K., Rattenbury, N. J., Sako, T., Sato, S., Sasaki, M., Sekiguchi, T., Sullivan, D. J., Tristram, P. J., Yock, P. C. M., and Yoshioka, T. (2006). Discovery of a cool planet of 5.5 Earth masses through gravitational microlensing. Nature, 439:437-440.

Bennett, D. P. (2010). An Efficient Method for Modeling High-magnification Planetary Microlensing Events. The Astrophysical Journal, 716:1408-1422.

Bennett, D. P., Anderson, J., Bond, I. A., Udalski, A., and Gould, A. (2006). Identification of the OGLE-2003-BLG-235/MOA-2003-BLG-53 Planetary Host Star. The Astrophysical Journal, 647:L171-L174.

Bennett, D. P., Bond, I. A., Udalski, A., Sumi, T., Abe, F., Fukui, A., Furusawa, K., Hearnshaw, J. B., Holderness, S., Itow, Y., Kamiya, K., Korpela, A. V., Kilmartin, P. M., Lin, W., Ling, C. H., Masuda, K., Matsubara, Y., Miyake, N., Muraki, Y., Nagaya, M., Okumura, T., Ohnishi, K., Perrott, Y. C., Rattenbury, N. J., Sako, T., Saito, T., Sato, S., Skuljan, L., Sullivan, D. J., Sweatman, W. L., Tristram, P. J., Yock, P. C. M., Kubiak, M., Szymański, 
M. K., Pietrzyński, G., Soszyński, I., Szewczyk, O., Wyrzykowski, Ł., Ulaczyk, K., Batista, V., Beaulieu, J. P., Brillant, S., Cassan, A., Fouqué, P., Kervella, P., Kubas, D., and Marquette, J. B. (2008). A Low-Mass Planet with a Possible Sub-Stellar-Mass Host in Microlensing Event MOA-2007-BLG-192. The Astrophysical Journal, 684:663-683.

Bennett, D. P., Rhie, S. H., Nikolaev, S., Gaudi, B. S., Udalski, A., Gould, A., Christie, G. W., Maoz, D., Dong, S., McCormick, J., Szymański, M. K., Tristram, P. J., Macintosh, B., Cook, K. H., Kubiak, M., Pietrzyński, G., Soszyński, I., Szewczyk, O., Ulaczyk, K., Wyrzykowski, Ł., OGLE Collaboration, DePoy, D. L., Han, C., Kaspi, S., Lee, C.-U., Mallia, F., Natusch, T., Park, B.-G., Pogge, R. W., Polishook, D., $\mu$ FUN Collaboration, Abe, F., Bond, I. A., Botzler, C. S., Fukui, A., Hearnshaw, J. B., Itow, Y., Kamiya, K., Korpela, A. V., Kilmartin, P. M., Lin, W., Ling, J., Masuda, K., Matsubara, Y., Motomura, M., Muraki, Y., Nakamura, S., Okumura, T., Ohnishi, K., Perrott, Y. C., Rattenbury, N. J., Sako, T., Saito, T., Sato, S., Skuljan, L., Sullivan, D. J., Sumi, T., Sweatman, W. L., Yock, P. C. M., MOA Collaboration, Albrow, M., Allan, A., Beaulieu, J.-P., Bramich, D. M., Burgdorf, M. J., Coutures, C., Dominik, M., Dieters, S., Fouqué, P., Greenhill, J., Horne, K., Snodgrass, C., Steele, I., Tsapras, Y., PLANET, F. t., RoboNet Collaborations, Chaboyer, B., Crocker, A., and Frank, S. (2010). Masses and Orbital Constraints for the OGLE-2006-BLG-109Lb,c Jupiter/Saturn Analog Planetary System. The Astrophysical Journal, 713:837-855.

Bennett, D. P., Sumi, T., Bond, I. A., Kamiya, K., Abe, F., Botzler, C. S., Fukui, A., Furusawa, K., Itow, Y., Korpela, A. V., Kilmartin, P. M., Ling, C. H., Masuda, K., Matsubara, Y., Miyake, N., Muraki, Y., Ohnishi, K., Rattenbury, N. J., Saito, T., Sullivan, D. J., Suzuki, D., Sweatman, W. L., Tristram, P. J., Wada, K., and Yock, P. C. M. (2012). Planetary and Other Short Binary Microlensing Events from the MOA Short Event Analysis. arXiv/1203.4560.

Bond, I. A., Udalski, A., Jaroszyński, M., Rattenbury, N. J., Paczyński, B., Soszyński, I., Wyrzykowski, L., Szymański, M. K., Kubiak, M., Szewczyk, O., 
Żebruń, K., Pietrzyński, G., Abe, F., Bennett, D. P., Eguchi, S., Furuta, Y., Hearnshaw, J. B., Kamiya, K., Kilmartin, P. M., Kurata, Y., Masuda, K., Matsubara, Y., Muraki, Y., Noda, S., Okajima, K., Sako, T., Sekiguchi, T., Sullivan, D. J., Sumi, T., Tristram, P. J., Yanagisawa, T., Yock, P. C. M., and OGLE Collaboration (2004). OGLE 2003-BLG-235/MOA 2003-BLG-53: A Planetary Microlensing Event. The Astrophysical Journal, 606:L155-L158.

Cavendish, H., Maxwell, J., Larmor, J., and Thorpe, T. (1921). The Scientific Papers of the Honourable Henry Cavendish. The University Press.

Chang, K. and Refsdal, S. (1979). Flux variations of QSO 0957+561 A, B and image splitting by stars near the light path. Nature, 282:561-564.

Chote, P. (2011). A Semi-Analytical Model for Gravitational Microlensing. Victoria University of Wellington.

Christie, G. (2006). Detecting Exoplanets by Gravitational Microlensing using a Small Telescope. Society for Astronomical Sciences Annual Symposium, 25:97.

Chwolson, O. (1924). Über eine mögliche Form fiktiver Doppelsterne. Astronomische Nachrichten, 221:329.

de Laplace, P. (1799). Exposition du système du monde. Impr. de Crapelet.

Dong, S., Gould, A., Udalski, A., Anderson, J., Christie, G. W., Gaudi, B. S., OGLE Collaboration, Jaroszyński, M., Kubiak, M., Szymański, M. K., Pietrzyński, G., Soszyński, I., Szewczyk, O., Ulaczyk, K., Wyrzykowski, Ł., $\mu$ FUN Collaboration, DePoy, D. L., Fox, D. B., Gal-Yam, A., Han, C., Lépine, S., McCormick, J., Ofek, E., Park, B.-G., Pogge, R. W., MOA Collaboration, Abe, F., Bennett, D. P., Bond, I. A., Britton, T. R., Gilmore, A. C., Hearnshaw, J. B., Itow, Y., Kamiya, K., Kilmartin, P. M., Korpela, A., Masuda, K., Matsubara, Y., Motomura, M., Muraki, Y., Nakamura, S., Ohnishi, K., Okada, C., Rattenbury, N., Saito, T., Sako, T., Sasaki, M., Sullivan, D., Sumi, T., Tristram, P. J., Yanagisawa, T., Yock, P. C. M., Yoshoika, T., PLANET/RoboNet Collaborations, Albrow, M. D., Beaulieu, J. P., Brillant, S., Calitz, H., Cassan, A., Cook, K. H., Coutures, C., Dieters, S., Prester, D. D., Donatowicz, J., 
Fouqué, P., Greenhill, J., Hill, K., Hoffman, M., Horne, K., Jørgensen, U. G., Kane, S., Kubas, D., Marquette, J. B., Martin, R., Meintjes, P., Menzies, J., Pollard, K. R., Sahu, K. C., Vinter, C., Wambsganss, J., Williams, A., Bode, M., Bramich, D. M., Burgdorf, M., Snodgrass, C., Steele, I., Doublier, V., and Foellmi, C. (2009). OGLE-2005-BLG-071Lb, the Most Massive M Dwarf Planetary Companion? The Astrophysical Journal, 695:970-987.

du Sautoy, M. (2009). Symmetry: A Journey into the Patterns of Nature. HarperCollins.

Einstein, A. (1936). Lens-Like Action of a Star by the Deviation of Light in the Gravitational Field. Science, 84:506-507.

Erdl, H. and Schneider, P. (1993). Classification of the multiple deflection two point-mass gravitational lens models and application of catastrophe theory in lensing. Astronomy \& Astrophysics, 268:453-471.

Faà di Bruno, F. (1855). Sullo sviluppo delle Funzioni. Annali di Scienze Matematiche e Fisiche, 6:479480.

Gaudi, B. S., Bennett, D. P., Udalski, A., Gould, A., Christie, G. W., Maoz, D., Dong, S., McCormick, J., Szymański, M. K., Tristram, P. J., Nikolaev, S., Paczyński, B., Kubiak, M., Pietrzyński, G., Soszyński, I., Szewczyk, O., Ulaczyk, K., Wyrzykowski, Ł., OGLE Collaboration, DePoy, D. L., Han, C., Kaspi, S., Lee, C.-U., Mallia, F., Natusch, T., Pogge, R. W., Park, B.-G., $\mu$-Fun Collabortion, Abe, F., Bond, I. A., Botzler, C. S., Fukui, A., Hearnshaw, J. B., Itow, Y., Kamiya, K., Korpela, A. V., Kilmartin, P. M., Lin, W., Masuda, K., Matsubara, Y., Motomura, M., Muraki, Y., Nakamura, S., Okumura, T., Ohnishi, K., Rattenbury, N. J., Sako, T., Saito, T., Sato, S., Skuljan, L., Sullivan, D. J., Sumi, T., Sweatman, W. L., Yock, P. C. M., MOA Collaboration, Albrow, M. D., Allan, A., Beaulieu, J.-P., Burgdorf, M. J., Cook, K. H., Coutures, C., Dominik, M., Dieters, S., Fouqué, P., Greenhill, J., Horne, K., Steele, I., Tsapras, Y., Planet Collaboration, RoboNet Collaborations, Chaboyer, B., Crocker, A., Frank, S., and Macintosh, B. (2008). Dis- 
covery of a Jupiter/Saturn Analog with Gravitational Microlensing. Science, 319:927-.

Gaudi, B. S., Naber, R. M., and Sackett, P. D. (1998). Microlensing by Multiple Planets in High-Magnification Events. The Astrophysical Journal, 502:L33.

Gaudi, B. S. and Petters, A. O. (2002). Gravitational Microlensing near Caustics. II. Cusps. The Astrophysical Journal, 580:468-489.

Gould, A. (2000). A Natural Formalism for Microlensing. The Astrophysical Journal, 542:785-788.

Gould, A. (2008). Hexadecapole Approximation in Planetary Microlensing. The Astrophysical Journal, 681:1593-1598.

Gould, A. and Gaucherel, C. (1997). Stokes's Theorem Applied to Microlensing of Finite Sources. The Astrophysical Journal, 477:580.

Gould, A., Udalski, A., An, D., Bennett, D. P., Zhou, A.-Y., Dong, S., Rattenbury, N. J., Gaudi, B. S., Yock, P. C. M., Bond, I. A., Christie, G. W., Horne, K., Anderson, J., Stanek, K. Z., DePoy, D. L., Han, C., McCormick, J., Park, B.-G., Pogge, R. W., Poindexter, S. D., Soszyński, I., Szymański, M. K., Kubiak, M., Pietrzyński, G., Szewczyk, O., Wyrzykowski, Ł., Ulaczyk, K., Paczyński, B., Bramich, D. M., Snodgrass, C., Steele, I. A., Burgdorf, M. J., Bode, M. F., Botzler, C. S., Mao, S., and Swaving, S. C. (2006). Microlens OGLE-2005-BLG-169 Implies That Cool Neptune-like Planets Are Common. The Astrophysical Journal, 644:L37-L40.

Han, C. (2005). Analysis of Microlensing Light Curves Induced by MultiplePlanet Systems. The Astrophysical Journal, 629:1102-1109.

Han, C. (2006). Properties of Planetary Caustics in Gravitational Microlensing. The Astrophysical Journal, 638:1080-1085.

Hawking, S. and Israel, W. (1989). Three Hundred Years of Gravitation. Philosophiae Naturalis, Principia Mathematica. Cambridge University Press. 
Jaki, S. L. (1978). Johann Georg von Soldner and the gravitational bending of light, with an English translation of his essay on it published in 1801. Foundations of Physics, 8:927-950.

Janczak, J., Fukui, A., Dong, S., Monard, L. A. G., Kozłowski, S., Gould, A., Beaulieu, J. P., Kubas, D., Marquette, J. B., Sumi, T., Bond, I. A., Bennett, D. P., Abe, F., Furusawa, K., Hearnshaw, J. B., Hosaka, S., Itow, Y., Kamiya, K., Korpela, A. V., Kilmartin, P. M., Lin, W., Ling, C. H., Makita, S., Masuda, K., Matsubara, Y., Miyake, N., Muraki, Y., Nagaya, M., Nagayama, T., Nishimoto, K., Ohnishi, K., Perrott, Y. C., Rattenbury, N. J., Sako, T., Saito, T., Skuljan, L., Sullivan, D. J., Sweatman, W. L., Tristram, P. J., Yock, P. C. M., MOA Collaboration, An, J. H., Christie, G. W., Chung, S.-J., DePoy, D. L., Gaudi, B. S., Han, C., Lee, C.-U., Mallia, F., Natusch, T., Park, B.-G., Pogge, R. W., $\mu$ FUN Collaboration, Anguita, T., Calchi Novati, S., Dominik, M., Jørgensen, U. G., Masi, G., Mathiasen, M., MiNDSTEp Collaboration, Batista, V., Brillant, S., Cassan, A., Cole, A., Corrales, E., Coutures, C., Dieters, S., Fouqué, P., Greenhill, J., and PLANET Collaboration (2010). Sub-Saturn Planet MOA-2008-BLG-310Lb: Likely to be in the Galactic Bulge. The Astrophysical Journal, 711:731-743.

Jenkins, M. A. and Traub, J. F. (1970). A three-stage algorithm for real polynomials using quadratic iteration. SIAM Journal on Numerical Analysis, $7(4): 545-566$.

Jenkins, M. A. and Traub, J. F. (1972). Algorithm 419: zeros of a complex polynomial [c2]. Communications of the ACM, 15(2):97-99.

Korpela, A. (2007). Modelling Complex Gravitational Microlensing Events. Victoria University of Wellington.

Mao, S. and Paczyński, B. (1991). Gravitational microlensing by double stars and planetary systems. The Astrophysical Journal, 374:L37-L40.

Marat, J. (1780). Découvertes de M. Marat sur la lumière. Jombert. 
Mekwi, W. (2001). Iterative Methods for Roots of Polynomials. Oxford University.

Miyake, N., Sumi, T., Dong, S., Street, R., Mancini, L., Gould, A., Bennett, D. P., Tsapras, Y., Yee, J. C., Albrow, M. D., Bond, I. A., Fouqué, P., Browne, P., Han, C., Snodgrass, C., Finet, F., Furusawa, K., Harpsøe, K., Allen, W., Hundertmark, M., Freeman, M., Suzuki, D., Abe, F., Botzler, C. S., Douchin, D., Fukui, A., Hayashi, F., Hearnshaw, J. B., Hosaka, S., Itow, Y., Kamiya, K., Kilmartin, P. M., Korpela, A., Lin, W., Ling, C. H., Makita, S., Masuda, K., Matsubara, Y., Muraki, Y., Nagayama, T., Nishimoto, K., Ohnishi, K., Perrott, Y. C., Rattenbury, N., Saito, T., Skuljan, L., Sullivan, D. J., Sweatman, W. L., Tristram, P. J., Wada, K., Yock, P. C. M., MOA Collaboration, Bolt, G., Bos, M., Christie, G. W., DePoy, D. L., Drummond, J., Gal-Yam, A., Gaudi, B. S., Gorbikov, E., Higgins, D., Hwang, K.-H., Janczak, J., Kaspi, S., Lee, C.-U., Koo, J.-R., Kozłowski, S., Lee, Y., Mallia, F., Maury, A., Maoz, D., McCormick, J., Monard, L. A. G., Moorhouse, D., Muñoz, J. A., Natusch, T., Ofek, E. O., Pogge, R. W., Polishook, D., Santallo, R., Shporer, A., Spector, O., Thornley, G., $\mu$ FUN Collaboration, Allan, A., Bramich, D. M., Horne, K., Kains, N., Steele, I., RoboNet Collaboration, Bozza, V., Burgdorf, M. J., Calchi Novati, S., Dominik, M., Dreizler, S., Glitrup, M., Hessman, F. V., Hinse, T. C., Jørgensen, U. G., Liebig, C., Maier, G., Mathiasen, M., Rahvar, S., Ricci, D., Scarpetta, G., Skottfelt, J., Southworth, J., Surdej, J., Wambsganss, J., Zimmer, F., MiNDSTEp Consortium, Batista, V., Beaulieu, J. P., Brillant, S., Cassan, A., Cole, A., Corrales, E., Coutures, C., Dieters, S., Greenhill, J., Kubas, D., Menzies, J., and PLANET Collaboration (2011). A Sub-Saturn Mass Planet, MOA-2009-BLG-319Lb. The Astrophysical Journal, 728:120.

Mollerach, S. and Roulet, E. (2002). Gravitational Lensing and Microlensing. World Scientific.

Muraki, Y., Han, C., Bennett, D. P., Suzuki, D., Monard, L. A. G., Street, R., Jorgensen, U. G., Kundurthy, P., Skowron, J., Becker, A. C., Albrow, M. D., Fouqué, P., Heyrovský, D., Barry, R. K., Beaulieu, J.-P., Wellnitz, D. D., 
Bond, I. A., Sumi, T., Dong, S., Gaudi, B. S., Bramich, D. M., Dominik, M., Abe, F., Botzler, C. S., Freeman, M., Fukui, A., Furusawa, K., Hayashi, F., Hearnshaw, J. B., Hosaka, S., Itow, Y., Kamiya, K., Korpela, A. V., Kilmartin, P. M., Lin, W., Ling, C. H., Makita, S., Masuda, K., Matsubara, Y., Miyake, N., Nishimoto, K., Ohnishi, K., Perrott, Y. C., Rattenbury, N. J., Saito, T., Skuljan, L., Sullivan, D. J., Sweatman, W. L., Tristram, P. J., Wada, K., Yock, P. C. M., MOA Collaboration, Christie, G. W., DePoy, D. L., Gorbikov, E., Gould, A., Kaspi, S., Lee, C.-U., Mallia, F., Maoz, D., McCormick, J., Moorhouse, D., Natusch, T., Park, B.-G., Pogge, R. W., Polishook, D., Shporer, A., Thornley, G., Yee, J. C., $\mu$ FUN Collaboration, Allan, A., Browne, P., Horne, K., Kains, N., Snodgrass, C., Steele, I., Tsapras, Y., RoboNet Collaboration, Batista, V., Bennett, C. S., Brillant, S., Caldwell, J. A. R., Cassan, A., Cole, A., Corrales, R., Coutures, C., Dieters, S., Dominis Prester, D., Donatowicz, J., Greenhill, J., Kubas, D., Marquette, J.-B., Martin, R., Menzies, J., Sahu, K. C., Waldman, I., Williams, A., Zub, M., PLANET Collaboration, Bourhrous, H., Matsuoka, Y., Nagayama, T., Oi, N., Randriamanakoto, Z., IRSF Observers, Bozza, V., Burgdorf, M. J., Calchi Novati, S., Dreizler, S., Finet, F., Glitrup, M., Harpsøe, K., Hinse, T. C., Hundertmark, M., Liebig, C., Maier, G., Mancini, L., Mathiasen, M., Rahvar, S., Ricci, D., Scarpetta, G., Skottfelt, J., Surdej, J., Southworth, J., Wambsganss, J., Zimmer, F., MiNDSTEp Consortium, Udalski, A., Poleski, R., Wyrzykowski, Ł., Ulaczyk, K., Szymański, M. K., Kubiak, M., Pietrzyński, G., Soszyński, I., and OGLE Collaboration (2011). Discovery and Mass Measurements of a Cold, 10 Earth Mass Planet and Its Host Star. The Astrophysical Journal, 741:22.

Newton, I. (1718). Opticks: or, A treatise of the reflections, refractions, inflections, and colours of light. Printed for W. and J. Innys.

Paczyński, B. (1986). Gravitational microlensing by the galactic halo. The Astrophysical Journal, 304:1-5.

Press, W. H., Teukolsky, S. A., Vetterling, W. T., and Flannery, B. P. (1992). 
Numerical recipes in $C$ (2nd ed.): the art of scientific computing. Cambridge University Press, New York, NY, USA.

Rattenbury, N. J., Bond, I. A., Skuljan, J., and Yock, P. C. M. (2002). Planetary microlensing at high magnification. Monthly Notices of the Royal Astronomical Society, 335:159-169.

Rhie, S. H. (2001). Can A Gravitational Quadruple Lens Produce 17 images? arXiv:astro-ph/0103463.

Rhie, S. H. (2002). How Cumbersome is a Tenth Order Polynomial?: The Case of Gravitational Triple Lens Equation. arXiv:astro-ph/0202294.

Soldner, J. (1801). Über die ablenkung eines lichtstrahls von seiner geradlinigen bewegung durch die attraktion eines weltkörpers, an welchem er nahe vorbeigeht. Berliner Astronomisches Jahrbuch, 1804:161-172.

Sumi, T., Bennett, D. P., Bond, I. A., Udalski, A., Batista, V., Dominik, M., Fouqué, P., Kubas, D., Gould, A., Macintosh, B., Cook, K., Dong, S., Skuljan, L., Cassan, A., Abe, F., Botzler, C. S., Fukui, A., Furusawa, K., Hearnshaw, J. B., Itow, Y., Kamiya, K., Kilmartin, P. M., Korpela, A., Lin, W., Ling, C. H., Masuda, K., Matsubara, Y., Miyake, N., Muraki, Y., Nagaya, M., Nagayama, T., Ohnishi, K., Okumura, T., Perrott, Y. C., Rattenbury, N., Saito, T., Sako, T., Sullivan, D. J., Sweatman, W. L., Tristram, P. J., Yock, P. C. M., MOA Collaboration, Beaulieu, J. P., Cole, A., Coutures, C., Duran, M. F., Greenhill, J., Jablonski, F., Marboeuf, U., Martioli, E., Pedretti, E., Pejcha, O., Rojo, P., Albrow, M. D., Brillant, S., Bode, M., Bramich, D. M., Burgdorf, M. J., Caldwell, J. A. R., Calitz, H., Corrales, E., Dieters, S., Dominis Prester, D., Donatowicz, J., Hill, K., Hoffman, M., Horne, K., Jørgensen, U. G., Kains, N., Kane, S., Marquette, J. B., Martin, R., Meintjes, P., Menzies, J., Pollard, K. R., Sahu, K. C., Snodgrass, C., Steele, I., Street, R., Tsapras, Y., Wambsganss, J., Williams, A., Zub, M., PLANET Collaboration, Szymański, M. K., Kubiak, M., Pietrzyński, G., Soszyński, I., Szewczyk, O., Wyrzykowski, Ł., Ulaczyk, K., OGLE Collaboration, Allen, W., Christie, G. W., DePoy, D. L., 
Gaudi, B. S., Han, C., Janczak, J., Lee, C.-U., McCormick, J., Mallia, F., Monard, B., Natusch, T., Park, B.-G., Pogge, R. W., Santallo, R., and $\mu$ FUN Collaboration (2010). A Cold Neptune-Mass Planet OGLE-2007-BLG-368Lb: Cold Neptunes Are Common. The Astrophysical Journal, 710:1641-1653.

Sumi, T., Kamiya, K., Bennett, D. P., Bond, I. A., Abe, F., Botzler, C. S., Fukui, A., Furusawa, K., Hearnshaw, J. B., Itow, Y., Kilmartin, P. M., Korpela, A., Lin, W., Ling, C. H., Masuda, K., Matsubara, Y., Miyake, N., Motomura, M., Muraki, Y., Nagaya, M., Nakamura, S., Ohnishi, K., Okumura, T., Perrott, Y. C., Rattenbury, N., Saito, T., Sako, T., Sullivan, D. J., Sweatman, W. L., Tristram, P. J., Udalski, A., Szymański, M. K., Kubiak, M., Pietrzyński, G., Poleski, R., Soszyński, I., Wyrzykowski, Ł., Ulaczyk, K., and Microlensing Observations in Astrophysics (MOA) Collaboration (2011). Unbound or distant planetary mass population detected by gravitational microlensing. Nature, 473:349-352.

Tisserand, P., Le Guillou, L., Afonso, C., Albert, J. N., Andersen, J., Ansari, R., Aubourg, É., Bareyre, P., Beaulieu, J. P., Charlot, X., Coutures, C., Ferlet, R., Fouqué, P., Glicenstein, J. F., Goldman, B., Gould, A., Graff, D., Gros, M., Haissinski, J., Hamadache, C., de Kat, J., Lasserre, T., Lesquoy, É., Loup, C., Magneville, C., Marquette, J. B., Maurice, É., Maury, A., Milsztajn, A., Moniez, M., Palanque-Delabrouille, N., Perdereau, O., Rahal, Y. R., Rich, J., Spiro, M., Vidal-Madjar, A., Vigroux, L., Zylberajch, S., and EROS-2 Collaboration (2007). Limits on the Macho content of the Galactic Halo from the EROS-2 Survey of the Magellanic Clouds. Astronomy \& Astrophysics, 469:387-404.

Treder, H. J. and Jackisch, G. (1981). On Soldners Value of Newtonian Deflection of Light. Astronomische Nachrichten, 302:275.

Tsapras, Y., Street, R., Horne, K., Snodgrass, C., Dominik, M., Allan, A., Steele, I., Bramich, D. M., Saunders, E. S., Rattenbury, N., Mottram, C., Fraser, S., Clay, N., Burgdorf, M., Bode, M., Lister, T. A., Hawkins, E., Beaulieu, J. P., Fouqué, P., Albrow, M., Menzies, J., Cassan, A., and Dominis-Prester, 
D. (2009). RoboNet-II: Follow-up observations of microlensing events with a robotic network of telescopes. Astronomische Nachrichten, 330:4.

Udalski, A., Jaroszyński, M., Paczyński, B., Kubiak, M., Szymański, M. K., Soszyński, I., Pietrzyński, G., Ulaczyk, K., Szewczyk, O., Wyrzykowski, Ł., Christie, G. W., DePoy, D. L., Dong, S., Gal-Yam, A., Gaudi, B. S., Gould, A., Han, C., Lépine, S., McCormick, J., Park, B.-G., Pogge, R. W., Bennett, D. P., Bond, I. A., Muraki, Y., Tristram, P. J., Yock, P. C. M., Beaulieu, J.-P., Bramich, D. M., Dieters, S. W., Greenhill, J., Hill, K., Horne, K., and Kubas, D. (2005). A Jovian-Mass Planet in Microlensing Event OGLE-2005-BLG-071. The Astrophysical Journal, 628:L109-L112.

Walsh, D., Carswell, R. F., and Weymann, R. J. (1979). 0957 + 561 A, B - Twin quasistellar objects or gravitational lens. Nature, 279:381-384.

Wambsganss, J. (1997). Discovering Galactic planets by gravitational microlensing: magnification patterns and light curves. Monthly Notices of the Royal Astronomical Society, 284:172-188.

Wilkinson, J. (1965). The Algebraic Eigenvalue Problem. Clarendon Press, Oxford.

Witt, H. J. (1990). Investigation of high amplification events in light curves of gravitationally lensed quasars. Astronomy $\&$ Astrophysics, 236:311-322.

Yee, J. C., Shvartzvald, Y., Gal-Yam, A., Bond, I. A., Udalski, A., Kozlowski, S., Han, C., Gould, A., Skowron, J., Suzuki, D., the MOA Collaboration, the OGLE Collaboration, and the MicroFUN Collaboration (2012). MOA2011-BLG-293Lb: A test of pure survey microlensing planet detections. The Astrophysical Journal.

Zwicky, F. (1937). Nebulae as Gravitational Lenses. Physical Review, 51:290-290. 